\title{
SELF MANAGEMENT STRATEGIES FOR PEOPLE WITH SICKLE CELL DISEASE IN GHANA
}

BY

ANDREWS ADJEI DRUYE

\begin{abstract}
A thesis
submitted to the Victoria University of Wellington

in fulfilment of the requirements for the degree of

Doctor of Philosophy in Nursing
\end{abstract}

Victoria University of Wellington 


\begin{abstract}
Sickle cell disease (SCD) is the commonest inherited haemoglobinopathy worldwide. Although it is a chronic disease, there is presently no international best practice for selfmanagement of SCD. This exploratory sequential mix-methods study was designed to describe the self-management strategies utilised and their effectiveness for persons with SCD in Ghana. Self-management was conceptualised as actions that concerned preventive health, self-monitoring, self-diagnosing, self-treatment and self-evaluation. A four-stage sequential data collection and analysis process was used. Methods included website review, key informant interviews and surveys. The website review extracted from publically available websites the advice provided to people with SCD regarding keeping well, minimising the development of complications and managing complications. Health professional $(n=9)$ interviews concerned self-management advice and the experiences patients shared of their self-management. Patients with SCD (n=9) and parents of children with SCD (n=10) interviews captured their experiences of self-management; and 112 patients ( $\geq 16$ years) and 201 parents of children ( $\leq 15$ years) were surveyed about their self-management strategies. Analysis used descriptive and inferential statistics and content and thematic techniques. Patients and parents reported that despite receiving limited clinical advice on selfmanagement they undertook at least one self-management action daily. Most patients reported using multiple strategies; preventive health and maintenance actions were the commonest. Whilst most actions were directed at pain, patients and parents also outlined strategies for managing problems such as fever, leg ulcers, anaemia, priapism, abdominal swelling, jaundice, and hip pain. Most self-management actions were clinically safe, however several unsafe actions identified could pose health risks to patients. Overall, parents tended to be more frequent users of self-management actions for their children than the adults were for themselves. Apart from being an adult or child, self-management was not significantly influenced by key vulnerabilities and contextual factors. Although, many patients and parents had tried traditional practices, most only utilised recommendations from health professionals. Most patients and parents had rated the quality their or their child's quality of life as good. There is a need for the development of an international, evidenced-informed approach to selfmanagement of SCD. The Ministry of Health, health professionals and the Sickle Cell
\end{abstract}


Association of Ghana could work collectively on this to ensure a culturally appropriate and economically viable approach is developed and implemented.

\section{Keywords}

Ghana, SCD, Self-Management, Strategies, Quality of life, mixed method, sequential design 


\section{Acknowledgements}

I wish to acknowledge all who enabled and supported me on my journey to complete this $\mathrm{PhD}$. This journey commenced five years ago when I submitted an application for a Victoria University doctoral scholarship which has been essential in enabling me to study at Victoria. I am also appreciative of my employers - University of Cape Coast - who released me to complete this journey. I look forward to returning to share with others what I have learnt along the way. Thank you also to the Faculty of Humanities and Social Sciences, Victoria University which awarded me a grant to support my travel back to Ghana for data collection.

I would like to especially thank all the adults, young people and parents of children with sickle cell disease (SCD) who were interviewed and who completed the survey. I have learnt a lot from your personal insights into how you manage your own or your children's SCD. I know others will benefit from the analysis of your combined wisdom. Thank you also to the health professionals who provided expert advice on SCD self-care in Ghana and who participated in the interviews. I say AYEKOOOO. Thank you also to the health organisations of Ghana - Ghana Health Service, Komfo Anokye Teaching Hospital, Bekwai Government Hospital, Mampong Government Hospital, Effiduasi Government Hospital and , the Sickle Cell Association of Ghana - that supported my access to the research participants. And to my colleagues in Ghana who assisted me with recruitment when issues became difficult because of Ebola, many thanks to you.

To my supervisors Dr Kathy Nelson and Dr Brian Robinson thanks for your guidance and support professionally and personally. I have enjoyed the supervision meetings with you and have learned from your knowledge, expertise, critiques, advice and humour. Brian, the warm Kathmandu jacket you purchased for me has been an essential item of clothing while I have been here in New Zealand. Kathy I have valued your friendship and advice and I look forward to returning the hospitality when you visit me in Ghana. Thanks also to other staff at the Graduate School for your generosity and assistance.

I also wish to acknowledge my friends and fellow students especially Michelle, Restuning, Jenny and Sushil whom I shared an office with. I wish each of you well on your $\mathrm{PhD}$ journey and future academic careers. 
To my lovely wife Gifty, my heartfelt thanks for your love and care during the study. I would never have been able to achieve this without you and am so happy that we have shared this experience. To my little Kiwis Xaria and Kaelan - you are our links with New Zealand forever, and I hope as adults that you will one day get to revisit the country where you were born. Thank you for ensuring I had fun times daily and for your laughter and smiles when things were tough, it helped put this thesis in perspective. To my parents, I am indebted to you for your prayers and words of encouragement anytime you called.

Finally, I couldn't have done anything without you Almighty God. May your name be praised forever for your continual sustenance. 


\section{TABLE OF CONTENTS}

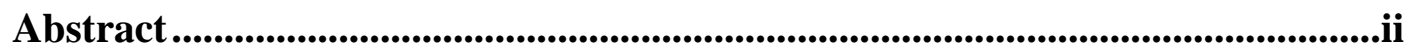

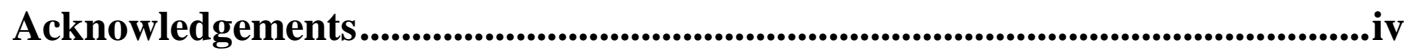

List of Figures ....................................................................................................................xiv

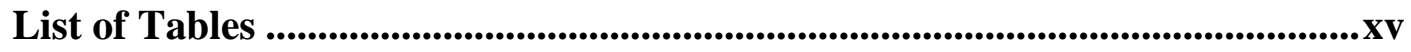

Chapter 1: Introduction to the Research ......................................................1

1.1 General Overview and Concepts of Self-Management..................................2

1.1.1 The roles and responsibilities of patients in self-management.....................

1.1.2 The role of health professionals in self-management ..................................5

1.1.3 The benefits of self-management …...................................................

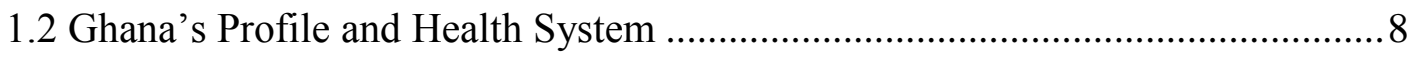

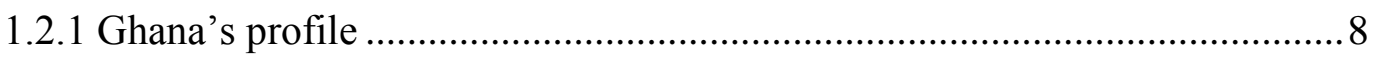

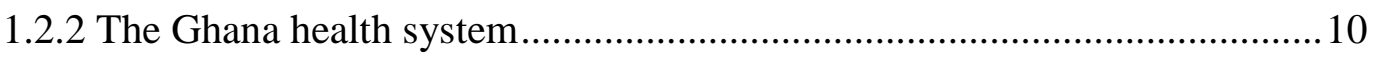

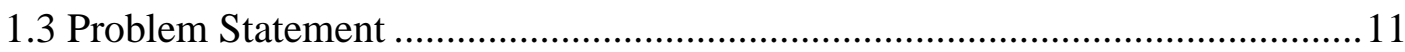

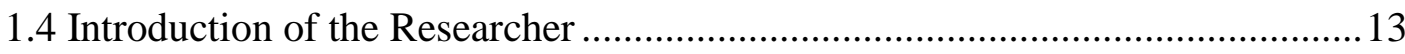

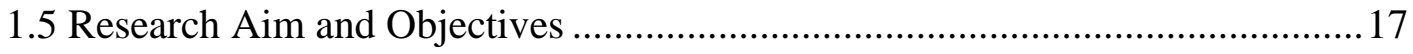

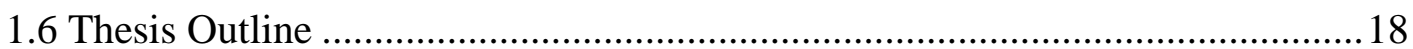

Chapter 2: Overview of SCD and Management in Ghana................................20

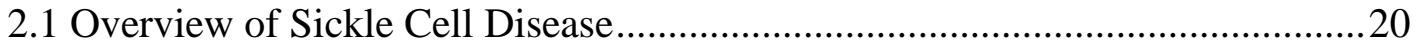

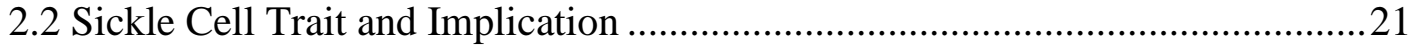

2.3 Aetiology and Epidemiology of Sickle Cell Disease .......................................23

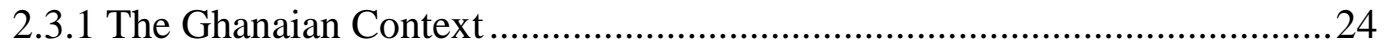

2.4 Pathophysiology and Clinical Features ........................................................24 
2.4.1 Natural History and Clinical Trajectory and Prognosis .26

2.5 Psychosocial Burden of Sickle Cell Disease .29

2.6 Treatment and Management of SCD .30

2.6.1 Health maintenance .31

2.6.2 Treatment of complications 32

2.6.3 The role of the nurse in SCD management .33

2.6.4 Patients' and families' responsibilities in SCD management...... .35

2.7 SCD Management in the Ghanaian Health System 37

Chapter 3: Literature Review 43

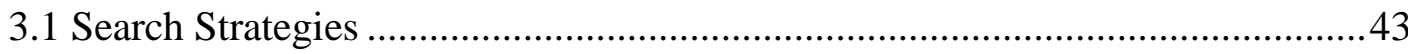

3.2 Overview Self-Management Studies on Genetic Diseases ...............................44

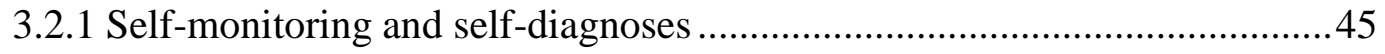

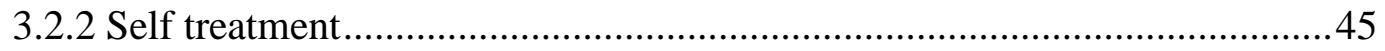

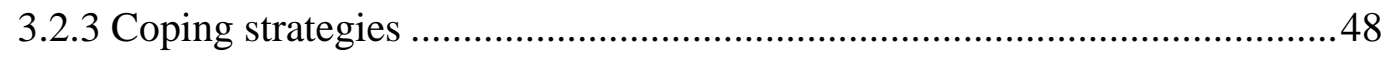

3.3 Research on Self-Management for Sickle Cell Disease ...................................49

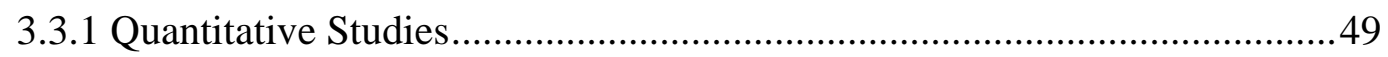

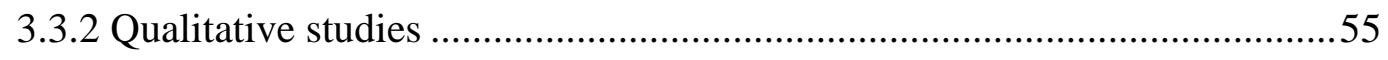

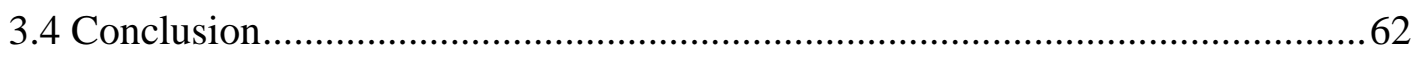

Chapter 4: Conceptual Framework .............................................................665

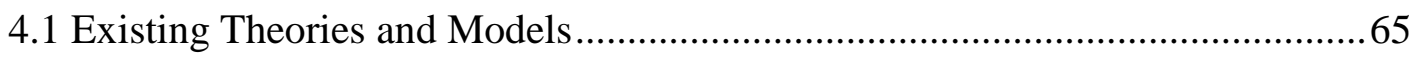

4.1.1 Theories of individual and family self-management ..............................65

4.1.2 The theory of self-care management for vulnerable populations ................67 


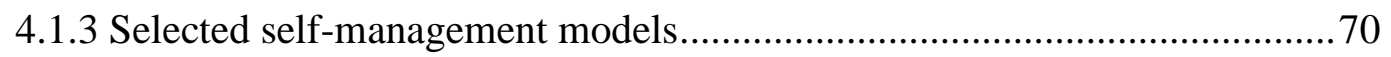

4.2 Application of Modules and Concept to Current Study .................................... 72

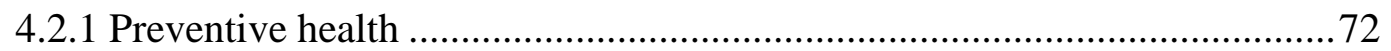

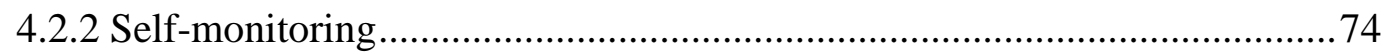

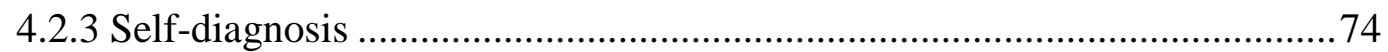

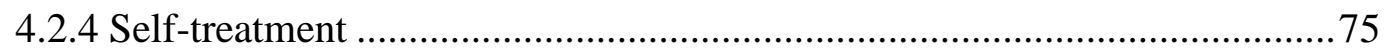

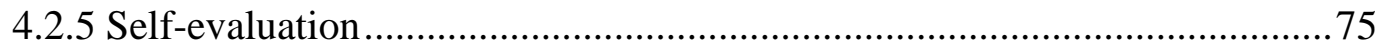

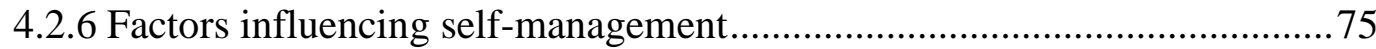

4.2.7 Outcome

Chapter 5: Methodology .....................................................................................78

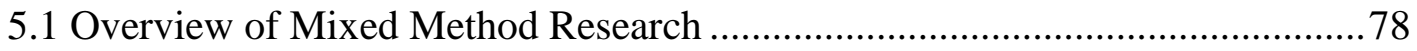

5.1.1 The philosophical worldview of the study ............................................ 78

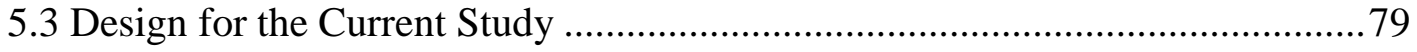

5.4 Overview of Phases, Stages and Methods for the Study ................................... 81

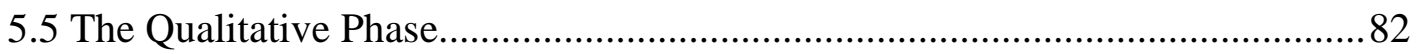

5.5.1 Overview and rationale for using qualitative content analysis ................... 82

5.5.2 Stage 1: Review of SCD focused websites............................................. 85

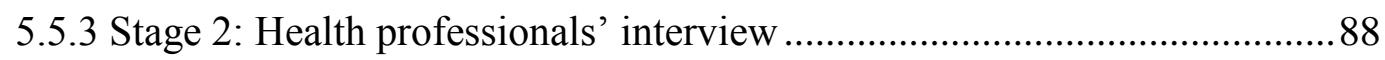

5.5.3 Stage 3: Patients and parents interviews.............................................. 91

5.5.4 Procedure for maintaining trustworthiness of interviews .......................... 93

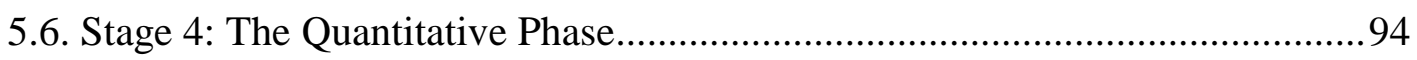

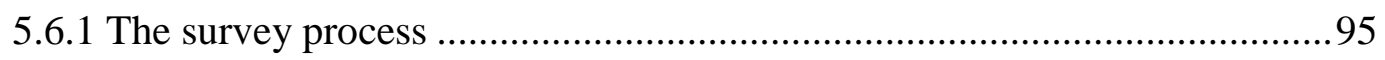


5.6.2 Instrument development

5.6.3 Conducting the survey .98

5.6.4 Data analysis 100

5.7 Ethical Considerations. 102

Chapter 6: Results of Website Review 105

6.1 Overview of Websites, Sources and Characteristics 105

6.2 Self-Management Recommendations 107

6.3 Self-management Actions 108

6.3.1 Preventive Health (Quantitative Results) 108

6.3.2 Self-monitoring 119

6.3.3 Self-Diagnosis

6.3.4 Self-treatment 122

6.4 Self-Management Determinants and Strategies

6.4.1 Managing Psychosocial Challenges ................................................... 126

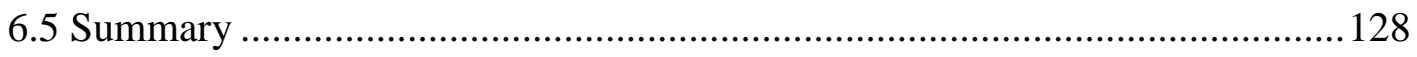

Chapter 7: Perspectives of Health Professionals .............................................129

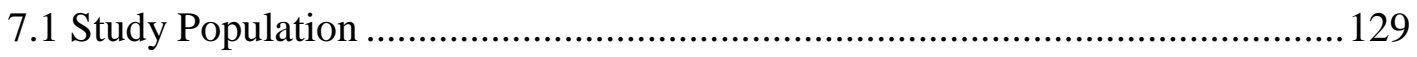

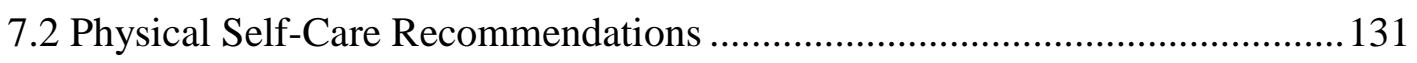

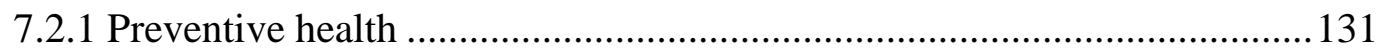

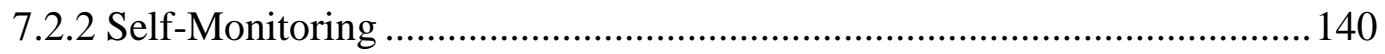

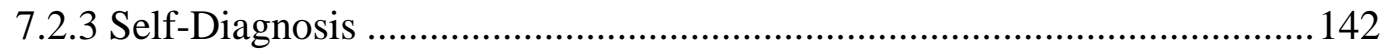

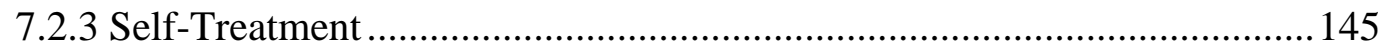




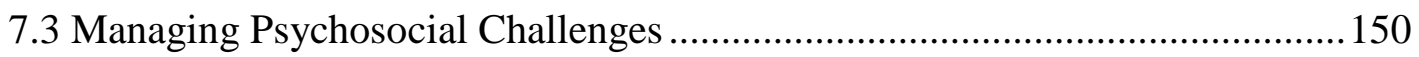

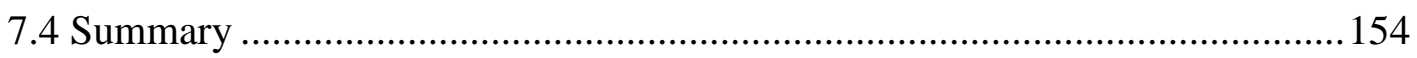

Chapter 8: Perspectives of Patients and Parents .............................................156

8.1 Socio-demographic Characteristics of the Participants.................................... 156

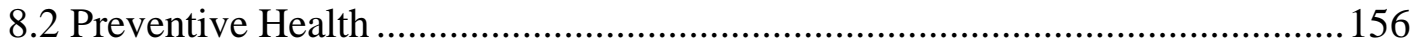

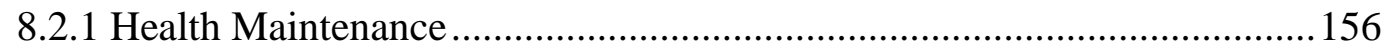

8.2.3 Consultations with Health Professionals ................................................. 161

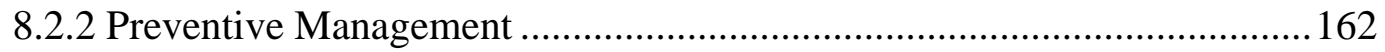

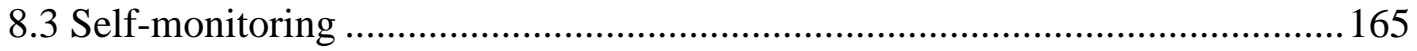

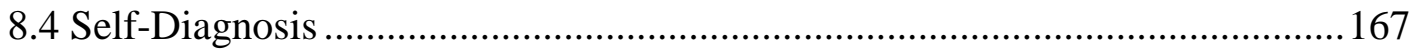

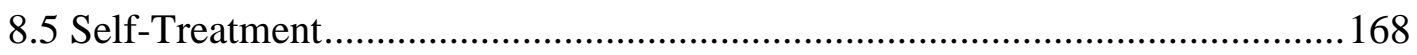

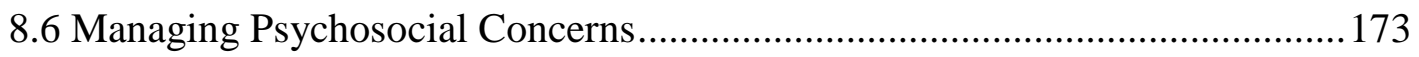

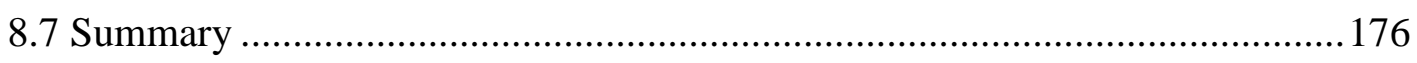

Chapter 9: Quantitative Survey ................................................................................178

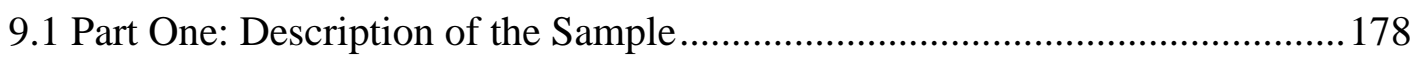

9.1.1 Socio-Demographic Characteristics of the Sample ................................ 179

9.2 Major Problems Patients experienced in the Past 12 months.......................... 181

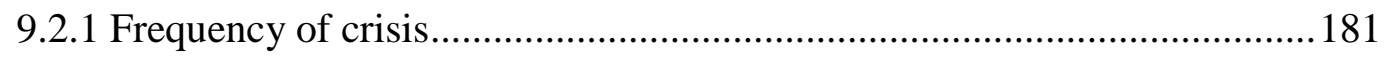

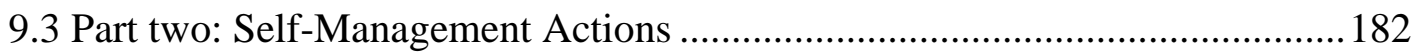

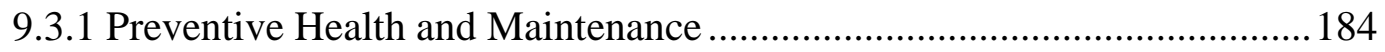

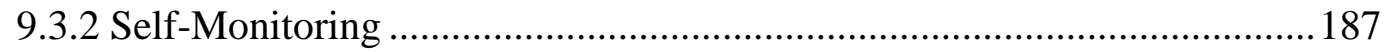

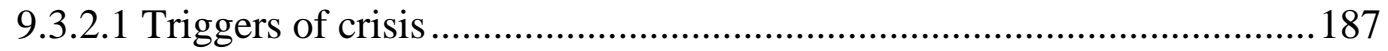

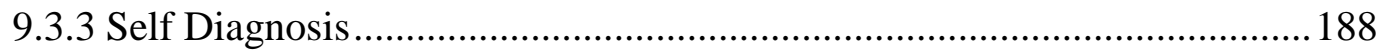


9.3.5 Frequency of selected self-treatment actions. 195

9.4 Prevalence of Conventional and Traditional Medicines Used 198

9.5 Part three: Evaluation of Self-Treatment Actions 200

9.6 Part four: Health Status and Quality of Life 200

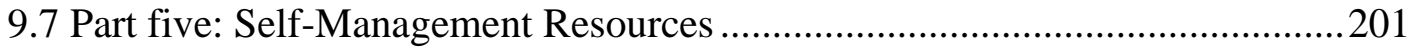

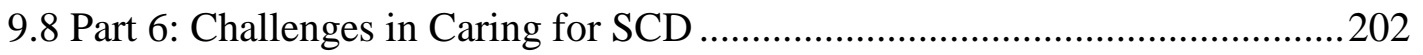

Chapter 10: Discussion and Conclusion..........................................................204

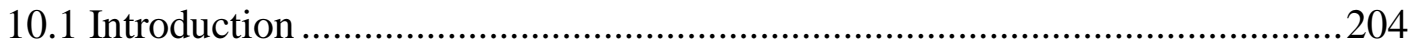

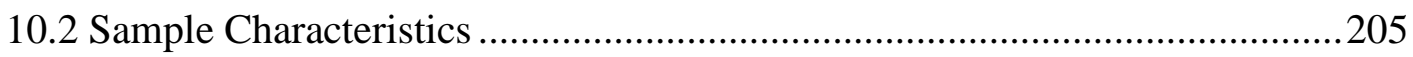

10.3 Key Self-Management Recommendations and Practices .......................207

10.3.1 Recommendations from Websites and Health Professionals .................207

10.3.2 Summary of Patients' and Parents' Practices .........................................209

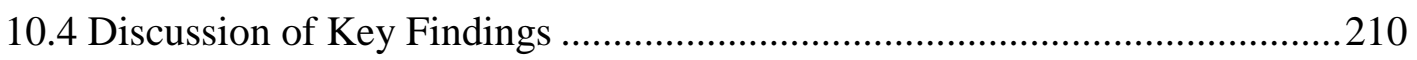

10.4.1 Key Self-Management Practices ....................................................210

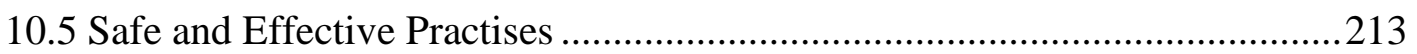

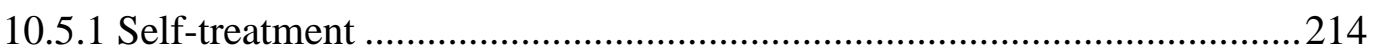

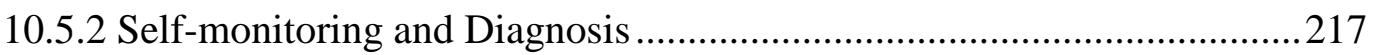

10.5.3 Preventive and Maintenance Actions ..................................................217

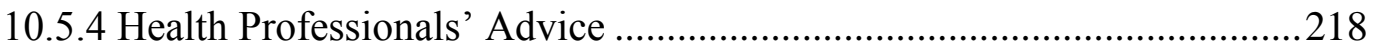

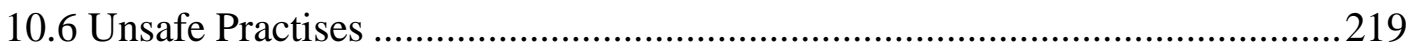

10.6.1 Misuse of Prescribed and Over-the-Counter Medicines........................219 
10.7.1 Reflection on the conceptual framework .226

10.8 Limitations of the Study. .228

10.9 Implications and Recommendations .230

10.9.1 Implications for International and Local Policy 230

10.9.2 Recommendations for SCD Association 231

10.9.3 Recommendations for Nurses and Other Clinicians 231

10.10 Statement of Conclusion and Contribution 236

Appendices 238

Appendix 1. Victoria University of Wellington Ethics Approval 238

Appendix 2. Ghana Health Service Ethics Review Committee Approval. 239

Appendix 3. Permission from Ashanti Regional Health Directorate 240

Appendix 4. Komfo Anokye Teaching Hospital Approval 241

Appendix 5. Komfo Anokye Teaching Hospital Approval 2. 242

Appendix 6. Approval from Komfo Anokye Teaching Hospital 243

Appendix 7. Komfo Anokye Teaching Hospital Supervisor's Support Letter 244

Appendix 8. Sickle Cell Association of Ghana, Approval Letter 245

Appendix 9. KATH Information Leaflet. 246

Appendix 10. Parental Consent Forms for Children Under 18 Years 251

Appendix 11. GHS Consent Above 18 years 253

Appendix 12. Health Professional's Interview Guide. .255 


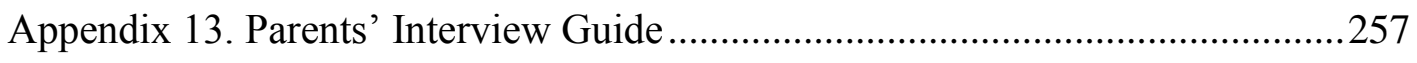

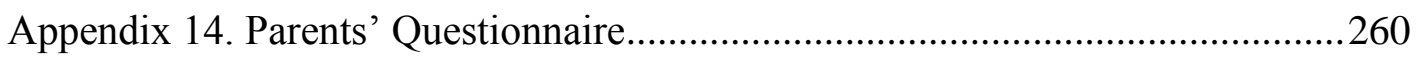

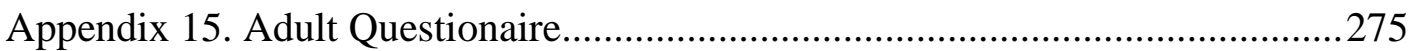

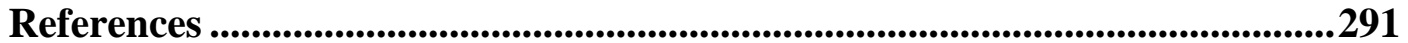




\section{List of Figures}

Figure 1. Map of Ghana showing the ten administrative regions ...................................................... 9

Figure 2. Conceptual framework for self-management strategies for sickle cell disease in Ghana ...... 76

Figure 3. Phases and stages of data collection, analysis and interpretation ........................................ 81

Figure 4. Revised conceptual framework for SCD. 


\section{List of Tables}

Table 1. Common forms of SCD, characteristics and geographical distribution.................................... 22

Table 2. Annual Global Predicted Estimates of Hb AS and Hb SS among Neonates........................... 24

Table 3. Age related Manifestations of SCD in Ghana ...................................................................... 28

Table 4. Ten Competencies of the Nurse in managing SCD ................................................................... 34

Table 5. Summary of variables and concepts derived from literature review ...................................... 63

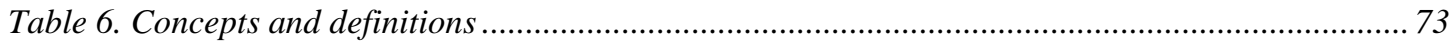

Table 7. Publications used to inform the methods of the current study................................................. 84

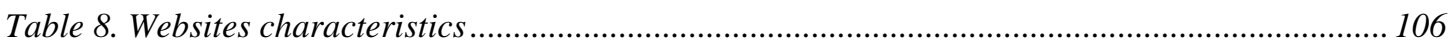

Table 9. Distribution of materials and types by country of website ............................................... 107

Table 10. Target audience and authors' background of materials by website .................................... 107

Table 11. References to self-management actions and determinants ................................................ 108

Table 12. References to preventive health behaviour....................................................................... 109

Table 13. References to self-monitoring of disease by websites........................................................ 120

Table 14. References to self-diagnosis by website....................................................................... 121

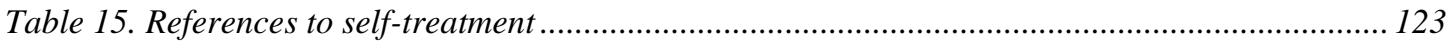

Table 16. References to self-management determinants by website ................................................... 126

Table 17. Characteristics of the health professional participants................................................... 130

Table 18. Socio-demographic characteristics of parents/guardians and children.............................. 157

Table 19. Socio-demographic characteristics of adult participant .................................................... 157

Table 20. Socio-demographic characteristics of respondents........................................................ 180

Table 21. Socio-demographic characteristics of adults and children groups .................................... 181

Table 22. Problems patients have experienced in the last 12 months ................................................ 182

Table 23. Frequency of crises patients have experienced in the past 12 months ............................. 182

Table 24. Summary self-management actions by participants .......................................................... 182

Table 25. Comparison of self-management actions by participant's characteristics......................... 183

Table 26. Summary of frequency of preventive health actions ....................................................... 185

Table 27. frequency of preventive health actions ........................................................................... 186

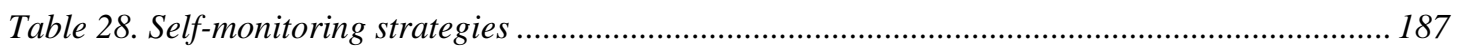

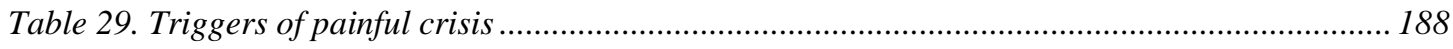

Table 30. Indicators for recognising painful crisis ........................................................................... 189

Table 31. Indicators for recognising non-crisis problems .......................................................... 189

Table 32. Summary of scd problem requiring self treatment action................................................ 190

Table 33. Self-treatment actions for painful episodes ........................................................................ 191

Table 34. Self-treatment action for fever, jaundice, and anaemia ................................................. 192

Table 35. Self-treatment action for headaches, weakness, ulcers and abdominal swelling ................ 193

Table 36. Frequency of use of selected self-treatment actions by regular and periodic use ................ 196 
Table 37. Frequency of use selected self-treatment actions by adult and child.....

Table 38. Use of conventional medicines

Table 39. Use of traditional medicine …..................................................................................... 199

Table 40. Respondent's evaluation of self-treatment actions for individual conditions........................ 200

Table 41. Self-rated health status and quality of life ................................................................... 201

Table 42. Self-management resources............................................................................................. 202

Table 43. Challenges associated with caring for SCD ..................................................................... 203 


\section{Chapter 1: Introduction to the Research}

Sickle Cell Disease (SCD) is a serious genetic disorder of the blood exerting adverse physical, psychological and socio-economic consequences on many patients and families worldwide. This haemoglobin disorder is characterised by severe pains, chronic anaemia, increased risk for infections and multiple organ complications which can significantly impact on the health status and quality of life of those born with SCD. The disease is life-long with a limited opportunity for cure. Providing resources to treat affected people remains a major challenge to nations with low financial resources, including Ghana [1-4]. People living with long term conditions (LTCs) such as SCD require health service intervention to manage acute and on-going complications, supportive care for health maintenance, and psychosocial care. Access to a holistic combination of these interventions results in improvements in health outcomes and quality of life (QOL) of patients [5-7]. The interventions required by people with LTCs transcend the health delivery system to include homes and communities [7]. These interventions may be managed directly by health professionals or by patients and families through self-management.

In recent years there have been a global movement for the involvement of patients with LTCs in their care. It is argued that patients living with LTCs perform self-management; irrespective of whether they have been trained or not $[8,9]$. To this end, Lorig and Holmes [10] argue that "unless one is totally ignorant of healthful behaviours it is impossible not to manage ones health (p. 1)." Patients and carers undertake $90 \%$ of the tasks related to daily living with LTCs whilst health professionals perform the remaining 10\% [11]. Whilst patients and carers spend minutes with their health professionals, they spend their remaining time managing symptoms, medications and the incapacity resulting from the condition [8]. Thus patients (and families) with LTCs are responsible for the management of symptoms, treatment and responding to the physical and psychosocial impact of their diseases on a daily basis through self-management $[10,12]$. It is now generally accepted that patients can actively engage in their care through self-management $[10,13]$. Increased participative patient centred care through self-management leads to greater confidence and better satisfaction with their health [13].

Over the past four decades, self-management of LTCs has received increased attention by many countries. The attention is more common in countries such as the United Kingdom 
(UK), United States of America (USA), Australia, Canada, Japan, New Zealand and China where NCDs constitute a greater proportion of disease burden. In these countries dedicated programmes have been incorporated into the public health systems [13, 14]. Stimuli such as increasing occurrence of LTCs, rising costs of health care, the predominant focus of health systems on acute illnesses, the threat of economic burden of LTCs on a country's physical resources, and the awareness of the patient as an important health resource have driven the focus on self-management $[8,15]$. Consequently, it is believed that self-management will be the principal means of managing LTCs for the future [8]. SCD is a LTC and lends itself to self-management, hence pursuing endeavour in self-management for this condition as this study seeks to achieve is relevant. The knowledge and technology for health-professional-led management for SCD is well established internationally [16]. However, there is a paucity of established standards for self-management in current literature.

This thesis reports on research designed to understand the role of self-management strategies in reducing complications and improving the quality of life of persons living with CSD in Ghana. The study contributes to scholarly efforts on self-management which is increasingly promoted as a cost effective option for improving the health status and quality of life of people with LTCs.

The remainder of this chapter provides an introduction to self-management, a brief overview of Ghana's profile in terms of geography, population characteristics, and sociocultural context relevant to the study including highlighting the region of the study and the rationale for choosing this region. An overview of the health system of Ghana is also presented. This is followed by the problem statement, the introduction of the researcher, and the aims and objectives of this study. The final section presents the structure of the thesis.

\subsection{General Overview and Concepts of Self-Management}

Self-management is a relatively new concept in health care delivery and its definition and understanding continues to evolve. The term was first used in asthma management in the mid-1960s [10]. In general usage, self-management implies taking responsibility of one's own behaviour and well-being. In relation to health, the term generally connotes a situation when a patient is actively involved in decision making and actual management of their condition [10]. In the research community, several synonyms exist such as self-care [13], self-care 
management [17, 18], self-management preparation, and self-management education [18]. Currently, there are no gold standard definitions [19] but two main perspectives exist depending on the roles and responsibilities of health professionals or patients.

The first perspective views self-management as interventions or treatments delivered by health or other professionals for patients with LTCs. Other terms related to this perspective are self-management preparation, self-management education and self-management support $[9,18,20,21]$. For example, Redman [9] defines self-management as "training that people with chronic health conditions need to be able to deal with taking medicines and maintaining therapeutic regimes, maintaining everyday life such as employment and family, and dealing with the future, including changing life plans and the frustrations, anger, and depression (p. 4)."

The second perspective views self-management in terms of patients' performance of certain actions, tasks or behaviours to live with their LTC [10, 22-24]. Such a perspective views self-management as an outcome of self-interventions or patients' management practices irrespective of interactions with health professionals. Adams, Greiner and Corrigan [25] describe self-management as "relating to the task an individual must undertake to live well with one or more chronic conditions. These tasks include gaining confidence to deal with medical management, role management and emotional management (p. 57)." Similarly Clark et al. [23] view self-management as:

the day-to-day task an individual must undertake to control or reduce the impact of disease on physical health status. At home management task and strategies are undertaken with the collaboration and guidance of individual's physician and other health care providers. Self-management, however, also requires the ability to cope with psychosocial problems generated or exacerbated by chronic disease. (p. 5)

The current study embraces the second perspective as it emphasises patient strategies.

Self-management and self-care are often used synonymously but these terms have similarities and differences. Firstly, both terms concern people managing themselves. The individual is both the agent of the action and the object of the action [15]. Secondly, both concern performance of specific behaviours to achieve outcomes but differ in process and outcomes [19]. Self-care concerns healthy lifestyle behaviours undertaken by individuals, or 
preventive strategies to promote health [22]. Ryan and Sawin [19] maintain that self-care involves daily activities and behaviours without collaborating with health providers. Clark et al. [23] argue that the term self-care may not describe the care provided for LTCs. Selfmanagement, on the other hand, concerns the purposeful performance of specific learned behaviours to deal with chronic disease [19]. This involves the ability of the individual to monitor their disease, and develop and utilise cognitive, behavioural and emotional strategies to improve their quality of life [26]. Contrary to self-care, self-management requires consultation with health professionals $[10,19]$. Self-care concerns the prevention of disease, whereas self-management concerns what patients do, on a daily basis, to control the impact of their existing LTCs. In the current self-management literature, both terms are often used for the same purpose, based on conceptual definition. In this thesis, self-management and selfcare are used interchangeably to describe activities and strategies that patients perform on a daily basis to manage their LTC. Patients and health professionals have different roles and responsibilities in patients' self-management and the next section will examine these roles.

\subsubsection{The roles and responsibilities of patients in self-management}

Self-management has been viewed as a life-long activity performed by persons with LTC [23]. The processes involves has been referred to as tasks, skills, activities or work [10, 23, 26, 27]. Clark et al. [23] classified these processes into two distinct, but interactive tasks: at-home tasks and psychological coping. At home tasks are the activities undertaken to manage the clinical aspect of the disease outside health facilities. Psychosocial coping refers to the mechanisms used to deal with the emotional dimensions of their disease. Following a literature review of five LTCs (heart disease, asthma, chronic pulmonary obstructive disease, arthritis, diabetes), Clark et al. [23] outlined self-management tasks common to these conditions: 1) recognising and responding to symptoms, monitoring physical indicators, controlling triggers, 2) using medicines, 3) managing acute episodes and emergencies, 4) maintaining nutrition and diet, 5) maintaining adequate exercise and activities, 6) giving up smoking, 7) using relaxation and stress reduction techniques, 8) interacting with health care providers, 8) seeking information and using community services, 9) adapting to work, 10) managing relations with significant others, and 11) managing emotions and psychological response to illness. In contrast, Lorig and Holmes [10] summarise self-management tasks into three components: 1) medical management of condition for example, taking medication and 
adhering to prescribed diet, 2) maintaining, changing and creating new meaningful behaviours or life roles such as work behaviour and engaging in sports, and 3) dealing with emotional sequels of living with chronic disease such as anger, frustration and depression.

Patients require skills to perform self-management. Lorig and Holmes [10] describe five core skills patients require to perform self-management tasks effectively. The first involves problem solving including problem definition, generation of solutions, implementation of solutions, and evaluation of results. The second skill is decision-making regarding the day-to-day changes in disease status. The third involves the skills in using education resources such as the internet, library and other community resources. The fourth includes skills in forming partnerships with health providers including accurately reporting disease trends, making informed decisions about treatment, and engaging in effective communication with health professionals. The final skill is taking action. This skill enables patients to learn behaviour change strategies. Planning and implementation of actions are key features of this skill. More recently, Ryan and Sawin [19] referred to self-management as selfregulatory skills involving goal setting, self-monitoring, reflective thinking, decision making, planning and engaging specific behaviours, self-evaluation and the management of physical, emotional and cognitive responses associated with health behaviour change. It can be concluded that self-management is an active process and deliberate effort is required to learn specific skills and behaviours to manage the impact of LTCs.

\subsubsection{The role of health professionals in self-management}

Whilst patients' performances form the foundation of self-management, the role of health professionals is to provide self-management support. As observed [28] "selfmanagement is not isolated from medical care; rather, self-management is aimed at helping the participant become an active, not adversarial, partner with health care providers" (p. 3). Thus health professionals and patients collaborate to promote self-management. It has been theorised that when "informed activated patients" interact with a "prepared, proactive, practice team" the result is improved "functional and clinical outcomes" [7].

Self-management support is defined by Adams et al. [25] "the systematic provision of education and supportive interventions by health care staff to increase patients skills and confidence in managing their health problems, including regular assessment of progress and 
problems, goal setting, and problem-solving support" (p. 57). Alternatively, the Department of Health UK [13] refers to self-management support as comprising policies, self-care information, self-monitoring devices, self-care skills education, training and self-care support networks. These two perspectives highlight the nature of input (education, policies, material resources), process (regular assessment, goal setting, problem solving) and outcomes (patients skills and confidence in managing problems) for self-management. Thus, as observed by the Department of Health UK [13], engagement of health professionals is one of the critical tools for successful application of self-management education programmes.

Self-management support is enhanced by three key factors: knowledge or health literacy, skills and social facilitation for health maintenance and behaviour change [19, 29]. It also involves provision of equipment such as self-care tools, self-monitoring devices, assistive technologies, and promotion of patients involvement in community networks that can offer additional information, tools and devices and other supports [13]. Education or health literacy is important in assisting patients to make appropriate health decisions [29]. Scholars have distinguished between self-management education and traditional health education. Traditional health education provides technical information for specified diseases and focuses on building knowledge with the goal of achieving patient compliance. The expected outcomes are increased patient knowledge, satisfaction and readiness to engage healthy behaviour [19, 29]. Self-management education on the other hand focuses on how to act on problems identified by individual patients. The purpose is to enable patients to manage their condition in ways that are flexible and relevant to their lives. It focuses on building patients' confidence rather than making patients compliant to medical prescriptions as in traditional health education $[10,18,30]$. The expected outcomes of self-management education include the acquisition of skills for behaviour change, decreased health care cost, increased QOL and improved well-being [19]. Self-management education also aims to equip patients and carers with the ability to recognise signs and symptoms, manage the physical and psychological demands of the LTC and ensure early referral for medical attention where necessary [31]. Therefore it can be argued that self-management education traverses traditional patient education to include strategies to build the confidence and skills of patients and caregivers, take leadership and control of their condition and manage themselves in ways suitable to them, rather than adhering to instructions from medical sources. 
Self-management is complex, multidimensional, and context specific [10]. Hence it must be viewed from patient's perceived concerns and problems [10,19]. Consequently, selfmanagement interventions must be patient centred, culturally relevant and involve systematic follow up.

\subsubsection{The benefits of self-management}

Self-management is beneficial to patients, families, carers and the health system. It enables patients with LTCs to live longer, healthier, and independent lives [29]. Selfmanagement interventions result in increased knowledge for patients and carers, enabling them to perform management behaviours to delay disease progression, resulting in improve clinical, physical and psychological outcomes in addition to QOL [8, 24]. It also reduces the disruptiveness of LTCs on family life and improve family functioning [32].

For patients living with LTCs, incorporating self-management in everyday lives results in independence, self-worth, and the ability to lead as near a normal life as possible [13]. The benefits gained by engaging in self-management are improved physical and mental health and wellbeing, reduced perception of the severity of symptoms, improved compliance, a greater sense of confidence and control, reduced need for emergency healthcare, social services including hospital admissions, as well as planned and coordinated care $[8,13]$. The DOH identified the investment in self-management reduces GP visits by approximately $40 \%$, outpatient visits by $17 \%$, and accident and emergency department presentations by $50 \%$ with reduced expenditure drug.

Two internally recognised LTC self-management programmes - The Chronic Conditions Self-Management Programme and the Expert Patient Programme have demonstrated practical benefits of self-management programmes. These programmes aim to empower patients through self-management training. A range of empirical research from the programmes across different medical conditions, cultures and languages has demonstrated that these programmes improve patient outcomes, create rational use of health resources and reduce health care costs [33-36]. No publication was located regarding the application of these multi-component programmes for SCD. However, a proposal for an Expert Patient Programme for SCD was located in the USA [37]. 


\subsection{Ghana's Profile and Health System}

\subsubsection{Ghana's profile}

Ghana is located in West Africa covering a land area of 238,537 square kilometres, sharing borders with La Côte d'Ivoire, Burkina Faso, the Republic of Togo and the Gulf of Guinea [38] (Figure 1). In 2010, the population of Ghana was estimated to be 27 million with a male to female ratio of $95.2: 100$. The population is predominantly youthful; $38.5 \%$ were 15 years or younger and $4.7 \%$ were 65 years or older in 2010 . The population density is 103 per square kilometre with an average household composition of 4.4 occupants. Approximately half of the population live in urban areas [39].

There are 100 tribal groups with unique cultures and languages, commonly grouped into four ethnic groups: Akan (45.5\%), Mole-Dabani (16.6\%), Ewe (13.9\%) and Ga-Dangbe (7.4\%). These groups share a common cultural heritage, language and origin [39, 40]. All regions of Ghana are ethnically mixed especially the urban centres. English is the official language for education and public communication and Akan is the most widely spoken local language [40]. The literacy rate is approximately $74 \%$ of the population 11 years or older. Approximately 67\% can read and write English, 20\% can write English only, and 53\% can write at least one local language. Other common languages are French and Arabic [40]. Religious affiliations are Christianity (71.2\%), Islam (17.6\%) and African traditional religions (5.2\%) [39]. The role of religion is important for the adoption of beliefs and practices influencing health [41]. The country is geographically divided into northern and southern parts and 10 administrative regions (Figure 1). 


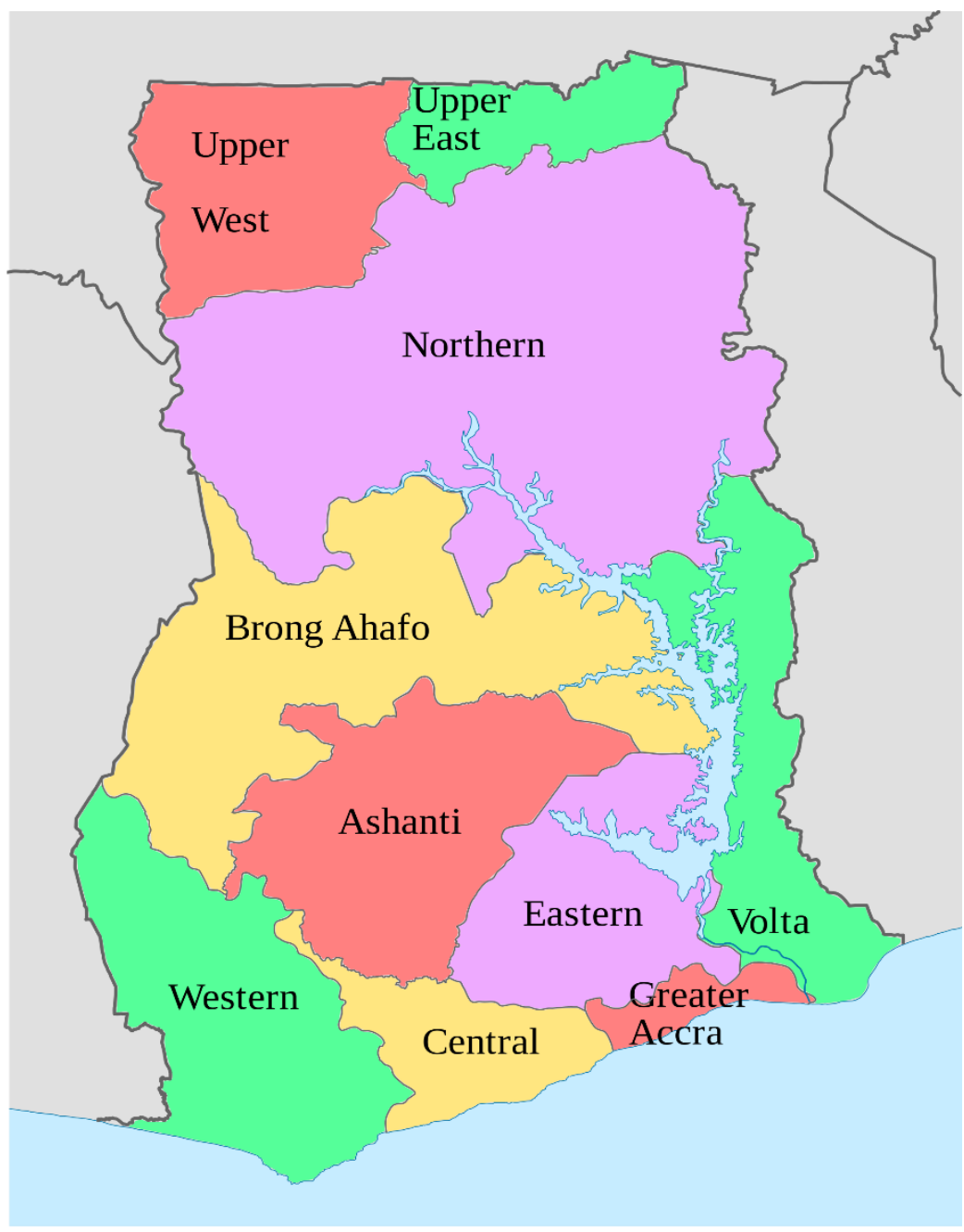

FigURE 1. MAP OF GHANA SHOWING THE TEN ADMINISTRATIVE REGIONS

Source: https://commons.wikimedia.org/wiki/File:Regions_of_Ghana_en.svg

Ghana has a tropical climate with an average temperature of $26^{\circ} \mathrm{C}$. Weather patterns differ geographically; the southern regions have raining seasons from April to June and September to November and the northern regions have one rainy season from May to September [38]. The weather and rainfall patterns have implications for the occurrence of SCD crisis and related problems.

The Ashanti region, where this study was conducted, is located in the south. The region is the most populous accounting for 4,780,380 (19.4\%) of the country's population [39]. Ashanti and the Greater Accra (the national capital) are the regions containing $50 \%$ of the population and are the most urbanised and industrialised [38, 39]. Ashanti is traditionally an Akan community where it is possible to communicate with the majority of the populace 
through the Akan language. The regional capital, Kumasi, which was the major study site, is centrally located with link roads to many parts of the country. The city is characterised by external and internal migration within Ghana and from neighbouring countries making it culturally diverse and characterised by rapid urbanisation [40].

The choice of the Ashanti region for the study was because it was the setting for the Newborn Screening for Sickle Cell Disease Project (NSSCDP). Until 2016, it remained the only region screening new-borns for SCD in the public health system in Ghana. The Komfo Anokye Teaching Hospital (KATH) in Kumasi, the centre for the NSSCD project, has the largest number of SCD patients in Ghana. This hospital receives patients from most of the other surrounding regions [42]. The KATH SCD clinic (urban) and three rural SCD clinics and associations were the settings for sample selection and data collection.

\subsubsection{The Ghana health system}

The Ghana health system comprises public and private institutions under the auspices of the $\mathrm{MOH}$. Health services are delivered through public and private institutions (agencies) on behalf of the Ministry. There were 3110 health delivery facilities in 2008 of which $67.7 \%$ were public facilities. The public sector consists of the Ghana Health Service (GHS), teaching hospitals and regulatory bodies. The GHS is the largest public health agency responsible for the delivery of primary and secondary care services [43]. The GHS was established by the GHS and Teaching Hospitals Act 525/1996, and is responsible for the administration and management of state-owned health facilities excluding teaching hospitals, quasi-state institutions (military and police hospitals) and mission health facilities [44]. The GHS is functionally organised at national, regional, district, sub-district and community levels comprising government-owned hospitals, maternity homes, clinics, health centres, polyclinics [43]. The service is organised into clinical care and public health units. Teaching hospitals have "Centres of Excellence" that receive referrals from Ghana and other countries.

The private sector complements and collaborates with the public sector, providing $32 \%$ of health services through private non-profit and private for-profit providers [44]. Mission-based providers, organised by the Christian Health Association of Ghana, are the largest private non-profit health delivery agents [45]. The Government funds about $80 \%$ of salaries of their staff [44]. Private for-profit providers include private hospitals, clinics, 
maternity homes, pharmacies and chemists forming $26.3 \%$ of health facilities [45]. The private for-profit also includes the traditional sector including practitioners such as traditional birth attendants, herbalists, bone setters, and spiritual or faith healers [46, 47]. The Ministry of Health (2005) is in the process of integrating traditional medicines into the formal health system.

Infectious diseases including malaria, upper respiratory tract infections and diarrhoea and non-communicable diseases including asthma, hypertension, diabetes and SCD are commonly managed at outpatient (OPD) services [45]. Ghanaian health indicators have progressively improved resulting in increased in life expectancy at birth from 44 years in 1960 to 61 years in 2015 [48, 49]. Whilst Ghana records a superior life expectancy compared to most neighbouring West African countries, the figures were below the world average of 71years in 2015 [49]. Furthermore, Ghana's global ranking in life expectancy declined from 117 to 163 from 1960 to 2015 [48]. Total government expenditure on health, as a percentage of GDP, was 5.1\% in 2009, which was also below Africa and global averages of $5.5 \%$ and $8.7 \%$ respectively [45]. SCD is managed at all levels of health care in the Ghana Health System but the services are insufficient to meet the demands for care. Details of SCD management within the health system are described next.

\subsection{Problem Statement}

Ghana has a long history of SCD predating the scientific discovery approximately a century ago [50]. The disease is well known in most communities and Ghanaians ascribe various local names that depict the nature of the pain and complications. Scientific work to understand SCD pathology and epidemiology in Ghana commenced in the 1950s [51, 52]. Recent research, through a new-born screening programme, indicates that 1 in 50 births in Ghana (16,000-18,000 babies per year) are born with SCD [53-55]. In comparison, the disease affects 1 in 500 Black or African-Americans and 1 in 36,000 Hispanics in USA and is recognised as a public health concern [56]. It is worth noting that more children are born with SCD than HIV which is considered a major public health problem in Ghana [57]. In addition $20-25 \%$ of new-born children carry either the sickle cell trait or haemoglobin C trait [54, 55, $58]$. Due to the decreased life expectancy of people with SCD, the population with SCD in Ghana is estimated to be around 400,000 [59]. The Ghanaian health system cannot currently 
provide services comparable to developed nations to manage SCD, and therefore new approaches, such as self-management, are required for improving the QOL of patients and reducing the disease burden on patients, families and national resources.

Official recognition and establishment of organised public health programmes for SCD have only recently been implemented in Ghana. Despite some achievements, there is limited application of the WHO recommended public health measures for SCD management $[4,60]$. The WHO recommendations include advocacy, prevention and counselling, early detection, case management, follow-up, data collection, surveillance and research, community education and partnership [61]. In most parts of Ghana, these services are either absent, unstructured or delivered on an ad hoc basis [4, 62]. Current policy, programmes and services, focus on new-born screening and clinical management or presenting problems. There are few specialised SCD clinics and experts, limited laboratory support and treatment protocols, lack of follow-up care, and limited organised patient-focused education and counselling [4, 62]. Therefore, major service gaps exist in SCD focused services.

Screening and clinical treatment of physical problems are provided but these do not offer holistic care necessary for improving the QOL for SCD patients. Treatment services do not routinely include psychosocial interventions nor interventions that focus on building the personal capacities of patients and families to effectively self-manage the day-to-day burden of the disease. Given that SCD is a LTC characterised by illness patterns that constantly impact on patients' daily lives, affected persons and families are expected to self-manage over $90 \%$ of the day-to-day burden $[8,11]$. It has been argued that as patients with LTCs such as SCD engage in self-management it is unethical to deny training and support to self-manage appropriately [9]. In the absence of organised self-management interventions in Ghana and limited access to SCD focused treatment services, patients are likely to develop their own means to self-manage their disease.

In my earlier employment (2002-2010) as a nurse at SCD clinic and educator for patients and parents, I noticed some patients' self-management treatments through personal observations and patients' reports. Whilst some of the measures appeared harmless, others were potentially dangerous including the use and misuse of traditional medicines and practices and the misuse of orthodox medicines. These observation made me wonder: how 
frequent these observations and reports I received at the clinic are? Do other practices remain unidentified? Are other health professionals aware of these practices? How do patients' selfmanagement actions impact on their health? And, what lessons can be learnt from practices to improve SCD care?

A literature search failed to identify documentation of self-management for SCD in African countries such as Ghana. Moreover, self-management has not been considered in SCD policy or programmes in Ghana. The lack of scientific knowledge of self-management measures in Ghana is a concern because the measures people employ, and the impact on patients' health are unknown. Furthermore, it implies that there is no scientific knowledge that can serve as a basis to influence policy, and inform the development of locally appropriate self-management interventions for SCD. Consequently, as Ghana strives to provide better care and services for SCD patients, empirical knowledge of self-management strategies will be instrumental to understanding what people do and how it impacts on their health. This information will inform policy and programming for self-management interventions, and improved care delivery for SCD in Ghana.

\subsection{Introduction of the Researcher}

I was inspired to pursue this doctoral study due to my affiliation with the NSSCD Project. The NSSCDP was a longitudinal study which screened new-borns for SCD in Kumasi and those diagnosed were enrolled in a specialised SCD clinic for comprehensive management. The project consisted of screening, education and counselling, clinical care and laboratory components. A local branch of the Sickle Cell Association of Ghana (SCAG), comprising patients and parents, was attached to the clinic to provide psychosocial support. The project was financed by the National Institute of Health, USA with Professor Kwaku Ohene-Frempong (a Ghanaian) from the University of Pennsylvania as the principal investigator. The project ran from 1993 until 2008. The MOH proposed a national programme modelled after the NSSCD Project to scale up SCD interventions across Ghana in 2008 due to the success of the project.

I joined the NSSCD project in 2002 as a patient educator and counsellor at the clinic and association. I became the coordinator of the education and counselling component in 2007 after completing my Master's degree and was appointed as the National Focal Person 
for SCD in Ghana by the MOH to lead the scaling up of the project into a national programme. I was also the General-Secretary for the Sickle Cell Association of Ghanaian (SCAG). However, the programme NSCD programme did not progress as planned due to lack of funding. Consequently, I joined academia in 2010 while maintaining my roles with the SCAG and links with the national programme. During my clinic time, engagement with SCAG and role on the programme, I observed a number of undesirable situations and gaps that "angered" me and for which I have been seeking an opportunity to assist in addressing.

At the SCD clinic, I observed that patients were unwilling to attend other health services as they reported receiving inadequate care and preferred to wait for the SCD clinic which was held twice a week. For example, a 41 year-old SCD patient told me that other hospitals were incapable of managing SCD adequately; his condition worsened when he attends those services. Non-attendance was also an issue and clinic staff reported frequent non-attendance for scheduled appointments with many patients attending only when experiencing serious problems. The NSSCD project (2006) reported that approximately $23 \%$ of SCD patients failed to attend the SCD clinic. Stigmatisation was cited as the main reason with long waiting hours at the clinic, transportation difficulties, geographical, financial and health literature challenges commonly cited for non-attendance. Even children who were diagnosed with SCD through the new-born screening programme failed to attend clinics. Consequently, I wondered whether non-attending patients understood the importance of clinic attendance and also how these patients manage their SCD.

At the clinics, I had observed that many first attendants (parents) had limited understanding of their own sickling status. Data I collected between August and December, 2007 (unpublished) to evaluate the education and counselling services showed that $97 \%$ of parents were unaware of their sickling status prior to having a child with SCD. The proportion of mothers who were unaware of their genotype decreased to $80 \%$ after their first clinic appointment but that of fathers remained unchanged. The issues regarding limited knowledge of SCD in the Ghanaian population has been raised previously by the SCAG and SCD advocates including myself [63]. However, until now no large scale study has been conducted to describe the knowledge of SCD among the population of Ghana. A study by Dennis Antwi and colleagues found knowledge lapses among health workers in Kumasi prior to commencement of the NSSCDP [64]. 
Another observation I made at the clinic was limited paternal involvement in caring for SCD children. Although fathers' perspectives were partially addressed in a $\mathrm{PhD}$ thesis by Dennis-Antwi (2006) investigating the socio-cultural factors that influence fathers' perception of SCD children, the consequences of fathers' involvement in the care and outcome on the health of children remain unresolved. Given that fathers constitute the main income earners for most families in Ghana, limited paternal involvement shifts responsibilities to mothers. As most mothers are likely to be unemployed or in low income employment, as I observed at the SCAG, limited paternal involvement sometimes resulted in inadequate resources to provide care for health or general needs of the affected child. This may adversely affect the quality of life of the child, and adversely affect the mother psychologically.

I also personally observed many activities and issues at the Association held monthly sessions where I was a coordinator and an educator. Activities included continuous education, discussion forums, entertainments and distribution of essential medicines (paracetamol and folic acid). Many parents (mostly mothers) complained of financial challenges that impacted on medical, transportation and general care. Many were unable to afford the premium of the National Health Insurance Scheme costing between GH\$7.20 to GH\$48.00 annually in 2008. Consequently, the SCAG periodically raised funds to assist parents to register with the Scheme. It was common practice for project staff, including myself, to support patients and parents financially with their medication, transportation or general care. Having health insurance did not guarantee health care as some health facilities were not accredited with the National Health Insurance Scheme. Moreover, some accredited facilities refused to attend to patients with insurance due to delay in receiving reimbursement from the National Health Insurance Scheme.

At the SCAG forums, parents frequently expressed difficulties in having their children take medications or engage in other preventive measures. Many lamented that their SCD children were stubborn, while others confessed frustrations as a result of frequent sickness, hospitalisation, financial loss and other SCD care challenges. Some expressed the wish that their children were dead.

I also noted that some patients self-administer opioid analgesics especially morphine and pethidine. Such patients use various strategies to obtain the medicines. For example a 39 
year old man attempted to trick me to write a prescription for morphine for him as his prescription was "lost". This same patient, and a few others, had tried similar tricks on other nurses and doctors. Some had learnt to request prescriptions and solicit funds from unsuspecting sympathisers to acquire these medicines. My question then was: why would an SCD patient with access to a clinic abuse morphine, and how common was the abuse of morphine and other prescription drugs? Patients also mentioned having managed some complications including priapism, ulcers, and hand foot-syndrome at home. The specific strategies, frequency and safety of the measures the patients used were unknown.

Another area of observation at both clinic and association concerned the use of traditional medicine. Some patients reported use of traditional medicines at the forums. For example, a 60 year old patient shared his experience of using dried pawpaw leaves, administered through an enema to manage his crises. It is common practice in Ghana to observe traditional healers in various media platforms professing cures for LTCs including SCD, diabetes, hypertension, and cancers. I observed that patients informed by health professionals about the incurable nature of SCD, were convince by traditional healers that they could be cured. Traditional healers were frequently sighted selling products to patients outside the clinic. Related to traditional medicine practice was the issue of spirituality. Patients tended to recount their experiences of either being blamed as witches or their disease being caused by witches. Some reported having engaged in prayers, visiting shrines and other traditional practices for spiritual healing. The question then arose as to the nature of traditional medicines people use, the prevalence, reasons for use, sources, mode of administration, and characteristics of patients that patronise these medicines. I also wondered about the helpfulness, dangers, and lessons that can be learnt from users to inform our advice to other SCD patients.

Some observations were also made during my role as the National SCD Focal Person in Ghana. By 2013, only 4 of the 10 regions had SCD clinics in their regional hospitals but only two (Korle-bu, Komfo Anokye) functioned consistently. All the clinics were located in a regions capital (Kumasi, Accra/Tema, Koforidua, Sunyani). Apart from the Korle-bu clinic that provides out-patient services from Monday to Friday, the others operate on a weekly or monthly basis. Therefore, many patients, especially those in rural areas had limited access due to distance, cost of transportation, and knowledge about the existence of SCD clinics. 
Consequently little is known about how such patients typically manage their disease. I also observed that the central focus of Ghana's national SCD proposed programme is bio-medical management. With an emphasis on new-born screening, clinical care, and genetic testing and counselling and testing. Limited focus was given to home and community-based care.

To sum up, my experience with SCD management in Ghana showed several lapses that has the potential to adversely affect patients' health outcomes. These include default and non-attendance for clinical appointments, patients unwillingness to use other health resources, limited knowledge of SCD and related care among patients, challenges with following medical advice, self-treatment of disease and complications, use of traditional medicines and local remedies, and over-emphasis on clinical treatment. In addition, many patients were faced with challenges with access to SCD clinics due to overcrowding, long waiting hours, long distance, transportation challenges and fear of stigmatisation. Many of the observations described above could have adverse physical and psychological implication on patients' health and need to be addressed. In particular I observed that many patients had selfmanagement measures they adopt for their disease. However the extent, types, source, and impact of these measures on patients' health were unknown. Scanning the literature, I identified that patients with LTCs tend to engage in self-management and proper training in self-management can improve patients' physical and psychological health, and QOL. However, self-management is contextually based, hence, understanding what people do in a given context will help to inform the development of an effective self-management programme. This study was therefore, undertaken to understand self-management strategy for SCD in Ghana. It was anticipated that the study would elicit patients' strategies for purposes of scientific documentation, programme development and improved care.

\subsection{Research Aim and Objectives}

The aim of the research is to understand the role of self-management strategies in reducing complications and improving the quality of life of persons living with SCD in Ghana. The objectives are to:

- Establish self-management advice provided in SCD focused websites and by SCD experts in Ghana;

- Identify the measures patients and families take to prevent illness and reduce complications; 
- Determine the strategies patients and families use in monitoring their disease;

- Determine the strategies patients and families use to recognise SCD related problems and complications;

- Determine the specific self-management actions taken by patients in response to recognised problems and complications; and

- Elicit the major factors underpinning self-management practices among patients and families.

\subsection{Thesis Outline}

This thesis consists of 10 chapters set out in three parts. Part one (introduction, overview of SCD and management in the Ghana Health System, literature review, and conceptual framework) provides general background information and positions the study within the context of previous and current scholarly efforts in SCD self-management. This introductory chapter has presented an overview of Ghana's profile and health system, the problem statement, an introduction to the researcher, and the aims and objectives of the study. The second chapter presents an overview of SCD and the management of SCD within the Ghana Health System. The chapter also highlights the nature of the disease, historical and epidemiological context, current practices and technologies for management. It further analyses the current SCD management within the Ghana Health System compared with international recommendations of best practice. In Chapter 3, I present and critique the previous research on SCD self-management and identify the gaps that informed the research question. In Chapter 4, I combine evidence from previous research, relevant self-management theories and models, and my personal experiences to develop a conceptual framework to guard the study.

Part two focuses on the research strategies used to answer the research question. Reflecting on gaps in literature and theories, and lessons drawn from how research in selfmanagement has been conducted, I outline the methodology in Chapter 5. The chapter focuses on describing the philosophical underpinning of the choice of methodology (mixed method), design issues, procedures and instruments for data gathering, data analysis and presentation. This chapter is followed by the results of the four data collection methods (websites review, interviews with health professionals, patients and parents, and surveys of patients and parents) in Chapters 6, 7, 8, and 9. 
Part three is devoted to synthesis of the evidence across the entire thesis. I begin in the discussion chapter by merging the pieces of evidence from the four chapters reporting the findings, noting similarities and differences, and examining them in the light of previous research in SCD self-manage. I then summarise the contributions produced by the thesis by relating the results to the aim of the study. I further outline the implication of the study to practice, theory and future research in SCD self-management. 


\section{Chapter 2: Overview of SCD and Management in Ghana}

This chapter provides a general overview of SCD, the current management, and management in the Ghana health system. The chapter is set out in two parts. The first part, which provides an overview of SCD, consists of six sections. The first section provides general knowledge of SCD. This section situates SCD within the global categorisation of severe inherited haemoglobinopathies, and describes the mode of inheritance, and common types. The second section distinguishes between SCD and Sickle Cell Trait (SCT), and discusses the pathological implications for SCT. The third section describes the aetiology and epidemiology of sickle cell in terms of origin, global distribution and occurrence within the Ghanaian context, and justifies the relevance of SCD research in Ghana. The fourth section describes the pathophysiology, clinical features, natural history, and clinical trajectory and compares these with SCD presentation in Ghana. The fifth section describes the psychosocial burden of SCD and implications for patients and families. Finally, the sixth section focuses on the treatment and management of SCD and highlights the role of nurses, patients and families. The second part of the chapter provides an analysis of current health services for SCD management in the Ghana Health System. It includes a further discussion of the gaps in services that were outlined in Chapter one.

\subsection{Overview of Sickle Cell Disease}

SCD is the world's most common severe inherited haemoglobinopathy. Severe inherited haemoglobinopathies, predominantly SCD and thalassaemia, are issues of international health concern for $71 \%$ of 229 countries [1]. An estimated 300,000 babies are born annually with these disorders, $85 \%$ of whom are found in the middle and low income countries of which $70 \%$ are in Africa $[1,65]$. An estimated $24 \%$ of the world's population carry abnormal haemoglobin gene variants, which translates to affected birth prevalence of about 2.55 per 1000 live births. SCD accounts for $75 \%$ of children born with haemoglobinopathies and is responsible for $80 \%$ of the burden of haemoglobinopathies [1, 66]. Whereas most affected children with severe haemoglobinopathy survive in high income countries, in low income countries the majority die before age five. Severe inherited haemoglobinopathies contribute an equivalent of $3.4 \%$ of mortalities in children under five worldwide and $6.4 \%$ in Africa [1]. 
SCD is a genetic disorder of the red blood cells characterised by the production of an abnormal haemoglobin: haemoglobin $\mathrm{S}(\mathrm{HbS})$ [67]. This disorder is transmitted through autosomal-recessive inheritance of the sickle gene (faulty gene) [68]. The sickle gene results from substitution of glutamic acid by valine on the sixth position of the beta globulin chain, resulting in the production of haemoglobin $\mathrm{S}(\mathrm{HbS})[16,69]$. The disease state occurs when individuals inherit the homozygous $\mathrm{HbS}$ or co-inherit $\mathrm{HbS}$ with other abnormal haemoglobin variants, especially haemoglobins C, E, D Punjab; or with the thalassaemia gene - beta thalassaemia or alpha zero thalassaemia $[1,69]$. Sickle cell anaemia technically refers to the genotype HbSS or HSbeta zero thalassaemia [70] whereas sickle cell disease refers to the other genotypes including Sickle Haemoglobin C Disease (HbSC) and Sickle Beta zero thalassaemia $\left(\mathrm{HbS}^{0}\right.$ thalassaemia). In this thesis, SCD refers to all genotypes. The severity of the condition differs by genotype ranging from mild to severe, and the various genotypes have different geographical distribute (Table 1). The common genotypes in Ghana are HbSS, $\mathrm{HbSC}, \operatorname{HbS} \beta^{0}$ thalassaemia and $\operatorname{HbS} \beta^{+}$thalassaemia [59].

\subsection{Sickle Cell Trait and Implication}

Individuals who inherit $\mathrm{HbS}$ from one parent and a normal haemoglobin gene A ( $\mathrm{HbA}$ ) from the other have the sickle cell trait (SCT). Such people have the genotype (HbAS) and are referred to as a sickle cell carrier [65]. The condition is generally asymptomatic and many carriers are unaware of their status, and often are diagnosed through incidental blood tests [69]. Individuals with the SCT have the risk of transmitting the sickle gene to their offspring and can potentially produce children with SCD, if their mating partners are carriers or have SCT [65]. The inheritance of the SCT is believed to be associated with beneficial and deleterious effects. The beneficial effect results from the ability to provide resistance to severe falciparum malaria and consequently, those with SCT have longer life expectancy than HbAA and HbSS counterparts in malaria endemic.

The MOH of Ghana considers SCT a benign condition with comparable haematological indices to people with normal haemoglobins. However, it continues to monitor developments regarding its pathology to inform future decision [4]. This thesis is concerned with people with SCD rather than SCT. 
TABLE 1. COMMON FORMS OF SCD, CHARACTERISTICS AND GEOGRAPHICAL DISTRIBUTION

\begin{tabular}{|c|c|}
\hline Type of SCD & Different types of SCD and Geographical Occurrence \\
\hline \multicolumn{2}{|l|}{ Severe sickle cell disease } \\
\hline $\mathrm{HbSS}$ & The commonest form of sickle cell disease \\
\hline $\mathrm{HbS} \beta^{0}$ thalassaemia & Most prevalent in the Eastern Mediterranean region \& India \\
\hline Severe $\mathrm{HbS} \beta^{+}$thalassaemia & Most prevalent in the Eastern Mediterranean region \& India; $1-5 \% \mathrm{HbA}$ present \\
\hline HbSO Arab & Reported in North Africa, the Middle East \& the Balkans; relatively rare \\
\hline HbSD Punjab & Predominant in northern India but occurs worldwide \\
\hline HbSC Harlem & $\begin{array}{l}\text { Electrophoretically resembles } \mathrm{HbSC} \text {, but clinically severe; double mutation in } \\
\beta \text {-globin gene; very rare }\end{array}$ \\
\hline HbC Antilles & $\begin{array}{l}\text { Double mutation in } \beta \text {-globin gene results in severe sickle-cell disease when co- } \\
\text { inherited with } \mathrm{HbC} \text {; very rare }\end{array}$ \\
\hline HbS Quebec-CHORI & $\begin{array}{l}\text { Two cases described; resembles sickle-cell trait with standard analytical } \\
\text { techniques }\end{array}$ \\
\hline \multicolumn{2}{|l|}{ Moderate sickle cell disease } \\
\hline $\mathrm{HbSC}$ & $25-30 \%$ cases of sickle-cell disease in populations of African origin \\
\hline $\begin{array}{l}\text { Moderate } \mathrm{HbS} \beta^{+} \\
\text {thalassaemia }\end{array}$ & Most cases in the Eastern Mediterranean region; $6-15 \% \mathrm{HbA}$ present \\
\hline HbAS Oman & $\begin{array}{l}\text { Dominant form of sickle-cell disease caused by double mutation in } \beta \text {-globin } \\
\text { gene; very rare }\end{array}$ \\
\hline \multicolumn{2}{|l|}{ Mild sickle cell disease } \\
\hline Mild $\mathrm{HbS} \beta^{++}$thalassaemia & Mostly in populations of African origin; $16-30 \% \mathrm{HbA}$ present \\
\hline $\mathrm{HbSE}$ & $\begin{array}{l}\text { HbE predominates in Southeast Asia \& so HbSE uncommon, although } \\
\text { frequency is increasing with population migration }\end{array}$ \\
\hline HbA Jamaica Plain & $\begin{array}{l}\text { Dominant form of SCD; double mutation results in } \mathrm{Hb} \text { with low oxygen } \\
\text { affinity; one case described }\end{array}$ \\
\hline \multicolumn{2}{|l|}{ Very mild sickle cell disease } \\
\hline HbS HPFA & $\begin{array}{l}\text { Group of disorders caused by large deletions of the } \beta \text {-globin gene complex; } \\
\text { typically } 30 \% \text { foetal Haemoglobin }\end{array}$ \\
\hline HbS Other Hb variants & $\begin{array}{l}\text { HbS is co-inherited with many other Hb variants, and symptoms develop only } \\
\text { in extreme hypoxia }\end{array}$ \\
\hline
\end{tabular}

Source: Rees, D.C., Williams, T.N., \& Gladwin, M.T. (2010). Sickle-cell disease. Lancet, 376(9757), 2018-2031. Doi: 10.1016/s0140-6736(10)61029-x 


\subsection{Aetiology and Epidemiology of Sickle Cell Disease}

The first scientific report of SCD dates back to 1910 when a Chicago cardiologist James Herrick (1861-1954) discovered large numbers of elongated sickle and crescent shaped red blood corpuscles in the blood films of Walter Noel, a 20-year-old dental student from Grenada [71]. The sickle cell mutation is reported to have originated independently in five geographical areas in Africa and Asia [72]. However, the gene is currently spread to most regions of the world due to human migration $[1,65]$. The trans-Atlantic slave-trade between 1451-1870 is claimed to be one of the initial causes of the spread [54]. The current global distribution of $\mathrm{HbS}$ affects many nations in varying magnitudes. The gene is most common among people with ancestry from African, Indian, and the Middle-East and Mediterranean regions [3]. The overall global picture shows that Africa has the highest burden with subSahara Africa being the worst affected [1, 54, 65, 73].

Historically, estimation of the global prevalence and incidence of SCD has been a major challenge. It has been argued that the most appropriate way to obtain the precise numbers with $\mathrm{HbS}$ is through universal neonatal screening programmes Piel, Patil [65]. However, because many nations, especially with lower financial resources, lack screening programmes, global estimates have been based on conservative estimates, usually based on the prevalence among new-born and affected pregnancies $[1,65,74]$. Therefore, there is limited information of the prevalence of SCD among the general population in many nations $[3,73]$.

The first rigorously evidence-based contemporary estimates of global prevalence of $\mathrm{HbS}$ (HbSS and HbAS) among new-borns was the Piel et al. [65] publication. In this study, the researchers combined databases of sickle haemoglobin surveys and created a contemporary global map of $\mathrm{HbS}$ allele frequency distribution within a geostatistical model. Based on this model, the researchers calculated the global, regional, national, and sub-nation estimates of annual numbers of $\mathrm{HbSS}$ and $\mathrm{HbSS}$ neonatal births. The findings are presented based on WHO country groupings (WHO regions) and haemoglobin S regions (researcher's defined regions [Table 2]).

Piel et al. [65] reported that in 2010, the number of babies born HbS worldwide was approximately 5,788,709. This included 312,302 HbSS and 5,476,407 heterozygous HbAS 
babies. The highest frequencies of HbS occurred in Sub-Sahara Africa. The other areas with high frequencies were the Middle East and India. Half of the births were located in three countries: Nigeria $(1,223,330)$, India $(1,038,579)$ and the Democratic Republic of Congo $(527,953)$.

Table 2. AnNual Global Predicted Estimates of HB AS and HB SS among Neonates

\begin{tabular}{|l|r|r|r|}
\hline & $\begin{array}{l}\text { Average HbAS } \\
\text { Neonates/year }\end{array}$ & $\begin{array}{l}\text { Average HbSS } \\
\text { Neonates/year }\end{array}$ & \multicolumn{1}{l|}{ Total } \\
\hline WHO Regions & $3,607,022(64.2 \%)$ & $239,545(74.5 \%)$ & $3,846,567(64.2 \%)$ \\
\hline Africa & $1,040,033(20.0 \%)$ & $44,132(15.1 \%)$ & $1,040,033(20.0 \%)$ \\
\hline South East Asia & $398,279(7.6 \%)$ & $137,089(4.6 \%)$ & $398,279(7.6 \%)$ \\
\hline The Americas & $275,365(5.7 \%)$ & $10,007(3.6 \%)$ & $275,365(5.7 \%)$ \\
\hline East Mediterranean & $127,494(2.6 \%)$ & $3653(1.3 \%)$ & $127,494(2.6 \%)$ \\
\hline Europe & $2,292(0.0 \%)$ & $4(0.0 \%)$ & $3,607,022(64.2 \%)$ \\
\hline West Pacific & $3,579,982(64.4 \%)$ & $237,253(75.5 \%)$ & $3,817,235(66.7 \%)$ \\
\hline Haemoglobin S region & $1,168,805(22.7 \%)$ & $48,951(16.9 \%)$ & $1,217,754(21.3 \%)$ \\
\hline Sub-Sahara Africa & $389,892(7.4 \%)$ & $13,309(4.6 \%)$ & $403,201(7.0 \%)$ \\
\hline Arab-India & $271,474(5.4 \%)$ & $8784(3.0 \%)$ & $280,258(4.9 \%)$ \\
\hline Americas & $4,854(0.1 \%)$ & $80(0.0 \%)$ & $4,934(0.1 \%)$ \\
\hline Eurasia
\end{tabular}

Adapted from: Piel et al. (2013). Global epidemiology of sickle haemoglobin in neonates: A contemporary geostatistical model-based map and population estimates. Lancet, 381(9861), 142-151

\subsubsection{The Ghanaian Context}

The WHO estimates that sickle cell affects about 40 countries on the African continent in varying magnitudes [73]. It is more common in the Eastern, Central and Western parts of the continent and lowest in the Northern and Southern parts $[65,73]$. The sickle cell epidemiology in Ghana is similar to that of other West African countries with approximately 16,000 to 18,000 SCD babies born per annum. Approximately $90 \%$ of these babies are SS and SC [58]. The NSSCD Programme implemented in 19 public and private health facilities in Kumasi and Tikrom in the Ashanti region, in 1993 shows that by December 2011, a total of 342,355 babies had been tested of which 6,025 (1.8\%) were identified with SCD [75].The screening report also showed that approximately $25 \%$ have SCT (AS and AC).

\subsection{Pathophysiology and Clinical Features}

SCD is characterised by a clinical syndrome affecting multiple organs. The clinical features are underlined by a complex pathophysiological process involving vaso-occlusion 
and rapid haemolysis [76]. Red blood cells (RBCs) containing HbS have the tendency to become crystallised, deformed, hard and brittle when deoxygenated [77]. Deformed RBCs cause vascular obstruction and tissue ischaemia leading to pain and tissue damage [77, 78]. HbS polymerisation also causes haemolytic anaemia [77].

People with SCD experience a continuum of signs, symptoms and complications from mild to life-threatening and clinical features are defined mainly by haemolytic anaemia and sequels, pain syndrome, multiple organ complications and sepsis $[16,70]$. Pain syndrome is the commonest problem. It can be acute, subacute, chronic or episodic [5] and can be spontaneous or triggered by factors such as cold weather, rigorous exercise, infection, dehydration, alcohol consumption, low of oxygen levels, stress, fatigue, pregnancy and air travel [77]. The intensity and duration may be influenced by a variety of factors including, biochemical, neurological and psychological, cultural, spiritual and environmental [5].

SCD is also associated with chronic anaemia, which differs in severity by genotype. Patients with $\mathrm{HbSS}$ and $\mathrm{HbS} \beta^{+}$thalassaemia experience a severe form of anaemia. Anaemia may be complicated by a variety of factors such as hyper haemolysis, acute splenic sequestration and aplastic crisis. Several other complications may result from chronic anaemia and vaso-occlusion affecting multiple organs. Children with SCD also experience growth retardation with decreased height, weight and body mass index compared to their healthy counterparts [5].

The complications of SCD can be acute or chronic. Acute complications are characterised by sudden unpredictable onset, usually without identified cause. Vaso-occlusive crisis is commonly referred to as "painful crisis" and is the most common. Other acute pain syndromes include acute chest syndrome (ACS), acute stroke, priapism, hepatobiliary complications, splenic sequestration and acute renal failure [70, 79].

Infections are also major acute problems among children under five years. SCD children usually experience reduced or absent splenic function by 2 to 3 months of age rendering them susceptible to severe bacterial infection, especially Streptococcus pneumonia, non-typhi salmonella species and malaria $[16,70]$. Fever may also occur due to incomplete vaccination, penicillin resistant infections, defects in complement activation, micronutrients deficiency and tissue ischaemia [79]. Fever is usually associated with acute chest syndrome, 
urinary tract infections, and hepatobiliary system or bone infections [70]. Fever above $38.5^{\circ}$ Celsius constitutes an emergency that demands prompt therapy to treat S. pneumonia and most gram negative enteric organisms. Mortality from infection greatly reduced due to early implementation of penicillin prophylaxis and vaccinations for Haemophilus influenzae and Streptococcal pneumoniae [16].

Chronic complications may affect SCD patients during their entire life-span, or may result from unresolved acute complication or under-treated complications. Chronic pain is the most common and is usually debilitating both physically and psychologically. Other complications include avascular necrosis, pulmonary hypertension, renal complications, ophthalmic complications, leg ulcers, stuttering or recurrent priapism. The severity of complications varies amongst individuals and overtime. Because complications increases with age, knowledge of precipitation factors and predictors are important for reducing morbidity $[70,79]$.

\subsubsection{Natural History and Clinical Trajectory and Prognosis}

The manifestations of SCD are age dependent, differ from person to person and begin from about 8-10 weeks of life. The first year of life is the most critical. Symptoms begin as foetal $\mathrm{Hb}$ decreases from birth and replaced by $\mathrm{HbS}$ [72]. The first two months are usually asymptomatic and symptoms typically commence between 2-6 months. Dactylitis starts between 10-12 weeks and affects $50 \%$ of children with SCD. This may resolve spontaneously after 5-7days with analgesics and becomes rare after five years [80]. Dactylitis requires explanation to parents as people can associated this with child abuse. Other early complications are acute chest syndrome, abnormal spleen function rendering the infant susceptible to overwhelming infections, septicaemia and acute splenic sequestration. Early diagnosis, preferably through screening, is important to commence a treatment programme $[72,80]$. The second six months presents the highest risk of mortality due to overwhelming infections, sepsis and ACS. As acute splenic sequestration is common between six months to two years, the role of parents in splenic palpation helps assists early diagnosis and interventions [72].

Between one and 14 years, SCD children are more likely to experience stroke in addition to the other problems. The median onset of stroke is six years and occurs in $8 \%$ of 
patients by fourteen years that may cause death or interfere with study and employment. Hypersplenism is characterised by persistent splenic enlargement occurring in about $5 \%$ of this age group. It may resolve spontaneously or is treated with splenectomy. Aplastic crisis is another common problem and is usually caused by par virus B19. Sequestration crisis is more common in $\mathrm{SS}$ and $\mathrm{HbS}^{+}$thalassaemia $[72,80]$.

Common problems during adolescence and early adult life are enuresis, leg ulcers, increased bone pain, decreased growth and sexual development, priapism and hip disease. A second peak of stroke may happen at about 25 years and is predominantly haemorrhagic with poor prognosis. Increased incidence of ACT and resultant pulmonary hypertension occur during this period. Patients at this stage require major clinical and psychosocial support.

Many of these problems may continue throughout adult life and can worsened during pregnancy. These include retinopathy, aseptic necrosis, leg ulcers, cholecystitis, and liver disease. Bone pains may subside between 25-30 years and patients experience better QOL [72]. After age 40 years, renal impairment with associated decrease in Hb levels is more prominent and may result in cardiac problems. Organ damage is common in adult life [72, 80]. The typical clinical picture of SCD in Ghana is presented in Table 3. This picture is similar to what is reported in international literature.

The clinical expressions and prognosis of SCD depend on the presence of genetic, physiological, and environmental factors [72, 81]. Genetic factors such as inheritance of genes for Hetero-cellular Hereditary Persistence of Foetal Haemoglobin, co-inheritance of $\alpha$ thalassaemia, and low Mean Cell Haemoglobin Concentration lead to milder conditions [72, 82]. People with genotypes $\mathrm{HbSS}$ and $\mathrm{HbS} \beta^{+}$thalassaemia have a more serious condition compared with people with $\mathrm{HbSC}$ or $\mathrm{HbS}^{+}$thalassaemia [83]. Physiological factors that affect clinical expressions of SCD include abrupt changes in temperature, hypoxia, dehydration, infection, fever, physical exertion, pregnancy, and psychological stress [81, 84]. Environmental factors including climatic factors that result in skin cooling and socioeconomic factors including nutrition, and availability of public health measures such as immunisation, access to specialised medical care and parental or social support can also influence the clinical course of SCD $[54,72,85,86]$. 
Without intervention, SCD usually causes disabilities and premature mortality. This occurs commonly among children under five years, adolescents and pregnant women [61]. The WHO [61] indicates that in the absence of basic interventions, between $50 \%$ and $80 \%$ of children with SCD die before age five usually from infections or severe sepsis. SCD contributes an equivalent of 5\% under 5-year deaths in Africa, 9\% in West Africa and up to $16 \%$ in individual West African countries [87]. Children who live beyond age five have the risk of premature death due to exacerbations of the disease and the presence of complications. Table 3 presents a summary of age-related manifestations for the Ghanaian context.

TABLE 3. AGE RELATED MANIFESTATIONS OF SCD IN GHANA

\begin{tabular}{|c|c|}
\hline Age Group & Manifestation \\
\hline \multirow{7}{*}{$\begin{array}{l}\text { Infancy and } \\
\text { childhood } \\
0-14 \text { years }\end{array}$} & Sickle cell dactylitis \\
\hline & Severe sepsis-pneumococcal, meningococcal, \& H influenza \\
\hline & Malaria \\
\hline & Acute splenic sequestration syndrome \\
\hline & Acute osteomyelitis-staphylococcal \\
\hline & Stroke \\
\hline & Enuresis \\
\hline \multirow{6}{*}{$\begin{array}{l}\text { Adolescence } \\
15-30 \text { years }\end{array}$} & Vaso-occlusive crisis \\
\hline & Priapism \\
\hline & Leg ulcers \\
\hline & Somatic growth retardation \\
\hline & Psychosocial problems \\
\hline & Schooling problems \\
\hline \multirow{7}{*}{$\begin{array}{l}\text { Adults } \\
31+\text { years }\end{array}$} & Vaso-occlusive crisis \\
\hline & Chronic osteomyelitis \\
\hline & Leg ulcers \\
\hline & Gall stones \\
\hline & Pregnancy morbidity and mortality \\
\hline & $\begin{array}{l}\text { End organ damage (proliferative retinopathy, avascular } \\
\text { necrosis of femoral head, gall stones, impotence, renal failure) }\end{array}$ \\
\hline & Unemployment \\
\hline All ages & Aplastic crisis \\
\hline
\end{tabular}

Survival with SCD has improved due to advances in management, especially with the introduction of vaccination against pneumococcus. Mortality among black African children under four years decreased by 42\% between 1999 and 2002 in USA [88]. Life expectancy also increased from an estimated 14.3 years in the 1960 s to 42 years and 48 years respectively 
for males and females with HbSS, and 60 years and 68 years respectively for males and females with HbSC. Many SCD patients lived beyond their $5^{\text {th }}$ decade in the $1990 \mathrm{~s}$ in the USA [89]. In Jamaica, the median survival was estimated to be 53 years and 58 years for males and females respectively [90]. Both the US and Jamaica have Comprehensive Health Care and Management for SCD [72]. Accurate data on life expectancy of SCD patients in Africa is unavailable but the WHO estimates mortality is higher considering limited access to essential health services. Estimates based on the age structure of populations attending clinics indicate that half of the infants born with SCD died by age five [87]. In most poorly-resourced countries, including Ghana, the clinical cause and natural history is poorly understood due to limited research. In Africa, it is believed that many undiagnosed SCD patients die from preventable causes such as malaria, infections, and severe acute anaemia [16, 54].

Understanding the pathophysiologic, clinical features and disease trajectory is important for patients' self-management. These have implications for patients' appreciation of factors that underline exacerbation of the disease, the dynamics of the disease, and the actions required at each stage of the disease process to ameliorate complications.

\subsection{Psychosocial Burden of Sickle Cell Disease}

In addition to the physical problems, SCD patients and families experience significant psychosocial burden that impacts on outcomes. These are considerable in countries with low financial resources and limited access to welfare and health services [91]. Psychosocial concerns in SCD are influenced by the impact of the disease on the patient and society's attitudes towards the patients and families [92]. The concerns from impacts of disease include recurrence of painful crisis, physical deformities, illness and thoughts of death at any time, and frequent absence from school with poor academic performance [93]. Psychosocial burden also results of treatment of SCD such as attending clinical appointments and waiting times, taking daily medication, frequent hospitalisation with blood transfusion and attitudes of hospital staff [91, 93]. Additional burden results from the direct financial cost of SCD care including the cost of drugs, blood, hospital billing, nutrition and transportation to clinic; and indirect financial cost due to loss of income through decreased work hours, work performance, and time spent in providing care [91, 93]. 
Public perception, lack of social services and other external factors determine the extent to which psychosocial factors impact on patients and families. In Ghana, for example, many lay perceptions of the pathology of SCD are derogatory and associated with discourses of death [94]. The presence of a family member with SCD can also create disturbances to routine family activities and interactions creating tension, hostility, disagreement, marital disharmony, embarrassment and stigmatisation [91, 93]. Disruption within the family adversely impacts on the life of the SCD patient who may lack sufficient help or support from spouse and other family members translating into depression or anger decreasing QOL [91].

Adults, adolescents and children have unique psychosocial problems [92]. Adults are commonly confronted with marital dysfunction, employment problems, fear of body deterioration, and stigmatisation that translate into deterioration of social networks. Adolescent problems relate to social functioning, stigmatisation and transition to independent adult life. The major concerns for children are schooling and other scholastic challenges [95].

These psychosocial concerns create additional burden to patients and families, influencing the clinical course of the disease [96]. The increasing life expectancy of SCD patients, including children, will require a holistic approach to management involving selfmanagement and psychosocial interventions.

This section focused on the pathophysiology, resultant clinical expressions, disease trajectory and implication for physical and psychosocial functioning of the SCD patient. The next section will focus on the treatment of SCD complications.

\subsection{Treatment and Management of SCD}

Many SCD related problems can be managed to improve patients QOL. As SCD can affect multiple organ systems, the management transcends multiple medical specialities. Best practice indicates that SCD is ideally managed at specialised units by a multidisciplinary teams of specialist including doctors, nurses, social workers, health educators and genetic counselors facilitating in-patient, outpatient and follow-up care [16]. These services are delivered in comprehensive SCD centres in the USA and UK [16, 70]. No such centres currently exist in Ghana. 
Following diagnosis, the goal of SCD management is to maintain patient health, and prevent or manage complications. Ballas, Kesen [5] categorises the management of SCD into five areas: supportive, symptomatic, preventive, abortive and curative. Supportive management is aimed at maintaining good health through optimising diet, sleep, hydration, folic acid supplementation and regular follow-up. Symptomatic management focuses on alleviating the symptoms of the disease using blood transfusion for anaemia, analgesics for pain and antibiotics for infections. Preventative management approaches include vaccination, avoidance of stressful situations, $\mathrm{HbF}$ induction with $\mathrm{HU}$ or related medicines and blood transfusion to prevent stroke. Abortive management involves treatments to prevent crises escalating and include nitric oxide and anti-adhesion factors administration. Curative care involves bone marrow transplantation.

Many authorities categorise SCD management into health maintenance, general management, and management of specific problems $[16,77,79]$. In the following section, I discuss the management under these headings and describe the role of nurses, patients and families.

\subsubsection{Health maintenance}

Health maintenance management includes patients-focused education, continuous health care, preventive treatment, counselling, and special health assessment. The procedures involved differ between children and adults. As only one publications was identified regarding health maintenance management [79], this section is informed by this USA publication.

Child health maintenance involves parental education of physical assessment skills such as splenic palpation, administration of prophylaxis including penicillin and folic acid, strategies to avoid crises, and how to navigate the medical system. The education covers fever and pain recognition and home management. Parents are also counselled on nutrition including avoidance of iron supplements, correction of nutritional deficiencies, and use of fluoride in water to prevent dental decay. In addition, the counselling involves academic, vocational, recreational and air travel precautions. The SCD child also requires continuous healthcare at the well-baby clinic for growth monitoring, immunisation (especially pneumococcal vaccination), and counselling in preventive measures. 
Special physical laboratory and other investigations are conducted on children. Parents are informed of typical SCD features including jaundice, protruded abdomen, anaemia, cardiac systole flow murmur, maxillary hypertrophy with overbite, delayed physical growth and development and short stature, and delayed reproductive health development. Baseline laboratory values are also obtained for future comparison. Investigations are routinely conducted on organs that can be damaged from SCD, especially the lungs and the brain. These include lung function tests to monitor ACS, transcranial doppler imaging and magnetic resonance imaging to monitor the risk of stroke, silent infarction and neuropsychometrics.

Health maintenance in adult patients addresses medical and psychosocial issues of SCD and the interactions with age-related co-comorbidities such as hypertension and cancer. Patient education involves identifying and addressing knowledge deficits, identifying emergency signs and symptoms and managing pain at home. Continuous care includes occupational and physical therapy such as behaviour modification, neurological interventions and counselling on nutrition and lifestyle including alcohol and tobacco use. Counselling also includes folic acid supplementation, avoiding precipitating factors, contraception, preconceptual genetic counselling, choosing safe occupations, legal protection against discrimination and other work related policies, dental and psychosocial care. Patients may also require vaccinations for infections such as tetanus, hepatitis and influenza. Special evaluation includes annual haematological assessment, pulmonary function test, and ophthalmic examination.

\subsubsection{Treatment of complications}

The treatment of SCD related illnesses and complications are based on presenting symptoms. Despite the possibility of a cure through allogeneic hematopoietic stem cell transplantation [97], SCD management is largely supportive [77]. The treatment of SCD problems are classified as either general or specific. The general classification involves interventions that modify the pathology of the disease to improve the clinical outcomes. The most established of these interventions, with wide international consensus, are hydroxyurea therapy and red cell transfusion [16, 98-100].

Acute complications require immediate evaluation and treatment to reduce or prevent morbidity and mortality, and preserve organ function [100]. Pain relief, hydration, 
management of sepsis and blood transfusion are the key strategies for acute management [16]. Pharmacological measures used to manage acute pain are analgesics, mainly opioids, NSAIDs and adjuvants such as sedatives and anxiolytics [100]. Non-pharmacological measures may include spirometry, oxygen administration, messages, muscle relaxation therapies, selfhypnosis, baths and distraction strategies. Blood transfusion may be given for debilitating pain $[79,100]$.

Chronic complications can affect any organ and chronic pain is the commonest. The aim of treatment is to minimise pain, increase coping, improve social and physical functioning, improve quality of life and prevent organ damage [100]. In addition to the use of analgesia, other interventions include occupational and physical therapies, behaviour modification and neurocognitive therapies are utilised in managing chronic complications [79].

The management of SCD also involves psychosocial interventions including cognitive behaviour therapy (CBT), self-regulated therapies, behaviour strategies, social support interventions, education and medical self-management. CBT has received the most empirical support in the literature but the successful delivery of CBT relies on qualified expert mental health professionals [101]. While these interventions are integrated into comprehensive care and management in countries with high financial resources, there is paucity of these interventions in countries with low financial resources including Ghana [54, 101]. Therefore, a country such as Ghana needs to consider locally suitable alternative interventions to assist patients and families to address psychosocial issues of SCD; and this must include selfmanagement.

\subsubsection{The role of the nurse in SCD management}

Nurses provide a significant role in SCD management. The nurses' role in SCD depends on whether they are SCD specialist or generalist, and whether the client is in the clinical or community setting [68]. The duties of the specialist nurse include initial assessment of patients in general haematology clinics, running nurse-led clinics, administering desferrioxamine (iron chelators), performing exchange blood transfusions, collecting essential data for the SCD database, maintaining the SCD patient register, and other nursing duties 
[102-104]. Countries such as US and UK have certified specialised SCD nurses [105]. Ghana presently does not train certified SCD specialist nurses.

The roles of nurses, both specialist generalist, in the clinical setting involves the prompt treatment of acute problems, instituting preventative measures to avoid adverse clinical outcomes, and instituting long-term measures for disease management and comfort that will allow patients to self-management at home [67]. Nurses require special competencies for clinical management of SCD (Table 4).

TABLE 4. TEN COMPETENCIES OF THE NURSE IN MANAGING SCD

Provides empathy and understanding, and works with the patients ( $\&$ their family/carer) as expert in their own condition

Assesses the needs of patients, taking into account the impact on their age and developmental stage, and their cultural ethnic background Undertakes comprehensive physical assessment and follow-up, including referral to medical specialist for relevant chronic healthcare conditions

Signposts and supports patients ( \& families/carers) in their understanding of their genetic conditions

Develops and evaluates a self-management plan with the patient

Works alongside and with the patient (\& family/carers) to address the psychological and social impact of their condition

Works with the patient (and family/carers) to manage their pain

Provides specific interventions safely with regards to phlebotomy, cannulation, central

venous devices, portacaths, transfusion; fluid management and hydration;

pharmacological treatment and side effects; iron overload management including

chelation therapy

Uses early warning tools/approaches (e.g., red alert) to identify the patient's changing and deteriorating condition, and takes appropriate action

Actively improves and promotes services across the care pathway

Source: Tangayi, S. (2011). Framework for thalacaemia and sickle cell disease.

Retrieved from http://www.nursingtimes.net/nursing-practice/clinical-zones/long-term-conditions/-a-skills-

framework-for-sickle-cell-disease-and-thalassaemia

Nurses also promote long term and home management through patient and family education. Patients' education helps to maximise the benefits of clinical care, boost parental confidence in caring for children, and enhances patients' ability to live with the disease [62]. The education concerns medication administration and side effects; nonpharmacological pain management techniques; recognition of emergency signs and precipitating factors, hard facts about SCD, health maintenance, lifestyle modification, and clinical attendance $[62,67]$. 
Education of caregivers on splenic palpation and measurement is instrumental in early identification of splenic problems. Varieties of educational materials and approaches including print and audio-visual materials are used by nurses [62]. The use of educational guide and other protocols has been found to enhance empowerment of patients and caregivers to manage their long-term illnesses [106].

Community care by nurses is vital in the management of SCD for several reasons. Many SCD patients are managed at home especially when they are not experiencing crises and others chronic disabling complications [95]. Furthermore, SCD has deleterious effects on children's school attendance and educational performance. Adults have issues with employment and other social problems [107]. The role of the nurse in the community is to provide a link between the patient's home and the hospital or community-based services. They work with providers of various services to address the psychosocial and medical issues faced by SCD patients in their domestic and community settings. Collaborators include general practitioners, community physiotherapists, community occupational therapists, adult disability teams, social workers, housing officers, community council staff who provide home help, rehabilitation centres, visual impairment teams and voluntary agencies [102]. Community care also enables the nurse to reinforce education and support for prophylactic and other general maintenance measures $[62,108]$. Obviously, nurse play pivotal role in SCD management at the clinical and community setting. Through collaboration with other health care providers and community partners, nurses help to address the medical and psychosocial concerns of SCD patients both at the clinical and community settings. Majority of the nurses' roles especially regarding home and community relates to patients self-management. Therefore, the nurses' role can be enhanced or redirected to improve patients' selfmanagement and general care of SCD patients.

\subsubsection{Patients' and families' responsibilities in SCD management}

A number of authors have indicated the importance of patients and families contribution to the management of SCD related problems [16, 68, 79]. These authors recommend health professionals include helpful self-management practices patients already use in planning care. However, most literature focus on the responsibilities of health professionals. In the literature where information on patient's responsibilities are provided, 
the focus are on health maintenance and prevention of painful crises with little information regarding patients' responsibilities for managing complications. There are limited recommendations on patients' responsibilities for complications including fever, pain, leg ulcers, and splenic enlargement National Heart Lung \& Blood Institute [79].

Patients' and families' responsibility in health maintenance include physical assessment such as splenic palpation and temperature, understanding and keeping results of physical findings and laboratory values, and engaging in medication administration. It also involves avoiding triggers of disease exacerbation such as exhaustion, dehydration, and extremes of temperature. Among children, parental responsibility involves taking the child to well-baby clinics, administering prophylactic penicillin, adhering to immunisation and informing school teachers and child's school friends about the child's condition. Among adolescents and adults, patients' responsibilities involve safe sexual behaviours, use of contraception, awareness of pregnancy risks and choosing occupations compatible with SCD.

Patients' and families' roles in the management of complications are to monitor signs and symptoms, report dangerous signs and symptoms or deviations such as fever, chest pains, difficulty in breathing, atypical pain and severe pain. Furthermore, patients' responsibilities include following medical instructions, keeping clinical appointments and undertaking requested investigations. Patients and families role further includes learning the common signs and symptoms specific to their condition and the recommended actions when such signs and symptoms are recognised [79].

Regarding specific complications, the National Heart Lung \& Blood Institute [79] indicates that patients experiencing avascular necrosis of the femoral head should reduce weight. Patients on blood transfusion therapy should to be aware of iron overload and the need to use iron chelators. Furthermore, pregnant women on hydroxyl urea are required to be aware of the need to withdraw from the medicines during pregnancy as it is cytotoxic. Patients' responsibility in pain management involves pain assessment, use of pain diaries, home treatment and reporting pain that is unsuccessfully managed. Patients using opioids are required to monitor the side effects and take stool softeners. Patients treated with ulnar boots for leg ulcers are taught to change their dressing and ensure complete bed rest with limb elevation. Male patients who experience priapism should avoid a full bladder and prolonged 
sexual activity. As SCD patients, especially those with $\mathrm{HbSC}$ and $\mathrm{HbS} \beta^{+}$thalassaemia, are prone to eyes complications, patients are advised to have annual ophthalmic evaluation and report eye traumas or acute change of vision to an ophthalmologist.

Patients and their families therefore have key roles in all areas of SCD management. Although best practice indicates that health professionals must recognise the contribution of patients managing their disease, the roles and responsibilities for patients form the foundation of self-management in SCD, especially the management of acute and chronic complication that have limited discussion in the literature. So far this section has provided a description of SCD and its management internationally; management should include interventions that are anticipatory, preventive, promotive, reactive and rehabilitative.

\subsection{SCD Management in the Ghanaian Health System}

This section presents an overview of the current status of SCD management in the Ghana health system. These services are analysed within the context of the WHO recommended strategies for SCD management for the African region [73] and Ghana's strategic framework [4]. The section draws on policy documents and my personal experiences.

The WHO [73] recommends that African countries with high prevalence of SCD create or strengthen national SCD programmes with components including: early identification through screening of new-born and targeted screening for carriers and pregnant women, dedicated centres for prevention and treatment, capacity building of health professionals through pre-service and in-service training, supportive care actions, and primary prevention through genetic counselling and testing to reduce partnering of carriers. The WHO further advises that patients' management must be based on Comprehensive Health Care and Management (CHAM). In the African region, CHAM must comprise parents and patients' education, adequate nutrition, adequate hydration, use of prophylactic antibiotics and antimalarias, folic acid supplementation, use of specific vaccines, continuous medical follow-ups, and early detection and management of complications. The CHAM services must also include: interventions to prevention complications, patient referral, provision of medicines for pain management, strengthening of laboratory and diagnostic capacity, and research and surveillance. The WHO further recommends that such interventions must be integrated into 
health systems using the primary health care approach to meet the needs of both rural and urban dwellers, and must include family and community-based services. The WHO asserts that these measures can reduce morbidity, prevent complications and improve the quality of life for SCD patients.

In line with WHO recommendation, Ghana developed a national SCD strategy including advocacy, prevention, counselling, early detection, management and follow-up, data collection, surveillance and research, and community education. Ghana has made limited progress towards implementing the national strategy for SCD. Attempts to officially recognise SCD as a public health problem by the MOH commenced in the 2000s. Screening of newborn babies for SCD was included in the under-five child health strategy for 2007-2011 [109]. The Ghana Health Service Programme of Work 2007-2011, advocated for the establishment of screening and management programmes for SCD in all health facilities [110]. The National SCD Control Programme was launched in 2010 with a technical committee to plan implementation. The strategic framework to guide the establishment of National SCD Control Programme was published in 2011 [4] and by 2011, a technical plan and draft policy for newborn screening for SCD was produced. The components of the Screening programme are: advocacy, education, and counselling, laboratory and operations standards, clinical management, and logistics support. The programme is currently being coordinated by the Sickle Cell Foundation of Ghana (an NGO), on behalf of the MOH.

Regarding advocacy, the National SCD Programme (NSCDC Programme) proposed the formation of a national advocacy group (Sickle Cell Disease Advisory Committee) to advocate interventions to promote awareness of $\mathrm{SCD}$, advise the $\mathrm{MOH}$ on the implementation of the programme, and facilitate the mobilisation of financial, human and technical resources to support the programme. By 2011, various organisations, groups and individuals had been invited by the $\mathrm{MOH}$, but the committee had not met as at June 2016 [111]. The advocacy group also included the Sickle Cell Association of Ghana (SCAG), with the role to create sustained political awareness of the disease. Currently, there are five SCAG branches attached to SCD clinics in four regions (Greater Accra, Ashanti, Eastern, Central and Brong Ahafo). In 2004, a National SCAG was formed, headquartered in Kumasi. As part of its advocacy role, the national SCAG organises commemoration of the African Day of SCD (10 ${ }^{\text {th }}$ May) and International SCD Day (19 $9^{\text {th }}$ June) and partners with the Regional hospitals to open SCD 
clinics and branches of the association. The local branches of the SCAG focus on providing psychosocial support, engage in blood transfusion promotion, patients focused education and general public education. Other activities include entertainment events for patients, provision of essential medicines, promotion of paternal involvement in SCD child care, and arbitration of domestic conflicts $[62,112]$. During my trip for data collection in Ghana, I learnt that almost all regional branches were under-functioning with dwindling membership and irregular meetings. The reasons cited included lack of institutional support following the completion of the NSSCD project, lack of interest from health professionals, lack of funds, weakness in organisational structure, patient fear of stigmatisation, and failure of the Association to meeting the socio-economic expectations of members.

In terms of public education, the National SCD Programme proposed patient-focused education for patients, families and the general public. The plan for education was to hold sessions in all SCD clinics. The public education plan involved public-wide distribution of information, education and communication materials such as radio and television jingles and leaflets, and partnering with the school health programme to integrate SCD education into basic school curriculum. The plan also involved health education talks to the general public targeting organised groups such as schoolchildren, religious groups, social and professional associations, NGOs and other organisations.

The only organised education and counselling service provided was part of the Newborn Screening Project from 1993 to 2008 in Kumasi. This included patient-focused education and counselling at the KATH SCD clinic for patients, and community education through churches and schools to promote awareness of the NSSCD project [54]. There has not been any other formalised education and counselling programme following the completion of the NSSCD project. I am aware that some programmes have been delivered on an ad hoc basis. It is believed that in the absence of systematic public education, many people lack relevant knowledge of the SCD resulting in uninformed decision about choosing marriage partners, and stigmatisation of the affected people and families Dennis-Antwi [54]. Currently, the socio-economic impact of lack of education and limited knowledge of SCD is unknown as no cost benefit analysis has been conducted. 
With respect to preventative services, genetic counselling and testing services were proposed by the national programme to reduce the incidence of SCD. A certification programme for genetic counsellors was developed by the SCD Foundation and training commenced in 2011 for a small number health professionals. Larger scale training has been planned for health professionals from June 2016 with health facilities required to sponsor staff [111]. Until more people are trained, professionally delivered genetic counselling and testing will remain limited. In most parts of Ghana, people marry or give birth unaware of their SCD genotypes. Many become aware of their status after giving birth to SCD child. Churches now offer genetic counselling services as pre-condition for church blessings or weddings but church counsellors often lack requisite knowledge to provide accurate interpretation or counselling $[4,54]$. This lack of professional counselling and testing poses a risks for more SCD children to be born.

Furthermore, diagnostic services for SCD such as $\mathrm{Hb}$ electrophoresis are limited to regional and the tertiary hospitals as most district and sub-district facilities only provide sickling tests which give inconclusive diagnoses. As a result, it is believed that many SCD patients are diagnosed after frequent hospital visits with related problems or die without having been diagnosed [62]. The NSCDP advocates universal new-born screening, and opportunistic screening at well baby clinics (4-6 weeks) but, to date, the only organised screening programme is the new-born screening project that has not been extended beyond original sites [111].

With respect to clinical care, the National programme proposed training of district staff in the diagnosis and treatment of SCD, establishing SCD clinics in all regional hospitals and national centres of excellence, and provision of specialised care based on CHAM. As of 2016, there were limited access to specialist, organised SCD focused clinical services. Only one specialised clinic (Korle-bu) existed from the 1960s to the early 1990s when the Kumasi clinic was established as part of new-born screening. The remaining six clinics including five public and one private hospital (Agogo Presbyterian, 37th Military Hospital, Narh-Bita Hospital-Tema, Tema General Hospital, and Sunyani Regional Hospital) were established in the 2000s. Seven new clinics were established in seven district hospitals in the Ashanti Region after 2010 [4]. An SCD centre to manage referrals is being constructed at KATH [113]. 
I am aware that some regional SCD clinics operate inconsistently. For example, two regional hospitals (Koforidua, Sunyani) only operate when there are interested physicians. The Kumasi clinic (KATH) currently offers only paediatric services as the SCD clinic was integrated into the paediatric department after completion of the NSSCD project. Adult patients attend the mainstream OPD services. The Newborn Screening Project also advocated the development of district hospitals to provide care. Limited progress has been made as only three of the seven district hospitals (Bekwai, Effiduase, Suntreso) were functioning by mid2016 [111]. I am aware that two of these clinics (Bekwai, Effiduase) are managed by nurses.

Within the public health system, clinical care for SCD is provided at all levels depending on presenting problems. Many of these facilities tend to provide penicillin prophylaxis, folic acid supplementation and malaria prophylaxis to young children. General guidelines for therapeutic care for SCD is included in the standard treatment guidelines [114]. Pneumococcal vaccination was included in the National Expanded Immunisation in 2012. The MOH Ghana [4] asserts that these public health measures, including improvement in obstetric and maternal health, have had improved morbidity and mortality outcomes.

Regarding research and surveillance, the national programme proposed basic and applied research to support comprehensive management and preventive care. Proposed areas of research included examining knowledge, behaviour and practices, barriers to access, measures to reduce stigma, impact of SCD on families, effect of chemoprophylaxis, improved diagnostic methods, clinical studies such as pain relief and management of co-morbidities. Alternate medicines such as herbal preparations and acupuncture, and psychosocial support The MOH also advocated the use of a surveillance system to collect data on SCD and related risk factors and effects on prevention and treatment. The plan included a surveillance system consisting of a database accessible at the treatment sites to ensure continuity of care. It also included development of an electronic records accessible at the maintenance of the SCD register which would be collated and managed by the Centre for Information Management [4].

The national research and surveillance plan has not been pursued. Up till now, the only published research on sickle cell is the NSSCD project. Implicit in this project was an organised data collection system. The Sickle Cell Foundation continues to collect data on 
new-born screening, inpatient and outpatient attendants at KATH. Nationally, no registry for SCD exists and existing research is usually individual students' research work, most of which remains unpublished. Hence, there is lack of official statistics required for planning and management of SCD. I am aware that a pilot Hydroxyl Urea (HU) study has recently commenced at KATH and another medicine, Prasugrel (a platelet aggregation inhibitor), is being piloted for SCD care in a mission hospital (Agogo) in the Ashanti region.

On capacity building, the NSCDP planned to conduct regular in-service for health care workers, including the basic management of SCD in the curriculum of pre-training institutions. The programme also proposed to solicit the expertise of Ghanaians in the Diaspora for material and technical support to enhance SCD care. It also advocated for training and employment of key health personnel into the SCD specialist programme. Minimum progress has been made regarding the capacity of health professionals. The only organised training for SCD management used to be part of the NSSCD project for the project staff. Since the completion of the project, there had been no formalised training until recent efforts by the SCD Foundation. The Foundation conducted regional training workshops for 551 health workers to assess their training needs in all regions in Ghana. The findings were used to develop SCD management protocol for training health professionals which was yet to commence by mid-2016 [111]. Until now, many health professionals have limited knowledge in SCD management due to limited in-service training. It has been argued that as a result of limited training and protocols for SCD management, health professionals in most parts of the country manage SCD patients with their own knowledge and experiences and SCD management in Ghana is unstandardised [62].

It can be concluded that limited achievements have been made in providing SCD focused services in Ghana, despite the establishment of SCD focused policies and programmes. Existing services are basic, mainly reactive with a focus on clinical management of presenting complaints. Interventions that are anticipatory, preventive, promotive and rehabilitative, required for comprehensive management are lacking. Furthermore, the services are paternalistic with patients the passive recipients of health services. There is the need to incorporate chronic care models including self-management in the provision of health services for people with SCD in Ghana. Consequently the findings of this study was anticipated to contribute to filling this service gap. 


\section{Chapter 3: Literature Review}

This literature review has been conducted to highlight the scholarly work on selfmanagement for SCD. The purpose of the review was to examine existing self-management related research to provide a context for situating the current study. In this chapter, I first provide a description of the search strategy. Secondly, I review self-management literature for genetic conditions (SCD, haemophilia, cystic fibroses) to identify common themes. Thirdly, I present findings of the SCD self-management research through a narrative synthesis of existing studies. Fourthly, I summarise the key variables and concepts derived from the review that were relevant for informing the subsequent stages of the study. Finally, I conclude by summarising the gaps in the current SCD self-management literature, and indicate how the current study contribute to future knowledge.

\subsection{Search Strategies}

Four main strategies were used to search the literature for the study. Firstly, electronic searches were conducted in these databases: PubMed, Cochrane Library, EBSCOHOST (CINAHL plus, health business edition, health source-consumer edition, health source, nursing academics edition), Psych Info, Web of Knowledge, Google Scholar (search engine) and other online sources. The electronic searches were sourced mainly through the Victoria University of Wellington's library system. The search focused on genetic disease including sickle cell, haemophilia, and cystic fibrosis and erythema bullosum. These conditions were chosen because people who had them could not take any actions to prevent the condition as they were born with it. These conditions were combined with self-management and related terms including self-care, self-treatment, self-help, self-medication, complementary and alternative therapies, adherence, home care and coping. The initial search term was built in PubMed using Mesh and text word searches, and was adapted for the other databases. The second strategy involved a search for similar papers from the references of papers identified through the database searching, as well as citation searching. This process continued iteratively until no new relevant materials were located. Papers were included when the focus was on patients or parents performance of the genetic diseases. There was no age group or geographical restriction to the search. Papers were excluded if they involved only health professional delivered interventions without active involvement of patients. 
More than 700 papers were produced from the electronic searches. However, the majority were concerned with basic sciences, clinical management or professionally administered CAM. The titles of all papers were reviewed and abstract read for papers that were potentially relevant. When these were removed and years limited to 2003 to 2013, human subjects, and English language, the total remaining was 65 articles. A further 4 articles were identified in 2016. No relevant studies of erythema bullosum were identified.

\subsection{Overview Self-Management Studies on Genetic Diseases}

In this section I review self-management research for SCD, cystic fibrosis and haemophilia which are genetic conditions present from birth that are lifelong. The purpose of the review was to identify self-management themes common to these conditions to further inform conceptualisation of the study. The overall results identify that self-management for these conditions is under-researched as limited published literature was located. Almost all self-management studies on SCD were from the USA and that of cystic fibrosis were from Europe and Haemophilia were from Europe and the USA. The studies were thematically grouped into four areas.

One group of studies, mostly non-experimental, explored various aspects of selfmanagement practices among patients and caregivers. Common areas were pain relief, use of recreational drugs, life stories, tracking and recording, use of complementary and alternative medicines and psychosocial coping [17, 115-130]. The second and largest group of studies were interventional; they required patients to perform self-management actions prescribed by the researchers and requested participants to assess and record aspects of disease or outcome, or self-administer therapeutic regimens usually administered by health professionals. The outcome measures in these studies involved adherence, compliance or effectiveness of the self-administered regimen [131-138]. In the majority of these studies the focus was to test the feasibility of a prescribe regimen by health professionals among patients and parents [31, 132, 139-144]. A small collection of interventional studies also involved a comparison between patients' performance of self-administered regimens to health professionals delivered regimens $[136,145,146]$. The third group of studies evaluated patients and caregivers' perceptions regarding self-management they practices [147-153]. The fourth group focused on testing theories of self-management and associated factors [118, 122, 134, 154-158]. 
The common themes derived from the studies were self-monitoring, self-diagnosis, self-treatment including use complementary and alternative medicines, psychological coping and self-management support.

\subsubsection{Self-monitoring and self-diagnoses}

Studies on self-monitoring were the most common that involved assessment and recording of data. This included self-assessment of various aspects of disease, treatment or outcome, recording of findings for assessment, and self-administered treatment or hospital utilisation. Aspects of the diseases that patients and carers recorded were pain and related symptoms for SCD [116, 122, 131, 140], self-administered oxygen administration, spirometry and use of nebulisers for cystic fibrosis [136, 141], and self-infusion of clotting factors for haemophilia [130, 138, 153].

The use of diaries or journals (paper and electronic) were common for monitoring and data recording. Paper based diaries were recorded and submitted periodically to a collation site usually through the post [116, 131]. Modified forms of diaries such as colour coded diaries were used for paediatric patients [116]. The electronic based diaries were hand-help computers, smart phones or computers connected to the internet.

A number of studies examined the feasibility of electronic diaries or compared the effectiveness between paper and electronic diaries. The evidence from these studies identified electronic diaries are more advantageous than paper-based diaries. They were more user friendly and accurate than paper-based diaries [130] and ensured better compliance to prescribed programmes and recording schedules [138]. Use lead to prompt communication between health workers and clients, resulting in better monitoring and prompt management of the diseases and reduction in complications [130-132, 140, 141, 153]. Some researchers have recommended that electronic diary systems can adequately replace paper-based systems for self-monitoring and recording purposes [141, 153].

\subsubsection{Self treatment}

Self-treatment either involved self-administration of medicines or prescribed therapeutic regimen. One cluster of these studies were non-interventional and investigated existing practices among patients and parents [17, 123, 125, 159, 160]. A second cluster, 
which were interventional, assessed adherence to recommended treatment practices, tested feasibility of patients' self-administration of treatments that were previously administered by health professionals or compared patients' self-treatment with professionally delivered treatments on same therapies [145, 159].

Among studies on SCD, self-treatment mostly involved use of analgesic medication and CAM $[125,131]$. The use of analgesics was more common among SCD patients [125, 131], and haemophiliacs [160] but not with patients with cystic fibrosis. Among haemophiliacs, self-infusion of blood clotting factors was the focus of most studies. Studies that investigated feasibility showed that patients and families could successfully infuse the clotting factors at home [138, 144, 152, 159]. A study by Balaguer and Gonzalez de Dios [145] demonstrated that patients could self-administer intravenous antibiotics. The study compared self-administered intravenous infusion at home to inpatient-care in the hospital and found no significant difference between the two methods. In other studies, patients successfully administered supplemental oxygen and nebulisers at home with results that were similar to hospital setting [126, 161].

A small collection of studies explored the use of alcohol and illicit drugs for selftreatment and effects in SCD and haemophilia. Substance use was related to pain control among patients with SCD and haemophiliacs. Alcohol and cannabis were two substances identified [117, 119]. Findings reported that 31\% of SCD patients had used alcohol [119], and $36 \%$ had used cannabis [117]. One fifth of haemophiliacs also reported using alcohol or cannabis for pain control [160].

Levenson et al. [119] reported advantages of alcohol users had over non-users including increased pain relief from opioids, decreased emergency visits to hospitals, and overall better summary score on QOL scale. In addition, alcohol users described better pain coping capacities than non-users. However, Elander and Barry [160] did not identify any significant difference between users and non-users of cannabis for severity of pain or hospital visits despite sedation and relaxation effects reported by SCD patients. In summary, these researchers found limited conclusive evidence on the effects of alcohol, cannabis and other substances in the management of pain. There are plans to conduct larger studies to elicit the effect of cannabis use among SCD patients [162]. 
Several studies investigated the use of CAM approaches for self-treatment. The reporting of CAM for self-treatment was identified in SCD and cystic fibrosis but not in haemophilia. One study reported that homeopathy administered by practitioners, had the potential for reducing the frequency of bleeding in haemophilia [163]. Tanase and Zanni [124] reported that self-administered CAM was used by $77 \%$ of 97 cystic fibrosis patients and Dampier [116] reported $84.6 \%$ use amongst 37 SCD patients. The use of CAM was more common among caregivers for paediatric and young children with SCD and cystic fibrosis $[116,121]$. The commonest CAM therapies were bioenergy and lifestyle/body mind therapies, prayers and spirituality $[121,123,124]$. Findings showed that CAM approaches were mostly used concomitantly with other conventional treatment approaches [121, 123, 124]. In terms of benefits derived from the use of CAM, the findings show that patients and caregivers generally perceived CAM use as helpful. Given that most CAM related studies were crosssectional and based on patients self-reporting, researchers expressed the need for research in the form of multicentre trials and large scale studies to assess the applicability of CAM use in self-management [121, 139].

In addition to the use of medicines, studies revealed other mechanical therapies such as exercise, spirometry and massage for self-treatment. Researchers reported adherence to unsupervised exercise prescription at home (based on patient activity assessment) resulted in increased exercise behaviour, improvement in lung function and QOL of cystic fibrosis patients [142]. Among haemophiliacs, the use of exercise is reported to improve physical and psychosocial status of sufferers. Specific benefits include improved balance, and decreased destruction of joints [164]. In a systematic review of the benefits of exercise Hill, Fearn [165] concluded that the benefits of exercise are maximised when patients commence clotting factor prophylaxis before three years of age. Grzincich et al. [141] concluded that patients could adequately perform spirometry at home and this intervention decreased the workload in the cystic fibrosis clinics. Among SCD patients, one RCT found that massage therapy was useful in reducing depression, anxiety, pain and functional status but did not change hospital utilisation [139].

Most researchers described the interventions used by patients and carers as safe and effective for self-treatment and requested their integration into patient's self-management interventions. However, a few researchers recommended further trials should be conducted to 
provide more evidence for the use of these measures. In short, self-treatment is common among the three conditions, and involves the use of medicines (conventional, CAM, alcohol, cannabis) and non-medicines involving mechanical interventions.

\subsubsection{Coping strategies}

The review highlighted strategies people use to cope with their conditions and factors that affect that can affect individuals' coping. These strategies emanate from the individual, immediate family and health services. The commonest individual coping strategy reported by the researchers involved the use of religiosity [123, 124, 129, 166, 167]. Religious tenants that encourage hope, comfort and wishful thinking were found to have positive coping outcomes for patients and families $[17,123,129,166]$. However, negative religious tenants in the form of vicarious despair and pleading were found to be associated with adverse physiological measures including deterioration of lung function among cystic fibrosis patients [166]. Negative religious coping effects were predictive of adverse child mental health, parent anxiety and adverse parental emotions [129]. No study reported a positive relationship between religion and positive physiological outcomes for these conditions.

Another individual coping strategy reported related to the use of self-concepts such as patient or parental acceptance of the condition, level of autonomy, and confidence. Atkins and Walters [168] concluded that parental coping is enhanced by acceptance of their child's situation and recognition of parental responsibility to care for the child. Furthermore, autonomy and self-efficacy were found to positively influence self-management of cystic fibrosis [140, 169]. A higher level of parental autonomy for example, was found to lead to greater autonomy, and confidence in self-management behaviours among adolescence with cystic fibrosis [169].

Individual strategies further included patients self-esteem, intentional or purposeful forgetting and perceived benefits of the self-management action. These factors were found to improve patient's adherence to prescribed self-management regimens [148, 170]. Patient's level of self-esteem was found to influence psychological adjustment. One study [170] found that haemophiliacs generally have good adjustment to their condition except with self-esteem in relation to the penile function. This emotional strain and worry concerned future marital 
relationships [167]. The researchers concluded that self-esteem is the main determinant for the haemophiliac to adjust to their condition or develop depression.

Regarding strategies emanating from the patient's immediate family, research linked positive self-management practices to factors such as social support [148], and family climate such as the level of conflict and disengagement in family [137]. In relation to health services strategies, studies have shown that availability, accessibility, and health professionals' attitudes influence adjustment [17, 123]. For example, Fiese and Everhart [137] found that accessibility of professional support delivered by sympathetic and competent professionals enabled parents to adjust and come to terms with their child's condition. The next section focuses on SCD self-management research.

\subsection{Research on Self-Management for Sickle Cell Disease}

This section presents a detailed review of international literature on SCD selfmanagement. A total of 20 publications focused on SCD self-management. These included 18 empirical studies including 13 quantitative $[116,117,119,121,122,125,131,132,139,147$, $156,171,172]$ and five qualitative studies $[17,118,123,173,174]$. One publication provided a theoretical framework [175] and another was an editorial [176]. Most of the empirical studies recruited adult populations $(n=9)$, adolescents or children $(n=7)$, and caretakers $(n=3)$. Sixteen studies recruited participants from SCD clinics and three recruited from both clinic and community. Studies that recruited from the community mainly accessed the participants from SCD networks such as associations and foundations. Studies were from the USA $(n=16)$, UK $(n=1)$, Jamaica $(n=1)$, and Brazil $(n=1)$. No studies were located in Ghana. The literature is organised into quantitative and qualitative sub-sections. Concepts and variables were derived from the studies to guide the subsequent stages of the research.

\subsubsection{Quantitative Studies}

Three of the 13 quantitative studies [121, 147, 171] were cross-sectional and the remaining were longitudinal. The aims of the quantitative research were either to understand pain frequency, characteristics, or related symptoms, and home management, pain selfmanagement interventions, or test feasibility of pain management technology. The common themes from the studies were self-monitoring, self-diagnosis and self-treatment. 


\subsubsection{Self-monitoring/diagnosis}

Six quantitative studies involved patients' self-monitoring [116, 119, 122, 131, 132, 139]. Patients' self-monitored pain, associated symptoms, home treatment and frequency of hospital utilisation as a result of pain experiences were explored. Almost all studies used similar methods requesting patients to self-measure their pain using assessment tools, interpret their pain experience, identify related symptoms, and record their findings into paper or electronic diaries. The USA-based prospective cohort study by Smith et al. [122] sought to establish the prevalence of self-reported pain and the relationship of pain crises and hospital utilisation among adults with SCD. The participants were recruited from SCD associations, clinics, emergency departments (ED), referrals from other health departments, and direct recruitment from health fairs and radio announcements. Participants completed a daily diary for 6 months, recording their maximum pain, whether they were in a crisis; medication taken for pain; and unscheduled physician visit, ED presentation or hospitalisation

Several studies $[116,122,131]$ investigated self-monitoring conducted simultaneously with elements of self-diagnosis. The researchers asked patients to self-identify, define and categories their pain and related symptoms to pre-determined categories. The researchers typically asked patients to determine their pain intensity, location and quality, the type of pain, and associated physical and psychological impacts. Pain intensity was measured quantitatively with numerical pain scales or qualitatively using descriptive terms such as mild, moderate or severe pain or highest and lowest pain [121, 131, 139]. In Jacob et al [131] study, the patients were also asked to assess the quality of their pain by choosing from 67 pain descriptive words. Patients were asked to determine whether their pain constituted a crisis or non-crisis $[116,119]$. In other studies, patients were asked to identify associated symptoms and impact of their pain. Two studies $[122,131]$ required patients to determine physical symptoms using symptoms checklist with terms including tiredness, fatigue, headache, jaundice, and respiratory distress. In addition, studies investigated the psychological impact of pain on thoughts and feelings and distress and sleep quality $[117,131]$. Howard et al. further investigated the impact of pain affecting work, school, households and friends.

Self-monitoring and diagnoses were facilitated by e-health technologies in two studies [131, 132]. Typically, patients' measurements were recorded into electronic devices and 
transmitted to the researchers. In one study [131] there was live communication between the patient and an advanced practice nurse (APRN), specialised in SCD who remotely monitored patients' entries and contacted patients who required attention. Patients and the APRN communicated through telephone and text messages giving prompt recommendations to patients. The researchers concluded that remote monitoring facilitated prompt pain and symptom management averting negative impacts of SCD. McClellan et al. [132] also conducted an interventional study among 19 patients (9-20 years) to evaluate the implementation of pain protocol with SCD using handheld wireless devices. The participants were given a device loaded with a pain diary and audio files to guide practise of cognitive behaviour therapy. They answered questions regarding pain twice daily and transmitted the diaries to the researchers. The researchers concluded the e-diary approach ensured prompt communication between health workers and patients, facilitated prompt management and reduced the complications associated with $\mathrm{SCD}$.

Overall, the researchers using quantitative methods involving self-monitoring and diagnosis expressed positive impressions about patients monitoring and diagnosis. Patients' diary completion ranged from $61 \%$ to $90 \%[116,122]$. The patients self-monitoring and diagnosis activities enabled the researchers to understand the incidence of SCD pain, related symptoms and trigger factors. For example, Jacob et al. [131] reported that $42.6 \%$ of pain occurred in the morning whilst $57.4 \%$ occurred in the evening. The severity of pain were distributed as mild (44.4\%), moderate (41.8\%) and severe (13.8\%). The most frequently affected areas were lower back (25.8\%), chest and abdomen (22.5\%), back (22.4\%), front (20.3\%) and knees (16.5\%). Moreover, patients monitoring and diagnostic activities enable the researcher to describe the proportion of SCD painful events that are managed at home. Smith et al [122] reported patients experiencing pain on $54.5 \%$ of days and almost one third had pain daily. On $38.3 \%$ of the pain days, patients did not describe their pain as a crisis and did not seek health care. On most crisis days, many patients managed their condition at home, only making unscheduled visits to health facilities on $3.5 \%$ of these days. When scheduled visits were included, health facility utilisation rose to $5.1 \%$ of days. The researchers concluded that pain is more prevalent and severe than reported in previous studies, is mostly managed at home and, therefore, the prevalence is underestimated by health practitioners, resulting in misclassification, distorted communication, and under treatment. 
Furthermore, the studies that involved use of e-health technological devices reported higher rates of monitoring and increased patient satisfaction [131, 132]. Consequently, Jacob et al. [131] for example concluded that data transfer has potential to become a practical method to improve symptom monitoring and communication between patients and providers. Overall, there was a consensus among the interventional studies that patients and carers can be supported to effectively monitor their disease, identify problems and initiate appropriate interventions.

\subsubsection{Self-treatment}

A number of the studies involved a variety of patients' self-treatment. Almost all of these concerned pain treatment. The measures used included self-administration of therapies that were previously delivered by health professionals or patients' self-directed therapies. The commonest therapies involved use of CAM or non-pharmacological therapies, analgesic medications, recreational drugs, and psychological strategies.

CAM was the most common self-treatment strategy. Various terminologies were used to describe CAM: non-pharmacological strategies [121], complementary therapies [125], physical and cognitive behaviour activities, and cognitive behaviour coping skills [132]. Whilst one study [121] investigated only CAM therapies, three other studies investigated CAM and other therapies used by patients [116, 125, 132]. The definition and composition of CAM differed among the studies but it generally implied "unconventional" measures employed by patients and families to manage SCD at home. Sibinga et al. [121] provided the most comprehensive classification of CAM used by SCD patients. These include therapies that are bioenergetics (acupuncture, therapeutic touch/energy healing, spiritual healing, homeopathy, prayer); lifestyle/mind-body focused (relaxation techniques, imagery, hypnosis, biofeedback, self-help groups, yoga/tai chi, commercial weight loss, diet, exercise); biochemical (herbal medicine, megavitamin therapy, folk remedies); and biomechanical (massage therapy, chiropractic). Sibinga et al. further classified CAM into pharmacological and non-pharmacological whereas Dampier et al. [116] also classified CAM as passive (distraction, and talking to friends) and active (relaxation, self-hypnosis, heat, massage). Of note is that all CAM strategies reported in the research fell within Sibinga, Shindell [121] categorisation. 
All the CAM related studies involved only paediatric and young SCD patients or carers. These studies tended to conclude that CAM use is common among this population. Sibinga et al. [121] study for example surveyed CAM use among caregivers of SCD children. The results showed that $54 \%$ of the 57 caregivers used CAM therapies for their children. Of these, $42 \%$ used bioenergetics therapies (prayer, spiritual, energy healing), $28 \%$ used lifestyle/mind-body CAM (relaxation techniques, exercise, imagery, diet), 12\% used biochemical therapies (herbal medicines, megavitamins, folk remedies), and 5\% used biomechanical therapy (massage). Yoon and Black [125] also conducted a cross sectional study at a USA-based SCD clinic to explored pharmacologic and complementary therapies used for pain management by 63 caregivers of children (aged 0-21) with SCD, the prevalence and types of CAM used for this pain management, and parents interest in using complementary therapies in the future. The findings showed that more than $70 \%$ of caregivers were using some form of CAM.

Overall, the findings from the CAM studies indicated use in combination with conventional analgesics. For example Dampier et al. [116] reported physical and cognitivebehavioural activities were used on $84.6 \%$ of days with SCD pain, with analgesic medication concomitantly used on virtually all (2000 days) of these days. Analgesic only treatment was used on $10.9 \%$ of days with SCD pain, while physical and cognitive-behavioural therapy only treatment were used on $8.8 \%$ days, and neither was used on only $14.5 \%$ days. Yoon and Black [125] concluded that caregivers of children who used greater volumes of conventional pain medication were more likely to use complementary therapies. Further, findings emerging from these studies indicated that the use of CAM tends to increase with analgesic use and with increased pain intensity $[116,125]$, with multiple forms of CAM use. Dampier et al. [116] found that a single CAM technique was used on approximately $20 \%$ of the days while two measures were used $44 \%$ of days. The common types of CAM used include the bioenergy and lifestyle/body mind approaches such as prayer, relaxation techniques and spiritual activities Sibinga et al [121] and massage therapy [121, 139]. Specifically both Sibinga et al. [121] and Yoon and Black [125] found prayer and spiritual healing as the commonest CAM strategy used.

There is a consensus among the researchers that patients find CAM useful. Sibinga et al [121] reported most respondents (83\%) felt that CAM helped them and Lemanek et al. 
[139] found that youth who used massage therapy had higher levels of functional status, and lower levels of depression, anxiety, and pain than their unexposed counterparts but health service utilisation rates did not differ. As most studies were conducted in single centres using patient reports only, the researchers have recommended more rigorous, multicentre interventional studies are required.

\subsubsection{Self-medication with conventional medicines}

Apart from CAM, the research findings indicate that the use of analgesic medications is common amongst people with SCD. Non-steroidal anti-inflammatory drugs (NSAIDS) and acetaminophen were the commonest. Yoon and Black [125] found that most frequently used pain medications were ibuprofen $(37.5 \%)$, paracetamol with codeine $(32.1 \%)$ and paracetamol with oxycodone (14.5\%). However, their study did not indicate whether these medications were based on prescriptions from health professionals or purchased over-the-counter. A USAbased study by Johnson [147] involving 40 adults SCD patients found most prefer to manage their own analgesic medication, rather than a nurse, because it provides control over pain relief, better accessibility to analgesia, quick relief and independence from hospital staff.

\subsubsection{Use of alcohol and cannabis}

As indicated in section 3.0, two studies reported use of recreational drugs including alcohol and cannabis (marijuana, Indian hemp) to self-management SCD pains. The drug users were reported to have better pain relief from opioids, significantly fewer emergency department presentations due to pain, better pain tolerance and superior quality of life than non-users. In a prospective cohort study to determine the prevalence of alcohol use among SCD patients Levenson et al. [119] sampled 308 SCD adults (16 years and older) from clinical and community settings in the USA. The results identified that $31.4 \%$ used alcohol. The only difference reported between alcohol users and non-users was that alcohol users reported more pain relief from opioids than non-users, and they had fewer unscheduled ED visits. Quality of life was similar between both groups, except that alcohol users unexpectedly had better overall physical summary scores. Alcohol users were more likely to report coping by ignoring pain, and diverting attention. In a related study by Howard et al. [117] to determine cannabis use, the researchers followed 86 adult SCD outpatients from a hospital in the UK over six months. The results showed that $31(36 \%)$ had used cannabis in the previous 
12 months to relieve pain associated with SCD and the main route was inhalation. Major reasons for use were to reduce pain (52\%), and reduce anxiety and induce relaxation (39\%). There was no significant difference between severity of pain among users and non-users. The researchers concluded that cannabis use was high among study participants and may be beneficial to SCD patients for whom treatment is inadequate.

These quantitative studies provide important insights into several dimensions of selfmanagement. The findings involving self-monitoring and diagnosis reveal that pain and related symptoms are potential indicators for SCD patients self-monitoring and diagnosis. The CAM related studies also provide an understanding of unconventional medicines and nonpharmacological strategies people might use for SCD care. The quantitative research reported analgesics as the commonest medications SCD patients use for self-treatment. The two studies on alcohol and cannabis identify the role of recreational drugs for self-management. Several of these variables were investigated in the current study.

\subsubsection{Qualitative studies}

The five SCD self-management related qualitative studies included those that specifically investigated self-management and those that indirectly elicited self-management as part of exploring peoples' lived experiences with SCD. Two studies specifically investigated self-management practices [123] and recommendations [17] among adult patients in the USA. Tanabe et al. [123] grounded theory study involved seven SCD patients and a social worker. The researchers investigated the specific measures persons with SCD use to achieve optimum health "A Best Self-management Practices Workshop." was held in conjunction with the SCD Association of America that included a panel discussion by adults recruited at the annual general meeting of SCD Providers Networks and Associations. The workshop was divided into three topics: CAM, psychosocial issues, and work/education/training. The findings revealed eight themes including: self-awareness, emotional support, carer selection and success factors, nutrition, advocacy, knowledge, physical, and CAM medicine. Ballas [176], in an editorial, titled Self-Management for SCD A New Frontier categorised Tanabe et al. [123] findings as body (self-awareness, mind) social support and spirit (religiosity and spiritualisms) mechanisms. 
The Jenerette et al. [17] study investigated self-care recommendations from patients' perspectives. The researchers used descriptive qualitative methods to study the self-care recommendations of 11 middle-aged and older adults ( 6 women, 5 men) being treated at a SCD programme. The results were categorised as physiological, psychological and providerrelated self-care recommendations. Physiological recommendations related to taking care of the physical body including staying warm, staying hydrated, getting enough rest, and eating "good" food. It was recommended that individuals with SCD should avoid smoking, drinking alcohol, and using drugs and know the “do's and don'ts”. Psychological recommendations involved obtaining knowledge of the disease, listening to and learning about the body, saying prayers, and having social support in the form of someone to talk with. The option of praying and talking to someone were only discussed by females. Participants also thought that an important aspect of self-care is having knowledgeable health providers and following their advice.

Four other studies investigated the lived experiences with SCD from patients' perspectives using various qualitative strategies. Jenerette and Lauderdale [118] explored the life stories of 12 adult patients ( 7 women, 5 men) to ascertain how they explain their longevity and how they see SCD in their lives. Reasons cited for longevity included self-care (having a better understanding of the body) and learning how to take care of themselves. One aspect of this was learning to listen to their bodies and using this understanding to engage in activities that contributed to a reduction in the impact of the disease on their bodies. A key factor mentioned was having supportive family and friends for physical and emotional support and a higher power which they describe as "stubbornness" was another reason for their longevity. In addition, participants spoke of using medical care, engaging in work and education that gave them financial independence.

Similarly, a grounded theory study involving 17 SCD patients aged 18-49 years elicited the experiences of illness among individuals with SCD and their self-care strategies in Brazil Cordeiro et al. [177] The findings were reported through four categories. The first category was "building an explanation to understand the disease". In this category, the researchers explained that patients make efforts to understand SCD. The second category was called "observing time and physical signs". The researchers explained that patients measure time by counting the number of crises, isolate their body, and observe alert signs of the body. 
The third category was "making mistakes and learning self-care." The researchers asserted that this involves patients building standards for self-care from experiences of falling sick, developing specific skills for self-care, maintain an adequate diet, and attending regular medical visits. The fourth category "taking care of faith and spirituality" includes seeking strength to understand suffering, having faith and hope, and believing in a healing miracle.

A similar study from Jamaica [173] explored the lived experiences of adolescents with SCD using descriptive qualitative design. In-depth interviews were conducted with six adolescents with SCD. Major themes identified were psychosocial challenges including fear of death, absenteeism from school, decreased physical activities, and sexual intercourse challenges. The participants highlighted the key factors that helped them to live with their disease: positive self-concept and increased social support. The most significant of these was social support. This included support they received from family, school, and peers which made them feel accepted. The participants also mentioned various coping strategies they utilised to address challenges of the disease including praying, watching television, and surfing the Internet. Thus, although the study was restricted to adolescents (8-19yrs) it identified the importance of psychosocial concerns and coping strategies.

A cross sectional study by While and Mullen [171] in the UK also explored the lived experiences of 11 young SCD patients (12-16 years) as they transferred to adult services. This study used both qualitative and quantitative approaches. The findings showed a number of areas that boarded on self-management. These were concerned with use of prophylaxis, identification of trigger factors, actions taken during painful crisis, and social support. The prophylaxis taken were penicillin and fluids. The participants reported variable adherence to use of penicillin prophylaxis and some discussed their challenges in adhering to the prophylaxis. The trigger factors named by the participants were exercises (running, swimming, football, play, fighting), temperature (being cold or wet, sudden change), hydration (not enough drinking, sweating), emotional state (lots of stress, upset and excitement). Regarding social support, the participants mentioned a range of individuals they felt comfortable talking to including parents, siblings, health professionals, religious leaders and teachers. Many said that they receive the support during painful crisis but others did not. The researchers concluded that young people with SCD require social support drawing on a range of expertise and that the medical model of health delivery may not meet their need. 
Examination of the results of the qualitative research revealed five background themes reflecting self-management strategies. Four themes (self-monitoring, preventive health, selftreatment, knowledge acquisition) concerned strategies used to management physical health whereas one (coping strategies for psychological well-being) concerned psychological health. The most common of these themes were self-monitoring and coping strategies for psychological well-being.

\subsubsection{Self-monitoring}

According to the results of the qualitative studies reviewed, self-monitoring is the commonest self-management strategy mentioned by the participants. Self-monitoring was conceptualised similarly across the studies using different terminologies typically portrayed as patients being in tune with their physical body, and listening to or observing physical sensations or cues. It also involves identifying and managing triggers of disease exacerbation. Different studies used different phrases to denote self-monitoring, including: self-awareness [118, 123], listening to the body [118], and listening and learning about the body [17]. In two studies, self-awareness or bodily awareness was found to be the commonest strategy used among adult SCD patient $[118,123]$. However, these two studies categorised self-awareness differently. Tanabe et al. [123] categorised self-monitoring as a physical strategy whereas, Jenerette and Brewer [118] categorised it as psychological self-management strategy. According to Tanabe et al., self-awareness referred to patients' awareness of behaviours that diminish or promote their health and factors that triggers exacerbation of their disease. On the other hand Jenerette and Lauderdale [17] identified that participants recommended the most important psychological self-care recommendation was how to listen to their bodies, they used phrases such as "listening to what your body is telling you".

Often, self-awareness was described as observing individual limits or threshold and adjustment strategies. For instance, Jenerette and Lauderdale [118] reported patients' knowledge of their limits and triggers of crises as key features for self-awareness. Selfmonitoring was also viewed as observing time and counting signs. Patients tended to monitor the time in the season when they had painful crisis, and remissions from crises. The patients also observed alert signs of the body and common triggers as mentioned by While and Mullen [171]. 


\subsubsection{Preventive health and maintenance}

It was clear from the findings of the studies that patients employ strategies to prevent disease exacerbation. These strategies tended to target factors that can directly or indirectly exacerbate their disease. The direct strategies involved avoidance of known (by patients) precipitating factors for painful crisis such as such as cold weather, and exhaustive activities. The indirect involved healthy behaviours that promote homeostatic balance such as nutrition, hydration and supportive medication. Similar areas of preventive health actions were reported across the studies but the extent differed. The majority of the reports concerned food, temperature and hydration. Jenerette et al. [17] reported the most comprehensive preventive health strategies, including: staying warm, staying hydrated, getting enough rest, and eating "good" food, avoid smoking, alcohol drinking and other hard drugs, knowing the "do's and don'ts". Cordeiro et al. 177] findings elicited preventive health among patients as "learning to isolate the body". This implied patients' isolation of their bodies during winter and maintaining an adequate diet. Similarly in Tanabe et al. [123], the participants mentioned the need to eat a healthy diet, consisting of fruits, vegetables, and unrefined food, vitamins and herbal supplements. Forrester's [173] study also reported actions participants undertook to avoid crisis and complications as social activity restrictions, having specific way of dressing, adhering to medical and dietary regimen.

Another aspect of preventive health reported by two studies $[123,171]$ concerned the choice of occupation that are compatible with SCD. The participants in Tanabe et al. [123] recommended strategies for successful career selection. These included job that are less physically demanding in a conductive environment. The participants' advise SCD patients to consider the weather of the workplace, the environment of the geographical area, and careers that give them some flexibilities.

Use of prophylaxis was another preventive health strategy reported. While and Mullen [171] identified use of penicillin prophylaxis and frequent oral fluids intake as common prophylaxis. The participants in the study reported variable adherence to use of penicillin prophylaxis. Another aspect of preventive health $[123,171]$ concerned the choice of occupation that is compatible with SCD. The participants in Tanabe et al. [123] recommended strategies for successful career selection that included jobs that are less physically demanding 
in an environment compactible with SCD. The participants' advice to SCD patients to consider the climate of the workplace, the environment of the geographical area, and careers that provide flexibility.

\subsubsection{Self-treatment}

The study findings identified limited information on patients' self-treatment strategies. Although three studies [123, 171, 173] reported findings related to self-treatment, only While and Mullen [171] provided specific actions. While and Mullen found that during painful crises, the participants reported taking analgesics (paracetamol, ibuprofen, codeine), nonpharmacological therapies (deep breathing, deep heat, rubbing, watching TV, rest and sleep, lying in the dark room, squatting) and other prescribed medications. Tanabe et al. [123] reported use of CAM among the participants but all the strategies mentioned apart from massage appeared professionally delivered. Forrester et al. [173] also reported that people treated themselves at home to avoid hospitalisation as hospitalisations prevent them from engaging in social activities but no specific strategies were mentioned.

\subsubsection{Knowledge acquisition}

Two categories of knowledge were reported across the studies regarding understanding the hard facts of SCD and knowledge of self-care. Whilst only one study [123] recommended knowledge acquisition on hard facts of SCD as important, the majority of the findings related to knowledge of self-care. In the Tanabe et al. study, participants' recommended SCD patients should be aware of their baseline laboratory values such as haematocrit and $\mathrm{Hb}$. The participants recommended SCD patients cultivate the ability to accurately describe their medical history and complications. Other self-care knowledge concerned patient awareness of laws and policies protecting their employment rights. Some studies reported how patients had cultivated self-care skills. The findings identified that selfcare skills tend to be learnt from experience and reading. Jenerette and Lauderdale [118] found that SCD children learned the principles of self-care from parents and in adults learnt through personal experiences. Jenerette and Brewer 17] reported participants also learnt more about SCD from the internet. 


\subsubsection{Coping strategy for psychological well-being}

Psychological coping was another strategy commonly reported by the qualitative researchers that was enhanced through patients' intra-personal and external resources. Intrapersonal strategies reported by most studies involved the use of faith, prayer and spirituality, diversionary strategies, and positive self-concepts.

The use of faith prayer and spirituality was reported as means of coping with daily SCD related challenges, and as a strategy for therapeutic care during illness in three studies $[118,123,173]$. According to Tanabe et al. [123], spiritual support was the second most common self-management strategy after self-awareness. In two studies $[17,118]$, the participants all of whom were Christian, frequently prayed for God to give insights to clinicians to accurately diagnose their problems and offer appropriate treatment. Prayer was also used to manage anxiety during emergency room visits. Forrester et al. [173] reported similar findings that SCD patients used prayer, fasting and spirituality as a major coping strategy during the periods of disease exacerbation.

Another strategy reported were diversionary strategies aimed to distract patients' attention from problems and were often associated with time of experiencing illness. Jenerette et al. [155] reported that patients used distraction strategies such as praying, watching television, and surfing the Internet. Other intra-personal strategy revealed by the researchers' concerned positive self-concepts. Jenerette et al. [17] reported patients having confidence as a key strategy for self-care. Another self-concept was the ability to work and generate income. Similarly, While and Mullen [171] identified participants who reported having positive selfperceptions supported their ability to control SCD rather than the disease controlling them. Studies also reported external factors that affected patients' ability to cope SCD. Emotional support derived from families, friends, health professionals, teachers (children) and religious leaders was common. Patients who reported having been treated with support and understanding reported experiencing significant comfort [118, 171, 173].

To summarise, the qualitative research reported several dimensions of patients' selfmanagement from participants' perspectives. Unlike the quantitative studies that usually investigated specific self-management actions when patients experienced pain or related symptoms, the qualitative researchers' elicited strategies patients use when they experience 
both stable conditions and illnesses. The research revealed patients' self-monitoring strategies, medicinal and non-medicinal strategies and psychological strategies used during stable conditions and manage illness and also used to promote health.

\subsection{Conclusion}

Current research has begun to capture a range of variables and concepts that have enhanced understanding of SCD self-management. Several innovate research approaches have been used to study SCD self-management including quantitative and qualitative, interventional and non-interventional studies, longitudinal and cross-sectional studies, empirical and theoretical studies. Some researchers have also taken advantage of modern technologies to enhance patients' self-management through use of e-health systems. Diverse participants including patients and parents, adults and children have been studied by the researchers. The researchers have samples mostly from SCD clinics whilst others have sampled from communities. Various dimensions of self-management were derived from the research including self-management actions and factors that influence self-management regarding SCD (Table 5).

Five main gaps exist in the current literature. Firstly, there is paucity of published literature for SCD self-management in low and middle income countries including Ghana. Almost all the reviewed studies were conducted in the USA. Hence, there is limited understanding of cross-cultural dimensions of self-management for SCD. Secondly, almost all SCD studies were centred on pain with limited consideration given to other problems and complications. Thirdly, most quantitative studies were focused on single components of selfmanagement, usually pain monitoring and management, tended to ask participants to choose from closed questions which tend to favour highly developed healthcare systems. Fourthly, most qualitative studies were concerned with coping or lived experiences. These studies, which tended to be adult-focused specifically focused on self-management from patient's perspectives. Furthermore there have been limited application of mixed method research and most studies involved participants who could read and write, able to use technologicallybased devices and engaged with SCD clinics. 
Table 5. Summary of variables and concepts derived from literature review

\begin{tabular}{|c|c|}
\hline Category & Variables/ Concepts \\
\hline Aspects of investigated & $\begin{array}{l}\text { Pain, crisis, pain associated symptoms (tiredness, fatigue, } \\
\text { headache, jaundice, respiratory symptoms), pain triggers factors, } \\
\text { psychological outcomes (levels of anxiety, depression), and } \\
\text { fatigue }\end{array}$ \\
\hline Trigger factors & $\begin{array}{l}\text { Exercises (running, swimming, football, play, fighting), } \\
\text { temperature (being cold, being wet, sudden change), Hydration } \\
\text { (status: not enough drinking, sweating), emotional state (lots of } \\
\text { stress, stress, upset, excitement). }\end{array}$ \\
\hline Preventive health & $\begin{array}{l}\text { Career selection, nutrition, staying warm, hydrated, rest, good } \\
\text { food, avoid smoking, alcohol, hard drugs, knowing do's and } \\
\text { don'ts, Adequate diet, isolating the body, protecting and avoiding } \\
\text { precipitating factors, adopting changes in eating habit, using } \\
\text { vitamins }\end{array}$ \\
\hline Self-monitoring/ diagnosis & $\begin{array}{l}\text { Pain assessment (differentiating between crisis and non-crisis } \\
\text { pain), self-awareness (listening to the body), learning about the } \\
\text { body, journaling or use of diary, observing physical signs, } \\
\text { observing time and counting the number or crisis, observing alert } \\
\text { signs of the body functions, learning self-care, observing triggers }\end{array}$ \\
\hline $\begin{array}{l}\text { Self-Treatment - Self- } \\
\text { administration with } \\
\text { conventional medicines }\end{array}$ & $\begin{array}{l}\text { Prescribed or non-prescribed. Common analgesics (NSAIDS), } \\
\text { paracetamol, paracetamol with codeine, paracetamol with } \\
\text { oxycodone }\end{array}$ \\
\hline $\begin{array}{l}\text { Self-Treatment - Non- } \\
\text { pharmacological pain } \\
\text { strategies }\end{array}$ & $\begin{array}{l}\text { Deep breathing, warm baths, relaxation exercises, sleep/nap, } \\
\text { watching TV, reading, hot bath /heating pads, massage, talking } \\
\text { with people, praying, and relaxation, meditation and self- } \\
\text { hypnosis, lying in a dark room. }\end{array}$ \\
\hline $\begin{array}{l}\text { Self-Treatment - CAM } \\
\text { therapies }\end{array}$ & $\begin{array}{l}\text { Prayer, spirituality, energy healing, relaxation techniques, } \\
\text { exercise, imagery, diet, herbal medicines, megavitamins, folk } \\
\text { remedies, massage, muscle relaxation, guided imagery }\end{array}$ \\
\hline $\begin{array}{l}\text { Self-Treatment - Use of } \\
\text { recreational drugs }\end{array}$ & Alcohol, cannabis \\
\hline Psychosocial challenges & $\begin{array}{l}\text { Fear of death, absenteeism from school, decreased physical } \\
\text { activities, decreased sexual intercourse, seeking support from } \\
\text { faith and religiosity }\end{array}$ \\
\hline $\begin{array}{l}\text { Sources of emotional } \\
\text { support }\end{array}$ & Siblings, health professionals, religious leaders and teachers. \\
\hline $\begin{array}{l}\text { Sources of psychological } \\
\text { support (agents) }\end{array}$ & Family, school, peers \\
\hline Coping strategies & $\begin{array}{l}\text { Praying, watching television, and surfing the Internet, engaging in } \\
\text { work, gaining financial independence, controlling emotions, } \\
\text { positive self-concept. }\end{array}$ \\
\hline Self-management resources & $\begin{array}{l}\text { Emotional support, family support, advocacy, knowledge of } \\
\text { disease, understanding the disease, understanding self-care, } \\
\text { praying, faith and spirituality, social support, talking to others }\end{array}$ \\
\hline $\begin{array}{l}\text { Interacting with health } \\
\text { professionals }\end{array}$ & $\begin{array}{l}\text { Attending regular medical visits, having knowledgeable health } \\
\text { professionals }\end{array}$ \\
\hline
\end{tabular}

As a result of these limitations the extent to which SCD self-management differs by geographical context, socio-cultural context and personal characteristics such as age and sex 
profile, as well as a generally agreed standard for SCD self-management is unknown. For example, the literature review did not reveal the different needs for adults and children regarding SCD self-management, nor how other SCD related problems such as anaemia and leg ulcers could be self-managed. It was unclear as to what the key components of SCD selfmanagement are. The literature review pointed to the need to utilise mixed method research to study SCD self-management to understand the depth and breadth of SCD self-management.

As part of contributing to addressing this knowledge gap, the current study was conducted in Ghana, a lower middle income country. The study targeted patients with and without health service involvement for their SCD management. Participants also included children, adults, parents and health professionals, who could read and write and some could not. The study explores self-management strategies for other SCD related complications in addition to pain. The next chapter will apply the themes derived from this chapter and other theoretical frameworks to provide a conceptual framework for the study. 


\section{Chapter 4: Conceptual Framework}

The literature review in the previous chapter elicited the common self-management themes for SCD and other genetic diseases. The review showed a lack of an organised body of knowledge regarding generally agreed components of SCD self-management that could serve as a framework for this study. Hence, it was important that self-management for SCD be conceptualised to serve as a lens for the current study. This chapter presents the conceptual framework for investigating self-management strategies for SCD in Ghana. A conceptual framework is a visual or written product that shows concepts to be studied. It identifies the factors, concepts, or variables and the presumed relationship among them. It is a tentative theory of the phenomenon to be investigated [178]. Maxwell [178] maintains that conceptual frameworks are used when no single theories are suitable to inform a given study. They are formed from experiential knowledge, existing theory and research, pilot or exploratory research, and thought experiments. The framework for this study is influenced by previous empirical studies, generic and SCD specific self-management theories, and my personal experience with working with SCD.

In this section, I describe the theories and models, noting key elements, their contribution to the study, and limitations. I then discuss how the concepts in the theories and models are applied to the current study, and present the concepts and definitions. This is followed by a review of literature for each concepts and illustration of how the concept is applied in the study. Finally, I present the conceptual framework for self-management strategies for self-management in Ghana.

\subsection{Existing Theories and Models}

The theories reviewed for this study includes generic and SCD specific as no single framework could adequately address the objectives of the study [19, 32, 175]. The rationale for using a combination of theories was that no one existing theory or model offered a framework that could adequately address self-management of SCD in Ghana.

\subsubsection{Theories of individual and family self-management}

The theoretical concept of self-management takes its roots from grand theories such as cognitive behavioural, health behaviour and social support theories [10, 19]. Currently, the 
construct of self-management has been described in selected frameworks and theories. Two frameworks that specifically theorise self-management and related factors from the individuals and family perspectives are A framework for the study of self-and family management of chronic conditions [32] and The individual and family self-management theory [19]. Both frameworks were developed by nurse researchers in the USA. These frameworks conceptualise self-management as comprising of three dimension - factors influencing self-management (context), process of self-management and outcomes of selfmanagement.

Grey et al.'s [32] framework was derived from an extensive literature review of LTCs. The framework postulates that individuals perform self-management within the context of their family, communities and environment. Self and family management of LTCs is a dynamic process, and is modified by individual outcome, and influence by risks and protective factors. This framework describes the factors influencing SM (individual and context factors), as outcomes for individual and family self-management, but does not describe the processes involved.

Ryan and Sawin's [19] framework was predominantly informed by Grey et al.'s [32] framework and extensive review of self-management literature. This descriptive mid-range theory holds that "self-management is complex dynamic phenomenon consisting of three dimensions. Context, process, outcome" (p. 9). Factors in the context dimension influence individual and family engagement in the process of self-management and directly impacts outcomes. Enhancing the individual and family self-management process results in positive outcomes. This framework further described the process of self-management.

Both frameworks conceptualise factors affecting self-management as comprising individual and environmental (context) factors. Both framework referred to these factors as "risk and protective factors". The individual factors emanate from the disease or an individual's personal characteristics. Disease factors include severity of condition, disease trajectory, complexity of treatment and genetic factors [19, 32]. Personal characteristics include socio-demographic factors such as age, gender, psychosocial characteristics, and diversity factors such as race and culture. According to both frameworks all individual factors can positively or negatively influence self-management. The environmental factors proposed 
by both frameworks include physical environment and social context factors such as social networks, community resources, health care systems, transportation, schools, and culture that may affect the individual's self-management. .

The self-management outcome measures proposed by both frameworks have similarities and differences. The Grey et al. [32] framework categorised outcome variables into individual outcomes (QOL, adherence), health status (control, morbidity, mortality), family outcomes (function, lifestyle) and environmental factors (access, utilisation, provider relationship). In contrast, Ryan and Sawin [19] categorised outcomes as proximal or distal. The proximal are actual self-management behaviours and costs of health care use, and the distal include QOL, health status, direct and indirect cost of health. The distal outcomes result from performance of proximal outcomes. Thus both frameworks agree on health status and QOL outcomes but diverge on the remaining outcomes.

Whiles Grey et al. [32] framework failed to capture the process of self-management, Ryan and Sawin [19] conceptualised the process of self-management as the mechanism underpinning actual behaviours. In describing the process they posits that a person will engage in recommended health behaviour if he has: 1) Knowledge and beliefs consistent with the behaviour to be changed; 2) acquires skills for self-regulation (goal setting, selfmonitoring, reflective thinking, decision making, planning for and engaging in specific behaviours, self-evaluation and management of physical, emotional and cognitive responses associated with health behaviour change); 3) experiences social facilitation (social support, negotiated collaboration between individuals and families and health care professionals). Ultimately, the two frameworks have contributed to the conceptualisation of this study in that they identify they identify the process of self-management, its outcomes and factors that may influence its performance. However, neither frameworks captures the specific behaviours involved in self-management, which is the foundation of this study.

\subsubsection{The theory of self-care management for vulnerable populations}

In SCD self-management literature, the most cited theoretical framework is the theory of self-management for vulnerable populations propounded by two nurses in USA [175]. This SCD focused middle-range theory proposes to address the concept of vulnerability by addressing self-care management and the multiple intra-personal factors that are theorised to 
influence individuals' ability to manage illness. The theory views vulnerable populations as social groups who experience health disparities as a result of lack of resources and increased exposure to risk. This includes people who are made vulnerable by their financial circumstances or place of residence, health, age, personal characteristics, financial or developmental status, communication ability, presence of chronic illness and disability. Such populations include mothers and children, people with HIV/AIDS and homeless families. The theory view people with SCD as a vulnerable population because: the disease is characterised by lifetime manifestations of pain and hospitalisation, patients usually receive inadequate health care and may lack the skills needed to improve self-care and quality of life, patients have inadequate pain relief resulting in poor QOL, patients encounter negative socioeconomic effects (poor academic achievements, interrupted carrier plans, unemployment and poverty) due to the chronicity of SCD.

The theory of self-care management for vulnerable population identify similar concepts for SCD self-management as the generic theories described above. Two types of individual factors are proposed to influence SCD self-management - vulnerability factors and intra-personal resources. Vulnerability factors include modifiable (economic situation, living condition, education) and non-modifiable (age, diagnosis, gender, race/ethnicity) factors. These factors are theorised to negatively affect SCD self-management and health outcomes. Intra-personal factors on the other hand positively influence SCD self-management and includes assertiveness, coping behaviour, knowledge, self-efficacy and social support. The theory also identifies contextual or environmental factors that affect SCD self-management including community values and community resources. Community values are ideas and beliefs that are important to health whilst community resources are assets such as money, political power and cohesiveness within a community. Health outcomes postulated by the theory include health status and quality of life.

The theory did not specify self-management process but defined self-management as "engaging in therapeutic behaviour and implementing social actions to improve health status and quality of life in chronic illness" (p. 47). The theory further holds that increased selfmanagement results in improved health status and quality of life. For adults with SCD the theory identified priority self-management as including: adequate hydration, refraining from exercise exercises, keeping medical appointments, adhering to health professionals' 
instructions, and getting medical questions answered. The central focus in the theory when applied to SCD patients is that people with SCD encounter both modifiable and nonmodifiable vulnerability factors that increase their risk for poor health outcomes and poor QOL. Intra-personal factors mediate the relationship between the vulnerability factors and self-care management, and self-care management is influenced by contextual factors. Improved self-care practices are related to improved QOL and health status in both individuals and populations. Whilst this theory focuses on addressing vulnerability among SCD patients, it views self-care management as a measure that can mediate the relationship between an individual's patient's vulnerability factors, and their health status and QOL. The concepts and relationships in the theory are supported by empirical research $[155,156,172$, 175].

The theory of self-care-management for vulnerable populations and its derivatives greatly informed the framework for the current study. The theory provided insights into factors that can influence SCD self-management and their relationships. The factors influencing self-management identified in the theory (vulnerability factors, contextual factors and intra-personal factors) formed the basis for self-management determinants for the current study. Furthermore, the theory provided understanding about important SCD self-care actions include adequate hydration, refraining from exercise exercises, keeping medical appointments, adhering to health professionals' instructions, and getting medical questions answered. However, the self-management actions outlined appear to favour context with greater health service inputs and may not adequately capture SCD self-management in the Ghanaian setting and similar health systems. The self-management outcomes proposed by the theory are health status and QOL.

In short, the main gap in existing self-management theories (both generic and SCD specific) is that they fail to comprehensively address the specific behaviour required for individual and family self-management. These behaviours are important in understanding the strategies people adopt. 


\subsubsection{Selected self-management models}

As a result of gaps in literature, other self-management models were consulted to understand specific behaviours in self-management. These models included models by Kirk et al. [179] and Schulman-Green [26].

\subsubsection{Process of self-management in long term conditions}

A recent model that described the process of self-management was the processes of self-management in LTCs [26]. A metasynthesis was conducted to address gaps of inadequate knowledge of specific self-management processes and behaviours. The framework identified three categories of self-management process - illness needs, activating resources, and living with LTC. Each of the three process areas consisted of tasks and skills. The tasks represented the essential work involved in self-management whilst the skills represented ways of carrying out the tasks. The model views both tasks and skills as behaviours. According to the model, the process of focusing on illness needs concerns the takes and skills used to care for the body and illnesses. People achieve this process through three key tasks and skills, learning about their chronic illness by seeking information about illness and treatment; obtaining ability to gain control of their health needs through learning to manage their body's responses to illness, and performing health tasks such as keeping to clinical appointments, and performing health promotion activities by engaging in behaviours that reduce the impact of the disease on their lives. With respect to the process of activation of resources, the model indicates this as involving patients' efforts in accessing resources from immediate family, friends, community and the health system to manage their condition. The task and skills involved in this process includes communication with health professional, utilising psychological resources, participation in spiritual groups and soliciting social support.

Regarding the process of living with a LTC, the model posits that individuals develop coping strategies that enable them to adopt to their condition. The individual avoid focusing on their illness by integrating their illness into everyday life. These are undertaken using four strategies including: processing emotions, adjusting, integrating illness into everyday life and making decisions. The model also identified factors that influence self-management including demographics, cultural, clinical and systemic factors. Compared with the theoretical frameworks, this model provided more specific and comprehensive activities involved in self- 
management. Consequently, contributes to the conceptualisation of this study in that it identifies specific self-management tasks and skills that provides better understanding of selfmanagement behaviours and factors that influence self-management.

\subsubsection{Effectiveness of self-care support interventions for children and young people}

The study was also informed by Kirk et al. [179]. In a systematic review to determine the effectiveness of self-care interventions for young people with asthma, cystic fibroses and diabetes, Kirk et al. proposed a conceptual framework to guide the study. According to their framework, self-care management consists of self-care agents, context, self-care activities and factors influencing self-care.

The self-care agents include individual, families, community. The context for self-care could be health promotion, illness prevention, limitation of illness impact, or health restoration. Self-care activities include information seeking, health behaviours to maintain or enhance physical and psychological well-being, self-diagnoses, monitoring and self-treatment. The framework also encompasses managing psychosocial consequences on daily basis, problems solving, seeking advice from lay networks, developing support networks, consulting health professionals, accessing services, developing effective partnership with professionals and active citizenships. The factors that influence self-care could emanate from individual, health services or wider social context. The individual factors include illness beliefs and lay epidemiology, emotional responses to condition, relationship of intentions to change behaviour influences with self-efficacy, self-image, information and skills to change behaviour, processes to implement and normalise behaviour change, levels of disruptions experienced in stages of change, and pre-existing adaptations. The health services influences include nature of professional-patient relationship, design of patient centeredness and health service incentives. The social context influence relates to health system organisation, material and community resources, social incentives and disincentives, and collective support.

It proposed that self-care could be supported by enhancing a positive client-patient relationship, provision of accessible information and education to increase knowledge and skill as well provision of self-care tools and equipment which could be accessed through formal and informal channel. Self-care could also enhanced by interventions directed at health professionals, health systems as well as patients. 
This framework was instrumental in conceptualising the current study as a variety of self-care activities common to people with LTCs were enumerated. Major concepts including health behaviour, self-diagnosis, self-monitoring and self-treatment were identified in this framework. It also outlines the factors that can influence self-care. However, the framework did not explain the concepts and the relationships between them. Hence could not be adopted solely for this study.

\subsubsection{Self-management of long term conditions by older adults}

Another model that informed the study was Clark et al. [23]. These authors reviewed self-management tasks for heart disease, asthma, arthritis, COPD and diabetes to identify common tasks (outlined in chapter one). These tasks enhanced understanding of common strategies patients might use, and were important in informing the conceptualisation of the actions or behaviours. However, no relationships and descriptions were provided regarding the tasks, hence it was inappropriate to adopt this model as the framework for the current study.

\subsection{Application of Modules and Concept to Current Study}

The theories and models helped to identify the components of self-management and their relationships. Majority of the self-management actions, process, tasks and skills can be organised as Preventive health, self-monitoring, self-diagnosis, self-treatment, self-evaluation, and factors influencing self-management as contextual and vulnerability factors. These concepts therefore formed the components of the conceptual framework for the current study (Table 6).

\subsubsection{Preventive health}

In this thesis preventive health behaviours concerns actions patients and families take on a daily basis to promote their health and prevent exacerbation of disease. In line with the literature these measures included health promotion measures such as nutrition and hydration and preventive measures such as avoidance of exercise exercises. In the SCD selfmanagement literature, preventive measures are often described as part of general self-care $[172,175]$. Utilisation of these measures has been linked to improved health status, QOL and a reduction in health costs [172]. In the theory of self-care management, preventive measures 
investigated for SCD were adequate hydration, refraining from exercise exercises, keeping medical appointments, adhering to health providers' instructions, getting medicinal questions answered. From my experience these measures may not adequately capture preventive health strategies in Ghana given the context. Consequently, these measures were comprehensively explored from participants' perspectives using qualitative and quantitative approaches.

TABLE 6. CONCEPTS AND DEFINITIONS

\begin{tabular}{|c|c|}
\hline Concept & Definition \\
\hline $\begin{array}{l}\text { Preventive Health } \\
\text { behaviour }\end{array}$ & $\begin{array}{l}\text { Actions patients and families take on a daily basis to promote their } \\
\text { health and prevent exercitation of disease. }\end{array}$ \\
\hline Self-monitoring & $\begin{array}{l}\text { Techniques patients and families use to continuously observe for } \\
\text { indicators for possible problems and potential triggers of crisis and } \\
\text { other complications. }\end{array}$ \\
\hline Self-diagnosis & $\begin{array}{l}\text { Medical signs and symptoms individuals use to recognise SCD } \\
\text { related illnesses or problems they consider problematic which } \\
\text { warrants intervention and means of recognising these. }\end{array}$ \\
\hline Self-treatment & $\begin{array}{l}\text { Therapeutic actions (pharmacological and non-pharmacological) } \\
\text { patients and families take to manage or treat a recognised problems } \\
\text { on their own. }\end{array}$ \\
\hline Self-evaluation & $\begin{array}{l}\text { Patients' perceptions of the impact of their self-management } \\
\text { actions. }\end{array}$ \\
\hline Contextual factors & $\begin{array}{l}\text { Social and environmental factors that affects SCD care. These } \\
\text { include community beliefs about SCD and related illnesses, access } \\
\text { to SCD services, cost of health services }\end{array}$ \\
\hline $\begin{array}{l}\text { Vulnerability } \\
\text { factors }\end{array}$ & $\begin{array}{l}\text { Individual personal characteristics that can affect the care of SCD. } \\
\text { These include } \\
\text { - Age } \\
\text { - Annual income (economic situation) } \\
\text { - Employment status } \\
\text { - Gender } \\
\text { - Living conditions (rural or urban) } \\
\text { - Complications of SCD } \\
\text { - Number of crisis }\end{array}$ \\
\hline $\begin{array}{l}\text { Self-management } \\
\text { resources }\end{array}$ & $\begin{array}{l}\text { Resources patients possess to enhance their SCD care. These } \\
\text { include knowledge of SCD, knowledge and skills for SCD self-care, } \\
\text { social support, medicinal and non-medicinal logistics for SCD care }\end{array}$ \\
\hline Health status & people's perception of how their health is \\
\hline Quality of life & people's perception of their overall well being \\
\hline $\begin{array}{l}\text { Self-management } \\
\text { strategies }\end{array}$ & $\begin{array}{l}\text { The actions patient and families take by themselves on a daily basis, } \\
\text { to maintain their health, prevent complications, check the progress } \\
\text { of their health, recognise problems, and manage problems. }\end{array}$ \\
\hline
\end{tabular}




\subsubsection{Self-monitoring}

Self-monitoring is the most developed concept in self-management literature. Selfmonitoring has been conceptualised as encompassing: 1) self-measurement of vital signs, symptoms, behaviours or psychological well-being; 2) self-interpretation of these data; and 3) self-adjustment of medication, treatment, lifestyle, or health seeking behaviour as a result of self-monitoring or self-interpretation [180]. Self-monitoring was captured as selfmeasurement and self-adjustment of diet and medications in diabetics [12]

Self-monitoring involving self-measurement and is common among LTCs such as diabetes and hypertension. In studies on hypertension, self-monitoring involved selfmeasurement of blood pressure and self-titration of medication [181]. In diabetes, selfmonitoring involved self-measurement of blood glucose [182]. One study found that selfmonitoring of blood glucose lead to improvements in glycaemic control and reduction in cardiovascular risks due to diabetes [183]. Among patients with heart failure, self-monitoring involved the use of a diary to record daily weight and symptoms. The findings showed that diaries users had improved clinical and hospital outcomes [184].

In the SCD literature, self-monitoring included self-measurement of pain, observation of physical sensations, and monitoring of pain triggers. Use of diaries was common among the studies reviewed. In the current study, self-monitoring is defined as techniques patients and families use to continuously observe the progress of their disease, possible problems and potential triggers of crisis and other complications. It includes monitoring or observing physical sensations (symptoms), physical signs, self-measurements and self-observations of triggers.

\subsubsection{Self-diagnosis}

The concept of self-diagnosis as well as self-monitoring and self-evaluation are described in cognitive behavioural theory as strategies for human self-regulation [185]. These mechanisms have been specified as important for disease self-management [10, 19, 23]. Selfdiagnosis was conceptualised in the cognitive behaviour theory as self-insight which results from self-monitoring. This term has had limited application in chronic disease selfmanagement literature compared to self-monitoring. Self-diagnoses was specified as self-care activity in Kirk et al. [179]. Clark et al. [23] also identified symptom recognition as part of 
self-management. In some studies, elements of self-diagnosis such as symptom recognition were captured as part of self-monitoring [180]. In the literature review for the current study, self-diagnosis was denoted as self-interpretation and classification of pain. In this thesis, selfdiagnosis is captured as medical indicators used by patients and careers to recognise impending or actual SCD related problems or complications. The focus is on medical signs and symptoms patients consider as problematic and warrants interventions.

\subsubsection{Self-treatment}

Self-treat was identified in Kirk et al. [179] as part of self-care activity but no explanation was provided. Similar to self-diagnosis, the term has had limited use in chronic disease self-management literature. As demonstrated in the literature review, patients may use pharmacological, and non-pharmacological strategies to manage recognised problems. In this study, self-diagnosis included administration of all therapeutic strategies to manage recognised, SCD illness, and complications.

\subsubsection{Self-evaluation}

Self-evaluation concerns assessment of effectiveness of patient's self-treatment from their own perspectives. It has been theorised by the Social Cognitive Theory of SelfRegulation [185] that people pursue courses of action that produce positive effect and refrain from behaving in ways that result in self-censures. On the basis of this, it was anticipated that SCD patients will engage in actions that give them positive effect and refrain from actions that are unhelpful. Consequently, this study investigated the impact of self-management actions from patients' perspectives in order to identify helpful and unhelpful actions. Knowledge of helpful self-management actions will be important in developing helpful interventions.

\subsubsection{Factors influencing self-management}

The study investigated factors that have been theorised to influence a person's selfmanagement. In order to align with existing theory, these factors have been conceptualised as vulnerability factors, contextual factors and self-management resources. Vulnerability factors investigated were age, income, employment, gender, living condition and complications of SCD. Contextual factors included community beliefs and resources. Community beliefs 
include understandings and ideas about SCD and related illnesses, community resources include geographical and financial access to health services for SCD care, and other social services people seek to support their self-care. Self-management resources investigated in this study included social support available to SCD patients, knowledge of SCD and self-care, and materials resources such as medicines and non-medicines logistics available at home for their SCD care.

\subsubsection{Outcome}

Self-management outcomes investigated in this study were health status and QOL.

These were shown in the literature review as established self-management outcomes. Figure 2 illustrates the conceptual framework for SCD self-management in Ghana that was derived from the literature review and the examination of theories regarding self-management. The framework indicate that persons with SCD perform self-management actions to manage their physical and psychological health (tasks, activities, skills, behaviours) and these actions can result in improvements in health outcomes. The self-management actions are influenced by some environmental or contextual factors and intra-personal resources.

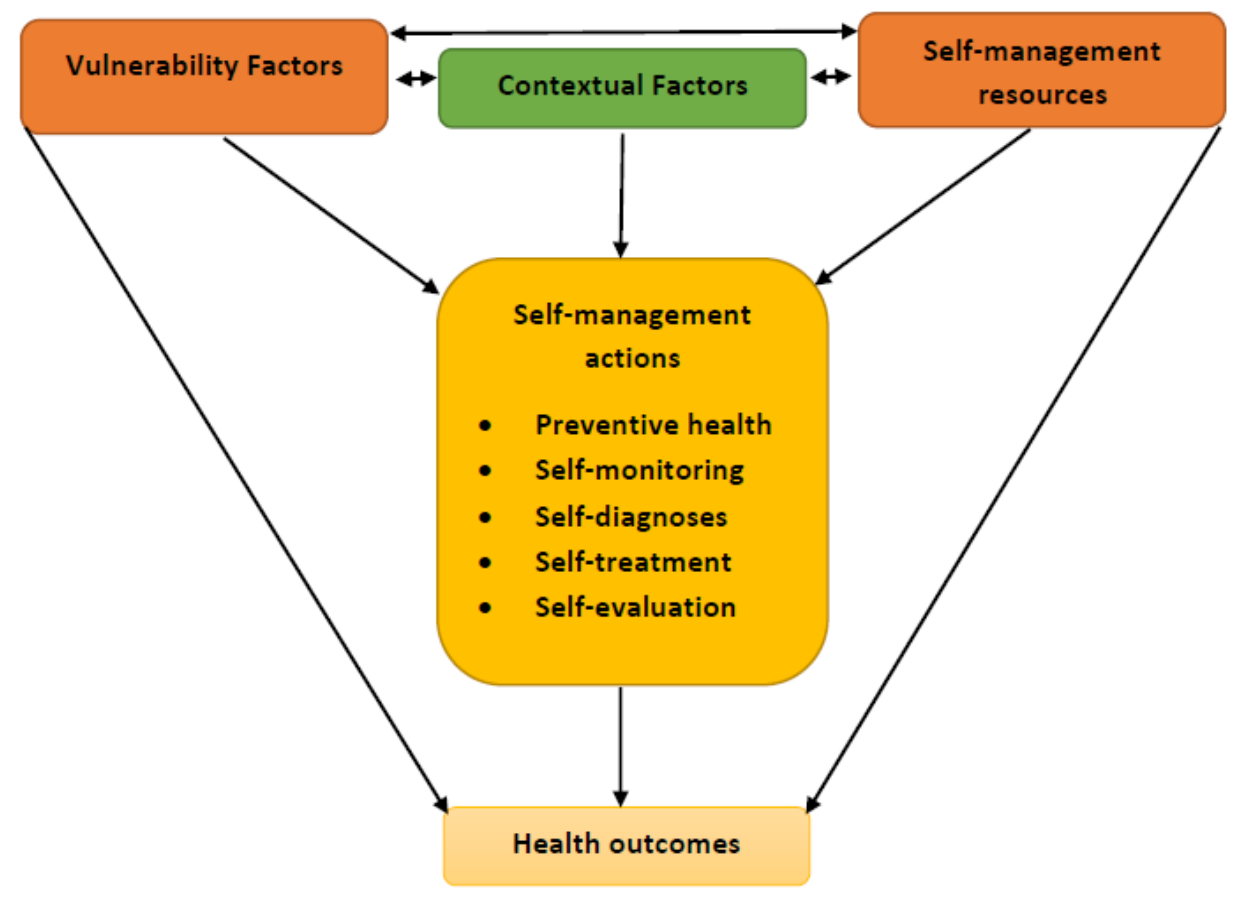

FigURE 2. CONCEPTUAL FRAMEWORK FOR SELF-MANAGEMENT STRATEGIES FOR SICKLE CELL DISEASE IN GHANA 
The conceptual framework proposes that SCD patients or carers performs five key interactive self-management actions including: preventive health behaviour, self-monitoring, self-diagnoses, self-treatment and self-evaluation. Self-management actions impact on the health status and QOL of SCD patients. However, contextual factor s (cost of health services, availability of sickle cell centre to client, closeness of any hospital or clinic to the clients), vulnerability factors (ability to self-monitor, self-diagnose, self-treatment and adopt preventive behaviours) and self-management resources (knowledge of SCD, social support) available to individual patients or families influences the performance of self-management actions.

In applying this framework to this current study, self-management actions explored included what patients do on day-to-day basis to prevent the occurrence of illnesses, monitor their disease or triggers, diagnose or recognise signs and symptoms that warrant interventions, and manage or respond to recognised illnesses. The study also investigated patient's perceptions of the impacts of their self-management actions, self-management resources they possess, contextual and vulnerability factors and how these affect self-management.

The conceptual framework for SCD self-management for Ghana had a role in informing the data collected for understanding self-management of SCD in Ghana. The methodology and methods used in the research are described in the following chapter. 


\section{Chapter 5: Methodology}

This chapter describes the strategies and methods used to answer the research question. The chapter includes an overview of mixed method research, philosophical foundation and rationale for the choice of an exploratory sequential design for the study. It also describes the steps used to recruit the sample, develop instruments, collect and analyse the data, and the procedures used to achieve ethical standards, rigour and trustworthiness of the study. The chapter is organised into two phases -qualitative and quantitative. The description of the phases and sequencing of data collection and analysis are summarised in diagrams. Each phase commences with its purpose, objectives and methods and ends with a conclusion, summarising the key actions for the next stage.

\subsection{Overview of Mixed Method Research}

Mixed method research, generally viewed as third research paradigm brings together quantitative and qualitative methods to tell a holistic research story. Despite being a relatively new approach to research, mixed method research has gained popularity in a variety of disciplines including health and nursing [186]. By combining both quantitative and qualitative methods in a single study, mixed method researchers have the advantage of minimising weaknesses associated with using quantitative or qualitative methods alone, by triangulating results from more than one method, thereby providing higher quality evidence. Moreover, mixed methods offers a practical way of tackling research problems that require reporting of experiences and trends. a researcher can use multiple paradigms and can employ inductive and deductive approaches to a single study $[186,187]$.

\subsubsection{The philosophical worldview of the study}

The choice of research approach is influenced by the researcher's philosophical worldview or paradigm which is the researchers perception about the world [187]. Worldviews include pragmatism, critical realism, transformative-emancipatory, positivist, constructivist or use of multiple worldview [186, 187]. Mixed method researchers can use single or multiple worldviews. It is argued by Creswell and Plano that single worldviews are suitable for concurrent designs and multiple worldviews for the sequential designs. 
Researchers using the sequential designs are typically required to use constructivist worldviews for the qualitative phase and post-positivist for the second phase.

Although the current study was sequential, and warranted the use of multiple worldview as recommended by Creswell and Plano [186], pragmatism, which is a single paradigm was used to guide the study. The basic ideas underpinning pragmatism are action, situation, and consequences rather than antecedent. To the pragmatist, truth is not absolute unity but rather what works at a given time [187]. As a result, emphasis is placed on what solution would work best for a given problem. Pragmatism was chosen as it offered a realistic approach to answering the research question. It meant I could focus on the problem and use several methods to derive knowledge about self-management of SCD rather than focusing on debates about metaphysical concepts such as truth, reality as required by other worldviews.

\subsection{Design for the Current Study}

Creswell and Plano [186] discuss two variants - theory development and instrument development - for exploratory sequential mixed method design. In the theory development variant the qualitative phase is conducted to develop emerging theory, taxonomy or classifications system, and the findings are examined in a larger quantitative study. The researcher places emphasis on the initial qualitative stage and the quantitative plays a secondary role. In contrast with the instrument development variant, the qualitative phase informs the development of instruments for the second and larger quantitative phase. This design enables researchers to explore the dimensions of their topic with different population groups, usually starting with a small sample, and using the findings from this sample to develop additional research instruments which can be used on larger populations. Creswell and Plano [186] maintain this variant is useful when measures or instruments are not available for the concepts the researcher wishes to study, the variables are unknown, or no guidelines or frameworks exist. The instrument development variant is also useful when the researcher wishes to generalise the findings to a larger group, explore a phenomenon in-depth and measure the prevalence of its dimensions, or test aspects of emerging theory or classifications.

The advantage of the sequential mixed method emanates from separate phases making the design simple to describe, implement and report. However, researchers using sequential mixed methods can be confronted with challenges as such studies require considerable time to 
conduct, there can be difficulties (such as gaining ethical clearance) associated with specifying the procedure for the quantitative phase and in deciding which aspects of the qualitative phase data to use for the quantitative phase. Other challenges concern developing procedures to ensure validity and reliability of the results [186].

The current study is based predominantly on the instrument-development variant. The choice of this design was influenced by the gaps in literature, the nature of the research question, and indications of best practice in the self-management literature. As a gap exists in literature regarding SCD self-management and there were no evidenced based-instruments that suitable to investigate SCD self-management in Ghana, an instrument-development design was adopted to identify variables and concepts suitable for the Ghanaian context. Additionally, this design was suitable for a comprehensive exploration of the research question. The initial qualitative phase lead to the discovery of issues central to selfmanagement and broader insights into the mechanisms involved. The quantitative phase enabled the collection and quantification of self-management practices and issues identified in the qualitative phase. It also enabled the performance of statistical analysis to establish the relationship between self-management and respondents' socio-demographic characteristics. Ultimately, the combination of individual experiences in the qualitative phase and quantification of perspectives in the quantitative phase lead to better insight into selfmanagement strategies in Ghana.

Best practices in self-management literature indicate that self-management practices are problem-specified and contextually different for different populations. Hence, Lorig et al.'s [36] observes that self-management practices identified in one setting cannot be transferred directly to another. Consequently, self-management experts recommend a needs assessment is conducted before self-management interventions are implemented in any given setting. According to Lorig et al. baseline assessment is essential to identify patients' attitudes towards the disease and life as well as the skills they can use in managing challenges of the condition. For example, before the development of the arthritis self-management programme in USA, baseline assessments were conducted with the aim of uncovering the most useful themes and tasks to include in the programme $[23,36]$. Furthermore, in places where the arthritis programme was replicated, further needs assessments were usually conducted. These needs assessment led to modification of the programme content in countries such as Japan, 
China and Vietnam. Although, some knowledge of SCD self-management exists in USA, Brazil and Jamaica, this knowledge cannot be directly transferred to the Ghanaian context, hence the need to conduct this study.

\subsection{Overview of Phases, Stages and Methods for the Study}

The exploratory sequential mixed method design involved two phases (quantitative and qualitative) and four stages of data collection sequenced as follows: 1) review of SCD focused websites, 2) interviews with health professionals with expertise in SCD management in Ghana, 3) interviews with patients and parents of children with SCD, and 4) survey of adult SCD patients and parents of children of SCD children (Figure 3).

\section{Qualitative Phase}

Quantitative Phase

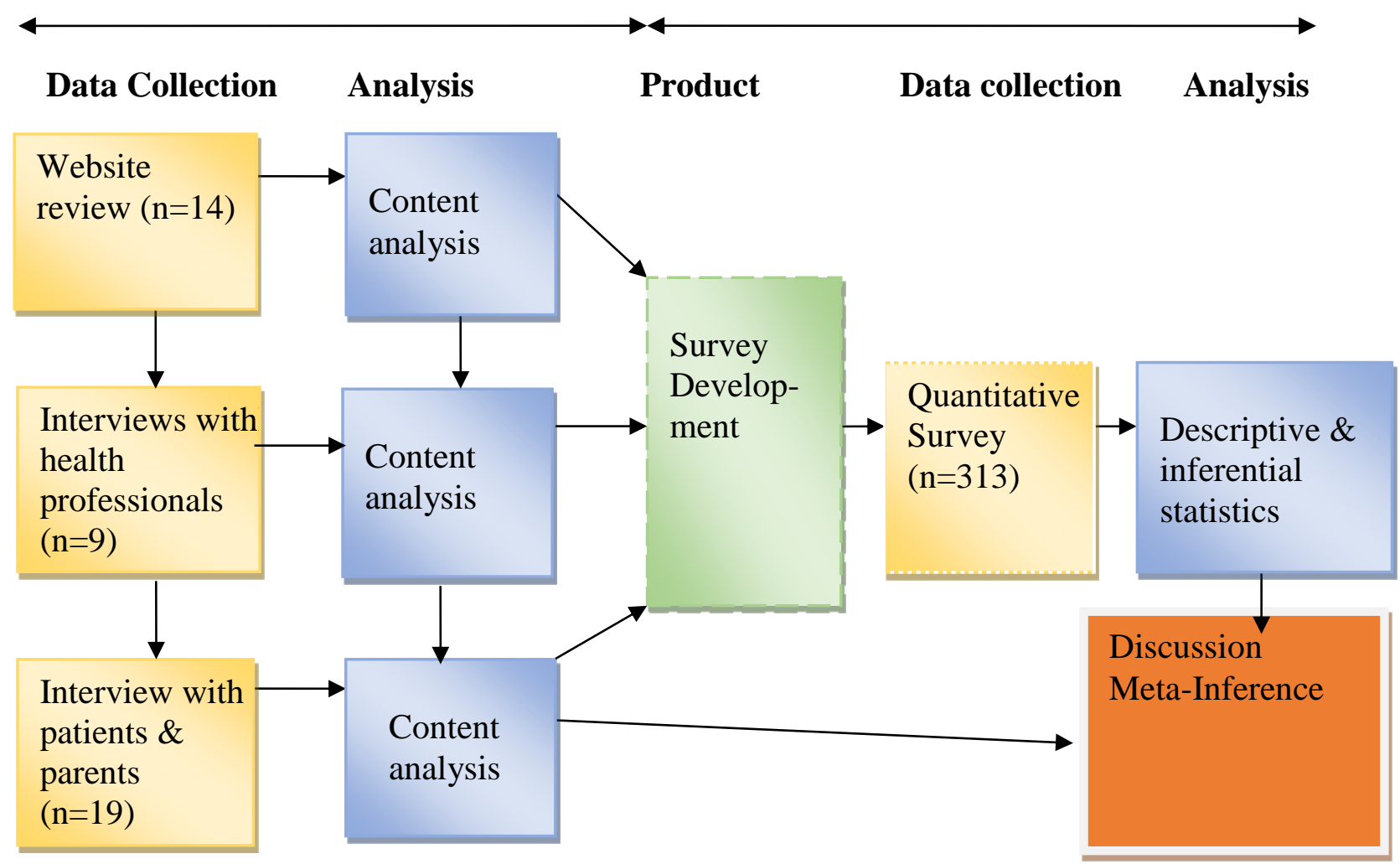

Figure 3. PhaSES AND STAGES OF DATA COLLECTION, ANALYSIS AND INTERPRETATION 


\subsection{The Qualitative Phase}

The qualitative phase involved websites review and the interviews with health professionals and with adult patients and parents of children with SCD. The methods and procedures used for each stage are discussed in the subsequent sections. Qualitative content analysis (CA) was used as the method for data collection and analysis for all the qualitative stages but applied differently for the website review and the interviews. This section presents an overview of qualitative CA and it application to the qualitative stages.

\subsubsection{Overview and rationale for using qualitative content analysis}

Content analysis has become a fast growing method in qualitative research especially with the emergence of computer technology [188]. The method originated in journalism in the 1950s but is currently applied widely in many disciplines including health and nursing due to its flexibility [188, 189]. It is essentially a method for analysing text data and can be applied quantitatively or qualitatively depending on the theoretical perspective and nature of research question [190]. Neuendorf [188] defines CA as "the systematic, objective, quantitative analysis of message characteristics” (p. 1). Alternatively, Berg [191, p. 268] views CA as the process of "systematically and objectively organising qualitative data" in such a way that it can be quantitatively counted. Kim et al. [192] argue that Qualitative CA is applicable to many areas of inquiry including naturally occurring language, newspaper coverage, advertisements, symbols and artefacts. In nursing, qualitative CA is frequently utilised [193, 194]. It has been used to examine teaching [195, 196], and trends in theses, dissertations and publications [197-200]. It has also been applied to evaluate student learning and practice [201], and to examine specific nursing information in textbooks and other teaching and learning materials [202, 203]. It has also been used to evaluate nursing interventions [204, 205 ] and to assess the image of nursing in the media [206].

CA has several advantages. It is unobtrusive and enables the examination of artefacts and large volumes of data relatively easily. Its capability to analyse large volumes of data makes it especially suitable for analysing web-based content [207]. It is cost effective since existing data can be used as compared to other methods like interviews. Its major challenges include difficulties in locating information relevant to research questions and its ineffectiveness in establishing cause effect relationships [191]. This study was not confronted 
with such challenges because the research question was already established and CA was used as an analytic strategy rather than a complete research strategy. Furthermore the CA was applied in the study descriptively to only describe relationships.

Quantitative CA is distinguishable from qualitative CA in that quantitative CA tends to begin with predetermined codes and uses search algorithms to apply the codes to the text [208]. The key intent of quantitative CA is to count text without regard to context, and analysis stops with numeral interpretation. In contrast, qualitative CA uses an inductive approach in both coding and interpretation of counts. The counts serve as the first step in interpreting the patterns, followed by a further examination of the patterns to produce a theory or description that explain the patterns [209]. The emphasis is on understanding the new context that is revealed by the coding and counting process.

Hsieh and Shannon [210] identify three types of qualitative CA - conventional, directed and summative. Directed and summative CA were utilised in this research. Directed CA is a deductive approach aimed at extending or validating a conceptual or theoretical framework. It is guided by a structural process and suitable when a study begins with an existing theory or conceptual framework. The advantages of directed CA is that existing theories help to focus the research, provide direction about the variables and facilitate identification of initial codes and relationships between the concepts and variables. The challenge is potential bias as a researcher approaches the data through the lens of a theory or framework, which may cause the researcher to find mainly supportive evidence of the theory. It also allows the researcher to ask questions without regard to the context of the phenomenon. Some participants may also discover cues and answer questions in a particular way to please the researcher. In recognition of these factors, I was careful in following recommended steps to reduce possible bias.

According to Hsieh and Shannon [210] summative CA involves quantifying the frequency of certain words and interpreting the content from which the word was located. The purpose is to understand the contextual use of words in texts. The qualitative phase of the current study used features of directed and summative CA for the website review but only the directed CA was used for the interviews. Table 7 summarises the publications that informed the application of CA for the current study. 
TABLE 7. PUBLICATIONS USED TO INFORM THE METHODS OF THE CURRENT STUDY

\begin{tabular}{|c|c|c|c|c|}
\hline $\begin{array}{l}\text { Deductive Content Analysis } \\
\text { Elon \& Kyngäs (2008) }\end{array}$ & $\begin{array}{l}\text { Directed Content Analysis } \\
\text { Hsieh \& Shannon (2005) }\end{array}$ & $\begin{array}{l}\text { Summative Content Analysis } \\
\text { Hsieh \& Shannon (2005) }\end{array}$ & $\begin{array}{l}\text { Web Content Analysis } \\
\text { McMillan (2000) }\end{array}$ & $\begin{array}{l}\text { Web-Based Content } \\
\text { Analysis } \\
\text { Kim \& Kuljis (2010) }\end{array}$ \\
\hline $\begin{array}{l}\text { 1. Transcribing interviews } \\
\text { 2. Selecting unit of analysis } \\
\text { (each interview) } \\
\text { 3. Familiarity with data/ } \\
\text { making sense of the data } \\
\text { 4. Developing } \\
\text { categorization matrix } \\
\text { 5. Review data for content } \\
\text { 6. Coding data into } \\
\text { categories } \\
\text { 7. Selecting text unit to } \\
\text { corresponding categories } \\
\text { 8. Developing } \\
\text { subcategories/ new } \\
\text { categories } \\
\text { 9. Applying subcategories } \\
\text { developed back to text } \\
\text { (focused coding) } \\
\text { 10. Reporting findings }\end{array}$ & $\begin{array}{l}\text { 1. Establishing existing } \\
\text { theory or prior research } \\
\text { 2. Identifying initial key } \\
\text { concepts/variables as } \\
\text { coding categories } \\
\text { 3. Operationalise category } \\
\text { definitions using theory } \\
\text { 4. Reading transcripts } \\
\text { 5. Coding categories } \\
\text { 6. Developing new } \\
\text { categories from text } \\
\text { which did not fit into } \\
\text { existing categories } \\
\text { 7. Writing findings based on } \\
\text { frequency of } \\
\text { supporting/non- } \\
\text { supporting evidence of } \\
\text { theory/framework } \\
\text { 8. Discussing findings } \\
\text { within the context of } \\
\text { theory or framework }\end{array}$ & $\begin{array}{l}\text { 1. Searching for identified } \\
\text { words (computer or } \\
\text { manually } \\
\text { 2. Calculating word } \\
\text { frequency by source or } \\
\text { speaker } \\
\text { 3. Interpretation of context } \\
\text { associated with of words } \\
\text { or phrases }\end{array}$ & $\begin{array}{l}\text { 1. Formulating research } \\
\text { questions or hypothesis } \\
\text { 2. Sample selection } \\
\text { 3. Defining categories } \\
\text { 4. Training coders and } \\
\text { checking reliability of } \\
\text { their scores } \\
\text { 5. Analysis and } \\
\text { interpretation of data }\end{array}$ & $\begin{array}{l}\text { 1. Formulating research } \\
\text { question } \\
\text { 2. Identifying variables } \\
\text { 3. Defining categories and } \\
\text { units of measurements } \\
\text { 4. Creating coding scheme } \\
\text { 5. Sampling and data } \\
\text { collection } \\
\text { 6. Coding into categories } \\
\text { 7. Selecting text into } \\
\text { categories } \\
\text { 8. Developing subcategories } \\
\text { 9. Applying subcategories } \\
\text { back to text } \\
\text { 10. Reporting }\end{array}$ \\
\hline
\end{tabular}




\subsubsection{Stage 1: Review of SCD focused websites}

The first stage of data collection and analysis involved a CA of international SCD focused websites. Qualitative CA was applied as little was known about the scope of SCD self-management behaviours. Therefore, this stage of the study was designed to identify variables recommended for self-management behaviours, and inform the second stage of the study.

The internet or World Wide Web has become an important source of unobtrusive data for CA since 1990s. CA is most suitable for web data since such data are unstructured with different formats [192]. Features such as hyper textuality, interactivity, chaotic structure, large number of pages, multiple media, rapid growth and change of the web present special challenges to web CA [188]. In addition, the lack of appropriate guidelines on website sample selection, and definition of unit of analysis makes sampling, coding and generalisability difficult [188, 192, 207]. Kim and Kuljis [192] argue these challenges can be overcome through creativity.

As limited guidelines exist for conducting web CA, the procedure for this study was informed by recommendations from publication on qualitative CA $[210,211]$ and web CA $[192,207]$. In addition, the study was informed by general recommendations for quantitative and qualitative data analysis [212]. As this study is guided by a conceptual framework, the directed and summative CA approaches were combined for the website CA.

Eleven steps were followed to conduct the study: 1) Formulating research question, 2) Identifying variables, 3) Defining categories, 4) Defining units of measurement, 5) Creating coding scheme, 6) Identifying study population, 7) Sampling, 8) Data collection and extraction, 9) Coding and selecting text into corresponding categories, 9) Developing subcategories and applying subcategories back to text, and 11) Analysis and reporting.

1) The research question at stage one was "What do the websites recommend for dayday living for persons with SCD in terms of keeping well, reducing complications and managing complications when they occur". It was anticipated that identifying recommendations for daily living would provide a new perspectives into the scope of self-management behaviours for patients and families. 
2) The variables to conceptualise the study were derived from review of previous research, theories and models presented in chapters (lit review and conceptual chapters).

3) The categories were formed from the components of the conceptual framework (Chapter 4). These included self-management actions (preventive health and maintenance behaviours, self-monitoring, self-diagnosis, self-treatment, and selfevaluation) and self-management determinants (vulnerability factors, contextual factors, and self-management resources). These categories served as lenses for data collection and analysis.

4) Researchers conducting web CA are required to establish the units of data collection and analysis or variables to be measured, analysed and reported [188, 207]. Choosing units of measurements presents special challenges for web CA because of multiple media in the site. The researcher must decide whether to analyse all pages of the site, home page or opening screen [192]. In this study, the individual websites (all pages) constitute both the unit of data collection and analysis. However, only messages meeting the inclusion criteria were included.

5) The coding scheme comprised a four column codebook and a two column coding sheet that captured categories, variables, measurements, and codes. It was initially developed from concepts and variables from the literature review and finalised after reading through the data retrieved from the websites. The coding sheet contained categories and subcategories and their corresponding data, copied from the websites

6) The study population is the set of units being studied to which the researcher wishes to generalise [186]. In CA, the population is usually the set of messages or a population of people whose messages are being collated and analysed [188]. The population for this study were the SCD focused websites including: associations, clubs, societies, organisations, and foundations. The websites eligible for selection were those that provided advice regarding clinical and psychosocial support, awareness, information, education and communication materials (IECMs) to patients, families, and communities affected by SCD. The exclusion criteria were websites that focused only on charity, fundraising or information directed at health professionals only. 
7) Sampling. The WWW was searched using Google and Yahoo search engines. The use of these search engines was appropriate as a list for SCD associations could not be located either online or offline to serve as a sample frame. Furthermore, McMillan [207] argues using search engines helps to obtain updated websites as compared to offline sources that may contain outdated information. Thirdly, these search engines lead to an efficient compilation of websites that meet specific inclusion criteria as compared to human list compilers [207]. The search terms used for both search engines were: "sickle cell" AND (associations OR societies OR clubs OR organisations OR foundations). Google search was conducted on the 9th of May, 2014 and yielded 2,630,000 results. The search in Yahoo was conducted on the 11th of May, 2014 and resulted in 86,700 results. The outputs of the search were manually screened in the order they appeared and selection was based on the titles of the websites. Sites with titles consistent with the inclusion criteria were selected. Each selected site was then scanned to identify relevant materials. The selection was stopped approximately after 100 outputs as no new relevant sites were being added. The selection of the sites was conducted between the 9th and 30th May, 2014. Fourteen websites were identified and included in the study.

8) Data collection and extraction. At each website, data was collected from information, education and communication materials including web pages, audiovisuals, and printable materials such as books, posters and leaflets. Thirty three relevant materials were located from the 14 websites. Once the materials were located, the content was extracted from messages that provided information to patients and guardians on keeping well, reduction of complications, and managing problems. These extractions included sections, pages, sentences, paragraphs and words. Data extracted included coding units (smallest segment of content counted and scored) and context units (body of materials surrounding the coding units) [207]. Text data were extracted into Microsoft word format, videos were viewed and relevant points written in word format.

9) The messages were coded based on the categories and subcategories using comment boxes or pen and paper. Quotations reflecting each variable were copied from the context units and placed against the corresponding categories and subcategories in the 
data extraction sheet. Message under each subcategory were then numbered as subunits.

10) The sub-categories were reviewed after reading through the data several times. The subcategories were applied back to the texts to test for fitness. This resulted in collapsing, removing or adding some subcategories but no new main category was added.

11) Given that the study was a summative $C A$ [210], both quantitative and qualitative data analysis were conducted. The data under each subcategory were numbered consecutively and counted to generate quantitative results (counts and percentages). The corresponding quotations for each variable (context units) were summarised and describe in the narrative to support the quantitative results. The results were described through themes that emerged from the subcategories as recommended by Hsieh and Shannon [210]. Results were described using the rank order comparison of frequency of quotes.

The themes derived from this stage were used as overarching topics for the interviews with the health professions and with the patients and parents of children with SCD. The new variables and concepts were used to guide the interview questions.

\subsubsection{Stage 2: Health professionals' interview}

Stage 2 involved interviews with health professionals with experience in SCD management. The purpose of these interviews was to describe health professionals' recommendations for SCD self-management in Ghana, and elicit further insight into SCD self-management within the Ghanaian context. According to Clark et al. [23] involving health professionals in patient self-management research is important because health professionals can provide information on the self-management strategies they recommend, what is useful and their insights into what people use. I interviewed nine health professionals asking 1) What they ask patients to do to keep well, 2) how they ask patients to monitor their health, 3) how they advise patients to recognise a sickle cell related problem, 4) what actions they advise patients to take when there are problems, and 5) what patients self-management practices they have observed from their experiences. 


\subsubsection{Methods}

Semi-structured interviews was conducted as little is known about self-management in Ghana and people's experiences had to be explored in-depth. Interviews are suitable when there is need to probe into responses to obtain detailed descriptions and explanations of experiences, behaviours and beliefs of a people; and obtain information that are not anticipated by the researcher [213]. Qualitative CA was used to analyse the data.

Nurses and doctors who work with SCD patients at the SCD clinic or Sickle Cell Association (SCA) were purposively recruited [214]. To be eligible for selection they had to have at least three months experience in providing clinical care, patient focused education, or psychosocial care to patients and families. As data had to be collected via phone interviews from New Zealand, a local coordinator assisted with recruitment and other local arrangement. The coordinator first contacted the potential participants to inform them about the study. I followed up with phone calls and e-mails to solicit their agreement to participate in the study. In all, 10 professionals (nurses 6, physicians 3, physician assistant 1) were contacted and all declared their willingness to participate. However, one doctor could not be interviewed due to their travel commitment.

A six semi-structured interview guide was used to guide the interview (Appendix 12). The guide was tested on a nurse and a doctor initially and minor changes were made. These two interviews were included in the analysis. At the pre-arranged time the interview began with a repeat explanation of the research. The interview bean with an overarching question "what do you tell your clients with SCD and their families to do for SCD care at home?" The aim of this question was to solicit as much narration as possible regarding self-management. This question was followed by targeted questions to elicit responses for the various categories. Specific probes were used to further explore answers for relevant categories. Probing techniques such as "could you explain further" were used where necessary.

The consent forms and information sheet were sent to participants through the local coordinator who also received the completed consent forms. Three participants signed informed consent forms and the others gave verbal consent. Participants who opted for verbal consent were asked to go through the formalities for the purposes of recording before the interviews. However, some declined the formalities since they had already read the 
information sheet and were satisfied with the information. Participants were informed of tape recording immediately before the main questions. As noted by Trochim and Donnelly [214] this is the best time to declare this information as participants have already been vested in the interview.

Following interviews data were transcribed by the primary researcher. The procedures for data analysis were informed by directed or deductive qualitative CA [190, 211]. The analysis began with verbatim transcription of interviews. In order to ensure familiarity and make sense of the data, the transcripts were read several times to obtain an overall picture of the recommendations by each professional. Each interview served as the unit of analysis for the study. According to Graneheim and Lundman [215] a whole interview is preferred since it is normally large enough to capture a person's account and small enough to be kept in mind as context for meaning unit.

Regarding categorisation matrix, the concepts in the conceptual framework were used as described in the previous section. A deductive approach was used as Hsieh and Shannon [210] argue that if the intent of the study is not to capture all aspects of the phenomenon including emotions, then the researcher begins coding immediately using the predetermined categories. In line with this, using the conceptual framework as a lens, the data was initially coded using Nvivo 10.22. Aspects of the data that addressed categories were sent to the respective categories. Data that did not fit into any category were sent to "other" category. Thereafter, the categories were further analysed to derive subcategories using the codebook as a guide. The other category was visually scanned to identify emerging issues. Further subcategories were identified through this process, but no new main categories were identified. The sub-categories were then applied back to the data to test for fitness.

The findings were reported using themes derived from the sub-categories. The results were also organised based on frequency of responses in rank order fashion. All new variables identified were added to the pool of items derived from the literature review and websites review for use in the survey. The findings also informed the interview schedule for the patients and parents. 


\subsubsection{Stage 3: Patients and parents interviews}

Stage 3 was interviews with people with SCD and parents of children with SCD. The objective of this stage was to explore the experiences of patients and parents regarding their day-to-day management of SCD and to identify further variables and concepts for the subsequent stage.

The study involved semi-structured interviews conducted via phone or face-to-face (Appendix 13). Qualitative CA as describe in the health professional's interview was utilised to analyse the data. The inclusion criteria were: 1$)$ Adult ( $\geq 16$ years) patient with diagnosis of any type of SCD who attend the sickle cell clinic or a SCA meetings. It was expected that adult patients in this age group would have the required capacity to provide adequate responses to the topic under investigation; and 2) Parents of children ( $\leq 15$ years) with $S C D$, who attended the SCD clinic or SCA meeting. Parents rather than children were recruited because persons 15 years and under are considered minors and usually cannot take certain important health decisions on their own; they are also most likely to be under the direct care of a parent. Parents of children with SCD younger than 12month were excluded from the study as the pre-testing showed that children in this age group are not likely to experience sufficient significant SCD problems to enable parents to provide useful information for the study.

Purposive sampling with snowballing was used to obtain the samples. I ensured that participants from rural and urban, males and females, different sickle cell genotypes and different ages were represented. Snowballing was particularly useful reaching participants who were not present the clinics or association meetings. Recruitment at the clinics was conducted by the local coordinator and the researcher. Whilst the researcher was still in New Zealand, the local coordinator visited the paediatric SCD clinic on clinic days to explain the research to potential participants. The list of patients and parents who agreed to participate was compiled and sent to the researcher. Recruitment at the SCA (mostly adult patients) was conducted by the researcher. I first contacted the patient's representative for the Association (himself a patient) to approach people who met the inclusion criteria to ask if they would be interested in participating in the study. Once the coordinator provided names, I contacted potential participants by phone to further explain the study. Patients contacted sometimes referred other patients. 
As it was difficult to obtain the required number of participants through phone calls from New Zealand, other participants were recruited when the researcher travelled to Ghana for the fieldwork. The researcher visited the SCA meeting to explain the research to participants and recruited participants that met the inclusion criteria. Most adult participants were recruited through the SCA and patient's networks since the adult SCD clinic had stopped operating. Following the initial recruitment, information sheets and consent forms were given to each participant. Patients who opted to sign the consent form returned them to the local coordinator. Three of the participants signed informed consent forms and 16 gave verbal consent.

Following the initial contact, the researcher phoned each participant to book an appointment. At the appointed time the interview began with a repeat explanation of the research. The actual interviews began with overarching questions "what do you do to care for your sickle cell disease at home?" The remaining part of the interviews were similar to what has been described under health professionals' interviews.

The interview guide was pre-tested among four participants (two parents, two adults) using phone interviews. These participants were recruited through the patient's network. The pre-testing helped to clarify the questions and ensure better flow. It also helped to determine the approximate time for each interview and endurance level of the tape recorder as observed by Babbie [212]. The initial guide consisted of 15 item semi-structured interview guide (Appendix 12) Nevertheless, the questions only served as guide; the aim was not to read them verbatim. Departure from the guide was allowed with the anticipation of obtaining information that had not been previously considered [212]. The interview was mainly conducted in a conversational manner, with the researcher directing the participants where necessary in order to cover important areas for the study.

The data analysis for this stage followed the procedures for analysing deductive CA described in under health professionals' interview. Each of the two datasets (parents, adult) were analysed separately but written together and organised under the subcategories. Under each subcategory, the groups' self-management were compared in terms of commonalities and differences. New variables and concepts were then added to the pool of items to inform the development of the survey for stage 4 . 


\subsubsection{Procedure for maintaining trustworthiness of interviews}

In conducting mixed method research, the researcher is required to use appropriate procedures to ensure that the data, results and interpretation are trustworthy. This involves the use of rigorous procedures for the quantitative strand and persuasive approaches for the qualitative strand [186]. Although similar process are used to achieve trustworthiness for the two traditions, different concepts are applied. Rigour in quantitative research is achieved through checks for validity, reliability, objectivity and generalisability. For qualitative research, trustworthiness is achieved by demonstrating credibility, dependability, confirmability and transferability [210, 215, 216].

In qualitative $\mathrm{CA}$, trustworthiness is effected through the process of credibility, dependability and transferability [211,215]. Credibility concerns the level of accuracy, and confidence or faith that the results of the research depict the phenomenon under investigation $[215,216]$. Credibility can be enhanced through the use of sound research processes including appropriate contexts, participants with varied experience, and use of appropriate data collection methods [215].

Towards this end, a number of steps were taken to ensure that the research process and results were credible and met rigorous standards. Firstly, participants were selected from varied backgrounds including different ages, different genders, rural and urban areas, and people with regular or irregular access to SCD clinic. It can be inferred that the study has included sufficient variety of perspectives as proposed by Graneheim and Lundman [215]. In addition, appropriate data collection methods - in-depth interviews with semi-structured interview guide were used. Moreover, information from participants were audio-recorded and transcribed in the participant's own words, and quotations were extracted from the text to support the researcher's description and interpretation. As recommended by Graneheim and Lundman [215], these measures contribute to ensuring the credibility of information gathered in qualitative CA.

Gillis and Jackson [216] argue that credibility in mixed method studies is achieved when different data sources (documents and people), different methods (observation, interviews, survey), and data types (qualitative and quantitative) are triangulated to answer a single research question as applied in this study. Another approach to ensure credibility is 
consideration of how themes and categories cover the data [215]. In this pursuit, it was ensured that all relevant data were included and irrelevant materials excluded. Efforts were made to ensure that the data was exhaustive and all relevant text were covered by the categories. Credibility was further enhanced through detailed description of the research process to enhance readers understanding of analysis process. Also the description of the results from the categories as advised by Elo and Kyngäs [211] have enhanced credibility.

Other ways of achieving credibility in qualitative CA are through inter-coder agreement, linking results to data, writing the results in sufficient details and attaching appendices to show link between data and results [211, 215]. In this study inter-coder agreement was sought by a review of the coding process by the academic supervisors. In order to link the results to the data, citations from participants were used to support the descriptions and explanations. The research process and tools are also described into sufficient details to enable readers to follow the steps used.

In qualitative CA, dependability refers to stability of the results overtime [215]. In order to ensure dependability Graneheim and Lundman [215] recommend that the categories must be described and conceptually and theoretically grounded, and the results described through the subcategories. As the categories of this study were derived from previous research, theories and frameworks and results were described through themes derived from subcategories, dependability for this study has been enhanced.

Transferability refers to the extent to which the research can be transferred to other settings or groups $[211,215,216]$. As proposed by these authors, transferability in this study was enhanced through accurate description of participants' characteristics. This included a description of the culture, and context, selection and characteristics of the participants, data collection and process of analysis. In addition, the rich and rigorous presentation of findings together with appropriate quotation to support the findings has all enhance transferability [216, 217].

\subsection{Stage 4: The Quantitative Phase}

This quantitative survey constituted the final stage of data collection and analysis of this study. The purpose of the survey was to gather data from a larger cross-section of SCD 
patients and parents in order to provide a better reflection of self-management practices in Ghana, quantify and further explore self-management practices in order to obtain a focused insight of the practices in Ghana, establish similarities and differences in self-management practices among participants by subgroups, and determine the extent to which selfmanagement recommendations and practices identified in the previous qualitative chapters are practiced among a larger sample.

\subsubsection{The survey process}

Surveys are methods of selecting representative samples of a larger population and using the results from the sample to generalise to that larger population $[187,218]$. It is the ideal method for capturing original data from a large population that cannot be directly observed [212]. The method is useful in describing patterns among larger groups rather than in-depth analysis of individual views [219].

The specific design was descriptive cross sectional survey. This type of survey is useful for obtaining data from populations that can be analysed by segments or sections, for example by age or rural versus urban residence. A cross-sectional design was appropriate given the limited time available to complete the data collection, its relative inexpensive cost, and the availability of potential respondents. The more superior methods such as repeated survey or longitudinal studies can take several months or years, are more expensive [216]. The study started with development of the survey using the findings from the literature review and the qualitative phase.

\subsubsection{Instrument development}

In conducting the exploratory sequential mixed method, the researcher decided how the qualitative method would be used to develop instruments for the quantitative phase [187]. For this study, the pool of items identified from the qualitative phase were utilised. In constructing an instrument (the survey), a researcher typically uses the central phenomenon as the construct to be assessed, the broad themes from the qualitative phase as scales to be measured, the individual responses as the variables and specific quotes from individuals as specific items [186]. In line with these recommendations, the survey tool had selfmanagement for SCD as the central phenomenon to be assessed. The subthemes were 
constituted by the categories self-management actions and determinants and subcategories such as nutrition, hydration, and self-medication formed the variables.

Creswell et al. [187] recommends that a researcher using the exploratory sequential approach take the following steps during the physical construction of the instruments: determining what to measure, generating an item pool, determining scales of measurement, reviewing items by experts, consider including validated instruments, validating the instrument and evaluating the items. In this study the questionnaire construction process began with an identification of a pool of items which were derived from the literature review and qualitative studies reported in the previous chapters. Thereafter, the self-management actions and determinants were set out as the section titles and the pool of items organised under these sections. The questions were then reviewed and refined to reflect their relevance under the sections. Scales of measurements were then attached to each of the questions.

The draft instrument was then reviewed and refined several times by the researcher, academic supervisors and statistician to ensure that they were precise and measured the intended purpose. Once the construction was completed, all questions stems were translated to the commonest local language in Ghana (Twi) by one of the SCD experts in Ghana and the researcher.

The final survey tool consisted of a 47 item questionnaire (Appendix 14). Questionnaires are the most ideal instrument for survey research as they help to capture original data from a large population [212, 220]. In conducting a survey the researcher must choose appropriate question forms may be open or closed ended [212]. Close questions are more suitable for questionnaires because these provide greater uniformity of responses and are more easily processed than open questions. Open questions are more susceptible to bias as the researcher has to interpret and code before they can be processed by a computer [212]. The questionnaire had 24 closed questions only, 12 closed questions with open ended sub questions, and 11 open ended questions only.

The 36 closed questions included nominal, ordinal, dichotomous and ranked responses. As the major concepts being measured such as self-monitoring, and self-diagnoses do not have single measures, composite measure in the form of frequency scales were used to report the frequency of specific actions. As noted by Babbie [212] scales are composite 
measure that enable a researcher to manipulate and summarise several data items simultaneously using single scores whilst at the same time maintaining the information from all of the individual indicators. In addition, a Likert type scale was adopted from the Chronic Disease Self-Management Programme [221] (no permission required) to rate self-treatment actions, health outcomes (perceived health status, quality of life), and knowledge of SCD care. Questions with the same answer categories were constructed in matrix forms to save space and facilitate comparability of responses by individual respondents.

As closed questions can restrict respondents' options and may not capture unanticipated answers [212], open questions were included as sub-questions under selected closed questions to further explore respondent's perspectives. Although most questions were directed to all participants, some were not applicable to everyone. In this case the instrument clearly instructed the respondents to proceed to next applicable questions. The final nstrument was set out in six sections as follows:

- Section 1: Socio-demographic and clinical characteristics of respondents

- Section 2: Self-management action

- Section 3: Evaluation of self-treatment actions

- Section 4: Evaluation of health outcomes

- Section 5: Self-management resources

- Section 6: Key challenges in managing SCD

Section 1 comprised of the socio-demographic and clinical characteristics of participants such as age, sex, educational attainment, occupation, type of SCD, complications experienced, proximity of SCD clinic to respondent's residence, proportion of income spent in managing SCD, and registration status with the national health insurance scheme. Part of section one was put at the end of the questionnaire. Section 2 was concerned with selfmanagement actions including: preventive health and maintenance, self-monitoring, selfdiagnosis, and self-treatment. Section 3 focused on evaluation of self-treatment actions. Section 4 focused on identifying health outcomes in terms of self-rated health, and quality of life. Section 5 concerns self-management resources people possess such as material and medicinal resources, knowledge of SCD, and level of social support. Finally, Section 6 was concerned with key challenges people encountered in caring for SCD. 
Tailored survey instruments were developed for each group (adults/ parents) with similar content except for few questions. Items including vaccination, hand-foot-syndrome, and observation of behaviour were specific for the parents' questionnaire, whilst items on "listening to body" was also included in the adult questionnaire. The sections began with instruction for completion. As observed by Babbie [212] providing instructions help clarify the questions for the respondents and reduces confusion.

Validating the instrument was pursued through expert review and pre-testing among a cross-section of patients and parents. The instrument was reviewed for face validity by the University statistician and academic supervisors, then for content validity by three sickle cell experts in Ghana. The SCD experts were asked to check whether the questionnaires had the appropriate items to measure self-management for SCD patients and whether they had a sufficient number of items. In addition, the experts were asked to assess whether the questions were simple, concise, readable, comprehensive and of appropriate length. Content validity was met as per the reviewer's comments; all three reviewers agreed that the questions covered key areas for self-management for SCD and were important to ask. They advised that the selfmanagement actions questions be brought to the beginning whilst the questions on sociodemographic characteristics be asked last. They also agreed that the wording of the survey was enough to ensure anonymity, and that they were readable, and comprehensive.

The instrument was then reviewed by 10 non-medical people with low to moderate educational background in Ghana for their level of comprehensiveness, and precision. The instrument was revised again based on comments from these people and presented back to the statistician for final validity check. Finally the questions were pre-tested among 12 respondents ( 6 adults, 6 parents) at a monthly meeting of the SCA (Ashanti regional branch). The pre-testing resulted in modifications removal, additions, and re-arrangement of questions to reflect a better flow of the final instrument (Appendix 13).

\subsubsection{Conducting the survey}

The following steps were taken to recruit participants, administer the survey and analyse the data. An estimated sample size of 422 was planned for the study. This was calculated with the survey calculator [222] using a 5\% margin of error, 95\% confidence level 
[154], an estimated SCD population of 400,000 in Ghana [59] and a 10\% non-response rate [223].

The consecutive sampling procedure was used to select respondents. In this method, all subjects who meet the inclusion criteria are selected until the required sample is obtained or the survey period is over [224]. This method is relatively easy to use, and less likely to be manipulated by the researcher. However, the method is not based on randomisation and may be affected by variations in the type of persons who seek health services at different times of the year. This disadvantage was unlikely to have affected the study given that no evidence exists to indicate that SCD clinic attendance has seasonal variations in Ghana. Furthermore, all accessible SCD patients in the study region within the data collection period were targeted for sampling.

Consecutive sampling was the most feasible method given the challenges the researcher encountered in the field that made it impossible to use methods that involved randomisation. Firstly, the data collection period which was scheduled for six months was reduced to three months because the researcher was required by Victoria University to delay the field work in view of the Ebola epidemic in the West African sub-region. Secondly, five out of the seven sickle cell clinics initially targeted for sample selection had stopped operating at the time of data collection limiting the study sites to two - one city and one district hospital. Thirdly, the city clinic that previously saw both adults and children had stopped seeing adult patients (following completion of the new-born screening programme). This weekly clinic saw only paediatric patients up to 12 years of age. Fourthly, the district clinics that operated weekly had an average attendance of five patients. Given the above scenario, engaging in randomisation would have resulted in low numbers of participants in the time available to me.

Three additional strategies were used to maximise data collection given the field challenges. Firstly, six research assistants were trained to assist with data collection. Secondly, I gained permission from the medical director to collect the phone numbers of patients on the register from two SCD clinics that had stopped operating and followed these patients and parents up through phone calls for recruitment. I also recruited adult SCD patients from the adult medicine specialist unit at the Komfo Anokye Teaching Hospital. All 
survey respondents were recruited when the researcher travelled to Ghana for the fieldwork. As part of the recruitment process, I visited the functioning clinics on the clinic days to explain the research and recruited patients and parents who were eligible.

A survey may be administered through self-administration, face-to-face encounter, by phone, or internet [212]. This survey was mostly delivered by face-to-face and over the telephone as many respondents had low literacy levels and could not complete the questionnaires on their own. Respondents who were surveyed by telephone could either not be interviewed at the clinic or were recruited from the closed clinics. Where respondents selfcompleted the survey, on the spot checks were conducted to ensure that they were fully completed [212, 214]. Completed questionnaires were organised and collated based on adults and parents categories.

\subsubsection{Data analysis}

Quantitative data analysis includes the process of data preparation, statistical analysis, and reporting the findings [186, 225]. Data preparation involves checking, editing, coding, assembling and transforming. Checking involves decisions about usability of the completed survey. A survey may be unusable if an unacceptable number of questions remain unanswered, it has missing pages, an unintended person has answered a question, questions are inappropriately answered, or the question is received too late to include in the analysis $[212,225]$. Eleven surveys, representing $3 \%$ of 324 of completed surveys were discarded as they contained too many unanswered spaces, or had missing pages. All discarded questionnaires were self-administered ( 7 adults, 4 children). In relation to the large number of usable questionnaires ( $n=313$ ), the discarded data was not large enough (up to 5\%) to adversely affect the results as indicated by Kent [225].

Editing involves verifying response consistency, accuracy making necessary correction and deciding whether some or all parts of a questionnaire should be discarded [225]. The process involves logical, range, and response set checks. Logical checks were conducted to check the consistency with which respondents answered the question. For example if a respondent answered "NO" to a question unintended for some participants, I check to verify if the respondents has skipped the appropriate questions to the next intended 
questions. Responses that failed logical checks were corrected by the researcher. The range of responses were inspected for each question, and all responses were valid.

Coding involves reducing information into a more limited set of attributes [212]. Since SPSS was used for the analysis, I ensured that numerical values were assigned for qualitative options (binary, dichotomous and nominal values). Higher values were assigned to higher or most positive responses for ranked ordinal responses. All continuous variables were entered directly into SPSS (e.g. age, number of times a person exposed crisis). Qualitative data were analysed both as text and also coded quantitatively for descriptive analysis. New variables (binary or nominal) were created from open responses including "other" options. In order to preserve the richness of the data, some qualitative information are presented in the narrative to support the quantitative results. Unanswered questions were designated "not applicable (NA)."

Data were assembled by direct entry into the Statistical Package of Social Science (SPSS) data matrix created by the researcher (IBM SPSS Statistics 22). The two datasets (adults, parents) were joined for data analysis. Following the data entry, a variety of data transformation was carried out to facilitate the analysis. Class intervals were created where continuous values were organised into categorical groups such as age groups, number of times attending SCD clinic, number of crises experienced. Totals were also computed from researcher-selected items (pre-determined self-management actions) to obtain the total number self-management actions performed by each participant. This figure was then used to compute descriptive statistics for self-management and comparison among sub-groups. All scale items were regrouped into fewer categories; for example, predetermined preventive health actions which were based seven category frequency rating scales were regrouped into two categories (regular and periodic use). The seven item Likert type scale and 10 item numerical scale used to measure health status and QOL were regrouped to two categories. For example, health status was regrouped as "good or better" and "poor or fair." The responses for good, very good, and excellent were grouped as "good or better" whilst responses for fair or poor remained unchanged. Non-committal responses such as "don't know", "neither”, or "undecided" were either considered as equal to missing, "don't know", or excluded from the analysis depending on the question. Where these were included in the analysis, the purpose was to demonstrate a lack (or absence) of the attribute being investigated. For instance people 
who said they did nothing in managing certain conditions were sometimes included in the analysis to show their inability or lack of knowledge in self-management.

The aim of the statistical analysis was to describe participant's self-management actions and determinant variables; establish the differences and similarities between adults and children's self-management actions, establish the relationship between self-management actions and participants socio-demographic characteristics such as urban versus rural residents and gender. Descriptive statistics (measures of central tendency and of dispersion) were used to describe respondent's background. In order to test the difference between sub-groups on outcome variables, inferential statistics were conducted. The chi-square test for independence and goodness of fit were conducted where appropriate for categorical variables, and the independent samples t-test or one way ANOVA for continuous variables [226]. When chisquare test of independence were applied to $2 \times 2$ (two roles, two columns) tables the Yate's Continuity Correction has been applied as this give a better approximation of the theoretical sampling distribution of the chi-square. As recommended by Rovai et al. [226] chi square tests were not conducted for $2 \times 2$ if one cell has frequency count of less than 5 .

Normality was tested for self-management actions using the Shapiro-Wilk test [226]. The results showed that self-management actions were approximately normally distributed for all independent variables ( $>0.05)$. Furthermore, the assumption for homogeneity of variance was tested when t-tests and one-way ANOVA were used, and was found to be met for all variables as assessed using Levene's test. Due to the ordinal nature of the data, Spearman's rank correlation was used to test the relationship between self-management actions and patients health outcomes (health status and quality of life); and possession of some selfmanagement resources.

\subsection{Ethical Considerations}

Researchers investigating human experiences are required to follow ethical standards which imply a generally acceptable way of conducting scientific enquiries. Researchers owe these ethical responsibilities to their human subjects and the scientific community $[212,226]$. In protecting the human subjects, researchers are expected to ensure that participation in the study is voluntary such that there is no coercion to participants. Furthermore, a study must not cause physical or psychological harm to subjects $[186,212]$. In line with these expectation, 
steps were taken to ensure that the rights, needs, values, and desires of participants were protected in all segments of the study by seeking the appropriate permissions from institutions, subjects and significant others [226].

The study proposal was reviewed and approved by Victoria University of Wellington and two health institutions - Ghana Health Service, and Komfo Anokye Teaching Hospital (KATH) (Appendices 1-7). Once the first phase of the study was completed, a progress report and the completed survey instrument were sent to the three institutional review boards for approval. A request for amendment were sought and approved by all three institutions when there was the change in data collection method for the second and third qualitative phase from face-to-face to telephone interviews. This change was required due to the delay in data collection because of the Ebola situation in West African. Following institutional review approvals, permission was sought from the Regional Health Authorities at the study region and the Executive Committee of the Sickle Cell Association of Ghana for access to participants (Appendix 8).

Thereafter, strategies were implemented to ensure respect and care of participants including voluntary participant as well as protection from physical and psychological harm. These were formalised in participants' information sheets (Appendix 9) and informed consent forms which outlined the information about the nature of the research and steps taken to ensure confidentiality, and voluntary participation. All interviewed participants were given copies of the information sheet and consent form (Appendices $10 \& 11$ ). For the survey, consent was assumed if the respondents completed and returned the survey.

These steps assured participation in the research was voluntary and patients had the liberty to withdraw their participation from the study up to two weeks after data collection. Participants could decline to answer any question. The process of confidentiality was used to protect participants from harm. For example those who assisted with recruitment and completing questionnaires agreed to protect the confidentiality of potential participants and respondents. Names and other identifying information were excluded from data collection tools. During analysis, any quotations that could potentially identify a participant were not used directly. Written and digital records for the interviews were kept under "lock and key" in a secure area at the university. Analysed data and other electronic information were kept on 
the computer hard drive and password protected. Finally, as recommended by Babbie [212]. I ensured that the data, analysis, reportage of results, limitations, shortcomings, negative and unexpected findings were accurately recorded. The next four chapters report the findings from the study. 


\section{Chapter 6: Results of Website Review}

This chapter presents the results of the content analysis of SCD focused websites. The results are organised in two sections. The first section presents an overview of the websites, their sources and physical characteristics. The second section presents the self-management recommendations derived from the sites.

\subsection{Overview of Websites, Sources and Characteristics}

Fourteen websites from five countries met the inclusion criteria and were included in the analysis. The websites were from North America-USA [9], Canada [2], Europe-Norway [1], UK [1], and Africa-Uganda [1]. No websites from Ghana met the inclusion criteria. Examination of the characteristics of the websites identified three with search options and five provided an interactive platform which was usually Facebook and Twitter. Sources of websites are referred to by abbreviations such as US1, UG (Table 8).

Thirty-three relevant Information Education and Communication Materials (Materials) were identified from the 14 websites. Of these, web pages comprised 20 (61\%), audio-visual 7 (21\%) and books and printable documents $6(18 \%)$. The USA accounted for $55 \%$ of the materials. All seven videos were located at the site of Sickle Cell Association of Ontario, Canada. None of the websites provided all types of materials (Table 9).

Almost all the IECMs targeted more than one audience. Half of these materials provided for patients only or both patients and parents. Other target groups included the public, teachers, employers and health professionals (Table 10). Four materials that targeted patients only were found in four different websites-USA (2), UK (1) and Norway (1). Eleven materials that targeted patients and parents only were retrieved from four websites (Canada [1], USA [2] and Uganda [1]). The five websites that targeted patients and the public were from Canada (2) and USA (3). The remainder which targeted all the audiences were from the USA. The USA websites had more comprehensive and many targeted audiences than the sites from the other countries. 
TABLE 8. WEBSITES CHARACTERISTICS

\begin{tabular}{|c|c|c|c|c|}
\hline Organisation/Country & $\begin{array}{l}\text { Search/ } \\
\text { Interactivity }\end{array}$ & URL & $\begin{array}{l}\text { Website } \\
\text { code }\end{array}$ & $\begin{array}{l}\text { Websites } \\
\text { ID }\end{array}$ \\
\hline $\begin{array}{l}\text { Sickle Cell Association } \\
\text { Ontario (Canada) }\end{array}$ & $\begin{array}{l}\text { Search } \\
\text { Facebook } \\
\text { Twitter/In }\end{array}$ & $\begin{array}{l}\text { http://www.sicklecellontario.org/ } \\
\text { AboutSickleCell/HealthyLiving.a } \\
\text { spx }\end{array}$ & 1.0 & CA1 \\
\hline $\begin{array}{l}\text { Sickle Cell Association } \\
\text { Hillsborough County (USA) }\end{array}$ & $\begin{array}{l}\text { Search } \\
\text { Facebook }\end{array}$ & $\begin{array}{l}\text { http://scahillsborough.org/treatme } \\
\text { nts/ }\end{array}$ & 2.0 & US1 \\
\hline $\begin{array}{l}\text { Sickle Cell Information } \\
\text { Centre (USA) }\end{array}$ & & https://scinfo.org/ & 3.0 & US2 \\
\hline $\begin{array}{l}\text { Sickle Cell Association of } \\
\text { Uganda (Uganda) }\end{array}$ & E-mail & $\begin{array}{l}\text { http://www.sicklecelluganda.org/ } \\
\text { home-care-management.html }\end{array}$ & 4.0 & UG \\
\hline Sickle Cell Society (UK) & - & $\begin{array}{l}\text { http://www.sicklecellsociety.org/ } \\
\text { websites/123reg/LinuxPackage22/ } \\
\text { si/ck/le/sicklecellsociety.org/publi } \\
\text { c html/resources/view/9 }\end{array}$ & 5.0 & UK \\
\hline $\begin{array}{l}\text { Sickle Cell Association } \\
\text { National Capital Area } \\
\text { (USA) }\end{array}$ & $\begin{array}{l}\text { Facebook } \\
\text { Twitter }\end{array}$ & http://www.scancainc.org/ & 6.0 & US3 \\
\hline $\begin{array}{l}\text { Sickle Cell Association } \\
\text { Canada (Canada) }\end{array}$ & $\begin{array}{l}\text { Facebook } \\
\text { Twitter } \\
\text { E-mail }\end{array}$ & http://www.sicklecelldisease.ca/ & 7.0 & CA2 \\
\hline $\begin{array}{l}\text { Norwegian Sickle Cell } \\
\text { Association (Norway) }\end{array}$ & - & http://nscao.org/english/ & 8.0 & NW \\
\hline $\begin{array}{l}\text { Sickle Cell Foundation } \\
\text { Atlanta (USA) }\end{array}$ & - & $\begin{array}{l}\text { http://sicklecellga.org/overview- } \\
\text { video/ }\end{array}$ & 9.0 & US4 \\
\hline $\begin{array}{l}\text { Children's Sickle Cell } \\
\text { Foundation (USA) }\end{array}$ & & https://www.cscfkids.org/ & 10.0 & US5 \\
\hline $\begin{array}{l}\text { St Lucia Sickle Cell } \\
\text { Association (USA) }\end{array}$ & & $\begin{array}{l}\text { http://stluciasicklecell.weebly.co } \\
\text { m/index.html }\end{array}$ & 11.0 & US6 \\
\hline $\begin{array}{l}\text { Net Care International } \\
\text { Resource for caregivers } \\
\text { (USA) }\end{array}$ & Search & $\begin{array}{l}\text { http://www.netofcare.org/content/ } \\
\text { specific illnesses/sickle_cell.asp\# } \\
\text { treatment }\end{array}$ & 12.0 & US7 \\
\hline $\begin{array}{l}\text { Sickle Cell Association } \\
\text { Peninsula (USA) }\end{array}$ & Facebook & http://www.orgsites.com/va/pasca & 13.0 & US8 \\
\hline $\begin{array}{l}\text { Centre for Disease Control } \\
\text { and Prevention (USA) }\end{array}$ & $\begin{array}{l}\text { E-mail } \\
\text { Twitter } \\
\text { Facebook } \\
\text { YouTube }\end{array}$ & $\begin{array}{l}\text { https://www.cdc.gov/ncbddd/sickl } \\
\text { ecell/index.html }\end{array}$ & 14.0 & US9 \\
\hline
\end{tabular}


TABLE 9. DISTRIBUTION OF MATERIALS AND TYPES BY COUNTRY OF WEBSITE

\begin{tabular}{|l|r|r|r|r|r|}
\hline Country & Web pages & $\begin{array}{r}\text { Audio- } \\
\text { visual }\end{array}$ & Books & Print & Total (\%) \\
\hline USA & 14 & - & 1 & 3 & $18(54.5)$ \\
\hline Canada & 3 & 7 & - & - & $10(30.3)$ \\
\hline Norway & 2 & - & - & - & $26.1)$ \\
\hline UK & - & - & 1 & 1 & $2(6.1)$ \\
\hline Uganda & 1 & - & - & - & $1(3.0)$ \\
\hline Total & $20(60.0 \%)$ & $7(21.0 \%)$ & $2(6.1 \%)$ & $4(12.1 \%)$ & $33(100.0 \%)$ \\
\hline
\end{tabular}

TABLE 10. TARGET AUDIENCE AND AUTHORS' BACKGROUND OF MATERIALS BY WEBSITE

\begin{tabular}{|l|r|r|}
\hline \multicolumn{1}{|c|}{ Category } & $\begin{array}{r}\text { Frequency (\%) } \\
\mathbf{n = 3 3}\end{array}$ & No. Websites \\
\hline Target audience & $8(24.2)$ & 4 \\
Patients only & $11(33.3)$ & 4 \\
Patients, parents & $6(18.2)$ & 5 \\
Patients, public & $2(6.1)$ & 2 \\
Patients, parents, public & $1(3.0)$ & 1 \\
Parents, teachers & $1(3.0)$ & 1 \\
Parents, teachers, employers & $3(9.0)$ & 1 \\
Parents, care providers & $1(3)$ & 1 \\
Patients, parents, care providers & & 1 \\
& & \\
Author's background & $7(21)$ & \\
Health professionals & $26(79)$ & 1 \\
Not specified & & \\
\hline & & \\
Types of references & $32(97)$ & \\
Primary and secondary & & \\
Not named & & \\
\hline
\end{tabular}

\subsection{Self-Management Recommendations}

The messages on self-management recommendations were classified under two main categories self-management actions and self-management determinants. Overall, 371 messages were located for both categories. Majority of the messages related to selfmanagement actions $(317,85.4 \%)$ and included preventative health behaviour $(229,72.2 \%)$, self-monitoring (17, 5\%), self-diagnoses (27, 9\%), self-treatment (44, 13.9\%) (Table 11). 
Messages on self-management resources accounted for the highest (62.7\%) in the selfmanagement determinants category.

TABLE 11. REFERENCES TO SELF-MANAGEMENT ACTIONS AND DETERMINANTS

\begin{tabular}{|l|r|}
\hline No. of Variables & $\begin{array}{r}\text { No. }(\boldsymbol{\%}) \\
\mathbf{n = 3 7 1}\end{array}$ \\
\hline Self-management action $(\mathbf{n}=\mathbf{3 1 7}, \mathbf{8 5 . 4} \%)$ & $229(72.2)$ \\
Preventive health & $44(13.9)$ \\
Self-treatment & $27(8.5)$ \\
Self diagnoses & $17(5.4)$ \\
Self-monitoring & \\
Self-management determinants $(\mathbf{n = 5 4 , 1 4 . 6 \% )}$ & \\
Self-management resources & $34(63.0)$ \\
Contextual factors & $11(20.4)$ \\
Vulnerability factors & $9(16.7)$ \\
\hline
\end{tabular}

The subsequent subsections describe the results of self-management actions and determinants. Under each part, quantitative results are presented first presented to describe frequency of messages and number of websites that provided the recommendations. Of note is that some self-management determinates (school teacher management, general knowledge of SCD, pregnancy precaution) are weaved through self-management actions to correspond to the context within which the variables were located. The remaining self-management determinants (avoidance of overprotection, social support, psychological coping, vocational and employment selection) were described within the context psychosocial context and have been described as such.

\subsection{Self-management Actions}

This section presents the results of self-management actions including preventive health, self-monitoring, self-diagnosis and self-treatment.

\subsubsection{Preventive Health (Quantitative Results)}

Thirty-one areas of preventive health and maintenance recommendations were identified. These were organised as routine medicines; medicines, food, substances that must be avoided; non-medicinal actions; and consultation with health professionals (Table 12). 


\begin{tabular}{|c|c|c|}
\hline Variable & $\begin{array}{c}\text { No. }(\%) \\
n=142\end{array}$ & $\begin{array}{c}\text { Website } \\
n=14\end{array}$ \\
\hline Routine medicines $(\mathrm{n}=36,15.7 \%)$ & & \\
\hline Folic acid supplementation & $12(5.2)$ & 5 \\
\hline Penicillin prophylaxis & $14(6.1)$ & 4 \\
\hline Malaria prophylaxis & $3(1.3)$ & 3 \\
\hline Other supplements & $3(1.3)$ & 2 \\
\hline Zinc supplementation & $2(0.9)$ & 1 \\
\hline CAM/Herbal & $1(0.4)$ & 1 \\
\hline Iron removing medication & $1(0.4)$ & 1 \\
\hline Medications/food/substances to be avoided $(n=29,12.7 \%)$ & & \\
\hline Iron containing medicines & $5(2.2)$ & 4 \\
\hline Cigarette smoking & $5(2.2)$ & 4 \\
\hline Alcohol & $5(2.2)$ & 4 \\
\hline Diuretics medications & $4(1.7)$ & 2 \\
\hline Use of hard drugs & $3(1.3)$ & 1 \\
\hline High sodium food & $2(0.9)$ & 1 \\
\hline Decongestants & $1(0.4)$ & 1 \\
\hline Aspirin/ salicylates & $1(0.4)$ & 1 \\
\hline Citrus foods/ medicines & $1(0.4)$ & 1 \\
\hline Coffee & $1(0.4)$ & 1 \\
\hline Diet soda & $1(0.4)$ & 1 \\
\hline Non-medicinal $(142,62.0 \%)$ & & \\
\hline Adequate hydration & $38(16.6)$ & 13 \\
\hline Activity limit & $25(10.9)$ & 8 \\
\hline Avoidance of temperature & $25(10.9)$ & 7 \\
\hline Vaccination promotion & $14(6.1)$ & 8 \\
\hline Adequate nutrition & $9(3.9)$ & 7 \\
\hline Avoidance of low $\mathrm{O}_{2}$ environment & $8(3.5)$ & 4 \\
\hline Travel precaution & $8(3.5)$ & 3 \\
\hline Hygiene measures & $8(3.5)$ & 4 \\
\hline Psychological stresses & $4(1.7)$ & 3 \\
\hline Adequate rest & $2(0.9)$ & 2 \\
\hline Malaria prevention & $1(0.4)$ & 1 \\
\hline Consult health professionals $(\mathrm{n}=22,9.6 \%)$ & & \\
\hline Adherence to medical treatment & $19(86.4)$ & 11 \\
\hline Regular follow up & $3(13.6)$ & 3 \\
\hline
\end{tabular}




\section{Preventive Health (Qualitative Results)}

The websites provided wide range of advice regarding preventive health. The focus of the advice was to enable the patient to keep well and remain in a stable condition. The advice tended to fall into four thematic categories. The first category urged patients to perform health promotional and body protective activities to maintain physiological balance. The second category advised patients to modify their behaviours to avoid triggers of disease exacerbations which may be internal or external to the patient. The third category involved interactions with health professionals. The fourth category concerned the SCD child at school.

Regarding routine medicines, more websites referred to folic acid supplementation and penicillin prophylaxis (26 of 36 references). These references were located in a Canadian, US and UK websites. Three Websites from Uganda, UK and Norway referred to use of malaria prophylaxis in malaria endemic countries. The only recommendations for the use of herbal medicine (pear leaves and fresh beet root) as preventive treatment were located in the Ugandan website. Two websites from the USA and Norway recommended use of other supplements including L-arginine supplementation, Blackstrap Molasses and Diascovite.

On medicines and substances that must be avoided, more messages related to ironcontaining medicines, cigarette smoking, and alcohol consumption ( 5 of 22 messages each). These were located at websites from all five countries. The remaining references were mostly cited by websites from the USA, Canada and Norway. Diuretics were the other commonest medicines patients were advised to avoid. Substances including alcohol, high sodium foods, coffee and dietary soda were also mentioned in connection with their ability to cause increased diuresis, dehydration and painful crisis.

With respect to non-medicinal actions, all websites except the Sickle Cell Association of Canada recommended hydration (38 of 142 messages) followed by limitation of activities (25 of 142). Recommendations regarding avoidance of low oxygen tension and travel precautions were located at websites in USA, Canada and the UK only. Recommendations for pneumococcal vaccination were located at websites from all the countries apart from the Ugandan site. The majority of the references (15 of 19) for adherence to medical treatment were from the USA. The remaining were from Norway (1), Canada (1), UK (1) and Uganda (1). All three references regarding medical follow-up were from the USA. 


\subsubsection{Health Promotion and Body Protective Recommendations}

A wide varieties of health promotional and body protective advice were provided including measures that does not involved the use of medicines (non-medicinal actions) and those involving use of medicines (medicinal actions). The non-medicinal health promotional advice included adequate hydration, adequate nutrition and personal hygiene. Hydration and nutrition were the commonest. Medicines recommended for health promotion were supportive or protective in nature and folic and penicillin prophylaxis were the commonest.

As indicated in Table 12 messages on hydration were the most recommended. The websites consistently promoted hydration as an efficacious measure for the general wellbeing for SCD patients. Phrases such as "lots of water," "drink plenty," "drink frequently," "higher than normal fluids," and "well hydrated" were used to describe the need for SCD patients to drink more than people without SCD. The sites recommended hydration for both adults and children. These messages tended to centre on the rationale for hydration, the types of fluids recommended, daily requirements, and conditions under which fluid intake should be increased.

The commonest rationale for hydration was to prevent dehydration. Other reasons were that that hydration could prevent pain, infection and fatigue. Most recommendations were accompanied by an explanation of the pathophysiological mechanism including the role of hydration in the kidney, red blood cell function and the implication for sickling. Water was the most recommended the fluid of choice. Some sites recommended other fluids including "soft drinks", "fruit juice”, and "sodas" (US2) and Oral Rehydration Salt (UG).

Six sites (CA1, US2, US3, US5, UG, NW), specified the daily requirements for water but there were variations in units of measurements. The Canada and USA sites expressed requirements in the number of glasses per day (8-10 for adults and 4 for children). The Norwegian site expressed this in relation to body weight and litres.

Your body must replace 2-3 quarts of water every day. As a rule of thumb, for every 15lbs of weight, you need 8 ounces (1 cup) of water...Patients must drink lots of fluids daily: 3 litres at least for children and 5 for adults. (NW) 
Messages also addressed mode of drinking including the need to drink small volumes frequently throughout the day as opposed to large quantities infrequently, planning to drink and accessing equipment to support drinking. Other messages advised on conditions under which fluid intake needs to increase including exposure to the extended duration of sunlight, cold weather, swimming, and air travel. Sites from the USA and UK cautioned parents regarding increased urination and possible bedwetting among children and children's need for frequent use of the bathroom.

Nutrition related messages were the second commonest non-medicinal health promotional recommendations. Messages recommended that SCD patients consume healthy diets with balanced selections of food, and growing children are provided additional calories in addition to their three meals a day to avoid anaemia. For SCD children experiencing prolonged tiredness, parents and caregivers were advised to consult health workers for nutritional support (US2). A recommendation from a US site was that food rich in fish oil (Omega N3 Fatty acid) can prevent the occurrence of a crisis

Few sites advised patients on hygiene measures in order to prevent infection. The advice included hand hygiene, food hygiene, avoidance of potential reservoirs of infection, and prompt treatment of infection. Regarding hand hygiene, patients were advised to wash their hands frequently with soap and water or with alcohol-based hand cleansers before food preparation and eating, and after eating or using the toilet. Food hygiene recommendations included washing utensils, washing surfaces that come into contact with raw food, and washing fruit and raw foods:

Avoid food bacteria - certain uncooked foods can carry harmful bacteria. Be sure to wash anything that comes into contact with contaminated food including hands, cutting boards, knives, counters, or any other utensil. Wash all fruits and vegetables before eating them and cook meat until its well-done. (US5)

One USA site (US8) advised people to ensure prompt treatment of infections when these occur. Patients were also advised to avoid potential reservoirs of infection such as uncooked food, reptiles, garbage and contact with people with cold and flu. 
The other areas of health promotional messages concerned use of supportive medicines. The sites provided advice on certain medicines patients must endeavour to take for supportive or protective care. These supportive medicines were folic acid, other vitamin supplements, and herbal medicines whereas the protective medicines were penicillin prophylaxis and vaccinations. Folic acid was the commonest supportive medicine and was mostly recommended to maintain erythropoiesis: CA1 also included that folic acid helps to prevent bacterial infection. Use of folic acid supplements, rather than dietary sources, was almost universally promoted as the latter is usually destroyed through cooking.

Leafy vegetables are a good source of folic acid, however, cooking them will reduce the amount of this vitamin... Therefore my opinion is that folic acid may help people with sickle cell, can't hurt, and is quite simple to take. (CA1)

All messages recommended daily supplement as the body does not store folic acid. Dosage in the form of "pills" was mostly recommended but there were variations in the recommended dosage. US2 recommended $1 \mathrm{mg} /$ day irrespective of age and body size. NW recommended 400 micrograms $(\mathrm{mg}$ ) for adults and $600 \mathrm{mg}$ for pregnant women, and supplements are started from initial diagnosis of SCD regardless of age and genotype.

Two sites (CA1, NW) recommended other supplements including zinc as patients naturally have a zinc deficiency and supplementation promotes the growth of young people and boosts immunity. Other supplements recommended by the sites included L-arginine, black strap molasses, Diascovite, and vitamin C to boost immunity.

Penicillin prophylaxis was the commonest recommended preventive medication to forestall infection, especially with pneumococcus. Different sites recommended different ages of commencement and completion. CA1 specified that penicillin should commence at two months of age. A site (US2) did not specify the age of commencement but recommended penicillin for all newly diagnosed SCD patients.

Three sites described ages (range 4-6 years) at which the prophylaxes must be completed. The CA1 site specified penicillin must be stopped after the commencement of vaccination. The UK indicated that parents should discuss continued penicillin use with their healthcare workers after six years of age. Most sites did not state the dosage;US2 site 
recommended $125 \mathrm{mg}$ twice a day up to 6 years and $250 \mathrm{mg}$ twice a day beyond six years when necessary. All messages indicated a daily dose and US2 recommended a twice-a-day schedule for patients.

Few sites recommended vaccination to protect SCD patients' health. In addition to standard vaccination for children, US5 and CA1 advised patients to ensure annual influenza and pneumococcus vaccinations. Although most messages did not specific the targeted age groups, there were more messages recommending pneumococcus vaccination for children than adults.

People with sickle cell disease, especially infants and children, are more at risk for harmful infections. Pneumonia is a leading cause of death in infants and young children with sickle cell disease. Vaccinations can protect against harmful infections. (US8)

\subsubsection{Behaviour Modification to Avoid Triggers of Disease Exacerbation}

The websites offered a range of advice regarding avoidance of behaviours that can directly trigger disease exacerbation. These behaviours also involve the use of non-medicinal and medicinal actions. The commonest non-medicinal actions were avoidance of overactivity, and extremes of temperature. The others concerned avoidance of low oxygen environment, and psychological stresses. Another aspect of these advice concerned precautions regarding air travel. The sites also advised patients to avoid use of certain medicines, food and beverage that can trigger crisis.

Activity related messages were described within the context of physical activities engagement, cautions on over activity, and measures to mitigate the effect of over activity. Twenty-five recommendations from eight sites encouraged SCD patients to engage in physical activities such as play, sport, gym and exercise to improve circulation and reduce blockage of blood vessels. Despite these benefits, seven sites cautioned patients about over activity. Three sites (CA1, UK, and US2) linked over activity to the possible painful crisis and urged SCD patients to be aware.

The Norwegian site recommended breathlessness as an indication for determining the point of over activity with other messages indicating patients subjectively determine their own 
activity limit and avoid pushing themselves beyond these limits. US3 cautioned that patients must ensure that there was no contraindication for them to engage in purposeful physical activities. The Norwegian site also advised patients to consult their physician before engaging in any exercise programme.

There were three sets of advice to mitigate the effect of over activities. The first concerned situations where patients engage in exercise programmes. Patients were advised to drink extra fluids, set their own pace, and take breaks and rest when tired. The second set related to routine activities of daily living. Patients were advised to ensure adequate rest by having flexible daily schedules, access to transportation and resting places for school children. The third set recommended rest and activity as part of complementary actions that patients should routinely pursue together to stay healthy: "The best success by our patients is found by staying hydrated with water, well-balanced nutrition, no smoking or use of street drugs, and not over exerting" (US2).

Regarding extremes of temperature, the sites advised patients to avoid extreme weather conditions, cold temperatures and exposure to rain. Potential causes of cold temperatures indicated by the sites were swimming in non-heated pools, cold baths, doing laundry, getting caught in rain, and cold drinks from refrigerators. These sites were mostly located in USA and UK sites. Many other sites advice on avoidance of too hot or too cold temperatures without mentioning specific conditions. Recommended strategies to cope with extremes of temperature included dressing for the weather, controlling internal environmental temperature, using warm water and keeping warm and dry. "The keyword for preventing crisis are warmth and dry...Make sure sickle cell child is always warm and dry. The condition may get worse in the cold or wet" (UK).

The sites also cautioned patients to avoid areas and activities that result in low oxygen tension. The advice focused on precipitating conditions, and mitigation strategies to reduce the effects of low oxygen tension. Commonly mentioned precipitating factors were travelling in unpressured aircraft, mountain climbing, scuba diving, general anaesthesia and sporting activities. The sites advised people to avoid these activities or in default use mitigation strategies to offset their impact. Recommended mitigation strategies concerned use of supplemental oxygen during air travel. The US2 site specified the dosage of oxygen required 
during air travel: "We generally recommend 2 litres/minute but this is not based on direct measurements of haemoglobin oxygen saturation. This will require 120 litres/hour or about 720 litres for the flight. This information should be available from the airline”.

In addition to low oxygen issues described above, the sites provided further advice for air travel. These advice concerned advanced preparation, and precautions to avoid crises. Advanced preparations included consulting physicians for advice regarding the safety of the travel, obtaining prescriptions for medications that might be needed, organising current medication, obtaining contact information of physician and patients' medical information, and consultation with airlines to arrange accommodation, and inquiring about regulations regarding supplemental oxygen.

In order to prevent crises during air travel, patients were cautioned to avoid aircraft with unpressurised cabins. Where this is inevitable, patients were advised to use precautionary measures such as increased hydration and supplemental oxygen. These measures where advised if patients fly with planes with pressurised cabins that fly at high altitude; "Air travel in pressurized aircraft is safe, but going from low to high altitudes may cause a pain episode. One should drink extra fluids and arrange for supplemental oxygen while on the aeroplane" (US2).

A few messages highlighted the role of psychological stress in causing crisis. Patients were advised to identify their own effective ways of dealing with psychological stresses. The US8 site suggested that patients can reduce psychological stresses by "fostering a positive approach to their condition and to life in general, and being a role model for others".

The sites cautioned patients against the use of use of certain medicines, food and beverages that can trigger illnesses. The medicines included iron preparations, analgesics, and decongestants. Others were recreational drugs including hard drugs, cigarettes, and alcohol. The food and beverages included citrus food, high sodium food, coffee, tea, and dietary soda. More sites cautioned against the use of iron supplementation as anaemia in SCD does not result from iron deficiency. Terms used to describe iron supplements included "iron tablets", "iron medicines" "blood tonics", and "artificial iron". CA1 also cautioned against the use of nasal decongestants such as pseudoephedrine because it can cause crisis by constricting blood vessels. The sites also cautioned against the frequent use of analgesics such as aspirin, 
NSAIDs and paracetamol, but reasons were not indicated. "Fever blockers like aspirin and acetaminophen (Tylenol, Panadol...), Ibupruphen (Motrin, Pediaprophen). Should not be used to lower a fever unless directed by your health care provider" (US2). Few messages advised against the use of cigarette smoking including second-hand smoking, cocaine and alcohol.

Other sites cautioned against the use of use of food, medicines and beverages with diuretic properties as these can cause dehydration and sickling. "Possible harm from too much diuretic effect if excess caffeine or alcohol is taken, because the fluid lost will make dehydration more likely and more tendency for red blood cells to sickle” (US3). Examples of beverages were alcohol, tea, coffee, citrus juices, sodas, diet sodas, and 'doctored' drives. The sites also cautioned against the consumption of high sodium food as SCD is commonly associated impaired kidney function. Sites advised that in situations where intake of this substance occur, patients must increase water intake to replace the fluid loss.

\subsubsection{Interaction of Health Professionals}

A number of messages from the USA, Canada, Uganda, and UK websites recommended regular interaction with health professionals irrespective of whether patients are sick or well. It was advised that when people are well, visits will enable health professionals to examine organs that are more susceptible to complications such as the eyes and dental structures as well as their general wellbeing: patients and parents can discuss their concerns with their health professional, and solicit for ways to enhance their coping skills. Messages on adherence urged patients to comply with medication schedules and dosage and keep to clinical appoints.

Women with SCD were advised to relate to their health professionals regarding reproductive health decisions. Advice on pregnancy among SCD females centred on proactive planning and use of contraception. Because pregnancy among patients is considered a highrisk. Three sites (CA1, US3, US7) advised female patients especially adolescents to plan their pregnancies with their doctors. The sites further advised patients on contraceptive counselling and utilisation. Injectable family planning methods were recommended for its ability to reduce sickling and complications. 
Sites frequently advised patients and families to endeavor to acquire knowledge of SCD whilst they interact with their health professionals. It was recommended that patients, parents and child's family should be well informed about SCD, especially factors that can precipitate crisis and warning signs of crisis:

It may be useful for you to know that there are certain factors that may produce a crisis. These factors include having an infection, such as a cold or the flu, cold weather, being overly tired or fatigue, exercising too hard, being dehydrated (not drinking enough water and other fluids) and not having enough oxygen in the blood. (US7)

It was further advised that new doctors must be informed of the child's SCD status, and patients should endeavour to keep copies of their physiological investigations:

Individuals with sickle cell disease should always request a copy of their complete blood count, reticulocyte count, haemoglobin electrophoresis and special testing or $x$-rays from lab work since individuals with sickle cell disease are at high risk for developing retinopathy (eye disease); they should also request information from tests done by their ophthalmologist recently done. (US3)

\subsubsection{Advice for the SCD Child in School}

A number of sites outlined preventive care actions focusing on SCD children at school. There were two categories of such advice. The first category concerned the role of teachers towards a child with SCD whilst the second focused on parental role regarding their children with SCD. The US2 site advised teachers to acquire knowledge about SCD and its related challenges. Messages on preventive health to teachers were almost similar to those outlined in (Table 12). They included advice on avoiding dehydration, exhaustive activities and extremes of temperature but encouraging adequate hydration. All teacher related messages were retrieved from three sites (US2, CA2, UK). The sites advised teachers to assist parents in developing guidelines for pain management at home and reminded parents about regular medical care follow up care for the SCD child. This US2 site also encouraged teachers to educate classmates of SCD children especially those with severe complications about the 
nature of SCD and solicit their cooperation for the child. It was recommended that this must be done with the consent of the child's parents:

If a child has severe sickle cell disease complications and if the family is willing, perhaps educate classmates about sickle cell disease manifestations such as jaundice, frequent medical absences, decreased endurance, and the need for peers to help the child cope with complicated sickle cell disease. On the other hand, some children have so few sickle cell problems that no special education is needed. (US2)

Another US site advised teachers to observe for academic impairment in the child with SCD and address these. In situations where the SCD child lags behind their peers in academic work due to prolong absence from school due ill-health, teachers were advised to provide extra teaching support in the form of makeup assignment or home-schooling. Two sites (US2, UK) encouraged teachers to create enabling environments where the child will have the opportunity to drink more, rest, urinate frequently, have access to first aid and live a near normal life as much as possible.

Two sites (UK, US3) advised parents to promote awareness of SCD in their children's school by distribution Information Education and Communication materials, or asking SCD specialist to educate the child's school mates. The UK site further advised parents to visits their children's school regularly, and discuss the child's needs with teachers and child's play group leaders. The site also advised teachers to assist the school children to select careers compatible with SCD, and courses that will enable the children to pursue these careers, "Encourage the child to consider appropriate careers at an early age so that he or she can give particular attention to keeping up with the subjects which will be most relevant" (UK).

\subsubsection{Self-monitoring}

The sites advised patients to regularly monitor certain physical and physiological indicators to facilitate early identification of problems. Four areas of self-monitoring involving 17 references were located from the sites including splenic palpation, body inspection, pain trigger monitoring and temperature check. These advice were located in USA, Uganda and Canadian websites. As indicated in Table 13, splenic palpation received the 
highest number of references ( 6 of 17 references) but more websites $(n=4)$ made references to body inspection than the remaining strategies.

TABLE 13. REFERENCES TO SELF-MONITORING OF DISEASE BY WEBSITES

\begin{tabular}{|l|c|c|}
\hline Variable n=17 & Frequency & $\begin{array}{c}\text { Websites } \\
(\mathbf{n = 1 4})\end{array}$ \\
\hline Splenic palpation & $6(35.3 \%)$ & 3 \\
Body inspection & $5(29.4 \%)$ & 4 \\
Pain assessment & $4(23.5 \%)$ & 3 \\
Use of thermometer/temperature & $2(11.8 \%)$ & 1 \\
\hline
\end{tabular}

\subsubsection{Splenic Palpation}

Three sites (US1, UG, CA1) advised parents to learn splenic palpation to facilitate early recognition of splenic enlargement. Different advice was offered for patients with existing enlargement and those without. Parents of children with existing enlargement were advised to conduct regular palpation and additional palpation anytime patient is ill. Patients of children without enlargement were advised on daily palpation, preferably when bathing the child. Parents were advised to seek emergency medical attention when an enlarged spleen is detected or when an existing enlarged spleen grows bigger.

\subsubsection{Body inspection, pain assessment and temperature checks}

The sites indicated the need for regular skin inspection for SCD patients. Almost all recommendations on body inspection (references, 29\%) targeted parents and caregivers. These included observing their children for paleness, jaundice, skin condition, and symptoms of painful crises. Inspection for paleness was the most frequent. Parents were advised to frequently observe specific parts of the body such as the inner eyelids, tongue, palms and lips for "pallor", "pale" and "white", "Watch out for the colour of children's palms, lips and tongue daily if they change from dark to whitelyellow or pale" (UG). Parents were urged to note the usual skin colour of their SCD child to enable them recognise changes. Two sites (UK, US7) indicated that symptoms such as the thirst are predictive signs of crisis and advised patients to note.

Few sites advised on strategies to monitor pain. Almost all these advice concerned identifying pain triggers. The sites advised patients to keep daily journals and record activities 
of days preceding the days they experience crisis. In addition, one site (US2) advised parents to regularly check on their children for fever with a thermometer.

\subsubsection{Self-Diagnosis}

Few sites provided advice on physical and physiological indicators that require urgent attention. Patients were advised to recognise these indicators and seek immediate medical help (Table 14). All self-diagnosis advice were retrieved from US websites except Fever that was located in websites from USA, Canada and Uganda.

TABLE 14. REFERENCES TO SELF-DIAGNOSIS BY WEBSITE

\begin{tabular}{|l|r|c|}
\hline Indicator & $\begin{array}{r}\text { Frequency (\%) } \\
\mathbf{n = 2 7}\end{array}$ & $\begin{array}{c}\text { Websites } \\
\text { (n=14) }\end{array}$ \\
\hline Fever & $8(29.6)$ & 3 \\
Difficult breathing & $3(11.1)$ & 2 \\
Chest pains & $2(7.4)$ & 1 \\
Abdominal pains & $2(7.4)$ & 1 \\
Severe headache & $2(7.4)$ & 1 \\
Abdominal swelling & $2(7.4)$ & 1 \\
Numbness/Weakness & $1(3.7)$ & 1 \\
Difficulty talking & $1(3.7)$ & 1 \\
A typical pain & $1(3.7)$ & 1 \\
Extreme tiredness & $1(3.7)$ & 1 \\
Sudden vision lost & $1(3.7)$ & 1 \\
Unusual headache & $1(3.7)$ & 1 \\
Body weakness/loss of feeling & $1(3.7)$ & 1 \\
Unresolved pain & $1(3.7)$ & 1 \\
\hline
\end{tabular}

Fever was the commonest indicator and described in specific and non-specific terms. Two sites (US2, CA1) reported the level of temperature that warrants prompt action in both degrees Centigrade $\left(38^{\circ} \mathrm{C}, 38.4^{\circ} \mathrm{C}, 38.5^{\circ} \mathrm{C}\right)$ and Fahrenheit $\left(101^{\circ} \mathrm{F}, 101.3^{\circ} \mathrm{F}\right)$. Most sites used nonspecific terms such as "fever" and "high fever": In the event of fever, almost all sites advised patients to seek "prompt" medical help; US2 specified that parents should seek help within one hour. The Uganda recommended the use of paracetamol before sending the patient to the hospital. 
Limited information was located in the sites regarding the remaining self-diagnosis recommendations. These were captured in two quotations from the sites. "The individual should seek medical attention if the following occurs: fever, headache, chest pain, abdominal pain, numbness or weakness" (US6).

"Patients and families should watch for the following conditions that need an urgent medical evaluation: Chest pain, Abdominal swelling, Unusual headache, Shortness of Breath, Pain that will not go away with home treatment, Abdominal swelling, and Sudden vision change” (US2). The US6 site advised parents to observe and report snoring and sleep apnoea among children.

\subsubsection{Self-treatment}

Wide varieties of therapeutic actions were recommended for self-treatment when SCD illness or problems have been recognised. Two thematic subcategories of these actions were observed - managing painful crisis and managing specific conditions. Painful crisis management strategies, involving pharmacological and non-pharmacological measures were most recommended. Of all pain management strategies, pain medication was the commonest (29.9\%). All pharmacological pain management strategies were located from Canada, USA, UK and Ugandan websites. Use of relaxation techniques was the commonest nonpharmacological strategy (11.4\%) (Table 15). All non-pharmacological strategies were located at websites from USA, UK and Uganda.

Few recommendations were located for managing certain specific complications. Legs ulcers management was the most recommended (6 of 12 references) and were located in USA, Canada and Uganda websites. All the remaining recommendations were located in US websites except priapism management which was located in Canadian website.

\subsubsection{Pain management}

Regarding pain management six websites recommended patients take analgesics and seek hospital care if analgesics failed to alleviate their pain. The named analgesics included “acetaminophen”, “paracetamol”, “Tylenol”, “NSAIDs”, “ibuprofen”, “diclofenac”, "voltaren" and "Brufen". Two sites (US2, UK) recommended the dosage of analgesics for home management: 
TABLE 15. REFERENCES TO SELF-TREATMENT

\begin{tabular}{|l|r|c|}
\hline Variable & $\begin{array}{c}\text { Frequency } \\
\mathbf{n = 1 3 1}\end{array}$ & $\begin{array}{c}\text { Websites } \\
\mathbf{n = 1 4}\end{array}$ \\
\hline Pain management (n=32, 24.4\%) & $13(29.9 \%)$ & 6 \\
Use of pain medications & $5(11.4 \%)$ & 3 \\
Relaxation techniques & $4(9.1 \%)$ & 4 \\
Application of warmth & $4(9.1 \%)$ & 2 \\
Increased hydration & $2(4.5 \%)$ & 2 \\
Use of massage & $2(4.5 \%)$ & 2 \\
Use of distraction & $1(2.3 \%)$ & 1 \\
Injury care & $1(2.3 \%)$ & 1 \\
Bandaging & & \\
\hline Mgt of specific complications (n=12,9.2\%) & $6(13.6 \%)$ & 3 \\
Leg ulcers & $1(2.3 \%)$ & 1 \\
Ductility's & $1(2.3 \%)$ & 1 \\
Priapism & $1(2.3 \%)$ & 1 \\
Avascular necrosis of head of femur & $1(2.3 \%)$ & 1 \\
Bedwetting & $1(2.3 \%)$ & 1 \\
Jaundice & $1(2.3 \%)$ & 1 \\
Pain with menses & \\
\hline
\end{tabular}

Pain can also be relieved by pain-killing medicines [analgesics], which can be used at home. The gentlest analgesic to try is paracetamol, given three times a day (62.5 mg under 12 months, $125 \mathrm{mg} 1$-4 years, $250 \mathrm{mg}$ 4-10 years, $500 \mathrm{mg} 10-14$ years, and $1 \mathrm{gm} 15$ years upwards). The next gentlest is codeine phosphate, given four times a day, at 1-2 mg for every kilogram of body weight of the sufferer. A stronger analgesic is a non-steroidal anti-inflammatory agent, such as Diclofenac, which is given three times a day, at a dose of $1 \mathrm{mg}$ for every kilogram of body weight of the sufferer. (UK)

In the event of painful crisis triggered by menses, patients were advised to take ibuprofen 6 hourly during the period of the menses.

The recommended non-pharmacological pain management strategies were rest breaks, deep breathing exercise, and distractions. The others involved the application of heat to the affected area with hot water bottles, warm cloth, topical creams, massaging, and warm baths. It was also suggested that patients should increase their fluid intake as soon as crisis commences. US2 site suggested that increased hydration and pain medications alone were 
sufficient in managing minor painful crises. Two sites (UK, UG) recommended bandaging the affected site.

\subsubsection{Management of specific conditions}

Leg ulcers management recommendations included prevention, first aid treatment for new ulcers and day-to-day care of existing ulcers. Prevention included wearing "compression garments", "proper socks and shoes" and "Taking special care of healing scars (cover them in bandages and socks)" (CA1). First aid treatment advice involve immediate attention to all cuts, scraps and bites, washing with antiseptics, and seeking medical help if patient do not respond to home treatment. Recommended care for day-to-day care of existing ulcers include strict bed rest and elevating ulcerated legs.

One site (US2) advised on prevention of avascular necrosis of the head of femur. Patients were advised to undertake low impact activities such as sitting down and lifting the legs, avoiding lifting heavy objects, and avoid exercises that involve jumping and jogging. Vitamin D and calcium supplementation were recommended but these must be supervised by health professionals to avoid associated complications.

With respect to managing bed wetting, parents were discouraged from using medicines as these are unhelpful. Recommended strategies were reducing fluid intake at night, and bladder training involving asking the child to urinate before bed, using alarm systems to wake the child to urinate, and rewarding the child for success. The same site also recommended home management for jaundice:

If you see his eyes getting more dark yellow than usual, the first thing to try is to give more water to drink and flush the bilirubin out the kidneys by urinating more. That extra fluid passing through his body should make the eyes a lighter yellow colour If the yellow does not get back to usual with more water, then probably something is making the red blood cells break down faster than normal. This could be caused by infection or sickle red blood cells getting trapped in lungs or other parts of the body, and he may need to get to a doctor. (US2) 


\subsection{Self-Management Determinants and Strategies}

A total of 54 references were cited for self-management determinants (contextual, vulnerability, self-management resources). The majority 35 (64.8\%) were concerned with the self-management resource (Table 16). Regarding contextual factors, equal proportions (50\%) were recorded each for school teacher management and avoidance of over protection. References to school teacher management were located from one website each from the USA and the UK. Four websites from the USA, Ugandan and UK sites had recommendations for avoidance of over protection. All the recommendations for social support were located in at least one website from all five countries except Canada. Sites from same countries had recommendations for psychosocial coping.

With respect to self-management resources, slightly more than half $(51 \%)$ of the messages were concerned with acquiring knowledge of SCD. The eight sites that had materials with self-management recommendations were distributed in the USA (6), UK (1) and Canada (1). Four websites from the USA and one each from all the remaining countries had recommendations for social support. Apart from the sickle SCA of Canada, one association from each of the remaining four countries provided the recommendations for coping behaviour. More than half $(55.6 \%)$ of the messages on vulnerability related to vocational and employment selection. Recommendations were located at the sites from the USA (6), UK and Uganda.

As mentioned in the introduction, some self-management determinates (school teacher management, general knowledge of SCD, pregnancy precaution were located within the context of the self-management actions (physiological self-management actions) and have been described within their context. In this section, I describe the remaining determinates which were related to psychosocial concerns and strategies recommended. 
TABLE 16. REFERENCES TO SELF-MANAGEMENT DETERMINANTS BY WEBSITE

\begin{tabular}{|l|r|c|}
\hline Variables & $\begin{array}{c}\text { No.=44 } \\
(\mathbf{\%})\end{array}$ & $\begin{array}{c}\text { Websites } \\
\mathbf{n = 1 4}\end{array}$ \\
\hline Contextual factors (n=10, 18.5\%) & & \\
School/teacher management & $5(50.0)$ & 2 \\
Avoidance of overprotection & $5(50.0)$ & 4 \\
Self-management resources (n=35, 64.8\%) & & \\
Social support and network & $18(51.4)$ & 9 \\
General knowledge of SCD & $11(31.4)$ & 7 \\
Psychological coping behaviour & $6(17.1)$ & 5 \\
Vulnerability factors (n=9, 16.7\%) & & \\
Vocational/ employment selection & $5(55.6)$ & 4 \\
Pregnancy precautions & $2(22.2)$ & 2 \\
Adolescence management & $2(22.2)$ & 2 \\
\hline
\end{tabular}

\subsubsection{Managing Psychosocial Challenges}

As state in the introduction, some self-management determinants were offered within the context of psychosocial challenges and strategies to overcome them. These included over protection, social support, coping behaviour, work and career selection, and adolescent vulnerability.

\subsubsection{Avoidance of Over Protection}

Four sites US $(2,6)$, UK and UG indicated overprotection as a major contextual factor that can adversely sites advised parents to avoid overprotecting SCD children. The messages focused on the reasons for avoiding overprotection and strategies for avoidance. The site indicated that overprotection can "spoilt" the child, and can present difficulty for independent life in adolescence. In order to avoid overprotection, parents were advised to avoid giving special attention to SCD children, and allow them to live independent life after 18years.

\subsubsection{Social Support and Coping Behaviour}

The sites also recommended a range of self-management resources that patients can be accessed to or cultivated to enhance their self-care. These included social support and coping 
behaviour. Social support was the commonest resources that sites advices patients to access to deal with psychosocial challenges. The messages centred on people who can provide the support and types of support required by SCD patients. The recommended people who can provide support include: family, friends, and support groups such as sickle cell associations and social institutions that provide support services. The type of support required concerned academic issues, career selection and life planning, transportation, domestic chores, love and care: "Family love and support can help ease the painful episode and keep the patient looking ahead to a career and a family of their own." (US2). The importance of joining support groups such as the sickle cell association was highlighted by many websites. These benefits include assistance and advice to patients, sharing of experiences, frustration, challenges, hopes and success with their peers.

A few sites shared advice on psychological coping strategies for SCD related problems. The strategies include: developing a positive attitude towards problems, improving the social status of the person with SCD, and seeking advice from health professionals. The sites encourage patients to develop positive attitude when confronted with SCD challenges. Patients were advised to avoid denial and worrying, and confront problems with a focus on what will promote normal functioning. The Ugandan site advised parents to aim at improving the social status of their SCD child in order to minimise stigmatisation; "They should be groomed into responsible citizens to avoid stigma and discrimination” (UG). The site recommended that SCD patient should consult their health professionals during their stable conditions to learn ways of coping and how complications could be minimised. The consultation should also provide them opportunities to discuss their concerns with the health professionals.

\subsubsection{Work, Career Issues and Adolescence Vulnerability}

As SCD patients can face work related challenges due their vulnerability two sites (US3, UK) advised that teenagers and children with SCD must be assisted to select careers that are consistent with the condition. Teenagers were advised to consult relevant community agencies for assistance:

At this stage of life, teenagers should give serious consideration to college, careers, and their future as adults. They should be encouraged to consider careers that are 
consistent with their medical condition. Referral to key community resource agencies such as departments of employment, social or youth services can offer important linkages. (US3)

The UK site further advised patients to declare their sickling status to their employers and acquaint themselves with work related policies such as benefits, access to work, and disability discrimination policies.

Vulnerability among adolescents was highlighted by US3 and US5. The advice targeted adolescents experiencing physical, emotional and social challenges due to SCD complications. As adolescence may face challenges, decisions making about education, employment and independent adult life, it was recommended that the support agents should organise transition programme to prepare adolescent. To manage these challenges, the sites recommended that the content of these programmes should include a support group counselling systems, sexuality and contraception advice. The Association (US5) organised programmes that taught social, education, employment, financial literacy, health and wellness, self and life skills.

\subsection{Summary}

The SCD focused websites reviewed provides a wide range of recommendations for physical and psychosocial self-care. The advice were: medicinal and non-medicinal actions people must endeavour to use to promote their health, medicines and non-medicines people must avoid or do in moderation to avoid exacerbation of disease, physical and physiological monitoring people must undertake on regular basis, emergency signs people must recognise and take immediate actions, resources that must be accessed or cultivated to support physical and physiological care. The sites advised that interaction and collaboration with health professional and teacher for SCD child at school are important. 


\section{Chapter 7: Perspectives of Health Professionals}

This chapter presents the results of the interviews with health professionals in Ghana. The results have been organised under two thematic areas - physical self-care and psychosocial self-care. Physical self-care recommendations capture self-monitoring, selfdiagnosis, self-treatment, and consultation and interaction with health professionals. Psychosocial self-care concerns strategies health professionals recommend for dealing with non-health challenges confronting SCD patients and families. The chapter is organised into three sections. The first section describes the characteristics of the professionals. The second section focuses on the physical self-care recommendations, and the third section on managing psychosocial self-care. Quotations are used to support the findings; quotations from nurses are by number, for example NUR1 and for the physician and physician assistant as DR1. The term patients is used to refer to adults with SCD and unless specified also in reference to children and the parents of children with SCD.

\subsection{Study Population}

The study population comprised nine health professionals with varying years of experience with SCD care (nurses [n=6], doctors $[n=2]$, physician assistant $[n=1]$ ). The years of experience providing SCD care ranged from 4-19 years (average 8.6 years). Four nurses were part of the NSSCD Project; their role was to deliver patient education and counselling for parents of children with SCD and adult members of the SCAG. The other two nurses and the physician assistant were coordinators for the SCD clinics in their respective district hospitals. The two physicians were paediatricians and have been coordinators for the SCD clinics in their respective hospitals. One physician had worked on the NSSCDP for more than 10 years. Only one professionals, a nurse had received formal SCD training in the USA. The remaining all learnt on the job. The majority had received five days in-service training in 2011 by the Sickle Cell Foundation of Ghana (Table 17).

All nine health professionals were involved in discussing self-management to patients but the extent of involvement varied. Although the focus of this study was not to link professional's background to their relative contribution to the responses, a brief highlight is presented. The professionals with experience with the NSSCD Project shared more comprehensive information regarding self-management than those who were not part of the 
project. Generally, the nurses provided more information on what patients could do at home whereas the physicians tended to recommend clinical based treatment. The number of years of experience working in SCD care influenced the comprehensiveness of the professionals' selfmanagement knowledge; Professionals with more experience provided more detailed responses than those with less experience.

TABLE 17. CHARACTERISTICS OF THE HEALTH PROFESSIONAL PARTICIPANTS

\begin{tabular}{|c|c|c|c|}
\hline Name & $\begin{array}{l}\text { Years of experience in } \\
\text { SCD }\end{array}$ & $\begin{array}{l}\text { Formal } \\
\text { training in } \\
\text { SCD }\end{array}$ & Role with SCD \\
\hline NUR1 & 10 years + & $\begin{array}{l}\text { Multiple } \\
\text { workshops }\end{array}$ & $\begin{array}{l}\text { Education and counselling of patients } \\
\text { and parents. Involved in Newborn } \\
\text { Screening for SCD Project (NSSCDP) }\end{array}$ \\
\hline NUR2 & 4 years & $\begin{array}{l}\text { Workshop (5 } \\
\text { days) }\end{array}$ & $\begin{array}{l}\text { Nurse in-charge of SCD clinic (District } \\
\text { Hospital) }\end{array}$ \\
\hline NUR3 & 19 years & $\begin{array}{l}\text { Formal training } \\
\text { in USA }\end{array}$ & $\begin{array}{l}\text { Education and counselling of patients } \\
\text { and parents. SCD researcher. Member } \\
\text { of Board of Directors for Sickle Cell } \\
\text { Foundation. Involved in NSSCDP. }\end{array}$ \\
\hline NUR4 & 8 years & $\begin{array}{l}\text { Some } \\
\text { workshops }\end{array}$ & $\begin{array}{l}\text { Education and counselling at SCD } \\
\text { clinic/Association. Involved in } \\
\text { NSSCDP. }\end{array}$ \\
\hline NUR5 & 7 years & $\begin{array}{l}\text { Some } \\
\text { workshops }\end{array}$ & $\begin{array}{l}\text { Education and counselling at SCD } \\
\text { clinic/Association. Involved in } \\
\text { NSSCDPOInvolved in NSSCDP. }\end{array}$ \\
\hline NUR6 & 5 years & $\begin{array}{l}\text { Some } \\
\text { workshops }\end{array}$ & $\begin{array}{l}\text { Parental education and counselling for } \\
\text { newly diagnosed SCD babies }\end{array}$ \\
\hline$\overline{\mathrm{DR} 1}$ & 10 years+ & $\begin{array}{l}\text { Multiple } \\
\text { workshops }\end{array}$ & $\begin{array}{l}\text { Physician in-charge of SCD clinic } \\
\text { (District Hospital). Involved in } \\
\text { NSSCDP. }\end{array}$ \\
\hline DR2 & 10 years + & $\begin{array}{l}\text { Workshop (5 } \\
\text { days) }\end{array}$ & $\begin{array}{l}\text { Former physician in-charge of SCD } \\
\text { clinic (District Hospital }\end{array}$ \\
\hline DR3 & 4 years & $\begin{array}{l}\text { Workshop }(5 \\
\text { days) }\end{array}$ & $\begin{array}{l}\text { In-charge of SCD clinic (District } \\
\text { Hospital) }\end{array}$ \\
\hline
\end{tabular}

Note. $\mathrm{DR}=$ Doctor or Physician Assistant / NUR =Nurse 


\subsection{Physical Self-Care Recommendations}

This section presents the professional's recommendations for physical self-care under the headings: preventive health, self-monitoring, self-diagnosis, self-treatment, and consultation and interaction with health professionals.

\subsubsection{Preventive health}

Preventive health advice covered health maintenance advice and preventive care. Health maintenance advice was in reference to actions patients were encouraged to perform on a daily basis. Two areas of such advice were given. The first consisted of actions patients could perform independently without consulting health professionals including: maintaining adequate hydration to prevent dehydration, maintaining adequate nutrition, maintaining personal hygiene and using supportive medication. The most common of these recommendations related to diet and hydration. The second related to health professional directed actions patients were required to perform. Key actions were adhering to prescribed medications, attending routine health checks, and seeking clinical care for problems and complications.

All nine participants advised on eating a healthy diet to maintain health. The first set of these advice related to the type of diet for SCD patients mode of eating. It was recommended that SCD patients eat a normal diet, which must be "good", with a balanced selection of food. For children, two professionals recommended breastfeeding, normal diet and food supplements for those over six months. The concept of good diet was captured by expressions such as "nutritious," "well balanced," "nourishing," and "eat very well." Three professionals (DR1, DR3, NUR2) indicated that a good diet contains fish, eggs, green leaves, fruit, roughage, lots of meat, and patients should not take meals without stew (in Ghana stew is a sauce). NUR6 commented that patients must acquire the skills to enable them to provide the right amount, combinations, and calories that are required of good diet. Two professionals (DR1, NUR6) referred to the high cost of food and the low economic status of most SCD patients and parents that make it difficult for patients to acquire high quality food such as milk. Consequently, DR1 advised on a selection of cheaper local alternatives: 
You know milk is a luxury in Ghana... Other forms of protein like groundnuts, "agushie" and "akatoa" and the rest... are available in their farms, they should eat them rather than selling them to the rich patients in the cities. Apart from breast milk you will meet some patients who have not taken... a can of milk before, they've not tasted it before, and so it's a luxury in Ghana. (DR1)

Professionals also recommended SCD patients eat regularly to promote good health, "we advise on small chunks of food more frequently rather than three main meals" (NUR3).

The second set of advice focused on food promotion. The messages on good diet were frequently linked with an explanation of the importance of diet to the health of the SCD patient. A good diet was said to help prevent and restore anaemia and under nutrition, and improve patients' immune system. Such messages were intended to motivate patients to cultivate the habit of healthy eating. As part of this motivational strategy, two nurses (NUR1, NUR5) explained the physiological relationship between a good diet and under nutrition, "And we also advise them to take well-nourished diet. They need good nutrition to be able to develop well, because their red blood cells are being destroyed at a faster rate almost every 21days" (NUR1). An aspect of messages on food promotion addressed misconceptions about food, mostly in relation to fats and oil consumption. The professionals referred to these when they recounted their experiences regarding patients associating oils as the cause of their jaundice, and the belief by patients that oil is unsafe for their health. Consequently, the advice to correct food misconceptions usually focused on explaining the health benefits of oil to the body, and its role in enhancing the aesthetic value and taste of food:

They come in with funny, funny...ideas; they say I shouldn't take in a lot of oil, if you watch, my eyes are always yellow. They come in with that thing at the back of their mind, but we tell them too much of anything is not good. There is no need to have too much oil in your food...it's not good, there is no need to add too much sugar in your "koko" or whatever, too much of everything is bad...but what they have in mind is oil is not good for them, you tell them when you don't use oil in your food it won't taste nice. Yes use the oil alright but not too much... But we never advise them to stop... and we ask them to take in a lot of meat; protein. (DR1) 
A food misconceptions raised by NUR4 was related to the consumption of protein foods: "They have a lot of misconceptions such as not to eat egg, groundnuts (peanuts) so we let them know that they are good for them".

The second commonest health maintenance advice concerned the need for patients to drink oral fluids frequently to hydrate their body. This advice was referred to by all except DR3. The advice included the rationale for hydration, types of liquids, and mode of drinking. The advice promoted hydration by encouraging patients to cultivate the habit of liberal fluid intake irrespective of feeling of thirst. Such messages tended to include an explanation of the link between habitual fluid intake, dehydration and crisis.

And then realising they haven't taken in enough water, and then their UTI...their urine become dark. Dehydration too is one of the precipitating factors for their crisis, so anytime they get dehydrated...it can precipitate their crisis so we advise them...liberal fluid intake and then report to us when necessary. (DR1)

Regarding types of fluids, no particular liquids were specified but water was referred to frequently. Three professionals (DR1, NUR3, NUR4) expressed concerns about the difficulty they faced in getting patients to take sufficient water to meet their daily fluid requirements. These professionals emphasised the need for patients to use a variety of fluids including fruit juices and soft drinks in addition to water to make it easier for children in particular to drink more: "liberal fluid intake, no child will like to take always water, water, water...at times orange juice, pineapple juice, ahaa, it all help them, they like them. A lot of juice, a lot of drink, a lot of fluid and then" (DR1). On how patients should drink, professionals recommended that fluids be taken in small volumes at frequent intervals. In addition, there must be easy access to water for children at home and school.

Regarding personal hygiene, three professionals (DR4, NUR3, NUR4) specified that they advise patients to pay attention to their hygiene to promote wellbeing and prevent crisis occurrence: "We advise them that at home what they should observe is their personal hygiene. You know when they observe personal hygiene, it will help them. So that it will not trigger them" (DR4). 
Six professionals (DR1, DR3, NUR2, NUR3, NUR5, NUR6) advise patients on regular (daily) intake of certain supportive medications including folic acid, B Complex, multivite and penicillin prophylaxis. NUR6 indicated how patients should acquire these medicines.

When their condition is stable we give them their routine folic acid and penicillin $V$... we educate them to take it every day. Even when they cannot come to the clinic they should go and buy some and continue taking it.

Folic acid supplementation was recommended the most. Professionals tended to comment on the benefits of folic acid to SCD patients in relation to blood production. In addition, the routine use of penicillin prophylaxis as a means to preventing infectious diseases among SCD children was advised by some professionals:

In the area of health maintenance of course the new-borns we discuss about the need for parents to put the children on penicillin prophylaxis to reduce infection especially pneumococcal infection. And also we refer them to the sickle cell clinic where they are put on the basic medication such as the penicillin and then also folic acid. (NUR3)

Almost all professionals considered visits and interactions with health professionals as important for patient's self-care. As part of this advice, the professionals highlighted the importance of patient's adherence to prescribed medications and regular follow-up visits. Such advice was mostly targeted at parents. Professionals often highlighted these recommendations whilst complaining about challenges they encountered in getting patients to adhere to their prescriptions medicines and clinical follow-up in the absence of a crisis:

You see some of these children, they won't take their medicines unless they are in crisis or when they start to experience those signs and symptoms and for them we always advise them that they should always take their folic acid daily and anytime they come to the hospital the medication we give them or what they have in the house they should take them. (DR3)

In addition to routine follow-up, the professionals expressed the need for patients to attend clinic for acute care. Four main areas were noted for when patients should seek hospital 
treatment for acute problems. The first area addressed situations when patients have substantive problems or sickness such as a painful crisis or dactylitis:

So if we take the vaso-occlusive events it can happen in the form of painful crisis where the patient complains of pains in the joints and...it is very obvious because the person will be screaming and when that happens depending on the level of pain, we advise them to the hospital for hospital management. (NUR3)

The second area was with respect to situations when patients anticipate an impending problem or observed a warning sign based on their assessment of their condition:

We also educate them about some warning signs and the fact that immediately they see them they are supposed to report to the clinic. Warning signs like jaundice when they appear, abdominal pains, vomiting, diarrhoea...I think the earlier they report to the hospital the better because we have to start treatment immediately. So we make them aware of the warning signs and we encourage them to come quickly to the clinic immediately they see those warning signs. (NUR2)

The third area is the situations where patients have tried home remedies which were unsuccessful or they have problems that they are unable to cope with:

And therefore we teach them that as soon as you see that your child has a fever, reduce the fever by sponging, give the child some antipyretic like paracetamol and then bring the child immediately to the hospital so that they can identify the cause of the fever. (NUR3)

Professionals also advised patients and parents to take advantage of the availability of clinics to receive care at the facilities and advice for home care. Such advice was often associated with explanation of the benefits of attending the hospitals including opportunity to share ideas with patients with similar conditions; find role models in patients who have lived with the condition to advanced ages; and learn from the experiences of others. Professionals considered that such opportunities would increase patients' surviving the disease and improve their psychological well-being. NUR6 shared: 
We have the SCD clinic which is also very influential in the care of these clients because you happen to meet patients who are also going through the same situations and most of them have come out. We have doctors, we have nurses so if they get the chance to speak with these patients it boosts their moral and especially it impacts them to have in mind that they can also lead a better life even though they have the disease. So I would advise all these clients to be part of the clinic, the sickle cell group.

NUR1 advised patients to collaborate with other medical specialty areas such as orthopaedics, obstetrics, nutrition, physiotherapy and nutrition to enhance the management of their children with SCD especially where there are complications:

For a child with SCD as much as you would want to see a paediatrician who is trained in sickle cell at a certain point in time certain conditions may go beyond the paediatrician. For example, if you have a condition that affects the bones because SCD patients...sometimes develop hip dislocation and at that point they need orthopaedics, surgeon will have to come in or if there is any problem with the spleen that will need surgery or any other condition that will need surgery even with the child or sickle cell client, a surgeon to come in to support with the treatment of that child. If they are females too and they grow up. Obstetricians and gynaecologist will also come in to address their health care needs. So, it's a kind of a whole sector of health care workers and comprehensive kind of care will be needed. (NUR1)

In spite of these benefits, the professionals acknowledged some barriers, especially financial barriers, limiting patients' access to health services. All but one professional (NUR1) argued that poor financial status served as barrier to access and affordability to care; it limits patients' ability to access routine follow up, resulting in default. Patients are also not able to purchase medication, health insurance and other resources to manage their condition. NUR2 recounted her experience in relation to this:

And then secondly is finance. That is very difficult for us because sometimes we even come in to help, so taking care of them is very difficult...they don't even have national insurance so we advocate and we could get some sponsorship from the 
insurance office so that it will be made free of charge for them and then it will be done for them and then when they have the insurance they are sure they can buy their drugs but even with that when they come we have to give them some money for transportation to go back home. And some of them have defaulted we call them, some them they give their numbers and we call them and it's not even going through so public health department when they go we give them addresses to follow up but some of them say they don't have money to come for the clinic.

Other barriers professionals commented on concerned insufficient knowledge and technological know-how to manage common SCD complications, lack of effective SCD focused policies, and limited enabling resources. It was noted that a lack of technology and man-power to manage complications such as renal failure and priapism limits the care patients with SCD receive at hospitals. Professionals further acknowledged that even where SCD services were available, issues with community enabling resources including poor telephone networks, poor roads and transportation difficulties limit patients accessing the services. Consequently, patients may not report for follow-up unless they are sick.

To address financial concerns, nurses said they advise patients to register with the National Health Insurance Scheme to help with their health finances. One nurse (NUR4) advised patients to engage in economic activities to raise income, and another (NUR2) gave direct financial support to patients and families. A third nurse noted she advised patients to plan financially for emergency illnesses.

I think they should plan, the family should have a plan. I mean in terms of monitory you know planning into the future, whereby they can plan for emergencies. When there cases of emergency where are they going to get money to care for the child in cases where there is kidney failure or something. Are they going to be able to foot the bill so they should plan towards emergencies, life insurance they should look into that if it is possible? (NUR6)

\subsubsection{Preventive care advice}

Preventive care advice concerned messages that focused on what must be avoided or performed with caution to prevent or avoid triggering problems and complications. The 
majority of these recommendations related to painful crisis. The messages were concerned with avoiding extreme temperature, preventing dehydration and reducing over-activity. Other recommendations related to avoiding food substances that can trigger crisis and personal hygiene.

Eight professionals advise patients to avoid exposure to extremes of temperature. All but one of these recommendations were in relation to exposure to cold temperatures. NUR3 described how cold and hot temperatures could precipitate crisis:

Sickle cell patients should avoid extremes of temperatures. Where they are sitting in very cold environment or too hot environment where they sweat and get their temperatures suddenly reduced because their bodies more or less is reactive between temperatures. (NUR3)

The professionals advised patients to avoid cold temperatures by avoiding conditions or activities that expose an individual to extreme cold. Conditions to avoid included exposure to cold weather without adequate warm clothing, not taking cold baths or cold drinks, and not walking bare chested or in the rain. The commonest advice related to cold baths. Professionals tended to support their advice with physiological mechanism between cold and crisis. "They should avoid cold because with SCD their blood needs to circulate well in order to avoid crisis" (NUR1). Patients were also advised on how to prevent the impact of cold on their health such as appropriate dressing for the weather and having warm clothing such as cardigans, pullovers and jackets with them at all times. Preferably these clothing items should cover the chest and have long sleeves. Professionals further advised patients to take warm baths and wear warm clothes to keep themselves warm after being beaten by rain.

Many professionals highlighted the need for SCD patients to ensure moderation with activities. Seven professionals (DR3, NUR1-6) recommended strategies in the area of safety with activities. Recommendations regarding activities that patients must avoid comprised of activities that involve use of physical strength, are vigorous or strenuous in nature, for example weeding, athletics, football, and carrying a load. Two nurses (NUR1, NUR4) advised that patients engage in moderate exercises but cautioned patients to stop and rest when they reach their energy limit. Most of the advice was accompanied by an explanation of the relationship between over activity, oxygen deprivation and painful crisis: NUR1 shared, 
We ask them to avoid strenuous exercise when they are in school and they want to do some athletics because its strenuous you labour too much, because when the oxygen saturation is too low it will cause problems so too much strenuous work like weeding at school and stuff must be avoided.

Whilst most of the advice was directed at children, NUR5 had advice for adults in "selfemployed not to overwork themselves".

Strategies to mitigate the impact of over activity on the health of SCD patients were noted. These included body-awareness, use of hydration, and exemption from activities. Regarding body-awareness patients were advice patients to be aware of their energy limits during physical activities. At the point of exhaustion, patients are advised to stop, drink lots of fluids and rest. Three professionals advised that school children must be exempted from strenuous activates at school. In line with this, parents were advised to provide medical certificates from their health professionals for the school authorities.

The professionals cautioned patients about particular food items. Nurses consistently advised patients to avoid or reduce the consumption of food and beverages that have the potential for triggering illnesses. The physiological relationship between caffeine and crisis explained by NUR4.

We advise them to avoid food that contain caffeine because it constrict the blood vessels and that can trigger the crisis. So caffeine food we usually advise that they either reduce it to the minimum or they should avoid...So apart from these, some food when they have taken and they become allergic to that food we also tell them that they should avoid some of these food but they don't have a restricted food to take. (NUR4)

Alcohol and food that elicit allergies were advised to be used with caution.

Professionals also advised patients to avoid infectious disease including malaria, pneumonia and worm infestation as potential causes of crises and anaemia. The commonest advice related to malaria. One aspect was use of the Insecticide Treated Bed Net (ITN). "They should be sleeping in ITN because when they get malaria, sometimes it triggers their crisis so 
they should avoid mosquito bites as much as possible" (DR1). A second aspect concerned the use of prophylaxis treatment but no specific medicine was specified.

Some advice was specifically targeted at addressing triggers. Adult patients were advised to avoid harmful lifestyles such as alcoholism, smoking, exposure to overcrowding, dehydration, and overworking (NUR1, NUR3, NUR6). Three professionals (DR2, NUR1, NURX) also advised patients to endeavour to prevent injuries, as wounds to SCD patients are difficult to heal. DR2 targeted her advice for splenic injuries towards boys, "And when they are playing football, the boys they should avoid contact as much as possible... When you playing ball make sure nobody touches your stomach. They should protect themselves". Another professional (NUR3) also cautioned SCD patients to be aware of the risk for conditions such as CVA and complications of the liver.

\subsubsection{Self-Monitoring}

Regarding self-monitoring, only six professionals (DR1, DR2, NURs1-3, NUR6) outlined physical and physiological indicators that they advise patients to check periodically, including: pallor, urine colour, fever, jaundice, splenic enlargement or pain, pain, and general demeanour (for children). The commonest recommendations concerned pallor, urine colour and fever checks.

\subsubsection{Checking for pallor, urine colour and fever}

All six professionals advised on regular checks for pallor. The advice centred on where, how, and why patients should check. The conjunctiva, palms and nail beds were recommended as the places to check. Different strategies were noted for checking pallor among adults and children. NUR1 commented that for children:

They should also be observing the child from time to time for example the conjunctiva and the palm for colour change so that when the baby is getting pale they can compare the baby's palms to theirs they can see the difference in terms of colour so that they can report as soon as possible.

And, NUR2 shared what she advised adults for checking pallor: 
We educate them on how to check the conjunctiva for anaemia that is how they will get to know the warning. They will stand in the mirror and check if your eyes is yellow and you check your conjunctive and its pale like you look in your palms and you are pale you can report to the hospital.

The advice on urine checks focused on two areas: what should be checked, and the frequency of checking. On what should be checked, the two doctors specified the type of changes (co-cala colour or dark urine) patients should look for. Professionals frequently related these changes as indicative of physiological abnormalities such as dehydration, vasoocclusive crises and kidney malfunctions, and urged patients and parents to report immediately to hospital when these signs are observed. In terms of frequency, DR1 advised patients to monitor their urine colour in the morning and evening.

Of the six professionals, four (DR2, NUR2, NUR3, NUR6) advised on strategies for fever checking. The need for fever checks among children was highlighted. Strategies noted include using a thermometer or the back of the checker's hands. It was noted that regular fever checks help patients to recognise complications such as impaired blood supply to parts of the body due to vaso-occlusion. Professionals reported advising patients not to consider all fever as malaria and to avoid taking antimalarial medications for all fevers.

Many participants considered it important for patients to acquire thermometers at home. However, there were mixed opinions about ability to purchase and use thermometers. Whilst five of the six participants thought patients could not afford thermometers, NUR6 thought that thermometers are cheap and within the purchasing power of most Ghanaians. She mentioned one retailer were patients could buy thermometers at an affordable price (less than $1 \mathrm{GH})$.

\subsubsection{Splenic palpation, pain and others minor checks}

Two professionals (NUR3, DR2) advised routine splenic palpation. This advice was targeted at patients with existing enlargement and those without. For "those who have splenomegaly, we tell them that they should take note of where the size is, so when they see that it's becoming bigger or its becoming tender they should report to the hospital" (DR2). Those with no apparent splenic disorder were advised to routinely; 
watch out for the spleen. If the child is a new-born their mothers are taught how to palpate the spleen. If he is a young adult or an adult, again we teach them how to palpate the spleen so that they will be able to determine when their spleen enlarges so they can act appropriately...Then we also teach them how to palpate the spleen of the baby so that they can look out for splenic sequestration which is one of the major complications of SCD. (NUR3)

Three professionals (DR2, NUR1, NUR6) also commented that mothers should monitor their children for prolong or excessive crying as indication of painful episodes. NUR6 advised parents to assess their child for areas of tenderness, if the children cries for a long time to determine whether or not they are in a crisis.

There were other minor recommendations that were mentioned by individual professionals. These included observing for general demeanour, jaundice and monitoring growth, and if there were changes in these report them to the clinic. DR2 advised parents to monitor

their [child's] general growth, because, some of them have delayed growth so especially those that are screened, the new-borns we tell the mothers to be observing their development if by one and half years the child is not walking, they should report to the hospital. Then we will follow her up and see if anything wrong or just the sickle cell.

\subsubsection{Self-Diagnosis}

All the professionals provided some advice on self-diagnosis and used terms such as "emergency signs", "warning signs", and "complications" to describe diagnostic indicators. These indicators included fever, jaundice, unusual feelings, enlarged or tender spleens and prolong crying by children. The advice on self-diagnosis was aligned with self-monitoring. The major indicators highlighted were fever and changes in urine colour.

\subsubsection{Fever and urine colour changes}

There were objective and subjective recommendations to what constitutes the degree of fever that patients should recognise as problematic. Professionals either specified a 
temperature level of $37^{\circ} \mathrm{C}$ or higher (DR3, NUR4) or advised patients to note when the body is warm to touch. The relationship between fever, infection and painful crisis was explained as follows;

And then if it comes to the infections, of course the infections always exhibit by high fever and once the temperature is beyond $37^{\circ}$ [Centigrade] degrees you should know that your child is running temperature. And we make them aware of the seriousness of temperature and the fact that there may be an underlying serious infection such as pneumonia. (NUR3)

Change in urine colour was the second commonest indicator the professionals recommended for self-diagnosis. Three professionals (DR1, DR2, NUR2) specified dark or coca cola urine as an abnormal sign that required attention. DR1 recounted his experience with how patients' self-diagnose and refer themselves regarding urine colour changes:

So the parents...the patients we advise them to look after themselves well, self-refer yourself then we find the problem... One child, interestingly will be walking playing in town and then walk to the hospital, 'doctor, me gyunsっe no me gyunsっ ani aye tuu' [My urine has turned dark]. Then you find some fluids for them to drink then urinate again then you see, apart from that they are well.

\subsubsection{Other minor self-diagnostic indicators}

Four professionals (DR2, DR3, NUR2, NUR5) referred to jaundice as a diagnostic sign. Patients were advised that if they developed emerging or worsening jaundice they should seek immediate care as this could indicate an emerging or worsening haemolytic crisis. Regarding anaemia, two professionals (NUR3, NUR5) advised patients to frequently monitor by evaluating the pallor of the conjunctiva and the palms to identify anaemia. A doctor and the physician assistant also advised patients to consider any unusual signs atypical of their SCD as problematic:

Patients were also advised to note any enlargement or pain at the spleen area which may indicate splenic sequestration. Two professionals (DR2, NUR1) noted that a child who cries profusely must be suspected of experiencing a painful crisis. Other indicators, mentioned 
in passing without further elaboration were dactylitis among children, diarrhoea and vomiting, coughing, priapism, lump in the abdomen, dizziness, fits and collapse, swollen joints, pain in joints, abdomen or chest, and difficulty in breathing.

Whilst advising patients on indicators, seven professionals (DRs1-3, NUR2, NUR3, NUR5, NUR6) raised concerns about patient's lack of knowledge and their inability to recognise problems early. Limited knowledge about the condition and its causes meant that patients can neither anticipate complications nor take appropriate action. Lack of knowledge of SCD and self-care was frequently linked by the health professionals to limited formal education. In the light of this, all professionals recommended that patients acquire general knowledge about SCD in order to understand the problems they must anticipate. Three nurses (NURs1-3) considered that topics patients should know are facts about SCD including common signs and symptoms of complications such as anaemia, painful crisis, infection, and big abdomen.

DR1 reported advising patients to acquire self-monitoring and diagnostic skills including temperature checking, splenic palpation, detecting pallor, and to tell the difference between a sick and well child:

Making sure you are able to take the temperature, "ah my child is hot", and the skill of seeing the difference between your child, when your child is well and when your child is sick, some children when they are sick they go to their room and sleep, when they feel sick, then the mother says "ah!...my child is sick because he usually does this, but today look at him lying down quietly”, then ...some patients bring...their children to hospital like that. Then the skill of detecting pallor, they've been taught to look under tongue, look under oral mucosa, look at the conjunctiva, the palm of their children and whenever they see their children are pale, from the usual...steady state like pallor they should bring the child to hospital, and...the mothers will come, ei doctor, 'mehweE na ni ase no, na ni ase ays fitaa' [when I look at the eyes and they are pale]. We also examine and do the $H B$, you will be surprised some of them having 4.0, 3.5 and they are walking about. Because of their chronic anaemia...they are able to walk about any how with low $H B$. 
In order to improve patients' knowledge, the professionals reported that they partner with other public health providers to promote education on SCD during their outreach programmes.

\subsubsection{Self-Treatment}

Two broad subcategories were derived from the professionals' interviews regarding self-treatment - self-treatment advice and self-treatment observations. Advice concerned recommendations given to patients, whilst observations concerned practices the professionals have observed patients undertaking.

\subsubsection{Self-treatment advice}

Pain and fever management was the focus of most self-treatment advice, and there were a few recommendations concerning dactylitis, anaemia, pneumonia, and priapism management. All professionals except NUR5 commented on advising regarding pain management. The recommended measures were use of analgesics, hydration, heat applications, massage, relaxation and periodic movement. All eight professionals who advised on pain management noted the importance of analgesia. In addition to this nurses also recommended using hydration for pain management. Most advice on analgesics was mentioned without specifying names of particular medicines. Where these were specified, paracetamol, ibuprofen or NSAIDS were recommended: In terms of hydration, patients were mostly advised to drink lots of "water" or "fluids" generally:

When they have crisis in fact because most of them is the pain crisis (VOC) we tell them to drink fluids, lots of fluids, take brufen [ibuprofen] and report immediately to the hospital...They should just take stat dose of brufen and come to the hospital so that we will assess and see how severe it is. (DR2)

With respect to heat application, four nurses (NUR1, NUR3, NUR4, NUR6) advised the application of hot compresses to the painful body part. NUR4 explained the physiological mechanism underlying heat and pain relief in SCD: "it will help them for the blood flow into the area. And then when there is dilatation, it will help for the blood to flow". Among children who cannot verbalise pain, NUR1 recommended. 
Whenever babies are crying so much or unnecessarily, you should know that they may be in pain so they should try and apply hot compresses or warmth around their body, they may not know specifically which parts of the body is paining so they can ... keep the baby warm or put some hot compresses around every part of their body or they can even make some warm water and put the baby in the warm water.

NUR3 noted that she recommended that patients experiencing painful crisis should not confine themselves but should pace up from time to time. And NUR4 advised patients to massage the affected part using mild creams such as "Vaseline".

Four professionals (DR1, NUR3, NUR5, NUR6) recommended patients manage fever at home. Three of the four considered the use of antipyretic medication (e.g., paracetamol) and tepid sponging with lukewarm water. For children experiencing fever, NUR6 recommended giving more fluids. She also described that tepid sponging should be conducted from the feet upwards. However DR1, advised patients to seek immediate care at the clinic when they experience fever without any home interventions.

Three professional (NUR2, NUR3, NUR5) advised parents on dactylitis management among children. The advice included increasing hydration for the child, gently apply soothing cream or topical analgesic to the affected part, and giving pain medication. Parents were cautioned against vigorous rubbing of the affected area since this can result in bleeding. Only one professional (DR3) commented how priapism should be managed at home: "So the male patients we tell them that as soon as they see something like that they should drink the fluids and come to the hospital then we manage" (DR2).

Professionals also referred to skills patients should acquire for self-treatment. Resources included medicines patients must keep at home. Two physicians advised patients to have pain medication such as paracetamol, ibuprofen or other non-steroidal anti-inflammatory drugs (NSAIDS) for first aid management for painful episodes, and DR2 advised patients to have folic acid and penicillin V. Two professionals (NUR2, NUR3) also advised that patients should acquire the skills to manage minor illnesses and injuries at home. 
We teach them how to tepid sponge which is one of the first aid in the house is when the child has fever...administration of the drugs, we teach them and they have that skill. Sometimes we teach them a little about... how to treat the wound in the house. (NUR2)

\subsubsection{Self-treatment observations}

Self-treatment observations reported by the health professionals centred on the management of pain, anaemia, leg ulcers and fever. The strategies observed involved traditional and conventional measures. The commonest of these observations concerned pain and anaemia management

Many professionals shared their experiences regarding pain management practices among patients. The strategies reported involved traditional medicine, mechanical interventions, and conventional measures. Five professionals (DR1, NUR1, NUR2, NUR4, NUR5) had observed the use of herbal medicines and spiritual interventions in the management of painful crises. Only some of the herbs, numum, alcobalm, were specifically known by professionals, others were not. NUR2 shared that patients tended to use herbal medicines when they did not experience relief from their symptoms from conventional medicines: "Some of them they talk about herbal medicine especially when they find that they have been taking folic acid and everything and they still get the crisis. Sometimes they want to go herbal instead' (NUR2). It was reported that patients used the herbal medicines in different ways. They are taken orally as concoctions, applied topically on the body, and given rectally. NUR4 reported his observations with topical applications. "They take herbal leaves and then they grind it and then they mix with water or something and then they place it around the child. And some also use this hard cream like Alcobalm [local ointment]". DR2 shared her experience regarding use of enemas: "when they are in pain they boil some herbs and give it to the child...they will give some enema before they come to the hospital especially those who have abdominal distention".

Regarding the use of mechanical measures, four professionals (NUR1, NURs3-5) had experiences of some patients "tying the affected part" and "putting weight" such as building blocks and stones on a painful body part. NUR3 explained that in the attempt: 
to reduce the pain, sometimes the patient's request that the part of the body where there is pain be tied up so that it will reduce the throbbing pain they feel. Or they will ask that a weight be put on that joint or that part or joint which again, sort of numbs the part of the body and reduces the pain that they are experiencing.

Other traditional practices reported involved use of surgico-spirituality. Two professionals (NUR2, NUR3) had experiences of patients believing their disease was caused spiritually. These patients sought spiritual interventions from medicine men or prayer centres. Medicine men usually treat such patients by scarification and insertion of medicines. NUR2 shared her experiences on how traditional medicine men remove disease causing spirits from SCD patients:

They have also what we call the scarification that are done by traditional medicine men with the belief that sickle cell disease is a bought disease, a spiritual disease and therefore by making the scarification on patients and putting in some black quotient can reduce the effects of the disease on the patients through evil manipulation. So they do the scarification and then they sort of give to the spirits. (NUR2)

In addition, the professionals reported their awareness of patients using conventional methods such as heat, pain medication and hydration especially during crisis. Several shared accounts of what patients revealed to them. For example DR1 noted:

When there is pain one woman will tell you when my child start complaining of pain I give the child brufen, I ask her...to take a lot of fluid and then I see what happens, if nothing happens fine, if she still complained then I rush the child here.

The respondents reported that some patients had their own ways to manage anaemia using natural or manufactured drugs and substances. Patients may prepare these medicines or remedies by themselves or purchase them over-the-counter. Natural haematinics are usually prepared from commonly used green vegetables. NUR2 cited her experience as follows:

And another person told me something about a drink that is made with 'Kwawunsusua' (Turkey Berries). She boils the Kwawunsusua, so that the water 
that will be used to boil is green and she drinks it with anything, like she adds sugar, if you want milk you add to make it nice. According to her that is her blood tonic so she takes it like tea every morning.

Synthetic haematinics may be bought from the pharmacy or from drug retailers. Patients were reported to be influenced to purchase these drugs based on the messages about their efficacy in producing blood. Though the professionals indicated little knowledge of the types of medicines patients buy; they suggested that these are usually processed medicines. Patients may take these medicines concomitantly with their prescribed medicines or may stop the prescribed medicines and use only the traditional medicine.

The health professionals reported advising patients to desist from using blood tonics, since these can lead to iron overload; causing problems for the patient:

We also discourage them because most blood tonics contain iron and therefore they may be adding more iron to what they already have because we know that when the cells are getting destroyed the iron is stored in their system for use. So if you have not done any test to actually show that they are lacking iron, then they are actually adding more iron in terms of taking the blood tonic. NUR1

According to the two doctors, patients usually consider fever as malaria and treat themselves accordingly at home. In the event of fever patients will only report to the hospital after unsuccessfully treating malaria. Though the professionals tended to agree that patient can treat for malaria at home when they experience fever, they also encourage them to report to hospital afterwards for confirmation and further management.

Regarding leg ulcers, three professionals (DR1, NUR1, NUR2) recounted that patients self-treat their ulcer because the chronic nature of the ulcers makes patients frustrated after prolong period of unsuccessful treatment at the hospital. People also self-treat because of the financial cost of hospital treatment. Professionals further indicated that some patients may never seek hospital treatment unless their ulcers become infected. With respect to how patients treat their ulcer, two professionals described equipment used to dress the ulcers and the method of dressing. Substances used to dress ulcers may include hot water or herbal medicines. Hot water is applied on the wound as lotion using a bucket as receptacle and a 
towel as the agent of application. Regarding using herbs to treat ulcers, NUR1 shared: They...pick leaves, herbs and then they grind them and apply them on the wound and some also use roots of a plant, grind them and apply them on the whole leg around the wound.

Self-treatment of other conditions was referred to infrequently. Concerning jaundice, NUR2 commented that patients consider this is a form of fever and manage it accordingly; usually with some unknown herbal medicine they buy from shops. NUR5 described how patient managed dactylitis at home: "We had a case where someone came with dactylitis and was using rope to tie the affected part. When we hear cases like that we take note them and use it to educate the association members against such practices" (NUR5).

\subsection{Managing Psychosocial Challenges}

Professionals commented on the psychosocial challenges confronting patients and the strategies they recommend to surmount them. The sources of psychosocial challenges were patient's domestic environment, disease symptomatology and community belief. Regarding domestic environment, four professionals (NURs2-4, NUR6) mentioned home conditions that may affect the care of a SCD child. They considered that the care the child receives in the household may become compromised if there are conflicts between spouses due to the SCD. If there is only one child with SCD, the child may face rejection by their siblings and other family members. Finances especially for single parents (usually the mothers) make it difficult to care for children. Nurses in particular advise families how to manage such issues.

Two professionals (NUR3, NUR6) said that a healthy physical environment promotes the health of the patient and reduces complications of the disease. Families are advised on what to do to ensure a healthy environment such as the following:

The room should be airy enough such that you wouldn't need to have air conditions. These days' patients are using sliding glasses and sliding whatever, I think it is not the best. The louvers should be opened the rooms should be well ventilated you know such that it is not stuffy. I mean these basic things are needed. A comfortable bed for the client. (NUR6)

With respect to disease symptomatology, professionals noted that frequent painful crisis leads to absenteeism from school and interferes with children and young people's 
education: "Some of them are discouraged...especially when they have crisis frequently and someway somehow they miss class, some of them are discouraged and would want to drop out. So...we encourage them [to stay in school]" (NUR2).

Many professionals revealed some community belief about SCD which impact impacts negatively on patients and families. These include supernatural causation, misconception, and labelling. Professionals noted that many patients believe SCD and related problems are caused by spiritual forces such as witches and patients who feel stigmatised by such belief may feel shy to disclose their status or seek treatment at the clinics:

There are some kind of misconceptions about SCD... and so patients with SCD are stigmatised and some of them don't understand the condition so...they attribute it to spiritual or supernatural reason...so it makes them not even seek treatment at all and patients will also not come to the hospital because of stigmatisation. Others even don't want to disclose their status. They don't want patients to even know that they have SCD. I myself I have a cousin who is hiding, even knowing that I am a nurse I thought that she will even confide in me so that I can give the necessary support but she doesn't want me to know the child has SCD just because patients will stigmatise. (NUR1)

In addition, professionals highlighted having experienced misconceptions about the genetic basis of SCD. Professionals indicated that some fathers of SCD children often blame mothers as the cause of the child's condition leading to marital disharmony and other family conflicts.

On the side of the parents sometimes they [mothers] are sacked from the house especially when they have problems with their husbands. Most of the time the husband thinks that it is the fault of the woman that the child is like that. Some of them claim their husbands leave them because of the child and some of them also come to realised that they got married to the wrong patients. (NUR2)

Professionals also mentioned that due to disease symptomatology, patients label SCD patients as weak patients; not worthy of anything good because they are candidates for early death. Children with SCD can be prevented from playing or other activities their counterparts do as a 
result of some of these labels. Such labels may result in adverse psychological effects such as hopeless, depression, rejection, and impaired development.

The professionals discussed the strategies they adopt to help patients to address these psychosocial challenges. Two professionals (NUR4, NUR1) provided ways they address community beliefs. Patients are advised to understand that SCD is treatable and early treatment is essential to reduce misconception; parents are advised to avoid the consequences of labelling and stigmatising their children; patients are also given words of encouragement. They are advised to cultivate self-confidence and engage in employment. These professionals thought these strategies contribute to reducing the negative connotations patients ascribe to the disease.

In regard to avoidance of stigmatisation, NUR6 advised parents as follows:

That the children need care and they should be stigmatized against because when they are stigmatized against, they have psychological trauma that can affect their growth and other things so usually, we educate the parents against some of these things...I will also educate that if they believe that they can live long and then they can do something themselves. So most of them we teach them to work, so in their management it is also good to accept the fact that they can also do something.

Furthermore, the professionals said they advised patients to collaborate and partner with health professionals and seek support from the Sickle Cell Association and Foundation. Male partners were advised to support their female counterparts in the care of their SCD children. Professionals who gave this advice referred to their previous experiences of complaints of conflicts they receive among parents.

Another strategy professionals' reported involved giving words of encouragement. Expressions such "don't give up" and "master courage" were used to urge patients and parents to persevere in the face of SCD related challenges. These were particularly in reference to patients who were in despair because of the chronicity of problems, complication of disease and cosmetic disfigurement:

Initially they had in mind that sickle cell they don't live for long, but now they've realised that it's not true. If you take good care of them they will survive because at 
the sickle cell clinic at Komfo Anokye [KATH] when I was part of it we used to have 60 years, 65 years university lecturers all coming for the clinic. They were a source of inspiration to the mothers who were around; so we tell them, "don't give up, don't give up". It will be surprising to know patients one month coming to hospital two times with severe haemolytic anaemia, then they worry about "why me"... why my child always coming to hospital for blood, then we tell them "don't give up". You can have two sickle cell patients in a family, one will be doing well, one will be going down, down, down...it doesn't matter, don't give up. So you don't have to give up, eat well, not just any meal. (DR1)

Regarding patients disturbed by cosmetic disfigurement, DR1 further had these words to encourage them:

Yes...always we tell them to master courage, I mean see them not different from the other children. If you look at... the children are always emotionally disturbed, why are my eyes always yellow? Why do I have bossing head? Why am I different from my siblings? So...parents should master the skill of explaining to the child that look you are not ill you are a well child, and then after 8, 9 years, you just tell them look, the reason why...treating you differently is that you are a sickle cell patient.

Another way professionals noted SCD patients can be encouraged to build emotional strength is for parents and significant others to show love to them. Adolescents and adult patients especially need to be shown lots of love and care since they are usually neglected by their friends. Showing love and care was linked with enhancing patient's immunity.

I think managing the emotional and social impact of the disease on that [SCD] child is very important especially for the teenagers and those in adulthood, they having friends, they living a normal life. These are neglected in a way but they should be looked at to help improve upon their health. Because basically health is not you having a disease or infirmity but it [is]... about your social, emotional aspect of your health so that can also boost your immune system. (NUR6)

Promoting emotional strength was considered important through general education and counselling. According to NUR3, promoting the ability to deal with the emotional 
consequences of the disease is the goal of education for parents with children with SCD. Adolescents are counselled to enable them to overcome challenges related to self-identity, education, psychological fears and schooling problems:

Now with the young adults...through with self-identity, challenges they go through with having to keep up academic work, the psychosocial challenge with having and maintaining friends and also at the same time looking attractive and then also going through these psychological fear of early death and struggling through leading an independent adult life. Yes so we teach them about how to keep...balance in school, when to learn and when not to learn, avoiding extremes of temperature, eating well, information to the teacher to know your condition well, in case you need support they can give to you, having access to water where you can have it as and when you need it. The need to visit the toilet regularly as and when it's needed. And then eating nutritious food that will help to keep the blood, the haemoglobin up. I think generally this is what I will say. (NUR3)

NUR5 mentioned that sometimes special programmes and camps are organised to educate teenagers and adult patients on marriage and relationship issues on ad hoc basis.

\subsection{Summary}

The health professionals' interviews revealed the various strategies they advise patients to adopt to manage their SCD in Ghana. Advice for preventive health includes use of frequent oral hydration, eating healthy diet, maintaining personal hygiene, and using supportive medication. It also includes avoiding extreme temperature, preventing dehydration, reducing over-activity, avoiding specific food, medicines, infections, injuries and unhealthy lifestyles. They also advised patients to consult and collaborate with their health professionals for regular checks, counselling and treatment for presenting problems. Self-monitoring advice involved regular checks for pallor, urine colour, fever, and jaundice. It also included splenic palpation, as well as observation of general demeanour and growth monitoring among children. Self-diagnosis advice concerned signs and symptoms that need urgent care. These were pallor, fever, urine colour changes, jaundice, unusual feelings, enlarge or tender spleen, prolong crying of children. Generally, patients were advised to report any atypical signs and symptoms. Regarding self-treatment, the professionals shared what they advise and what they 
have observed patients do. Pain management advice included use of analgesics, increased hydration, heat applications, and use of massage, relaxation and periodic movement. Fever management included antipyretics, tepid sponging, and increased fluids intake. Dactylitis management includes increased oral fluids, application of soothing creams, and use of analgesics. The professionals also revealed their experiences regarding how patients selfmanage common SCD and related problems including pain, anaemia, fever, leg ulcers, jaundice and dactylitis; using traditional and conventional measures.

The professionals noted a number of psychosocial challenges confronting SCD patients and strategies they use to assist patients. According to the professionals, psychosocial challenges emanated from patients' home environment, disease symptomatology and people's beliefs about SCD and related illnesses. Strategies the professionals reported using included giving words of encouragement, advice about availability of SCD treatment; importance of early treatment; the need for patients to engage in employment; seeking support and collaboration with health professionals, SCD foundation or Association; urging people to show love to the patients and public education about SCD. They also reported organising special education sessions to assist adolescence deal with their peculiar challenges.

Majority of the recommendations were for both adults and children but more child focused recommendations were located than adults. New variables identified in the professionals' interviews included addressing food misconceptions. Self-diagnosis indicated included change in demeanour, prolong crying, diarrhoea and vomiting, growth monitoring among children. Professionals also talked about self-treatment observations involving the use of traditional approaches including use of varieties of traditional medicines and other practices. These findings were added to the list of potential survey items and were also used to inform the interview guide for patients and parents 


\section{Chapter 8: Perspectives of Patients and Parents}

This chapter presents the results of interview with patients and parents of children with SCD. The first part of the result describes the socio-demographic characteristics of the participants. The second part presents the self-management actions reported by the participants. This part is organised into five sections including preventive health, selfmonitoring, self-diagnosis, and self-treatment. Part three focuses on psychosocial concerns raised by the participants that affect their self-management and general care, and the strategies participants adopt in addressing them.

\subsection{Socio-demographic Characteristics of the Participants}

The study involved 19 participants, including: 10 adult patients with SCD, 16 years and older, and nine parents of children ( $\leq 15$ years) with SCD. The socio-demographic characteristics of the participants are summarised in Tables 19 and 20. All except two parents/guardians were mothers. The ages of participants ranged from 28 to 72 years. The children concerned comprised of four females and five males with ages ranging from 3 to 15 years. The adult patient group comprised of four females and five males between the ages of 21 and 65 years. Individual parents are referred to as $\mathrm{CH} 1-\mathrm{CH} 9$ and adults as AD1-AD10. Where appropriate the participants are referred to as the adult group or the parent group. All the participants shared their strategies for self-management, however, the extent of their engagement with self-management varied.

\subsection{Preventive Health}

The findings show three areas of preventive health - routine health maintenance, preventive management, and consultations with health professionals.

\subsubsection{Health Maintenance}

Health maintenance actions were the strategies that patients took on a daily basis to keep well. Both the adults and parents spoke about two main categories of strategies they utilised for health maintenance - actions people took independently without consulting health professionals, and actions that were prescribed by health professionals. The former concerned hydration, nutrition, supportive medication, rest, personal hygiene and grooming. The latter 
included periodic medical checks, taking prescribed medications, prompt utilisation of health facilities for treatment of illnesses and complications. Hydration and nutrition were the major strategies the participants described.

TABLE 18. SOCIO-DEMOGRAPHIC CHARACTERISTICS OF PARENTS/GUARDIANS AND CHILDREN

\begin{tabular}{|c|c|c|c|c|c|c|c|c|c|}
\hline \multicolumn{6}{|c|}{ Parents/ Guardian Particulars } & \multicolumn{4}{|c|}{ Children Particulars } \\
\hline ID & Relationship & Age & Education & Employ & Residence & Age & Sex & Type & $\begin{array}{l}\text { Recruit } \\
\text { ment }\end{array}$ \\
\hline $\mathrm{CH} 1$ & Mother & 55 & Primary & Trading & Rural & 11 & $\mathrm{M}$ & $\mathrm{SS}$ & $\begin{array}{l}\text { Hospital/ } \\
\text { Phone }\end{array}$ \\
\hline $\mathrm{CH} 2$ & $\begin{array}{l}\text { Grand } \\
\text { Mother }\end{array}$ & 72 & None & Food vendor & City & 15 & $\mathrm{~F}$ & $\mathrm{SC}$ & Home \\
\hline $\mathrm{CH} 3$ & Mother & 45 & Tertiary & Teacher & Rural & 8 & $\mathrm{~F}$ & SC & Hospital/ \\
\hline $\mathrm{CH} 4$ & Mother & 40 & None & Farming & District & 12 & $\mathrm{M}$ & SS & $\begin{array}{l}\text { Hospital/ } \\
\text { Phone }\end{array}$ \\
\hline $\mathrm{CH} 5$ & Mother & 46 & Primary & Unemployed & District & 3 & $\mathrm{~F}$ & SS & Hospital \\
\hline CH6 & Mother & 28 & Primary & Trading & District & 8 & $\mathrm{~F}$ & SS & $\begin{array}{l}\text { Hospital/ } \\
\text { Phone }\end{array}$ \\
\hline $\mathrm{CH} 7$ & Anti & 31 & Tertiary & Teaching & District & 15 & $\mathrm{M}$ & $\mathrm{SC}$ & $\begin{array}{l}\text { Hospital/ } \\
\text { Phone }\end{array}$ \\
\hline $\mathrm{CH} 8$ & Mother & 37 & Primary & $\begin{array}{l}\text { Food } \\
\text { Vendor }\end{array}$ & City & 3 & $\mathrm{M}$ & SS & Hospital \\
\hline $\mathrm{CH} 9$ & Mother & 35 & Tertiary & Unemployed & City & 12 & $\mathrm{~F}$ & SS & Home \\
\hline
\end{tabular}

Note. Relationship=Relationship of guardian to a child; Employ=Employment of parent/guardian; Type= Type of SCD; M=Male; F=Female; Recruitment= Place of recruitment; Hospital= recruited face to face at the hospital; Hospital/phone= Recruited at the hospital through phone call; Home=Recruited from home through patient network.

TABLE 19. SOCIO-DEMOGRAPHIC CHARACTERISTICS OF ADULT PARTICIPANT

\begin{tabular}{|l|l|l|l|l|l|l|l|}
\hline & & & & & & & \\
\hline ID & Sex & Age & Education & Residence & Employment & Type & Recruitment \\
\hline AD1 & M & 65 & Primary & District & Trading & SC & Association \\
\hline AD2 & F & 29 & None & City & Food vendor & Not known & Associating/ Phone \\
\hline AD3 & F & 37 & Intermediate & City & Trading & SS & Association \\
\hline AD4 & F & 44 & Intermediate & City & Unemployed & SS & Association/snow \\
\hline AD5 & M & 27 & Tertiary & City & Trading & SC & Association/phone \\
\hline AD6 & F & 54 & Primary & District & Unemployed & SC & Clinic/Phone \\
\hline AD7 & M & 32 & Secondary & City & Draftsman & SS & Association \\
\hline AD8 & M & 24 & Secondary & District & Unemployed & SS & Clinic \\
\hline AD9 & M & 28 & Secondary & City & Unemployed & SS & Snowballing \\
\hline AD10 & F & 21 & Secondary & District & Unemployed & SS & Clinic/Phone \\
\hline
\end{tabular}

Note. Association=Recruited faces to face at the Sickle Cell Association Meeting; Association

Phone=Recruited from the Sickle Cell Association through phone call; Home=Recruited from home through patients' contact.

Most participants described hydration as a key strategy for achieving optimum health.

Both groups spoke about conscious efforts they make to frequently drink more fluids to hydrate their bodies. Participants commonly used expressions such as "lots of water" and 
"drink more" to illustrate their actions. Aspects of hydration participants commonly spoke about were types, quantity, factors influencing their fluid intake and precautions. The types of fluids mentioned were water and other liquids; water was the commonest. Whilst adults reported using mainly water, parents in addition to water gave their children other fluids such as natural or processed fruit juices, soft drinks, soups, and food beverages such as Milo. Only one adult (AD7) mentioned the occasional use of soft drinks. Both groups expressed caution with the use of very cold fluids such as "chewing ice block", "drinking ice water", and "water placed in fridge".

The quantity of fluid taken were reported in specific and non-specific terms and varied between adults and children. The parents reported giving their child 3000 to 4,500 millilitres (mls) a day, and adults reported taking 1000 to 7,500 mls a day. Both groups used the "voltic" water bottle (1.5 litres) or "sachet water" (300mls) for their measurement: "He can drink three of the 1.5L bottles of voltic water before night falls. I don't even have to give it to him, he takes the water himself; he drinks a lot of water" (CH8). The quantity of fluids parents gave was influenced by certain factors. Some parents reported increase fluid intake during hot weather, before checking children's urine, during the child's play time; and reduced in the evening (for children with enuresis.

Many participants also identified nutrition as an important self-management strategy. For most participants, the objective of eating was to promote health, or to prevent or treat an illness. Generally, the major objective of feeding among parents was to promote the health of their children whilst that of adults was to prevent crisis. Participants reported adopting three main strategies for meeting these nutritional objectives - food they endeavour to eat, the food they avoid or eat with caution, and cooking their own meals.

The choice of food nutrients patients endeavours to have was influenced by the perceived benefits of the food to their health. Parent's narratives indicated the use of a variety of diet strategies to promote their children's health and prevent diseases. For instance, parent (CH3) whose aim was to prevent SCD problems referred to giving food items such as fruit mixed with natural spices such as ginger and garlic to prevent arthritis and a calcium rich diet to prevent bone infection. Many parents tended to feed their children with the aim of 
promoting haematopoiesis. Such parents frequently demonstrated a physiological understanding between nutrition and haematogenesis.

I believe nutrition is a major factor when it comes to sickle clients. I am also aware that after 21days the blood of the sickle cell has to be replenished and so I make sure he eats an egg every three days. I also make sure he eats more vegetables; drink a lot of soup and eat liver. When he was a child, I used to mix most of his food with soya beans. This has been very helpful. (CH3)

One adult referred to eating specifically for these health benefits: At first I hardly ate kontomire and beans, in fact, I didn't like it at all but now I eat them because of my health (AD3). However, for $\mathrm{CH} 1$ her reasons for choice of food was based on the child's food preferences rather than health benefits.

Seven participants from both groups described food substances they avoid or eat with caution. The majority of these foods substances centred on the consumption of fats and oils. Although most of these participants did not provide specific oily food, two (CH5, AD8) specified groundnut (peanut) and one (AD5) referred to rice cooked with oil. The type of food avoided was informed by personal experiences, advice from health professionals and influenced by lay knowledge. In terms of personal experiences, most participants recounted experiencing crises after eating these foods. For example, AD3 mentioned having experienced a crisis after eating fufu (pounded cassava and plantain eaten with soup). Two patients (AD3, AD5) had also observed a particular time of the day when eating certain food resulted in a crisis:

I manage to avoid certain food that give me crises... rice cooked with oil, especially when I eat it in the evening... as I said earlier, I don't eat rice cooked with oil in the night and also make sure I do not overfeed myself. (AD5)

Apart from their personal experiences, $\mathrm{CH} 5$ and AD3 had each been advised by others or health professionals to avoid particular foods. In order to reduce or avoid exposure to oily foods, some patients cooked their own food and selected food that were low in fat.

Many participants expressed positive benefits of feeding on their health or their children's health. Some participants considered proper feeding as superior to using SCD- 
related medicines. In relation to this parent $\mathrm{CH} 3$ stated, "It is helping a lot more than even the medications being taken. The feeding and knowing how to handle her at home is very important in the management of SCD". For another parent $(\mathrm{CH} 7)$, the concern was that preparing special food for the child on a daily basis created a burden for the household.

As part of health maintenance both adults and parents reported use of supportive medication for their or their children's SCD care. Their narratives centred on the types of medicines, dosage, source, compliance and adherence issues. These medications included prescribed and not prescribed medications. Folic acid was the commonest medication referred to by both groups, but a few reported using penicillin V. Four parents routinely gave their child folic acid and parent $\mathrm{CH} 8$ also gave her child penicillin. Non-prescribed medications were usually bought over-the-counter, from drug retailers, from persona. Contacts and or through patients own adventure. Parent $\mathrm{CH} 1$ had given her child a trial of an unnamed food supplement to boost the immune system but stopped because her child developed rashes.

The parents and adults had different commitments to adhering to medication instructions. Parents tended to express their endeavours to adhere to medication instructions and avoided skipping daily doses: "I keep their drugs where their after-bath dressing things are. So I give them after their shower. Even in cases the drug is finished, I do well to buy some" (CH5). This endeavour to comply with medicines was influenced by the parent's knowledge about the functions of the medications: "I encourage her to take her folic acid daily, juice and water. The things that help in blood building, I encourage her to take those things" (CH9).

Similarly, of the six adults who mentioned using supportive medication; four took folic acid. However, unlike the parents, adults were less committed to adherence to the recommended regimen. For example, AD2 admitted skipping her medication sometimes due to laziness. AD1 only took it when he experiences a crisis. AD4 took up to 4 tablets of folic acid instead of one a day depending on his financial status. In addition to folic acid, AD4 referred to taking analgesic such as paracetamol and ibuprofen routinely, and AD9 regularly took multivitamins (B complex and Multivite) to stay fit.

The participants highlighted the importance of resting to their health. Different approaches were reported among children and adults. Resting among children involved 
parents having schedules for their children. $\mathrm{CH} 9$ for example, had a routine which involved her 12year old daughter resting two hours each afternoon. Parents were also conscious of the importance of their child having a "good" night's sleep. Parents with children with enuresis reported promoting a good night's sleep by periodically waking up the child to urinate, limiting fluid intake at night, providing comfortable bedding and a quiet sleeping environment. The adults mainly spoke about rest in connection with completing their daily activities safely.

\subsubsection{Consultations with Health Professionals}

In addition to what adults and parents do independently, a major strategy participants spoke about concerned, consultation with health professionals at the SCD clinic or the Association for periodic review, therapeutic care, education and counselling. The participants expressed different levels of engagement with the health professionals. Parent $\mathrm{CH} 4$ claimed she had sought clinical care for every problem before and after her child's diagnosis at aged 12 , and had never tried any self-treatment. This parent reported lack of awareness that she could self-treat some SCD problems. In contrast, $\mathrm{CH} 5$ rarely attended SCD clinics as she claimed to have an efficacious traditional medicine to treat her 3 year old child. In general, most participants indicated their endeavour to follow advice from health professionals.

Most participants considered their encounters with health professionals as positive. Many acknowledged that interactions with health professionals has resulted in the improvement of their condition, and has eased the burden of living or caring for SCD. Other benefits reported included reduction in the psychological burden of the disease; a sense of hope; reduction in frequency of illness; and the ability to manage minor conditions at home. Participants often made these observations when they compared their previous before encounter with health professionals and current health status or when their conditions was managed based on lay knowledge. One parent expressed her views as follows: "It's no more a difficult task for me [SCD care]. After what the woman [health professional] taught me, I don't even think about the disease anymore" (CH2). Similarly, AD1 who had suffered many complication from childhood and was only diagnosed with SCD in his adult life (aged 31years) shared: "Because I follow the advice of the doctor, am very healthy. Sometimes, I even think I don't have sickle cell. I fall sick once a year, particularly, during the cold season in December". 
In spite of these benefits, many participants commented on challenges of accessibility and affordability of SCD focused services. These concerns related to limited SCD clinics and services, their inability to afford transportation, and the limited coverage of the National Health Insurance Scheme (NHIS). One parent stated:

Some of the drugs prescribed are very expensive and outside the NHIS cover. When such drugs are prescribed for me it becomes very difficult for me to buy. It makes me feel that even if me as a government worker is facing this challenge, how those with menial jobs will be able to afford. (CH3)

Another parent (CH9) commented on the unfavourable clinic days (weekdays) that make it difficult for school children to utilise the clinics effectively. She recommended that the clinic days be extended to the weekends. Many adults recounted their frustration with KATH due to long waiting hours at the OPD, and the difficulties in getting physicians to see them.

At first we were all directed to one place for treatment and we were having our own doctor who was giving us treatment. But now it is not like that, you have to go to the hospital at dawn and join a very long queue before you get your treatment, meanwhile you will be going through the pains. (AD3)

The participants appealed to the Government to help resolve this situation. Regarding attendance at Association meetings, many participants reported they had never attended and others had discontinued attending. The reasons cited were: lack of financial support from the Association, work commitments, interferes with child school attendance as meetings are held on weekdays, and lack of finance for transportation.

\subsubsection{Preventive Management}

Preventive management captures actions that parents and adults performed with the primary objective of preventing or avoiding exacerbation of their disease. All participants referred to using at least one preventative action. Participants' responses were in reference to avoiding extremes of temperature, maintaining safety with activities; preventing malaria, and avoiding unhealthy lifestyles. The major focus of these concerned extremes of temperature and safety with activities. 
Twelve participants spoke about two strategies for avoiding extremes of temperature. The majority concerned avoiding cold and keeping warm. The strategies for avoiding cold differed between parents and adults. For parents, they involved avoiding consumption and bodily exposure to cold. Most parents ensured that their children avoided drinking or eating cold substances such as: drinking "iced water" and "chewing ice blocks"; bathing with cold water; and being exposed to rain especially in cold weather conditions. The adults' strategy mainly involved avoiding bodily exposures to cold such as staying away from cold places, touching cold objects; and avoiding walking in rains. All but one of the adults said they experienced crisis when exposed to extreme cold. AD3 however, had a different experience: "For me, I experience the crisis when the weather is hot. I am mostly okay when the weather is cold, but when it is warm, then I experience the crisis...I use cold water for washing".

The strategies for keeping warm were similar for both groups. Parents usually kept their child warm through the use of warm clothing and bathing with warm water. They also ensured that their children wore protective clothes under certain conditions such as cold weather, and periods of rain. Parent $\mathrm{CH} 9$ shared her experiences on keeping her 12 year old child warm during school days: "Anytime it rains, I make sure after she dresses, she puts on a jacket as well before she goes to school. I also warn her not to remove the uniform until the sun is scorching”. Three parents (CH2, CH6, CH9) ensured that their children bathed with warm or hot water irrespective of the weather conditions, and Parent $\mathrm{CH} 9$ only allowed her child to bath with tap water when the weather was extremely hot. Similarly, many adults kept warm by wearing warm clothes with long sleeves to cover the entire body, bathing with warm water, and sleeping with bed covers. Whilst the majority reported having warm baths AD9 shared a different strategy, "I was advised that, I should not allow myself to feel too hot or cold. So when the weather is hot, I bath cold water and when the weather is cold, I bath hot water".

Both groups mentioned the importance of activity safety to their health. Generally, participants used two key strategies to ensure safety - activities done with caution or moderation and activities avoided. The activities done with moderation or caution had different purposes for the two groups. For most parents, the main purpose was to enable their children to enjoy playing. The parents said they do not prevent their children from playing but guard the children to avoid exhaustion. The parents said they either stop their child when they 
believe the child has reached their energy limit or ask the child to drink alongside and continue. Apart from parent $\mathrm{CH} 9$ who referred to football, the remaining did not specify the particular play. Many parents reported that their current behaviour regarding their child's play was informed by advice from health workers:

I used to prevent him from playing but then when we went to the hospital, I was advised to allow him play, when he is tired, then I allow him to rest. So nowadays, I allow him to play, when he is tired, he comes to sit by me. (CH1)

In contrast, the type of activities the adult patients reported engaging were those that gave them some therapeutic effect. For AD3 and AD9 these activities involve deliberately engaged in household chores like sweeping; business activities. While for others it is gentle exercises that make them fit and reduces the frequency of illness.

Both groups, however, recounted similar types of activities they avoid - hard or strenuous activities. Whilst the parents said these within the context of play, adult said these within the context of work/occupation and sports. For the children, this included types of play they considered as hard or strenuous. For example, parents $\mathrm{CH} 1, \mathrm{CH} 3, \mathrm{CH} 4, \mathrm{CH} 7$, and $\mathrm{CH} 9$ did not allow their child to do sporting activities such as running, football, ampe (a vigorous traditional game) and jumping; domestic chores such as pounding fufu; lifting heavy objects; and entertainment such as dancing. Such decisions, tended to be influenced by previous experiences with painful crisis. Parent $\mathrm{CH} 9$ shared, "when she [daughter with $\mathrm{SCD}$ ] was 6 years... when she dances for an hour, she even complains of pains the next day". Likewise, the majority of the adult participants said they avoided activities they describe as "hard", "strenuous", or "tiresome work" and activities that require the use of much energy." Examples were farming, football, jogging, and fighting. AD6 stated:

I used to work very hard on my farm. However, I spent about two weeks in the hospital every month. So I stopped the farming and started hawking. Nowadays, because of my waist and joint pains, I don't do that anymore.

Another area of preventive management raised by some adult participants related to avoidance of unhealthy lifestyles. Their discussions centred on avoiding excessive sexual 
intercourse, alcoholism and overcrowding. Such decisions were informed by advice from health professionals or patients' own awareness of their physiological vulnerability.

All participants viewed malaria prevention as important in preventing crisis and anaemia. The interviews revealed that participants mainly prevent malaria through the use of physical barriers such as insecticide treated nets and for parents having their child wear protective clothing. AD9 spoke of wearing socks in the night. Participants usually talked about malaria in relation to previous experiences with illness. For example, parent CH3's decision to have her child sleep in a mosquito net was because her child's crises were frequently preceded by symptoms of malaria. Parent $\mathrm{CH} 4$ raised her concerned that her child refuses to sleep in the net during hot weather conditions.

Most participants viewed their preventive health actions as helpful. The adults and parents spoke of the benefits of using a balance diet, preparing fluids with local spices and vegetables, regularly taking folic acid, keeping warm, maintaining safe activity as helping them or their child keep fit and reducing the frequency of illness. For example, parent $\mathrm{CH} 3$ compared the use of medication and balanced diet as follows: "It is helping a lot more than even the medications being taken. The feeding and knowing how to handle her at home is very important in the management of SCD". However, two participants expressed concern that their poor financial status made it difficult for them to practice certain preventive actions such as purchasing warm clothes and eating good food and this impacted negatively on their health.

\subsection{Self-monitoring}

Participants reporting engaging in various forms of self-monitoring. The findings show that they engage in two types of self-monitoring - monitoring of physical and physiological abnormalities that serve as pointers of emerging problems, and monitoring for triggers of painful episodes and illnesses. The strategies they used for monitoring were observations and complaints. Most parents and adults reported mainly using observations. Six parents reported checking their children's eye frequently for changes in eye colour and swellings around the eyes. According to the parents changes in eye colour (yellow or red) or swellings can serve as cues for impending illness, usually, painful crisis. None of the adult patients mentioned self-monitoring using eye colour checks. 
The second commonest monitoring by participants was urine checks for changes in colour, such as brown, yellow or "Coca-Cola" urine. Parent CH9 shared how she monitors her daughter's urine for impending malaria and dehydration.

I have a routine check-up, when she wakes up, she drinks water and then, urinates so I check the colour... When the colour is negative, I tell her to take good care of herself at school, she should do well to take more water at school as well. When she comes back from school, I check her urine colour again to see how it looks...Even with the urine colour, even if it is deep it only becomes deeper when she has malaria. I check if it is concentrated, because I know every normal urine is light, however, when the colour is yellowish or brown, then it means there is a problem somewhere or there is dehydration. (CH9)

A few participants also reported checking for pallor, temperature and child's demeanour, and two parents also referred to monitoring their child's "blood supply" by observing for pallor in the lips, palms, and finger nails. AD9 was the only adult who referred to pallor checks: "I check my palm to check the colour, it's sometimes white”. Parents CH1 and $\mathrm{CH} 8$ referred to checking their child's temperature as a strategy for monitoring infection. Even though parent $\mathrm{CH} 7$ noted she did not engage in any monitoring of her child, she reported that she observed their child's general demeanour for unusual calmness and loss of weight. In addition to observed signs, parents took heed of the child's complaints such as headache, fatigue, and feverish feelings. Few adults spoke about monitoring using physical or physiological indicators, instead they spoke about monitoring in terms of observing bodily sensations or cues such as fatigue, which can be signified by continuous yawning and stretching.

Self-monitoring also included the exploration of triggers for disease exacerbation. Two types of triggers were reported; those related to exposures and those related to skipping certain procedures. The participants reported being watchful of triggers of disease named under preventive care such as temperature levels and types of activities, and being careful to perform actions required to promote their health such as eating or taking the "right" food or medications, and treating infections promptly. Many participants reported having experienced disease exacerbation when they did not keep tract of these actions. 


\subsection{Self-Diagnosis}

The analysis revealed the use of various medical signs and symptoms as indicators to recognise SCD problems that needed intervention. These indicators were substantive for the organ on which the signs were recognised or pointers to problems in another organ. Regarding symptoms, painful body parts was the most frequent diagnostic indicator referred to by both groups. Adults indicated that any part of the body could be affected, but for children, their limbs (legs, hands, arms) were affected the most. A few parents also specified knee joints and the back. Parents usually diagnosed their younger children through palpation of the body following prolonged crying. In comparison, older children would verbalise or complain to their parents. Other minor symptoms parents mentioned were itchy eyes, stomach pains, constipations, severe weakness, and difficulty in walking.

Most adults reported having experienced severe bodily pain without mentioning specific body parts. Three adults, however indicated their waist, heels, hands and joints were commonly affected. Other symptoms reported by adults were pointers to apparent illnesses, usually painful crisis. These included frequent yawning and feeling of the urge to stretch in the morning, feeling unwell, bitterness in the mouth, rapid heartbeat, inability to walk and painful eyes. Three adult males reported priapism as an indication of ill health. AD9 who has recurrent priapism expressed his frustration as follows: "Priapism, It is now my major problem. I really suffer when it starts and I wish they can even castrate me". Adults AD6 and AD7 also mentioned pallor in the eyes and palms as indicators of a shortage of blood.

The parent participants also reported using signs to identify SCD related problems. More parents reported using signs than adults. The parents described their innovative ways of detecting or forecasting illness among their children. The commonest of these were eye colour changes. According to the parents, red eyes indicated that the child had a crisis, yellow eyes indicate malaria or feeling sick, and white or pale eyes indicated shortage of blood. Other parents used urine colour changes as diagnostic signs. For example, according to $\mathrm{CH} 9$, yellow urine indicates jaundice, brown indicates dehydration, and "deep" urine indicates malaria. The other signs were fever, prolong erection of the penis and abdominal swelling. Parent CH8, uses a temperature level of $37^{\circ} \mathrm{C}$ (measured by thermometer) as her criteria for determining fever in her child, whilst another parent $\mathrm{CH} 4$ referred to using touch. One parent $(\mathrm{CH} 1)$ 
reported she recognises that her son is about to experience crisis when he experiences priapism in the morning after urinating.

\subsection{Self-Treatment}

Participants described a number of approaches they utilise for therapeutic care. The approaches involved general and specific self-treatment strategies. They referred to actions targeted at curing the disease and of aborting the entire associated symptomatology whilst the specific referred to actions targeting at particular problems.

Both adults and parents reported using traditional or conventional strategies for selftreatment. The majority of these related to use of traditional strategies of which traditional medicines were the commonest. The traditional medicines used were in the form of raw herbs, or locally processed herbs or alternative medicine. More adults reported having used traditional medicines for this purpose than parents. However, a few participants reported being current users of traditional medicines. Of the five parents who reported giving their children traditional medicines to treat or cure SCD, only two were current users. These participants reported giving the medicines in different forms. For example, parent $\mathrm{CH} 2$, reporting using concoction prepared from "Nkrampan" [a plant] for the child to drink in order to minimise the disease. Parent $\mathrm{CH} 5$ said she barely attends the hospital; she employs a combination of herbs and conventional medicine to treat every problem the child develops. She explained her actions as follows:

When she [SCD child] falls sick, the first thing I do is to give her the herb...there is one called "Ferma" used for the soup, "Tuwentini" which I use in bathing them and they also drink some. "Aguro" I grind and boil it for them to drink...my mum gives me some herbs which I use to prepare soup. When they take the soup, they urinate and sweat a lot. They become well in some few days.

The two current traditional medicine users expressed more confidence in herbal medicines than conventional ones in managing general symptoms. In relation to this $\mathrm{CH} 5$ stated:

Formerly, when she becomes sick, it takes time before she becomes fine, however, because of the herbs I give her, she becomes well within two days. Those [earlier] 
days, when you see her physically, she looks soft and dull, but now, she has become very strong.

Whilst $\mathrm{CH} 3$ decision to use herbs was entirely based on its efficacy, $\mathrm{CH} 5$ 's decision was partly due to financial difficulties in affording hospital treatment. Whilst three parents $(\mathrm{CH} 3$, $\mathrm{CH} 5, \mathrm{CH} 6)$ considered traditional medicines useful, the majority reported otherwise. Many had vowed never to use them again: "Yes, my mother took me to a herbal clinic [name withdrawn] but it didn't help, she took me to other places but it never helped. So I have made up my mind to go nowhere apart from the hospital" (AD9).

Similarly, most adults reported having been exposed to using herbal medicines and alternatives including prayers to manage their condition. All but AD6, were given the medicines by their parents when they were younger and most could not remember the names. These medicines were taken orally, mixed with food or applied topically. For example, AD7 shared how traditional medicines were applied topically to treat his SCD:

When I was around 7 years, they put a hot stone into water and mixed it with the water in a banana sucker. They use it to smear my leg and they tie it with a scarf and they put an ice block on it but it never worked.

AD6 was the only current adult user of dompe-dompe (ointment prepared from herb) which she found useful in treating her pains. Apart from the herbal medicines, AD2 had also been treated with an alternative Chinese medicine to cure the disease which he did not consider was effective.

The participants also described strategies they adopt to manage specific problems, including pain, fever, priapism, headache, eye problems, stomach problems, constipation, malaria, common cold, anaemia, leg ulcers, and hip problems. Many spoke about pain management. Both adults and children used medicinal and non-medicinal interventions to treat their pain. Medicinal interventions used included oral and topical medicines from conventional or traditional sources. Regarding conventional medicines, both groups reported frequently using one or more medicines to treat their painful episodes. Medicines commonly administered orally were multivitamins including folic acid, B complex, and multivite, analgesics, including paracetamol, tramadol, and anti-inflammatory drugs including 
ibuprofen, diclofenac and Midol (paracetamol, caffeine and mepyramine combination). Participants used combinations of medicines from the multivitamin and analgesic groups or combinations of medicines from the same group. These practices tended to be more common among the adults. For instance AD3 used a combination of folic acid and ibuprofen, and AD4 took two analgesics (paracetamol and ibuprofen) simultaneously. AD1 and AD2 reported using only folic acid to treat their pain. AD4 reported using some unspecified pain killers he described as "Poku pharma", "Danny pharma" and "Kenapharma” drugs (Poku pharma, Danny, Kenapharma pharma are pharmaceutical agencies in Ghana). He also reported using some medicines with the inscriptions "CUS" and "SDS" donated by a friend from the USA. He claimed the medicines have analgesic and sedation functions. Five parents mentioned the use of similar conventional medicines of which folic acid was the commonest. Three participants used folic acid in combination with either ibuprofen or paracetamol. Parent $\mathrm{CH} 8$ however used penicillin to treat her child's pain.

In addition to oral medicines, both parents and patients frequently used topical medicines to treat their pain. Mostly, these medicines were used solely or with heat application. Topical medicines mentioned by the parents (five parents) were mainly of traditional source, including Shea butter, nompe-nompe, and lord balm. The adults (five adults) on the other hand reported use of both conventional and traditional topical creams. Conventional medicines reported were diclofenac gel and "rubb" (mentholated ointment). The traditional medicines named were "ice cool cream" "apomden", "lord balm" and "dompedompe ointment." Many expressed a preference for "hot" (harsh) ointments and others prefers medicines which contains menthol.

The commonest non-medicinal strategies for pain management involved the application of heat and increased intake of oral fluids. Five parents and six adults reported having applied heat to affected parts using hot water, towels and topical analgesics. Of the five parents, four found the intervention effective in reducing the pains, but the fifth parent (CH1) reported the application of heat increased her child's pain; hence she refrained from using it. Participants also reported using hydration and nutrition to manage painful crisis. Regarding the use of hydration, the participants referred to increasing their oral fluids. They used expressions such as "much water", "lots of water", without specifying quantity. One 
parent expressed fear about the side effects of medication and thought diet and nutrition were more effective than analgesics for managing SCD crisis.

I give him nutritious foods such as Milo drink and soft drinks. He loses appetite when he is in crisis, but he to take malt. I also make him take a lot of water. Other foods I give include eggs, and light soup, which encourages him and makes him happy... but it requires boldness to handle it. When they are in crisis, one would always give paracetamol but I have heard that too much use of paracetamol causes liver problems...Intake of soups and stews must also be regular. $(\mathrm{CH} 3)$

Two participants use mechanical methods. $\mathrm{CH} 3$ reported tightly holding the child's painful part for the pain duration and AD7 applied a bandage to the affected part.

Apart from pain management, the participants also discussed their strategies for managing specific SCD related problems. Four parents $(\mathrm{CH} 1, \mathrm{CH} 5$; CH6; CH8) spoke about how they manage their child's fever. All except CH5 used paracetamol or tepid sponging. However, CH5 would normally give medications that had previously been given from the hospital or purchase some over-the-counter. None of the adults reported fever management.

The three male adults described how they manage their priapism using pharmacological and non-pharmacological measures. AD9 was the only one who referred to use of pharmaceutical measures. He reported having experienced a reduction in the frequency of his priapism after using a medication termed "fruzemide" which he bought over-thecounter. He had previously used pain medication such as ibuprofen and paracetamol without success. However, all three men used non-pharmacological measures such as high fluid intake (about 7 voltic bottles), taking short walks and riding bicycle. AD9 non-pharmacological strategy for managing recurrent priapism which usually occurred in the night:

When I wake up from my sleep due to the pains it comes with, I take a cold bath [or shower] as I shower down, I jump like am exercising. It starts coming down and the pains go through am still erected. I tie a cloth around my waist without the cloth touching my penis. I walk around the hall till it calms down all by its self. Your penis starts erecting without you having any sexual intentions in mind. 
Only one parent, $\mathrm{CH} 1$ said her child experiences priapism but she always sought hospital treatment without trying any self-treatment

A few parents shared their strategies for managing their child's stomach pains and constipation. Parents $\mathrm{CH} 3$ and $\mathrm{CH} 4$ reported using herbal medicine, whereas $\mathrm{CH} 9$ used conventional medicines. Patient $\mathrm{CH} 3$ explained how she uses a herb to treat stomach pains and constipation: "the herbs "Nunum" in the form of an enema to treat the stomach ache...is very good and our grandmothers used it to treat abdominal pains. When I use it, she [child] gets instant relief from constipation and she feels better". Another parent, $\mathrm{CH} 9$ also explained how she uses conventional medicines to treat stomach pains:

She used to complain about her stomach, so I decided to always have Buscopan [Hyoscine butylbromide] or Trixillicate around. When she starts complaining the pain is around the navel. I give her Buscopan but any other part [body], I give her Trixillicate. I hardly give her brufen. It's normally, Buscopan, trixillicate and paracetamol. I only add the brufen in case the pain is extreme.

In addition, parent $\mathrm{CH} 9$ often gives the child a teaspoon of olive oil and fluids when she experiences constipation. $\mathrm{CH} 9$ and $\mathrm{CH} 3$ found their treatment strategies effective.

Regarding headache, two parents revealed how they managed with traditional medicines. Both reported applying herbs to the head. Although $\mathrm{CH} 1$ found the herbs useful, she stopped using them as the medicine man whom he consulted for the herbs insinuated metaphysical cause for the child's problems; which she disagreed.

Participants also made minor reference to the management of eye problems, malaria, common cold, anaemia, leg ulcers and hip problems. The participants reported using mainly conventional medicines for these problems. Two parents had used eye-drops, bought over-thecounter to treat their child's itching eyes without success. Parent $\mathrm{CH} 9$ also commented that she treats her child for common cold and malaria. Whilst she mentioned the use of antimalarial without specifying the particular type, she threw more light on common cold treatments: "I have some drugs, but I give her [daughter] more fruits and rich vitamin C before I give her the drugs. My basis is the fluids, I give her pineapple juice, sometimes, and I add ginger to the juice. She likes mangoes as well" (CH9). 
Parent $\mathrm{CH} 9$ mentioned used combined fluids, heat, massage and antibiotics to treat bodily weakness and difficulty in walking for her 12 year old daughter. Parents $\mathrm{CH} 4$ and $\mathrm{CH} 6$ referred to the use of conventional medicines to treat the shortage of blood when they detect pallor. While $\mathrm{CH} 4$ frequently gives blood tonics, $\mathrm{CH} 6$ gives multivitamins including B complex and folic acid. Both parents purchase these medicines over-the-counter.

Adult AD4 with chronic leg ulcer uses hot water and normal saline mix with "wokadin" to dress his wounds, and insufflate with metronidazole powder. His decision to self-treat his ulcer resulted from the poor attitude of health professionals and high cost of hospital treatment. He also reported injecting pethidine on his own due to the pain from the leg ulcer. AD8 however, uses hot compress and "hot ointment" to treat hip problems. According to him the intervention helped to reduce the pain.

The participants also revealed medicines and other logistic they keep in their homes for their self-treatment. Most participants mentioned having conventional medicines, mostly pain medications (ibuprofen, ointments, paracetamol), multivitamins (folic acid, B complex, Zincovit); and penicillin V. Two parents, however had herbs they use to add to their children food.

\subsection{Managing Psychosocial Concerns}

The participants' reports highlighted various psychosocial concerns patients and parents encounter and strategies adopted. These concerns related to disease symptomatology, care and treatment cost, and community perceptions.

Adults concerns were mostly related to disease symptomatology and that of parents were mostly financial, and getting their children to conform to therapeutic and behaviour change instructions. The adults revealed the impact of disease symptomatology on their lives. According to five adults, SCD interferes with their reproductive, relational, occupational, and educational lives. For instance, two females (AD3, AD6) shared that the challenges they could potentially encounter with pregnancy and labour resulted in their decision not to have children. Also cosmetic issues such as lean body and yellow eyes made some adults limit mingling with friends, or participating in entertainment, resulting in loneliness. According to these adults, the unpredictable nature of a crisis, prolonged anaemia, and frequent 
hospitalisation resulted in frustration. Three male adults were concerned that SCD renders them physically weak, making it difficult for them to engage in income generating employment, resulting in poverty, with associated emotional turmoil. For the children, two parents $(\mathrm{CH} 1, \mathrm{CH} 4)$ were concerned that prolonged illness from SCD could prevent their child from attending school for several weeks.

Both parents and adults indicated the impact of negative community beliefs about SCD. The dominant belief was related to the ascription of SCD association with metaphysical causes, including witches, demons and devils. These beliefs affected most adults and all but one parent (CH1). Although, the beliefs affected parents in several ways, their major concern were the discrimination and psychological trauma that goes with it. Though most parents had not been confronted directly with derogatory labels, people usually harass them with advice to seek traditional and spiritual avenues for cure such as prayer camps and traditional medicine men. Parents who got frustrated by such pronouncements spoke of hiding their children's SCD status. However, the participants noted that being well educated; accepting the scientific basis of SCD; seeking early treatment; receiving support from health professionals; and praying to God enhances effective coping:

Yes, it is true and so it is difficult to let others know your child have SCD. I have heard about the fact that people refer to the disease as something given by witches and wizards. It is a very big challenge because if you are not careful your child may be discriminated against. However, because of how parents are traced by the hospital staff for education it has helped to curb some of these misconceptions and myths. (CH3)

Most adult participants also shared they were aware of the perceptions of metaphysical attributes of SCD. However, contrary to the situation of parents, most adults had encountered derogatory labels which had resulted in negative consequences. The labels included being blamed as a wizard; being a candidate for premature death (up to 20 years); being a "Sickler" one who is almost always sick; having a "bought disease"; and being a person who causes financial drain. One adult shared his ordeal as follows:

some people say the disease is "abcwe cash" [financial drain] on parents or patients. This is because, you will spend all the money you make on drugs. Back at 
school, other school mates will not allow you to play with them because you have a sickle cell...even my mum ...told me that I am a financial drain on her. I felt really sad because I did not expect my mother to say that to me. I was really disturbed to the extent that, I felt she was not my real mother so I told my father to take me to my real mother. (AD7)

As a result of these beliefs, some participants reported experiencing negative repercussions usually from close relatives including parents, siblings and uncles. People shunned their company, rejected them and looked down upon them or ridiculed them. Consequently, patients may withdraw from public company or refuse to make friends. A participant had for example experienced expulsion from school because their uncle who was a caretaker did not want to spend his "hard earned money on a person who will die by age 20" (AD8). A female participant who faces a threat of divorce because of her SCD shared: Because of that [SCD], my husband hardly spends money on me. Some relatives even wanted him to divorce me but because of Christ, we are still together (AD6). Parent CH3, also expressed concern about poor paternal support for the care of her SCD child due to the misconception that the mother was the cause of the child's SCD.

Participants reported adopting various strategies to deal with psychosocial issues. The major strategy used involved self-confidence. Most participants tended to derive their confidence from availability of treatment for SCD (both bio-medical and traditional medicines), religion comparison with other SCD children and role models. Participants whose confidence was in bio-medical treatment tended to demonstrate a good understanding of SCD, and appreciated that although it is incurable, the disease can be adequately management to promote wellbeing. Two parents expressed they no longer worry about their child's condition due to the high efficacy of the herbal treatment they give their children: "When I came to believe in the herbs, I gave it to her [SCD child] and I have realized that, it is helpful. Because of this, am not really worried when am not able to take the girl [SCD child] to the hospital” (CH5).

Confidence derived from religion was expressed by two adults and two parents all Christians. These participants believed in the continuous sustenance of God despite their illness. Adult AD6 hopes for a healing from God; "I know nothing is difficult with God. I am 
just hoping and praying that in God's own time he should take this disease away from me." Two other participants derived their confidence from comparing their condition to other SCD patients. CH9 compared her child's situation to another SCD child in her neighbourhood who had worse disease symptomatology. She was encouraged that her child's condition was not as bad as the other children. AD2 was encouraged by a role model who has successfully lived with SCD to old age:

One of the Association members also encouraged us at one of our meetings. He said he has sickle cell disease and he is 60 years going to pension so it's never true that sickle cell patient die at the age of 20. (AD4)

Two adults (AD2, AD3) also expressed they have few worries about living with their disease as a result of support they receive from their parents. Some parents also spoke about strategies for helping their children in school to cope emotionally with SCD. Three parents talked about interactions with their child's school teachers. These interactions were usually informed by the parents' experience of some inappropriate handling of their children in school. For instance, parent CH4's child had been beaten severely by a teacher which resulted in the child been sick for a long time. Another parent, whose child was in a boarding house said her child did not have warm water, special food and other basic logistics required to keep well. These parents spoke of the importance of collaborating with teachers. Parent $\mathrm{CH} 7$ shared that he addressed this by taking a letter to the school from health professionals with the intention that his son would be given some exceptional treatment.

Many participants identified that knowledge of SCD and self-management was important for their coping. The majority reported gaining their knowledge from health professionals at the SCD clinic and the Sickle Cell Association. Although many viewed the education they have received from health professionals as limited, they acknowledged that the knowledge they received has been helpful in effectively coping with their condition.

\subsection{Summary}

Self-management strategies reported in this chapter parallels what was reported in the health professional's interviews, but the content of the narratives differed in some areas. In general, adults and parents identified similar areas of self-management, but the frequency of 
strategies used differed among the groups in several areas. Few strategies were also peculiar to each of the groups. The most common actions reported were hydration, nutrition, avoidance of extremes of temperature, avoidance of over activity, and the use of folic acid supplementation. Regarding self-monitoring and diagnosis, parents place emphasis on observable indicators compared to the adults who used more subjective indicators. Patients identified their own strategies for interpreting signs and symptoms. The patients also enumerated strategies used to manage pain, fever, leg ulcers, priapism, headache, and infections including malaria. These strategies were added to the item list and contributed in informing the survey questionnaire. The next chapter reports the results of the survey of the parents and patients regarding their health and self-management actions. 


\section{Chapter 9: Quantitative Survey}

The survey results are presented in six parts. Part one presents the socio-demographic and clinical characteristics of the respondents, and their major SCD problems experienced during the 12 months preceding data collection. Part two presents the self-management actions patients and parents reported for managing SCD. It begins with descriptive summaries of the total number of self-management actions individuals reported, followed by a description of the four main self-management areas (preventive health, self-monitoring, selfdiagnosis, and self-treatment). Part three presents the evaluation of participant's self-treatment actions. Part four focuses on self-reporting of health status and QOL of participants. Part five concerns the results of self-management resources used for managing SCD. Part six presents identifies concerns related to SCD.

Data is presented in tables to compare adults and children and are ranked to facilitate comparison. Data is presented as count, percentage, mean, standard deviation, range and pvalues where appropriate. Significant $\mathrm{p}$ - values ( $\mathrm{p}$ less than or equal to 0.05 ) are bolded in the tables. Unless otherwise stated in the table notations, $\mathrm{p}$ - values are based on Chi-square test for independence between groups (adult/child) and the action. Only "yes" responses are presented. Percentages may total more than $100 \%$ allowing for multiple selection of actions.

\subsection{Description of the Sample}

The sample comprised of 313 respondents of which 112 (35.8\%) were adult SCD patients and $201(64.2 \%)$ were parents of SCD children (Table 21). The sample obtained corresponds to $74 \%$ of the calculated sample size of 422 . The sample reported selfmanagement for 204 children and 109 adults with SCD (Table 22). Of the 204 children selfmanagement responses, $196(96.1 \%)$ parents provided the information. The $8(3.9 \%)$ children who self-reported the survey were aged 13-15 years. Of the 109 adult responses, 104 (95.6\%) adult patients self-reported. Five (4.4\%) of the adult patients aged 16-19 were unavailable and their parents completed the survey.

As adults and children with SCD have differences regarding SCD management the data is analysed by child and adult recipients of care, rather than who provided information. In 
this chapter "adults" refers to SCD patients 16 years and older and "children" refers to SCD patients 15 years and younger whose self-management were reported.

\subsubsection{Socio-Demographic Characteristics of the Sample}

Table 20 shows the characteristic of parents who reported on behalf of their SCD children, and adults SCD patients who reported for themselves. Mothers were the majority of parents fathers, relatives and children themselves. The majority of parents and adult patients had attended school, but approximately one tenth, mostly parents, had no formal education or completed primary or intermediate level schooling.

The distribution of employment status shows that slightly more than half were selfemployed (artisans, business, trading) and more than a tenth (13.8\%), slightly more among the adult patients, were unemployed. almost all participants received no or low monthly income ( $\leq \mathrm{GH} \mathbf{C} 899$ ), and were most common among the Adults group. Approximately two thirds of respondents reported spending up to one half or more of their income on SCD care and approximately a tenth did not know how much they spent on SCD care. Almost all respondents attend the SCD clinic with most children were seen 3-4 times in the previous 12 months. The SCD clinic was the only health service some respondents attended. The majority travelled for up to one hour to reach their nearest SCD clinic. Those who accessed other services, utilised public hospitals for other health care and five respondents attended homeopathic clinics.

Table 22 illustrates the socio-demographic characteristics of the adults and children. The mean age of the sample was 13.65 \pm 11.65 years, range 1-61 years. Among children, the majority $(162,81.4 \%)$ were between 1 to 10 years while approximately two thirds of the adults were between 16 to 24 years. There were fewer males (145) than females (168) in the total sample. The distribution of males and females and type of SCD differed significantly between adults and children as indicated by the p-values. Among the children, males constituted a majority (51.5\%) but females were the majority in the adult sample. Patients with $\mathrm{HbSS}$ and $\mathrm{HbSC}$ constituted the majority (82.1\%) of the sample. The adult group had a higher proportion of $\mathrm{HbSS}(58.7 \%)$ than the children $(48.5 \%)$ whilst the children group had a slightly higher proportion $(30.3 \%)$ of $\mathrm{HbSC}(29.4 \%)$. A few $(8.9 \%)$ of the respondents did not know their genotype and this was more common in the children's group. 
TABLE 20. SOCIO-DEMOGRAPHIC CHARACTERISTICS OF RESPONDENTS

\begin{tabular}{|c|c|c|c|c|}
\hline Characteristics & Value & $\begin{array}{l}\text { Total } \\
n=313(100 \%)\end{array}$ & $\begin{array}{l}\text { Adults } \\
\mathrm{n}=112(35.8 \%)\end{array}$ & $\begin{array}{l}\text { Parents } \\
n=201(64.2 \%)\end{array}$ \\
\hline $\begin{array}{l}\text { Relationship to the } \\
\text { child }\end{array}$ & $\begin{array}{l}\text { Mother } \\
\text { Father } \\
\text { Relative } \\
\text { Patient* }\end{array}$ & $\begin{array}{r}150(47.9) \\
27(8.6) \\
19(6.1) \\
117(37.4)\end{array}$ & 112 & $\begin{array}{r}150(74.6) \\
27(13.4) \\
19(9.5) \\
5(2.5)\end{array}$ \\
\hline $\begin{array}{l}\text { Highest school } \\
\text { completed }\end{array}$ & $\begin{array}{l}\text { None } \\
\text { Primary } \\
\text { Intermediate } \\
\text { Secondary } \\
\text { Tertiary }\end{array}$ & $\begin{array}{l}34(10.9) \\
88(28.1) \\
90(28.8) \\
68(21.7) \\
33(10.5) \\
\end{array}$ & $\begin{array}{r}5(4.5) \\
15(13.4) \\
32(28.6) \\
42(37.5) \\
18(16.1)\end{array}$ & $\begin{array}{c}29(14.4) \\
73(36.3) \\
58(28.9) \\
26(12.9) \\
15(7.5)\end{array}$ \\
\hline Parents occupation & $\begin{array}{l}\text { Artisan } \\
\text { Business } \\
\text { Government } \\
\text { Private salary } \\
\text { Unemployed } \\
\text { Student } \\
\text { None }\end{array}$ & $\begin{array}{r}30(9.6) \\
104(33.2) \\
29(9.3) \\
41(13.1) \\
35(11.2) \\
59(18.8) \\
15(4.8)\end{array}$ & $\begin{array}{r}10(8.9) \\
13(11.6) \\
11(9.8) \\
15(13.4) \\
10(8.9) \\
53(47.3) \\
0(0.0)\end{array}$ & $\begin{array}{r}20(10.3) \\
91(45.3) \\
18(9.0) \\
26(12.9) \\
25(12.4) \\
6(3.0) \\
15(7.4)\end{array}$ \\
\hline Marital status & $\begin{array}{l}\text { Single } \\
\text { Married } \\
\text { Divorced } \\
\text { Widowed } \\
\text { Relationship }\end{array}$ & $\begin{array}{r}99(31.6) \\
183(58.5) \\
16(5.1) \\
9(2.9) \\
6(1.9)\end{array}$ & $\begin{array}{r}81(72.3) \\
26(23.2) \\
3(2.7) \\
1(0.9) \\
1(0.9)\end{array}$ & $\begin{array}{r}18(9.0) \\
157(78.1) \\
13(6.5) \\
8(4.0) \\
5(2.5)\end{array}$ \\
\hline $\begin{array}{l}\text { Monthly income in } \\
\text { GHc }\end{array}$ & $\begin{array}{l}\text { None } \\
\text { Less than } 100 \\
100-499 \\
500-899 \\
900+\end{array}$ & $\begin{array}{r}112(35.8) \\
49(15.7) \\
97(31.0) \\
37(11.8) \\
18(5.8) \\
\end{array}$ & $\begin{array}{r}64(57.1) \\
17(15.2) \\
17(15.2) \\
9(8.0) \\
5(4.5)\end{array}$ & $\begin{array}{r}48(23.9) \\
32(15.9) \\
80(39.8) \\
28(13.9) \\
13(6.5)\end{array}$ \\
\hline $\begin{array}{l}\text { Proportion of income } \\
\text { spent on SCD care }\end{array}$ & $\begin{array}{l}\text { Just a little } \\
\text { Almost half } \\
\text { More than half } \\
\text { Almost all } \\
\text { All of income } \\
\text { Don't know } \\
\text { NA* }\end{array}$ & $\begin{array}{r}91(29.1) \\
63(20.1) \\
22(7.0) \\
10(3.2) \\
8(2.6) \\
37(11.8) \\
82(26.2)\end{array}$ & $\begin{array}{r}18(16.1) \\
20(17.9) \\
7(6.2) \\
4(3.6) \\
0(0.0) \\
15(13.4) \\
48(42.9)\end{array}$ & $\begin{array}{r}73(36.3) \\
43(21.4) \\
15(7.5) \\
6(3.0) \\
8(4.0) \\
22(10.9) \\
34(16.9)\end{array}$ \\
\hline $\begin{array}{l}\text { Ownership of health } \\
\text { insurance }\end{array}$ & $\begin{array}{l}\text { Yes } \\
\text { No }\end{array}$ & $\begin{array}{r}303(96.8) \\
10(3.2)\end{array}$ & $\begin{array}{r}104(92.9) \\
8(7.1)\end{array}$ & $\begin{array}{r}199(99.0) \\
2(1.0)\end{array}$ \\
\hline $\begin{array}{l}\text { Attendance to SCD } \\
\text { care }\end{array}$ & $\begin{array}{l}\text { Yes } \\
\text { No }\end{array}$ & $\begin{array}{r}290(92.7) \\
23(7.3)\end{array}$ & $\begin{array}{r}100(89.3) \\
12(10.7)\end{array}$ & $\begin{array}{r}190(94.5) \\
11(5.5)\end{array}$ \\
\hline $\begin{array}{l}\text { Other health services } \\
\text { used for SCD care }\end{array}$ & $\begin{array}{l}\text { Public } \\
\text { Private } \\
\text { Homeopathy } \\
\text { None** }\end{array}$ & $\begin{array}{r}104(33.2) \\
17(5.4) \\
5(1.6) \\
187(59.7)\end{array}$ & $\begin{array}{r}33(29.5) \\
3(2.7) \\
1(0.9) \\
75(67.0)\end{array}$ & $\begin{array}{r}71(35.3) \\
14(7.0) \\
4(2.0) \\
112(55.8)\end{array}$ \\
\hline $\begin{array}{l}\text { Travel time to nearest } \\
\text { SCD clinic }\end{array}$ & $\begin{array}{l}<30 \text { minutes } \\
\text { 30-59minutes } \\
\text { 1-3hours } \\
\text { 4-7hours }\end{array}$ & $\begin{array}{r}71(22.7) \\
152(48.6) \\
85(27.2) \\
5(1.6)\end{array}$ & $\begin{array}{r}31(27.7) \\
47(42.0) \\
31(27.7) \\
3(2.7)\end{array}$ & $\begin{array}{r}40(19.9) \\
105(52.2) \\
54(26.9) \\
2(1.0)\end{array}$ \\
\hline
\end{tabular}

*Students or unemployed respondents. ** Respondents who have never attended any other health services except SCD clinic. 
TABLE 21. SOCIO-DEMOGRAPHIC CHARACTERISTICS OF ADULTS AND CHILDREN GROUPS

\begin{tabular}{|c|c|c|c|c|c|}
\hline Characteristics & Value & $\begin{array}{c}\text { Total } \\
n=313(100 \%)\end{array}$ & $\begin{array}{c}\text { Adults } \\
\mathrm{n}=109(34.8 \%)\end{array}$ & $\begin{array}{c}\text { Children } \\
\mathrm{n}=204(65.2 \%)\end{array}$ & $\mathbf{P}$ \\
\hline Gender* & $\begin{array}{r}\text { Male } \\
\text { Female }\end{array}$ & $\begin{array}{l}145(46.3) \\
168(53.7)\end{array}$ & $\begin{array}{l}40(36.7) \\
69(63.3)\end{array}$ & $\begin{array}{r}105(51.5) \\
99(48.5)\end{array}$ & 0.017 \\
\hline Age & $\begin{array}{r}1-5 \\
6-10 \\
11-15 \\
16-24 \\
25-34 \\
35+\end{array}$ & $\begin{array}{r}91(29.1) \\
71(22.7) \\
42(13.4) \\
64(20.4) \\
24(7.7) \\
21(6.7)\end{array}$ & $\begin{array}{r}0(0.0) \\
0(0.0) \\
0(0.0) \\
64(58.7) \\
24(22.0) \\
21(19.3)\end{array}$ & $\begin{array}{r}91(44.6) \\
71(34.8) \\
42(20.6) \\
0(0.0) \\
0(0.0) \\
0(0.0)\end{array}$ & NA \\
\hline Type of SCD** & $\begin{array}{r}\text { HbSS } \\
\text { HbSC } \\
\text { HbSThal } \\
\text { Other } \\
\text { Don't know }\end{array}$ & $\begin{array}{c}163(52.1) \\
94(30.0) \\
10(3.2) \\
18(5.8) \\
28(8.9)\end{array}$ & $\begin{array}{r}64(58.7) \\
32(29.4) \\
5(4.6) \\
1(0.9) \\
7(6.4)\end{array}$ & $\begin{array}{r}99(48.5) \\
62(30.4) \\
5(2.5) \\
17(8.3) \\
21(10.3)\end{array}$ & $<0.034$ \\
\hline
\end{tabular}

\subsection{Major Problems Patients experienced in the Past 12 months}

Table 22 illustrates the distribution of problems experienced by patients in the previous 12 months. Overall, painful crisis was the most common (59.4\%) through to sudden seizures, the least common (1.3\%). The proportion of people who experienced individual problems differed considerably between the two groups. Whilst jaundice emerged as the commonest reported problem for adults $(65.5 \%)$, painful crisis was the commonest among children (58.5\%). Statistically significant differences were observed in jaundice, fever, sudden weakness, hip problems and priapism. Adults experience jaundice (65.1\% vs $45.6 \%)$, sudden weakness (32.1\% vs $14.7 \%)$, hip problems (28.4\% vs $6.4 \%)$, and, for male patients, priapism ( $25 \%$ vs $6.7 \%$ ) more frequently than children. In contrast, children were more likely to experience fever $(56.4 \%$ vs $42.2 \%)$.

\subsubsection{Frequency of crisis}

Table 23 presents the frequency with which patients had experienced painful crises in the last 12 months. Approximately half (54\%) of the respondents reported having experienced a crisis between one and eight times. Approximately a third (30.7\%) did not report experiencing a painful crisis and this was slightly more common among adults.

Approximately one in 50 respondents could not recall the number of times a painful crisis was experienced. More children experienced a crisis nine or more times than adults $(19.1 \% \mathrm{vs}$ $1.8 \%)$. These differences were statistically significant $\left(\chi^{2}=34.153, \mathrm{df}=5, \mathrm{p}<.001\right)$. 
TABLE 22. PROBLEMS PATIENTS HAVE EXPERIENCED IN THE LAST 12MONTHS

\begin{tabular}{|c|c|c|c|c|c|c|c|}
\hline & & Total & Adu & & Child & & \\
\hline & Problem & n (\%) & n (\%) & Rank & n (\%) & Rank & p \\
\hline 1. & Painful crisis & $186(59.4)$ & $67(61.5)$ & 2 & $119(58.3)$ & 1 & 0.677 \\
\hline 2. & Jaundice & $164(52.4)$ & $71(65.1)$ & 1 & $93(45.6)$ & 3 & $<0.001$ \\
\hline 3. & Fever & $161(51.4)$ & $46(42.2)$ & 4 & $115(56.4)$ & 2 & 0.023 \\
\hline 4. & Severe headache & $145(46.3)$ & $55(50.5)$ & 3 & $90(44.1)$ & 4 & 0.341 \\
\hline 5. & Shortage of blood & $81(25.9)$ & $25(22.9)$ & 6 & $56(27.5)$ & 5 & 0.463 \\
\hline 6. & Sudden weakness & $65(20.8)$ & $35(32.1)$ & 5 & $30(14.7)$ & 7 & $<0.001$ \\
\hline 7. & $\begin{array}{l}\text { Abdominal } \\
\text { swelling }\end{array}$ & $62(19.8)$ & $24(22.0)$ & 8 & $38(18.6)$ & 6 & 0.570 \\
\hline 8. & Hip problems & $44(14.1)$ & $31(28.4)$ & 7 & $13(6.4)$ & 8 & $<0.001$ \\
\hline 9. & Priapism* $(n=145)$ & $17(11.7)$ & $10(25.0)$ & 9 & $7(6.7)$ & 10 & 0.005 \\
\hline 10 & Leg ulcer & $16(5.1)$ & $8(7.3)$ & 10 & $8(3.9)$ & 9 & 0.299 \\
\hline 11 & Sudden seizures & $4(1.3)$ & $3(2.8)$ & 11 & $1(0.5)$ & 11 & NA \\
\hline
\end{tabular}

TABLE 23. FREQUENCY OF CRISES PATIENTS HAVE EXPERIENCED IN THE PAST 12 MONTHS

\begin{tabular}{|l|r|r|r|}
\hline Frequency & $\begin{array}{r}\text { Total } \\
\mathbf{n = 3 1 3}\end{array}$ & $\begin{array}{r}\text { Adults } \\
\mathbf{n = 1 0 9}\end{array}$ & $\begin{array}{r}\text { Children } \\
\mathbf{n = 2 0 4}\end{array}$ \\
\hline None & $96(30.7)$ & $43(39.4)$ & $53(26.0)$ \\
\hline $1-4$ & $149(47.6)$ & $49(45.0)$ & $100(49.0)$ \\
\hline $5-8$ & $20(6.4)$ & $11(10.1)$ & $9(4.4)$ \\
\hline $9+$ & $41(13.3)$ & $2(1.8)$ & $39(19.1)$ \\
\hline Don't remember & $7(2.2)$ & $4(3.7)$ & $3(1.5)$ \\
\hline
\end{tabular}

\subsection{Self-Management Actions}

Table 24 presents the summary of the number of self-management actions respondents reported they used in the past 12 months based on pre-determined actions. The mean number of self-management action per respondent was 21.9 (range 11-36). People reported using more preventive health actions than other actions.

TABLE 24. SUMMARY SELF-MANAGEMENT ACTIONS BY PARTICIPANTS

\begin{tabular}{|l|r|r|r|}
\hline Actions & \multicolumn{1}{|c|}{$\begin{array}{c}\text { No. Actions } \\
\text { investigated }\end{array}$} & Mean (SD) & \multicolumn{2}{|c|}{ Range } \\
\hline Preventive health & 17 & $12.2(2.5)$ & $5-17$ \\
\hline Self-monitoring & 8 & $5.7(2.3)$ & $0-10$ \\
\hline Self-diagnosis & 11 & $3.6(2.4)$ & $0-11$ \\
\hline Self-treatment & 18 & $0.3(0.9)$ & $0-6$ \\
\hline Total & $\mathbf{5 4}$ & $\mathbf{2 1 . 9 ( 4 . 4 )}$ & $\mathbf{1 1 - 3 6}$ \\
\hline
\end{tabular}


Table 25 presents a comparison of the self-management actions reported in Table 25, sub-grouped by participant characteristics. Children used significantly more self-management actions than adults $(\mathrm{t}(311)=4.078, \mathrm{p}<0.001)$. Although the remaining sub-groups showed differences in the mean numbers of self-management actions, the differences were not statistically significant.

TABLE 25. COMPARISON OF SELF-MANAGEMENT ACTIONS BY PARTICIPANT'S CHARACTERISTICS

\begin{tabular}{|c|c|c|c|c|}
\hline & & Mean (SD) & Range & $\mathbf{P}$ \\
\hline \multirow[t]{2}{*}{ Adult/child } & Adult & $20.6(3.9)$ & $20.0(12-32)$ & \multirow[t]{2}{*}{$<0.001$} \\
\hline & Child & $22.7(4.4)$ & $25.0(11-36)$ & \\
\hline \multirow[t]{2}{*}{ Gender } & Male & $22.5(4.5)$ & $25.0(11-36)$ & \multirow[t]{2}{*}{0.914} \\
\hline & Female & $21.5(4.2)$ & $20.0(12-32)$ & \\
\hline \multirow[t]{2}{*}{ Residence } & Urban & $21.9(4.4)$ & $21.0(11-32)$ & \multirow[t]{2}{*}{0.969} \\
\hline & Rural & $22.0(4.3)$ & $24.0(12-36)$ & \\
\hline \multirow{2}{*}{$\begin{array}{l}\text { Access to } \\
\text { clinic }\end{array}$} & Yes & $22.0(4.4)$ & $25.0(11-36)$ & \multirow[t]{2}{*}{0.747} \\
\hline & No & $21.7(4.2)$ & $17.0(13-30)$ & \\
\hline \multirow[t]{4}{*}{ Genotype } & $\mathrm{HbSS}$ & $22.0(4.4)$ & $21.0(11-32)$ & \multirow[t]{4}{*}{0.820} \\
\hline & $\mathrm{HbSC}$ & $22.0(4.2)$ & $20.0(12-32)$ & \\
\hline & HbSthal & $21.5(4.3)$ & $12.0(15-27)$ & \\
\hline & Other & $22.8(3.7)$ & $13.0(17-30)$ & \\
\hline \multirow{4}{*}{ No of crisis } & Don't know & $21.2(5.4)$ & $23.0(13-36)$ & \multirow{4}{*}{0.314} \\
\hline & None & $21.6(4.2)$ & $21.0(11-32)$ & \\
\hline & $1-5$ times & $22.3(4.7)$ & $25.0(11-36)$ & \\
\hline & 6 more & $21.8(3.7)$ & $14.0(15-29)$ & \\
\hline \multirow[t]{5}{*}{ Education } & None & $21.6(4.5)$ & $18.0(11-29)$ & \multirow[t]{5}{*}{0.203} \\
\hline & Primary & $22.8(4.5)$ & $20.0(12-32)$ & \\
\hline & Intermediate & $21.8(4.7)$ & $24.0(12-36)$ & \\
\hline & Secondary & $21.1(4.0)$ & $19.0(13-32)$ & \\
\hline & Tertiary & $22.2(3.8)$ & $18.0(12-30)$ & \\
\hline \multirow[t]{6}{*}{ Income } & Less than 100 & $22.1(4.2)$ & $16.0(13-29)$ & \multirow[t]{6}{*}{0.790} \\
\hline & $100-499$ & $22.4(4.1)$ & $20.0(11-31)$ & \\
\hline & $500-899$ & $22.1(4.1)$ & $15.0(15-30)$ & \\
\hline & 900-1299 & $20.8(5.2)$ & $18.0(12-30)$ & \\
\hline & $>200$ & $20.2(5.7)$ & $15.0(15-30)$ & \\
\hline & None & $21.7(4.6)$ & $25.0(11-36)$ & \\
\hline
\end{tabular}

Note. P-values based on independent sample t-test for two group and one way ANOVA for multi-groups

The next section focuses on the detailed results of the self-management actions. All tables have columns that compare adults and children. Where appropriate, chi-square, test of independence or goodness of fit values are presented. The table includes columns which have ranked the results. Significant p-values are bolded and "NA" is used to indicate when 
statistical tests are inapplicable. Actions that involve consultation with health professionals were the majority but were not included in the ranking since the focus is on self-management.

\subsubsection{Preventive Health and Maintenance}

Table 26 and 27 show the results of patients' reported actions for preventive health and maintenance. Table 26 compares regular and periodic use of 17 pre-determined actions. Regularity was defined by regular use (daily or weekly) and periodic use (monthly or when necessary). Of the 17 preventive action areas, higher frequencies were recorded for regular use for 10 items (folic, lots of water, blood tonics, avoid cold baths, avoid cold weather, avoid cold drinks, take vitamins, sleep under mosquito nets, avoid strenuous activities and keep vaccination up-to-date) compared to periodic use. In contrast, higher frequencies were recorded for periodic use compared with regular use on six actions (dress to stay warm, use mosquito spray, avoid worrying, malaria prophylaxis, special diet, herbal medicines). In addition, to what is reported in the table, $181(57.8 \%)$ of children were reported to be up to date with their vaccinations (vaccination was only included in the children questionnaire). Significant differences were recorded between regular and periodic used actions on all items except three (blood tonic, avoiding cold weather, special diet). The overall ranking indicates that the top five regularly actions were folic acid $(n=304)$, lots of water $(n=298)$, sleep under mosquito nets $(n=173)$, avoid cold baths $(n=162)$ and take blood tonic $(n=145)$. On the other hand, the top five periodic used actions were worm medication $(n=224)$, dress to stay warm $(n=173)$, use of mosquito spray $(n=127)$, use of malaria prophylaxis $(n=123)$, avoid worrying $(n=112)$.

A comparison of the actions shows that the most two highly ranked items (folic acid, lots of water) were similar for both groups. Use of worm medication, was ranked the least within both groups. The remaining items showed differences in ranking between the two groups. A significant difference was observed between the two groups regarding their use of periodic and regular actions on six actions (dressing to stay warm, blood tonics, avoiding cold baths, avoiding cold weather, avoiding cold drinks, avoid strenuous activities). Children reported higher frequencies for regular actions including use of folic acid, blood tonic, avoid cold baths, avoid cold weather, avoid cold drinks, and avoid strenuous activities. In contrast, adults reported higher frequencies for periodic actions including to stay warm, and attend clinical appointments. 
TABLE 26. SUMMARY OF FREQUENCY OF PREVENTIVE HEALTH ACTIONS

\begin{tabular}{|c|c|c|c|c|c|c|}
\hline Action & $\mathrm{n}=313(\%)$ & $\begin{array}{r}\text { Regular } \\
\text { use }\end{array}$ & Rank & $\begin{array}{r}\text { Periodic } \\
\text { use }\end{array}$ & Rank & $\mathbf{p}$ \\
\hline Folic acid & $308(98.4)$ & $304(98.7)$ & 1 & $4(1.3)$ & 17 & NA \\
\hline Lots of water & $303(96.8)$ & $298(98.3)$ & 2 & $5(1.7)$ & 16 & $<0.001$ \\
\hline Dress to stay warm & $285(91.1)$ & $112(39.3)$ & 10 & $173(60.7)$ & 2 & $<0.001$ \\
\hline Blood tonic & $254(81.2)$ & $145(57.1)$ & 5 & $109(42.9)$ & 6 & 0.028 \\
\hline Avoid cold baths & $244(78.0)$ & $162(66.4)$ & 4 & $82(33.6)$ & 9 & $<0.001$ \\
\hline Avoid cold weather & $233(74.4)$ & $133(57.1)$ & 8 & $100(42.9)$ & 7 & 0.066 \\
\hline Worm medication & $227(72.5)$ & $3(1.3)$ & 17 & $224(98.7)$ & 1 & NA \\
\hline Avoid cold drinks & $227(72.5)$ & $143(63.0)$ & 6 & $84(37.0)$ & 8 & $<0.001$ \\
\hline Use mosquito spray & $218(63.9)$ & $91(41.7)$ & 12 & $127(58.3)$ & 3 & $\mathbf{0 . 0 1 0}$ \\
\hline Take vitamin & $201(64.2)$ & $143(71.1)$ & 6 & $58(28.9)$ & 12 & $<0.001$ \\
\hline Sleep under mosquito net & $200(63.9)$ & $173(86.5)$ & 3 & $27(13.5)$ & 15 & $<0.001$ \\
\hline Avoid worrying & $190(60.7)$ & $78(41.1)$ & 13 & $112(58.9)$ & 5 & 0.006 \\
\hline Strenuous activities & $180(57.5)$ & $110(61.1)$ & 11 & $70(38.9)$ & 10 & 0.004 \\
\hline Malaria prophylaxis & $134(42.8)$ & $11(8.2)$ & 16 & $123(91.8)$ & 4 & $<0.001$ \\
\hline Special diet & $80(25.6)$ & $38(47.5)$ & 14 & $42(52.5)$ & 13 & 0.823 \\
\hline Herbal medicine & $54(17.3)$ & $19(35.2)$ & 15 & $35(64.8)$ & 14 & $\mathbf{0 . 0 2 0}$ \\
\hline
\end{tabular}

Note. P-values are based on chi square test for goodness of fit comparing periodic and regular use among the actions

**Only included in children questionnaire. 
TABLE 27. FREQUENCY OF PREVENTIVE HEALTH ACTIONS

\begin{tabular}{|c|c|c|c|c|c|c|c|c|c|c|}
\hline \multirow[b]{2}{*}{ Preventative Health Action } & \multirow{2}{*}{$\begin{array}{l}\text { Total } \\
\mathrm{n}=313(\%)\end{array}$} & \multicolumn{3}{|c|}{ Regular user } & \multicolumn{6}{|c|}{ Periodic user } \\
\hline & & Adult & Rank & Children & Rank & Adult & Rank & Children & Rank & $\mathbf{p}$ \\
\hline Take Folic acid & $308(98.4)$ & $104(96.3)$ & 1 & $200(100)$ & 1 & $4(3.7)$ & 14 & $0(0.0)$ & 15 & $\mathbf{0 . 0 2 7}$ \\
\hline Take lots of water & $303(96.8)$ & $103(98.1)$ & 2 & $195(98.5)$ & 2 & $2(1.9)$ & 15 & $3(1.5)$ & 14 & NA \\
\hline Dress to stay warm & $285(91.1)$ & $27(28.7)$ & 9 & $85(44.5)$ & 8 & $67(71.3)$ & 2 & $106(55.5)$ & 2 & 0.015 \\
\hline Take blood tonic & $254(78.3)$ & $32(42.7)$ & 8 & $113(63.1)$ & 4 & $43(57.3)$ & 5 & $66(36.9)$ & 6 & 0.004* \\
\hline Avoid cold baths & $244(78.0)$ & $43(53.8)$ & 4 & $119(72.6)$ & 3 & $37(46.2)$ & 8 & $45(27.4)$ & 8 & 0.006* \\
\hline Avoid cold weather & $233(74.4)$ & $35(43.8)$ & 6 & $98(64.1)$ & 6 & $45(56.2)$ & 4 & $55(35.9)$ & 7 & $0.005 *$ \\
\hline Worm medication & $227(72.5)$ & $1(1.2)$ & 15 & $2(1.4)$ & 15 & $80(98.8)$ & 1 & $144(98.6)$ & 1 & NA \\
\hline Avoid cold drinks & $227(72.5)$ & $36(46.8)$ & 5 & $107(71.3)$ & 5 & $41(53.2)$ & 6 & $43(28.7)$ & 9 & $<.001 *$ \\
\hline Use mosquito spray & $218(63.9)$ & $26(33.8)$ & 10 & $65(46.1)$ & 10 & $51(66.2)$ & 3 & $76(53.9)$ & 5 & 0.105 \\
\hline Take vitamin supplement & $201(64.2)$ & $46(64.8)$ & 3 & $97(74.6)$ & 7 & $25(35.2)$ & 11 & $33(25.4)$ & 11 & 0.191 \\
\hline Avoid thinking & $190(60.7)$ & $18(36.7)$ & 11 & $60(42.6)$ & 11 & $31(63.3)$ & 10 & $81(57.4)$ & 4 & 0.586 \\
\hline Avoid strenuous activities & $180(57.5)$ & $35(49.3)$ & 6 & $75(68.8)$ & 9 & $36(50.7)$ & 9 & $34(31.2)$ & 10 & 0.014* \\
\hline Malaria prophylaxis & $134(42.8)$ & $7(14.9)$ & 14 & $4(4.6)$ & 14 & $40(85.1)$ & 7 & $83(95.4)$ & 3 & 0.081 \\
\hline Special diet & $80(25.6)$ & $16(48.5)$ & 12 & $22(46.8)$ & 12 & $17(51.5)$ & 13 & $25(53.2)$ & 12 & 1.000 \\
\hline Herbal medicine & $54(17.3)$ & $12(38.7)$ & 13 & $7(30.4)$ & 13 & $19(61.3)$ & 12 & $16(69.6)$ & 13 & 0.733 \\
\hline
\end{tabular}

Note. P-values are based on chi-square test of independence for association between adult/ child and periodic/regular use 


\subsubsection{Self-Monitoring}

The eight main reported self-monitoring strategies for both groups, in order of magnitude, are shown in Table 28. Urine colour and jaundice checks were the most common (ranked 1\&2). Pallor check was amongst the top five ranked actions for both groups. There were considerable variations in rankings for the remaining strategies. Children were significantly more likely than adults to report looking for pallor $\left(\chi^{2}=4.310, \mathrm{df}=1, \mathrm{p}=0.038\right)$ and keeping diaries $\left(\chi^{2}=153.86, \mathrm{df}=1, \mathrm{p}<.001\right)$. In addition to the variables illustrated in the table, children reported observing behaviour (167, 53.4\%), and monitoring growth (155, $49.5 \%)$. Of the 109 adults, $90(28.8 \%)$ reported listening to their bodies.

TABLE 28. SELF-MONITORING STRATEGIES

\begin{tabular}{|l|r|r|r|r|r|r|}
\hline Strategy & \multicolumn{1}{c|}{$\begin{array}{c}\text { Total } \\
\mathbf{n}(\boldsymbol{\%})\end{array}$} & $\begin{array}{c}\text { Adult } \\
\mathbf{n}(\boldsymbol{\%})\end{array}$ & Rank & $\begin{array}{c}\text { Children } \\
\mathbf{n}(\boldsymbol{\%})\end{array}$ & Rank & \multicolumn{1}{c|}{ P } \\
\hline Checks urine colour & $264(84.3)$ & $94(86.2)$ & 1 & $170(83.3)$ & 1 & 0.610 \\
\hline Checks for jaundice & $246(78.6)$ & $81(74.3)$ & 2 & $165(80.9)$ & 2 & 0.228 \\
\hline Look for pallor & $217(69.3)$ & $67(61.5)$ & 3 & $150(73.5)$ & 4 & $\mathbf{0 . 0 3 8}$ \\
\hline Keeps a diary/rec & $171(54.6)$ & $7(6.4)$ & 8 & $164(80.4)$ & 3 & $<\mathbf{0 . 0 0 1}$ \\
\hline Palpate abdomen & $125(39.9)$ & $39(35.8)$ & 4 & $86(42.2)$ & 5 & 0.392 \\
\hline Checks temperature & $86(27.5)$ & $30(27.5)$ & 5 & $56(27.5)$ & 7 & NA \\
\hline Looks for diarrhoea & $83(26.5)$ & $23(21.1)$ & 6 & $60(29.4)$ & 6 & 0.146 \\
\hline Observes for pain & $31(9.9)$ & $15(13.8)$ & 7 & $16(7.8)$ & 8 & 0.141 \\
\hline
\end{tabular}

\subsubsection{Triggers of crisis}

Table 29 presents the major triggers of crisis. Although exposure to cold weather or places recorded the highest value in the table (19.8\%), cumulatively, triggers related to cold exposure in general (cold bath, swimming, drinks or water, weather or places and exposure to rain) accounted for approximately one third (31.3\%) of responses. The other common causes reported were malaria and mosquitoes related (11.8\%). Both groups reported being similarly affected by all the triggers except exercise and strenuous activities where adults were twice as likely as the children to be affected $\left(\chi^{2}=6.860, \mathrm{df}=1, \mathrm{p}=0.009\right)$. 
TABLE 29. TRIGGERS OF PAINFUL CRISIS

\begin{tabular}{|l|r|r|r|r|r|r|}
\hline Cause & \multicolumn{1}{|c|}{$\begin{array}{c}\text { Total } \\
\mathbf{n}(\boldsymbol{\%})\end{array}$} & \multicolumn{1}{c|}{$\begin{array}{c}\text { Adults } \\
\mathbf{n}(\boldsymbol{\%})\end{array}$} & \multicolumn{1}{c|}{ Rank } & $\begin{array}{c}\text { Children } \\
\mathbf{n = ( \% )}\end{array}$ & \multicolumn{1}{c|}{ Rank } & \multicolumn{1}{|c|}{ p } \\
\hline Cold weather or place & $62(19.8)$ & $25(22.9)$ & 2 & $37(18.1)$ & 2 & 0.387 \\
\hline Exercise/hard work & $50(16.0)$ & $26(23.9)$ & 1 & $24(11.8)$ & 3 & $\mathbf{0 . 0 0 9}$ \\
\hline Malaria & $21(6.7)$ & $7(6.4)$ & 4 & $14(6.9)$ & 5 & 1.00 \\
\hline Exposure to rain & $19(6.1)$ & $3(2.8)$ & 6 & $16(7.8)$ & 4 & NA \\
\hline $\begin{array}{l}\text { Mosquitoes bites/not } \\
\text { sleeping under nets }\end{array}$ & $16(5.1)$ & $6(5.5)$ & 5 & $10(4.9)$ & 6 & NA \\
\hline Cold drinks/cold water & $10(3.2)$ & $3(2.8)$ & 6 & $7(3.4)$ & 7 & NA \\
\hline Cold bath or swimming & $7(2.2)$ & $1(0.9)$ & 11 & $6(2.9)$ & 8 & NA \\
\hline Eating certain food & $5(1.6)$ & $2(1.8)$ & 10 & $3(1.5)$ & 10 & NA \\
\hline Shortage blood/pallor & $5(1.6)$ & $3(2.8)$ & 6 & $2(1.0)$ & 13 & NA \\
\hline Refusing to eat & $4(1.3)$ & $1(0.9)$ & 11 & $3(1.5)$ & 10 & NA \\
\hline Physical trauma & $3(1.0)$ & $0(0.0)$ & 13 & $3(1.5)$ & 10 & NA \\
\hline Fever & $2(0.6)$ & $0(0.0)$ & 13 & $2(1.0)$ & 13 & NA \\
\hline Crying & $1(0.3)$ & $0(0.0)$ & 13 & $1(0.5)$ & 15 & NA \\
\hline Refusing to take medication & $1(0.3)$ & $0(0.0)$ & 13 & $1(0.5)$ & 15 & NA \\
\hline Can't tell & $63(20.1)$ & $21(19.3)$ & 3 & $42(20.6)$ & 1 & 0.897 \\
\hline Other & $8(2.6)$ & $3(2.8)$ & 6 & $5(2.5)$ & 9 & NA \\
\hline
\end{tabular}

\subsubsection{Self Diagnosis}

The indicators people reported using to recognise painful crisis are presented in Table 30. Pain was ranked as the most frequent affecting more than a third of adults (36.7\%) and children $(35.8 \%)$. Lower frequencies were recorded for the remaining indicators. The two groups showed a significant difference in relation to experience of fever $\left(\chi^{2}=5.22, \mathrm{df}=1\right.$, $\mathrm{p}=.022)$, and weakness $\left(\chi^{2}=6.860, \mathrm{df}=1, \mathrm{p}=.009\right)$. The Children group were approximately two and half times as likely as the Adult group to report fever as an indicator for crisis (16.2\% vs $6.4 \%$ ). Adults were approximately three times more likely than the children group to experience body weakness ( $15.6 \%$ vs $5.9 \%$ ) when crisis occurs. 
TABLE 30. INDICATORS FOR RECOGNISING PAINFUL CRISIS

\begin{tabular}{|l|r|r|r|r|r|r|}
\hline Indicator & \multicolumn{1}{c|}{$\begin{array}{c}\text { Total } \\
\mathbf{n}(\boldsymbol{\%})\end{array}$} & $\begin{array}{c}\text { Adults } \\
\mathbf{n}(\boldsymbol{\%})\end{array}$ & \multicolumn{1}{c|}{$\begin{array}{c}\text { Ran } \\
\mathbf{k}\end{array}$} & $\begin{array}{c}\text { Children } \\
\mathbf{n}(\boldsymbol{\%})\end{array}$ & Rank & p \\
\hline Pain & $113(36.1)$ & $40(36.7)$ & 1 & $73(35.8)$ & 1 & 0.971 \\
\hline Fever & $40(12.8)$ & $7(6.4)$ & 5 & $33(16.2)$ & 2 & $\mathbf{0 . 0 2 2}$ \\
\hline Weakness & $29(9.3)$ & $17(15.6)$ & 2 & $12(5.9)$ & 6 & $\mathbf{0 . 0 0 9}$ \\
\hline Prolong crying* & $27(8.6)$ & $2(1.8)$ & 10 & $25(12.3)$ & 3 & $\mathrm{NA}$ \\
\hline Loss of appetite/refusing to eat & $24(7.7)$ & $6(5.5)$ & 6 & $18(8.8)$ & 4 & 0.407 \\
\hline Jaundice & $16(5.1)$ & $4(3.7)$ & 7 & $12(5.9)$ & 6 & 0.564 \\
\hline Change in demeanour* & $16(5.1)$ & $1(0.9)$ & 13 & $15(7.4)$ & 5 & NA \\
\hline Swelling of body & $10(3.2)$ & $2(1.8)$ & 10 & $8(3.9)$ & 10 & NA \\
\hline Urine colour change & $10(3.2)$ & $4(3.7)$ & 7 & $6(2.9)$ & 11 & 0.991 \\
\hline Eye colour changes & $6(1.9)$ & $2(1.8)$ & 10 & $4(2.0)$ & 12 & NA \\
\hline Pallor & $6(1.9)$ & $3(2.8)$ & 9 & $3(1.5)$ & 13 & NA \\
\hline Can't tell & $19(6.1)$ & $10(9.2)$ & 3 & $9(4.4)$ & 9 & 0.152 \\
\hline Other & $19(6.1)$ & $8(7.3)$ & 4 & $11(5.4)$ & 8 & 0.661 \\
\hline
\end{tabular}

\subsubsection{Indicators for recognising non-crisis problems}

All respondents reported at least one indicator for recognising non-crisis problems on days they did not consider their problems as crisis (Table 31). Both groups ranked changes in urine colour ( $n=238)$, jaundice $(n=203)$ and fever (219) as the first three most common indicators, and swollen joint $(\mathrm{n}=104)$ as the sixth indicator. Both groups were equally likely to experience all signs and symptoms except dizziness and collapse; adults were more than twice as likely to report this than children $\left(\chi^{2}=13.182, \mathrm{df}=1, \mathrm{p}<.001\right)$.

TABLE 31. INDICATORS FOR RECOGNISING NON-CRISIS PROBLEMS

\begin{tabular}{|l|r|r|r|r|r|r|}
\hline Symptoms & \multicolumn{1}{|c|}{$\begin{array}{c}\text { Total } \\
\mathbf{n}(\boldsymbol{\%})\end{array}$} & $\begin{array}{c}\text { Adults } \\
\mathbf{n}(\boldsymbol{\%})\end{array}$ & Rank & $\begin{array}{c}\text { Children } \\
\mathbf{n}(\boldsymbol{\%})\end{array}$ & Rank & p \\
\hline Urine colour changes & $238(76.0)$ & $84(77.1)$ & 1 & $154(75.5)$ & 1 & 0.864 \\
\hline Yellow eyes or body & $203(64.9)$ & $73(67.0)$ & 2 & $130(63.7)$ & 3 & 0.653 \\
\hline High hotness of body & $219(70.0)$ & $69(63.3)$ & 3 & $150(73.5)$ & 2 & 0.080 \\
\hline Paleness of eyes & $145(46.3)$ & $55(50.5)$ & 4 & $90(44.1)$ & 5 & 0.341 \\
\hline Prolong crying * & $114(36.4)$ & $6(5.5)$ & 12 & $108(52.9)$ & 4 & $\mathrm{NA}$ \\
\hline Abdominal pain & $109(34.8)$ & $45(41.3)$ & 5 & $64(31.4)$ & 7 & 0.103 \\
\hline Swollen joint & $104(33.2)$ & $39(35.8)$ & 6 & $65(31.9)$ & 6 & 0.565 \\
\hline Difficulty in breathing & $81(25.9)$ & $27(24.8)$ & 7 & $54(26.5)$ & 8 & 0.848 \\
\hline Vomiting & $57(18.2)$ & $17(15.6)$ & 9 & $40(19.6)$ & 10 & 0.470 \\
\hline Diarrhoea & $52(16.6)$ & $16(14.7)$ & 10 & $36(17.6)$ & 11 & 0.608 \\
\hline Swollen hands and feet* & $51(16.3)$ & $0(0.0)$ & 13 & $51(25.0)$ & 9 & $\mathrm{NA}$ \\
\hline Dizziness or collapse & $26(8.3)$ & $18(16.5)$ & 8 & $8(3.9)$ & 12 & $<\mathbf{0 . 0 0 1}$ \\
\hline Painful erection* $(\mathrm{n}=145)$ & $13(9.0)$ & $7(16.7)$ & 11 & $6(5.6)$ & 13 & 0.054 \\
\hline
\end{tabular}

*Only related to children. $* *$ Only applied to male participants 


\subsubsection{Self Treatment}

Table 32 presents the SCD problems which respondents self-treated. Most responents reported treating pain, fever and jaundice management and very few treated priapism.

TABLE 32. SUMMARY OF SCD PROBLEM REQUIRING SELF TREATMENT ACTION

\begin{tabular}{|l|r|r|r|r|r|}
\hline Action & \multicolumn{1}{|c|}{$\begin{array}{c}\text { Total } \\
\mathbf{n}(\boldsymbol{\%})\end{array}$} & $\begin{array}{c}\text { Adults } \\
\mathbf{n}(\boldsymbol{\%})\end{array}$ & \multicolumn{1}{c|}{ Rank } & \multicolumn{1}{c|}{$\begin{array}{c}\text { Children } \\
\mathbf{n}(\boldsymbol{\%})\end{array}$} & \multicolumn{1}{|c|}{ Rank } \\
\hline Pain & $249(78.8)$ & $100(31.9)$ & 1 & $146(68.1)$ & 1 \\
\hline Fever & $166(53.0)$ & $45(27.1)$ & 3 & $121(72.9)$ & 2 \\
\hline Jaundiced & $177(56.5)$ & $81(45.8)$ & 2 & $96(54.2)$ & 3 \\
\hline Severe headache & $111(35.5)$ & $42(37.8)$ & 5 & $69(62.2)$ & 4 \\
\hline Anaemia & $103(32.9)$ & $44(42.7)$ & 4 & $59(57.3)$ & 5 \\
\hline Hip problems & $43(13.7)$ & $33(76.7)$ & 6 & $10(23.3)$ & 8 \\
\hline Severe bodily weakness & $42(13.4)$ & $20(47.6)$ & 7 & $22(52.4)$ & 7 \\
\hline Abdominal swelling & $41(13.1)$ & $16(39.0)$ & 8 & $25(61)$ & 6 \\
\hline Leg ulcers & $12(3.8)$ & $5(41.7)$ & 9 & $7(58.3)$ & 9 \\
\hline Priapism & $8(5.5)$ & $5(62.5)$ & 9 & $3(37.5)$ & 10 \\
\hline
\end{tabular}

9.3.4.1 Treating painful crisis. Table 33 presents the summary of responses that were recorded for pain management. A total of 249 responses indicated 12 main strategies used to manage pain at home. Pain medication was the most reported action by one fifth of both groups. Approximately one tenth of the responses also indicated use of other unspecified medications. Another tenth $(10.2 \%)$ of the responses concerned use of multivitamins and penicillin $\mathrm{V}$ to manage pain and a small percentage (2.9\%) concerned application of cold compress whilst bandaging was exclusively reported by a small proportion of children only (1.0\% overall). The two groups showed significant differences on the reported use of five strategies. The adult group were more likely to report use of unspecified medicines $(\chi 2$ $=8.299, \mathrm{df}=1, \mathrm{p}=0.004)$, folic acid, and multivitamins $(\chi 2=8.996, \mathrm{df}=1, \mathrm{p}=0.003)$, whilst the children group recorded significantly greater use of local topical creams $\left(\chi^{2}=6.822, \mathrm{df}=1\right.$, $\mathrm{p}=.009)$. The local topical creams commonly mentioned were shea butter, maame dagomba, ultimate cream, harsh cream and dompe-dompe. 
TABLE 33. SELF-TREATMENT ACTIONS FOR PAINFUL EPISODES

\begin{tabular}{|l|r|r|r|r|r|r|}
\hline Condition & \multicolumn{1}{|c|}{$\begin{array}{c}\text { Total } \\
\mathbf{n}(\boldsymbol{\%})\end{array}$} & $\begin{array}{c}\text { Adults } \\
\mathbf{n}(\boldsymbol{\%})\end{array}$ & Rank & \multicolumn{1}{c|}{$\begin{array}{c}\text { Children } \\
\mathbf{n}(\boldsymbol{\%})\end{array}$} & Rank & p \\
\hline Pain medication & $69(22.0)$ & $31(28.04)$ & 1 & $38(18.6)$ & 1 & .064 \\
\hline $\begin{array}{l}\text { Un-specified } \\
\text { medication }\end{array}$ & $32(10.2)$ & $19(17.4)$ & 2 & $13(6.4)$ & 5 & $\mathbf{. 0 0 4}$ \\
\hline Hot compress & $30(9.6)$ & $9(8.3)$ & 4 & $21(10.3)$ & 3 & .703 \\
\hline Folic acid or Multivite & $27(8.6)$ & $17(15.6)$ & 3 & $10(4.9)$ & 7 & $\mathbf{. 0 0 3}$ \\
\hline Topical cream local & $24(7.7)$ & $8(1.8)$ & 5 & $22(10.8)$ & 2 & $\mathbf{. 0 0 9}$ \\
\hline $\begin{array}{l}\text { Topical cream } \\
\text { conventional }\end{array}$ & $20(6.4)$ & $7(6.4)$ & 6 & $13(6.4)$ & 5 & NA \\
\hline Previous medication & $15(4.8)$ & $0(0.0)$ & 11 & $15(7.4)$ & 4 & NA \\
\hline Penicillin V & $5(1.6)$ & $1(0.9)$ & 10 & $4(2.0)$ & 9 & NA \\
\hline Cold compress & $9(2.9)$ & $3(2.8)$ & 9 & $6(2.9)$ & 8 & NA \\
\hline Hydration & $5(1.6)$ & $5(4.6)$ & 8 & $0(0.0)$ & 12 & NA \\
\hline Bandaging & $3(1.0)$ & $0(0.0)$ & 11 & $3(1.5)$ & 11 & NA \\
\hline Other & $10(3.2)$ & $6(5.5)$ & 7 & $4(2.0)$ & 9 & NA \\
\hline
\end{tabular}

9.3.4.2 Managing fever. A total of 166 responses were obtained in relation to six ways of managing fever of which a quarter use antipyretic medication (Table 34). Approximately one in twenty, mostly children, reported unspecified hospital medicine (s) and 6.7\%, slightly more of the adults used antimalarial, penicillin $\mathrm{V}$ or over-the-counter medication. The children's group tended to be regular users of antipyretic medication $(\chi 2=7.820, \mathrm{df}=1$, $\mathrm{p}=.005)$ and cold bath/tepid sponging $(\chi 2=8.394, \mathrm{df}=1, \mathrm{p}=.001)$ significantly more than the adults. The "other" related to use of multivitamin for children, and among adults these involved herbal medicines, avoiding oily foods, use of folic acid, Zincovite and penicillin V.

9.3.4.3 Managing jaundice. A total of 177 responses reported nine strategies for managing jaundice (Table 34). The most common was use of oral fluids with 75 responses $(24.0 \%)$. Respondents also reported using various forms of anti-malarial and pain medicines $(8.6 \%)$ to manage their jaundice. A small proportion (3.2) reported doing nothing to manage their jaundice. Adults tended to report use of oral hydration $(\chi 2=10.008, \mathrm{df}=1, \mathrm{p}=0.002$ and blood tonic $\left(\chi^{2}=5.710, \mathrm{df}=1, \mathrm{p}=0.010\right)$ significantly more than children. 
TABLE 34. SELF-TREATMENT ACTION FOR FEVER, JAUNDICE, AND ANAEMIA

\begin{tabular}{|l|r|r|r|r|r|r|}
\hline Condition & $\begin{array}{r}\text { Total } \\
\mathbf{n}(\boldsymbol{\%})\end{array}$ & $\begin{array}{c}\text { Adults } \\
\mathbf{n}(\boldsymbol{\%})\end{array}$ & Rank & $\begin{array}{r}\text { Children } \\
\mathbf{n}(\boldsymbol{\%})\end{array}$ & Rank & $\mathbf{p}$ \\
\hline Fever & & & & & & \\
\hline Antipyretics & $83(26.5)$ & $18(16.5)$ & 1 & $65(31.9)$ & 1 & $\mathbf{0 . 0 0 5}$ \\
\hline Cold bath/tepid sponging & $31(9.9)$ & $3(2.8)$ & 5 & $28(13.7)$ & 2 & $\mathbf{0 . 0 0 1}$ \\
\hline Antimalaria/OCT/Pen V & $21(6.7)$ & $9(8.3)$ & 2 & $12(5.9)$ & 3 & 0.574 \\
\hline Hospital med & $14(4.5)$ & $2(1.8)$ & 6 & $12(5.9)$ & 3 & NA \\
\hline Hydration & $4(1.3)$ & $4(3.7)$ & 4 & $0(0.0)$ & 7 & NA \\
\hline Rubbing or massaging & $3(1.0)$ & $0(0.0)$ & 7 & $3(1.5)$ & 5 & NA \\
\hline Other & $10(3.2)$ & $9(8.3)$ & 2 & $1(0.5)$ & 6 & 0.001 \\
\hline Jaundice & & & & & & \\
\hline More water & $75(24.0)$ & $38(34.9)$ & 1 & $37(18.1)$ & 1 & $\mathbf{0 . 0 0 2}$ \\
\hline Hospital/normal diet & $37(11.8)$ & $13(11.9)$ & 2 & $24(11.8)$ & 2 & 1.00 \\
\hline Malaria medicines & $20(6.4)$ & $11(10.1)$ & 3 & $9(4.4)$ & 3 & 0.086 \\
\hline Nothing & $11(3.5)$ & $4(3.7)$ & 5 & $7(3.4)$ & 4 & 1.000 \\
\hline Special diet & $9(2.9)$ & $3(2.8)$ & 6 & $6(2.9)$ & 5 & 1.000 \\
\hline Pain medicine & $7(2.2)$ & $3(2.8)$ & 6 & $4(2.0)$ & 6 & NA \\
\hline OCT* & $4(1.3)$ & $0(2.0)$ & 9 & $4(2.0)$ & 6 & NA \\
\hline Blood tonic & $9(2.9)$ & $7(6.4)$ & 4 & $2(1.0)$ & 9 & $\mathbf{0 . 0 1 0}$ \\
\hline Other & $5(1.6)$ & $2(1.8)$ & 8 & $3(1.5)$ & 8 & NA \\
\hline Anaemia & & & & & & \\
\hline Blood tonic conventional & $29(9.3)$ & $8(7.3)$ & 1 & $21(10.3)$ & 1 & 0.513 \\
\hline Special diet & $20(6.4)$ & $7(6.4)$ & 2 & $13(6.4)$ & 2 & NA \\
\hline Home prepared tonic & $16(5.1)$ & $7(6.4)$ & 2 & $9(4.4)$ & 3 & 0.617 \\
\hline Nothing & $12(3.8)$ & $7(6.4)$ & 2 & $5(2.5)$ & 4 & 0.119 \\
\hline Hospital/usual medicine & $8(2.6)$ & $4(3.7)$ & 6 & $4(2.0)$ & 6 & 0.591 \\
\hline Traditional medicine & $9(2.9)$ & $7(6.4)$ & 2 & $2(1.0)$ & 7 & $\mathbf{0 . 0 1 0}$ \\
\hline Other & $9(2.9)$ & $4(3.7)$ & 6 & $5(2.5)$ & 4 & 0.724 \\
\hline Note.
\end{tabular}

Note. The respondents reporting doing nothing said their jaundice or jaundice normally resolve or spontaneously or didn't know what to do

Table 35 reports treatment actions for anaemia, headaches, weakness, ulcers and abdominal swelling.

9.3.4.4 Anaemia management. A total of 103 responses were recorded regarding managing anaemia with six key strategies (Table 35). Conventional blood tonics were the most frequent overall (9.2\%) and Zincovit was the most mentioned among both groups.

Others were Madam Catherine and Feroglobin. Few respondents prepared their own home tonics using blended kwawunsusua (Turkey berry). Other combinations mentioned were: kwawunsusua with milk, egg yolk with milk, kwawunsusua with honey, and bedru (green leaves). The special diet mentioned by $(6.4 \%)$ the respondents usually contained kontomire 
(cocoyam leaves) in combination with other nutrients such as soya beans and shrimps. A small proportion $(3.8 \%)$ did nothing in managing their anaemia.

TABLE 35. SELF-TREATMENT ACTION FOR HEADACHES, WEAKNESS, ULCERS AND ABDOMINAL SWELLING

\begin{tabular}{|c|c|c|c|c|c|c|}
\hline Condition & $\begin{array}{l}\text { Total } \\
\text { n }(\%)\end{array}$ & $\begin{array}{l}\text { Adults } \\
\text { n }(\%)\end{array}$ & Rank & $\begin{array}{c}\text { Children } \\
\text { n }(\%) \\
\end{array}$ & Rank & $\mathbf{p}$ \\
\hline \multicolumn{7}{|l|}{ Headache } \\
\hline Pain killer only & $82(26.2)$ & 29 (26.6) & 1 & $53(26.0)$ & 1 & 0.110 \\
\hline Pain killer \& topical & $6(1.9)$ & $2(1.8)$ & 3 & $4(2.0)$ & 3 & NA \\
\hline $\begin{array}{l}\text { Pain killer \& other } \\
\text { medicines }\end{array}$ & $5(2.5)$ & $0(0.0)$ & 6 & $5(2.5)$ & 2 & NA \\
\hline Pain killer \& Multivite & $4(1.3)$ & $2(1.8)$ & 3 & $2(1.0)$ & 4 & NA \\
\hline Nasal or eye drops & $3(1.0)$ & $1(0.9)$ & 5 & $2(1.0)$ & 4 & NA \\
\hline $\begin{array}{l}\text { Pain killer \& Herbal } \\
\text { medicines }\end{array}$ & $1(1.0)$ & $0(0.0)$ & 6 & $1(0.5)$ & 7 & NA \\
\hline Other & $9(2.9)$ & $7(6.4)$ & 2 & $2(1.0)$ & 4 & NA \\
\hline \multicolumn{7}{|l|}{ Sudden weakness } \\
\hline Gave medicines & $20(6.4)$ & $11(10.1)$ & 1 & $9(4.4)$ & 1 & NA \\
\hline Massage/ ointments & $9(2.9)$ & $5(4.6)$ & 2 & $4(2.0)$ & 3 & NA \\
\hline Cold or warm bath & $4(1.3)$ & $0(0.0)$ & 4 & $4(2.0)$ & 3 & NA \\
\hline Other & $9(2.9)$ & $4(3.7)$ & 3 & $5(2.5)$ & 2 & NA \\
\hline \multicolumn{7}{|l|}{ Leg ulcer } \\
\hline Wound dressing & $4(1.6)$ & $3(2.8)$ & 1 & $1(2.0)$ & 2 & NA \\
\hline Applied substance & $7(2.2)$ & $2(1.8)$ & 2 & $5(2.5)$ & 1 & NA \\
\hline \multicolumn{7}{|l|}{ Priapism } \\
\hline $\begin{array}{l}\text { Application of cold or } \\
\text { hot water }\end{array}$ & $4(1.3)$ & $3(2.8)$ & 1 & $1(0.5)$ & 1 & NA \\
\hline Oral fluids & $2(0.6)$ & $1(0.9)$ & 2 & $1(0.5)$ & 1 & NA \\
\hline Use of medicines & $2(0.6)$ & $1(0.9)$ & 2 & $1(0.5)$ & 1 & NA \\
\hline \multicolumn{7}{|l|}{ Abdominal swelling } \\
\hline Use medicines & $26(8.3)$ & $7(6.4)$ & 1 & $19(9.3)$ & 1 & NA \\
\hline Oral hydration & $6(1.9)$ & $3(2.8)$ & 2 & $3(2.8)$ & 2 & NA \\
\hline $\begin{array}{l}\text { Application of } \\
\text { warmth/cream }\end{array}$ & $4(1.3)$ & $3(2.8)$ & 2 & $1(0.50$ & 3 & NA \\
\hline Sleep or rest & $5(1.6)$ & $2(2.7)$ & 4 & $1(1)$. & 3 & NA \\
\hline Use fruits or vegetable & $2(0.6)$ & $1(0.9)$ & 5 & $1(0.9)$ & 3 & NA \\
\hline \multicolumn{7}{|l|}{ Hip problems } \\
\hline Medicines & $20(6.4)$ & $13(11.9)$ & 2 & $7(3.4)$ & 1 & 0.006 \\
\hline Topical applications & $18(5.8)$ & $15(13.8)$ & 1 & $3(1.5)$ & 2 & NA \\
\hline Other & $5(1.6)$ & $5(4.6)$ & 3 & $0(1.6)$ & 3 & NA \\
\hline
\end{tabular}

9.3.4.5 Managing headache. A total of 111 responses were obtained for managing headache (Table 35). Most respondents use analgesics only or in combination with other 
medicines. There were no significant difference between the two groups regarding headache management strategies. The "other" was rest and sleep.

9.3.4.6 Treatment of sudden weakness. Few (42) self-treatment actions responses were reported for the management of sudden weakness (Table 35). The most frequent action reported was use of medicines (6.4\%) and both groups used similar medicines, namely unspecified drugs, folic acid, penicillin V, analgesics, and antimalarial drugs. The "other" actions were "tying affected part” (2), "sleeping/resting for long hours" (2), "applying hot water to the body" (2), "eating well" (1) and "ensuring intermittent standing" (1), and applying fatwikeke (local ointment) (1)

9.3.4.7 Treatment of leg ulcers. A total of 11 responses described leg ulcer management. Leg ulcers were mainly managed by dressing or applying substances to the wound. Both groups mentioned dressing the ulcer with hot water. The substances applied by the Children group were antibiotic powder (2), ekrobewo (a local ointment usually prepared from a mixture petroleum jelly and antibiotics) (2), Gentian Violet and oshekro (1). Adult participants did not specify medicines applied to wounds and only one participant described applying a bandage.

9.3.4.8 Priapism management. A total of eight responses were given for managing priapism (Table 35). The responses from the children's group were: "washing the penis with cold water" (1), "giving more water" (1), "giving water and malarial medication" (1). Adult's responses were: "taking paracetamol” (2), "bathing and after finishing it relaxes" (1), and “drinking lots of water" (2).

9.3.4.9 Abdominal swelling. A total of 41 responses were recorded relating to selftreatment for abdominal swelling (Table 35). Use of medicines was the commonest action (8.3\%). The medicines specified were folic acid, antihelminthics, flaggy (metronidazole), Multivite and Amoxyclav. Eight respondents could not name the medicines used. Medicines mentioned included analgesics, penicillin V, alludrox, folic acid but four could not specify the names of the medicines taken. Apart from medicines, participants used oral hydration, application of warmth, rest and sleep and fruits and vegetables to manage swollen abdomen. The specific responses included "giving lots of water," (5), "applying hot water on the 
abdomen" (3), "ensuring rest" (4)" giving orange or vegetable" (2), "giving water and asking child to rest" (1), “applying tummy cream”(1)

9.3.4.10 Treatment of hip problems. Table 35 indicates the use of medicines, massage and topical creams in managing hip pains. Among children, medicines included paracetamol, antimalarial, and haematinics. Among the adults, medicines used were analgesics and folic acid. More adults reported using medicines for hip problems compared to children and the difference was statistically significant $(\chi 2=7.210, \mathrm{df}=1, \mathrm{p}=0.007)$. The specific responses included "applying hot water to the part" (7), massage with medical ice cream and other creams (7), "applying topical cream on the hip" (2), "applied topical cream and giving folic acid" (1), and "applied topical cream and gave pain killers" (1). The "other" included "taking lots of water" (3), and "jumping or exercising the hips" (2).

\subsubsection{Frequency of selected self-treatment actions.}

Tables 36 illustrate the frequency at which people used 18 researcher selected items (pre-determined self-treatment actions). Table 36 shows a comparison between regular and periodic actions by the respondents. Of the 18 self-treatment actions, ten items (use pain medication, apply topical cream, apply hot compress, massage body part, use activity therapy, use traditional medicine, tie affected part, seek spiritual support, use alcohol) recorded higher values under regular use than periodic. In contrast, higher values were recorded under periodic actions than regular on seven items (take more fluids, sleep early, said prayer, reduced activities, performed wound dressing, applied weight and used Indian). A significant difference was observed between regular was observed between periodic and regular use on eleven action as indicated by the p-values. The top five regular actions in ranked order were pain medication $(n=161)$, application of topical cream $(n=114)$, application of hot compress $(n=103)$, massage of body part $(n=88)$, and activity therapy $(n=69)$. The top five periodic actions in ranked order were use of more fluids $(n=271)$, saying prayers $(n=181)$, sleeping early $(n=170)$, reducing activities $(n=141)$ and use of pain medication $(n=108)$.

Comparing the top five regular use actions between the two groups, it was observed that four actions (use of pain medication, application of topical cream and massage) were common among the top both groups (Table 37). However, apart from pain medication that was ranked first within both groups, the remaining actions were ranked differently between 
the two groups. The two groups showed significant difference only in the use of topical cream where children reported significantly more regular use than adults $(\chi 2=15.749, \mathrm{df}=1, \mathrm{p}<$ $0.001)$.

TABLE 36. FREQUENCY OF USE OF SELECTED SELF-TREATMENT ACTIONS BY REGULAR AND PERIODIC USE

\begin{tabular}{|l|r|r|r|r|r|r|}
\hline Action & n (\%) & \multicolumn{1}{c|}{$\begin{array}{c}\text { Regular } \\
\text { Use } \\
\mathbf{n}(\boldsymbol{\%})\end{array}$} & Rank & \multicolumn{1}{c|}{$\begin{array}{c}\text { Periodic } \\
\text { use } \\
\mathbf{n}(\boldsymbol{\%})\end{array}$} & Rank & $\mathbf{p}$ \\
\hline Take more fluids & $302(96.5)$ & $31(10.3 \%)$ & 10 & $271(89.7)$ & 1 & $<\mathbf{0 . 0 0 1}$ \\
\hline Use pain medication & $269(85.9)$ & $161(59.9)$ & 1 & $108(40.1)$ & 5 & $\mathbf{0 . 0 0 5}$ \\
\hline Sleep early & $238(76.0)$ & $68(28.6)$ & 6 & $170(71.4)$ & 3 & $<\mathbf{0 . 0 0 1}$ \\
\hline Said prayers & $224(71.6)$ & $43(19.2)$ & 8 & $181(80.8)$ & 2 & $<\mathbf{0 . 0 0 1}$ \\
\hline Apply topical cream & $210(67.1)$ & $114(54.3)$ & 2 & $96(45.7)$ & 6 & 0.353 \\
\hline Reduced activities & $195(62.3)$ & $54(27.7)$ & 7 & $141(72.3)$ & 4 & $<\mathbf{0 . 0 0 1}$ \\
\hline Apply hot compress & $141(45.0)$ & $103(73.0)$ & 3 & $38(27.0)$ & 7 & $<\mathbf{0 . 0 0 1}$ \\
\hline Massage body part & $117(37.4)$ & $88(75.2)$ & 4 & $29(24.8)$ & 8 & $\mathbf{0 . 0 0 1}$ \\
\hline Use activity therapy & $96(30.7)$ & $69(71.9)$ & 5 & $27(28.1)$ & 9 & $<\mathbf{0 . 0 0 1}$ \\
\hline Use traditional medicine & $54(17.3)$ & $40(74.1)$ & 9 & $14(25.9)$ & 11 & $<\mathbf{0 . 0 0 1}$ \\
\hline Tie affected part & $49(15.7)$ & $29(59.2)$ & 11 & $20(40.8)$ & 10 & 0.112 \\
\hline Seek spiritual support & $41(13.1)$ & $27(65.9)$ & 12 & $14(34.1)$ & 11 & $\mathbf{0 . 0 1 1}$ \\
\hline Apply cold compress & $16(5.1)$ & $13(81.3)$ & 13 & $3(18.8)$ & 16 & $\mathbf{0 . 0 0 5}$ \\
\hline $\begin{array}{l}\text { Performed wound } \\
\text { dressing }\end{array}$ & $15(4.8)$ & $7(46.7)$ & 14 & $8(53.3)$ & 13 & 0.655 \\
\hline Apply weight to part & $11(3.5)$ & $4(36.4)$ & 15 & $7(63.6)$ & 14 & 0.366 \\
\hline Use alcohol & $4(1.3)$ & $3(75.0)$ & 16 & $1(25.0)$ & 17 & 0.180 \\
\hline Use Indian hemp & $4(1.3)$ & & 10 & $4(1.3)$ & 15 & $\underline{\text { NA }}$ \\
\hline
\end{tabular}

Note. P-values are based on chi square-test, goodness of fit test comparing periodic and regular use among the actions 
TABLE 37. FREQUENCY OF USE SELECTED SELF-TREATMENT ACTIONS BY ADULT AND CHILD

\begin{tabular}{|c|c|c|c|c|c|c|c|c|c|c|}
\hline \multirow[t]{2}{*}{ Action } & \multirow{2}{*}{$\begin{array}{l}\text { Total } \\
\text { n (\%) } \\
\end{array}$} & \multicolumn{4}{|c|}{ Regular use (daily or weekly) } & \multicolumn{4}{|c|}{ Periodic use (monthly or as required) } & \multirow[t]{2}{*}{$\mathbf{p}$} \\
\hline & & Adults n (\%) & $\mathbf{R}$ & Children n (\%) & $\mathbf{R}$ & Adults n (\%) & $\mathbf{R}$ & Children n (\%) & $\mathbf{R}$ & \\
\hline Use Pain medication & $269(85.9)$ & $53(58.2)$ & 1 & $108(60.7)$ & 1 & $38(41.8)$ & 6 & $70(39.3)$ & 5 & .800 \\
\hline Sleep early & $238(76.0)$ & $23(27.7)$ & 6 & $45(60.7)$ & 5 & $60(77.3)$ & 2 & $130(82.3)$ & 1 & .949 \\
\hline Said prayers & $224(71.6)$ & $15(22.7)$ & 10 & $28(17.7)$ & 8 & $51(17.7)$ & 4 & $130(82.3)$ & 1 & .496 \\
\hline Reduced activities & $195(62.3)$ & $20(26.3)$ & 7 & $34(28.6)$ & 7 & $56(73.7)$ & 3 & $85(71.4)$ & 4 & .858 \\
\hline Apply hot compress & $141(45.0)$ & $50(78.1)$ & 2 & $53(68.8)$ & 3 & $14(21.9)$ & 7 & $24(31.2)$ & 7 & .295 \\
\hline Massage body part & $117(37.4)$ & $36(76.6)$ & 3 & $52(74.3)$ & 4 & $11(23.4)$ & 10 & $18(25.7)$ & 8 & .94 \\
\hline Use activity therapy & $96(30.7)$ & $29(67.4)$ & 4 & $40(75.5)$ & 6 & $14(32.6)$ & 7 & $13(24.5)$ & 9 & .521 \\
\hline $\begin{array}{l}\text { Use traditional } \\
\text { medicine }\end{array}$ & $54(17.3)$ & $18(75.0)$ & 8 & $22(73.3)$ & 9 & $6(25.0)$ & 11 & $8(26.7)$ & 10 & 1.000 \\
\hline Tie affected part & 49 (15.7) & $18(56.3)$ & 8 & $11(64.7)$ & 12 & $14(43.8)$ & 7 & $6(35.3)$ & 12 & .789 \\
\hline Apply cold compress & $16(5.1)$ & $3(50.0)$ & 14 & $10(100.0)$ & 13 & $3(50)$ & 15 & $0(0.0)$ & 16 & .069 \\
\hline $\begin{array}{l}\text { Performed Wound } \\
\text { dressing }\end{array}$ & $15(4.8)$ & $4(44.4)$ & 13 & $3(50.0)$ & 14 & $5(55.6)$ & 13 & $3(50.0)$ & 13 & 1.000 \\
\hline Apply weight to part & $11(3.5)$ & $3(37.5)$ & 14 & $1(33.3)$ & 15 & $5(62.5)$ & 13 & $2(66.2)$ & 14 & 1.000 \\
\hline Use alcohol & $4(1.3)$ & $2(66.7)$ & 16 & $1(100.0)$ & 15 & $1(33.3)$ & 17 & $0(0.0)$ & 16 & 1.000 \\
\hline Use Indian hemp & $4(1.3)$ & $0(0.0)$ & 17 & $0(0.0)$ & 17 & $3(100.0)$ & 15 & $1(100.0)$ & 15 & NA \\
\hline
\end{tabular}

Note. P-values are based on chi-square test of independence for association between adult/ child and periodic/regular use 


\subsection{Prevalence of Conventional and Traditional Medicines Used}

Results of commonly used medicines by the participants showed that the majority of respondents $266(85.0 \%)$ used conventional medicines and $47(15 \%)$ were traditional medicines users. Most adults 99 (90.9\%) and children 187 (81.9\%) reported using conventional medicines for self-treatment. Twenty adults (18.3\%) and 27 (13.2\%) children reported use of traditional medicines. Five types of conventional medicines were commonly reported including analgesics, haematinics, topical creams, antibiotics, and antimalarial. Half of the respondents used analgesics $(50.2 \%)$ and haematinics $(49.5 \%)$ whilst one third used antibiotics (32.6\%). Adults were significantly more likely to use analgesics, antimalarial medicines, and topical creams than the children as indicated by p-values.

TABLE 38. USE OF CONVENTIONAL MEDICINES

\begin{tabular}{|l|r|r|r|r|r|r|}
\hline & $\begin{array}{c}\text { Total } \\
\text { n (\%) }\end{array}$ & $\begin{array}{c}\text { Adults } \\
\mathbf{n}(\boldsymbol{\%})\end{array}$ & Rank & $\begin{array}{c}\text { Children } \\
\mathbf{n}(\boldsymbol{\%})\end{array}$ & Rank & \multicolumn{1}{|c|}{ P } \\
\hline Analgesics & $157(50.2)$ & $75(68.8)$ & 1 & $82(40.2)$ & 2 & $<\mathbf{0 . 0 0 1}$ \\
\hline Haematinic & $155(49.5)$ & $62(56.9)$ & 2 & $93(45.6)$ & 1 & 0.074 \\
\hline Antibiotic & $102(32.6)$ & $38(34.9)$ & 3 & $64(31.4)$ & 3 & 0.616 \\
\hline Anti-malaria & $37(11.8)$ & $31(28.4)$ & 4 & $6(2.9)$ & 5 & $<\mathbf{0 . 0 0 1}$ \\
\hline Topical cream & $36(11.5)$ & $25(23.1)$ & 5 & $11(5.4)$ & 4 & $<\mathbf{0 . 0 0 1}$ \\
\hline
\end{tabular}

Table 39 outlines the traditional medicines, the reason reported for taking them and route of administration. A total of 32 traditional medicine uses were described. Of these, the Adults group described 15 whilst the Children group described 15 . Twenty three of the traditional medicines described were herbal or vegetable in origin. Of these 12 were described by the Adults and 11 by the Children groups. Nine topical creams were also described for which adults accounted for 3 and children for 6 .

The major reason cited by both groups for using herbs were malaria, anaemia and pain, but pain was main reason for using topical creams. Amongst adults, the reason cited for using herbs were malaria (4), pain (3), anaemia (3), eye problems (1), headache (1), jaundice (1), to minimise disease (1), and to promote passage of blood through the veins (1). In the children group the reasons for using herbs were pain (4), malaria (1), whole disease (1), fever (1), and spiritual protection (1). 
All adult respondents used topical cream for pain (3), but in the case of children, the reasons were pain (4), to cure disease (1) and spiritual protection (1). All herbal and vegetable medicines were administered orally except one (dua kaako + nyamedua) that which were administered through the nose. All topical creams were applied on the skin.

TABLE 39. USE OF TRADITIONAL MEDICINE

\begin{tabular}{|c|c|c|}
\hline Traditional Medicine $(\mathrm{n}=32)$ & Reason for use & Route \\
\hline \multicolumn{3}{|l|}{ Adult } \\
\hline \multicolumn{3}{|l|}{ Herbs and vegetables } \\
\hline Pawpaw leaves + Gyama leaves & Pain & Swallowed \\
\hline Fawohoto awuarade & Pain & Swallowed \\
\hline Boiled Tick + Nim tree + grass & Jaundice and pain & Swallowed \\
\hline Angel herbal mixture & Malaria & Swallowed \\
\hline Taabia bitters & Malaria & Swallowed \\
\hline Bonhagu waakyi & Malaria & \\
\hline $\begin{array}{l}\text { Greengrass }+ \text { nim tree }+ \text { pinapple peals }+ \text { pearl } \\
\text { leaves }\end{array}$ & Anaemia and malaria & Swallowed \\
\hline Kwaunsusua & Anaemia & Swallowed \\
\hline Kwaunsusua + milk + cokecacoa + tomatoe past & Anaemia & Swallowed \\
\hline Dua kaako + Nyamedua & $\begin{array}{l}\text { Headache, minimise } \\
\text { whole disease }\end{array}$ & $\begin{array}{l}\text { Applied through nose } \\
\text { Swallowed }\end{array}$ \\
\hline Boiled pawpaw leaves + Mahogany leaves & $\begin{array}{l}\text { To promote passage of } \\
\text { blood through the veins }\end{array}$ & Swallowed \\
\hline Boiled Tick + some herbs & Eye problems & Swallowed \\
\hline \multicolumn{3}{|l|}{ Topical creams } \\
\hline Ice cool cream & Pain & Topical application \\
\hline Medical ice cream & Pain & Topical application \\
\hline Pain gell & Pain & Topical application \\
\hline \multicolumn{3}{|l|}{ Children } \\
\hline \multicolumn{3}{|l|}{ Herbs and vegetables } \\
\hline Yellow fever & Jaundice & Swallowed \\
\hline Kwawunsusua & Anaemia & Eaten \\
\hline Unknown herbs & $\begin{array}{l}\text { To manage whole } \\
\text { disease }\end{array}$ & Swallowed \\
\hline $\begin{array}{l}\text { Leaves of kwawunsusua mixed with honey and } \\
\text { milk }\end{array}$ & Anaemia & Swallowed \\
\hline Kwawunsua with milk & Anaemia & Eaten \\
\hline Mixture of lemon grass, black pepper, & Fever & $\begin{array}{l}\text { Swallowed and grinded some to } \\
\text { applied on affected }\end{array}$ \\
\hline Herbs but can't recall & Pain & Topical application \\
\hline Herbs can't remember (2) & Anaemia & $\begin{array}{l}\text { Swallowed } 1, \text { mixed milk and } \\
\text { drank to treat shortage of blood }\end{array}$ \\
\hline Owhem herbal mixture & Pain & Swallowed \\
\hline Some herbs picked & Malaria & Swallowed \\
\hline I take bedru & Anaemia & Eaten \\
\hline \multicolumn{3}{|l|}{ Topical cream } \\
\hline Ice cool cream & Pain & Topical application \\
\hline Maame dagomba (cream) & Pain & Topical application \\
\hline Maame dagomba & Pain & Topical application \\
\hline Kojokoo cream & Pain & Topical application \\
\hline Shear butter & To cure disease & Topical application \\
\hline MOPAoil** & For spiritual protection & Topical application \\
\hline
\end{tabular}




\subsection{Evaluation of Self-Treatment Actions}

Respondents' assessment of the impacts of self-treatment treatment are presented in Table 40. The majority of respondents rated their self-management actions as helpful with frequencies of 50 to $100 \%$ that were exclusively recorded as helpful or very helpful.

TABLE 40. RESPONDENT'S EVALUATION OF SELF-TREATMENT ACTIONS FOR INDIVIDUAL CONDITIONS

\begin{tabular}{|l|r|r|r|r|r|r|r|}
\hline & & \multicolumn{1}{l|}{ Helpful } & \multicolumn{1}{l|}{ Not helpful } & \multicolumn{2}{l|}{ Neither } \\
\hline Action & $\begin{array}{l}\text { Total } \\
\mathrm{n}(\%)\end{array}$ & $\begin{array}{l}\text { Adult } \\
\mathrm{n}(\%)\end{array}$ & $\begin{array}{l}\text { Children } \\
\mathrm{n}(\%)\end{array}$ & $\begin{array}{l}\text { Adult } \\
\mathrm{n}(\%)\end{array}$ & $\begin{array}{l}\text { Children } \\
\mathrm{n}(\%)\end{array}$ & $\begin{array}{l}\text { Adults } \\
\mathrm{n}(\%)\end{array}$ & $\begin{array}{l}\text { Children } \\
\mathrm{n}(\%)\end{array}$ \\
\hline $\begin{array}{l}\text { Pain } \\
\text { management }\end{array}$ & $193(61.7)$ & $64(87.7)$ & $\begin{array}{r}110 \\
(91.7)\end{array}$ & $6(8.2)$ & $5(4.2)$ & $3(4.1)$ & $5(4.2)$ \\
\hline $\begin{array}{l}\text { Fever } \\
\text { management }\end{array}$ & $147(47.0)$ & $39(95.1)$ & $98(92.5)$ & $2(4.9)$ & $4(3.8)$ & $0(0.0)$ & $4(3.8)$ \\
\hline Headache & $131(41.9)$ & $46(88.5)$ & $77(97.5)$ & $4(7.7)$ & $1(1.3)$ & $2(3.8)$ & $1(1.3)$ \\
\hline $\begin{array}{l}\text { Sudden } \\
\text { weakness }\end{array}$ & $54(17.3)$ & $25(89.3)$ & $24(92.3)$ & $1(3.9)$ & $1(3.6)$ & $2(7.1)$ & $1(3.8)$ \\
\hline Leg ulcers & $13(4.2)$ & $7(100)$ & $4(66.7)$ & $0(0.0)$ & $2(33.3)$ & $0(0.0)$ & $0(0.0)$ \\
\hline Priapism* & $11(7.6)$ & $4(66.7)$ & $5(100)$ & $2(33.3)$ & $0(0.0)$ & $0(0.0)$ & $0(0.0)$ \\
\hline Jaundice & $140(44.7)$ & $53(93.0)$ & $81(97.6)$ & $1(1.8)$ & $1(1.2)$ & $3(5.3)$ & $1(1.2)$ \\
\hline Anaemia & $67(21.7)$ & $20(95.2)$ & $45(97.8)$ & $0(0.0)$ & $1(2.2)$ & $1(4.8)$ & $0(0.0)$ \\
\hline $\begin{array}{l}\text { Abdominal } \\
\text { swelling }\end{array}$ & $42(13.4)$ & $12(85.7)$ & $26(92.9)$ & $0(0.0)$ & $0(0.0)$ & $2(14.3)$ & $2(7.1)$ \\
\hline Hip problems & $40(12.8)$ & $29(100)$ & $8(72.7)$ & $0(0.0)$ & $3(27.3)$ & $0(0.0)$ & $0(0.0)$ \\
\hline Sudden seizures & $2(0.6)$ & $1(50)$ & $0(0.0)$ & $1(50)$ & $0(0.0)$ & $0(0.0)$ & $0(0.0)$ \\
\hline
\end{tabular}

\subsection{Health Status and Quality of Life}

Table 41 presents self-rating of health status and QOL. The majority of both groups rated health status and quality of life overall as good or better. Adults were approximately two more likely than children to rate their health status $(13.8 \%$ vs $8.8 \%)$ and QOL $(27.5 \%$ versus $17.5 \%$ ) as fair or poor. These differences were not statistically significant. A Spearman's correlation between health status and QOL and total self-management actions presented in Table 41 did not identify any significant association between health status $\left(r_{\mathrm{s}}=0.032, \mathrm{p}=\right.$ $0.574)$ and quality of life $\left(r_{\mathrm{s}}=0.034, \mathrm{p}=0.594\right)$. 
TABLE 41. SELF-RATED HEALTH STATUS AND QUALITY OF LIFE

\begin{tabular}{|l|l|r|r|r|r|}
\hline Outcome & Value & \multicolumn{1}{|c|}{$\begin{array}{l}\text { Total } \\
\mathbf{n = 3 1 3}(\boldsymbol{\%})\end{array}$} & $\begin{array}{l}\text { Adults } \\
\mathbf{n = 1 0 9}(\boldsymbol{\%})\end{array}$ & $\begin{array}{l}\text { Children } \\
\mathbf{n = 2 0 4}(\boldsymbol{\%})\end{array}$ & P \\
\hline Health status & Good or better & $280(89.5)$ & $94(86.2)$ & $186(91.2)$ & 0.245 \\
& Fair or poor & $33(10.5)$ & $15(13.8)$ & $18(8.8)$ & \\
& & & & & \\
\hline Quality of life & Good or better & $247(78.9)$ & $79(72.5)$ & $168(82.4)$ & 0.058 \\
& Fair or poor & $6(21.1)$ & $30(27.5)$ & $36(17.5)$ & \\
& & & & & \\
\hline
\end{tabular}

Note. p-values based on chi-square test for independence among adults/children

\subsection{Self-Management Resources}

Table 42 describes the medicines and logistic resources, sources of information and self rated knowledge of SCD care preported by the respondents. The majority of the respondents indicated possession of medicines (folic acid, pain medication and topical cream) with fewer posessing other self-managemnt resources. Children were significantly more likely to possess warm clothing $\left(\chi^{2}=10.039, \mathrm{df}=1, \mathrm{p}=0.001\right)$ than adults. Adults on the otherhand reported possessing diary or record book than children $\left(\chi^{2}=4.650, \mathrm{df}=1, \mathrm{p}=\right.$ 0.031). A Spearman's test for correlation between total self-management actions and possession of medicinal and logistic resources showed a week positive significant correlation $\left(r_{\mathrm{s}}=0.122, \mathrm{p}=0.031\right)$.

Mass media in the form of radio and TV constituted the major source of information for all groups with more than half of the respondents also receiving information from health professionals. In addition, $13.4 \%$ of the respondents reported access information from the internet, with adults significantly more likely to use the internet than the children $\left(\chi^{2}=30.529\right.$, $\mathrm{df}=1, \mathrm{p}<.001)$.

The majority of the respondents rated their knowledge as good or better (good, very good, and excellent). Adults (83.5\%) rated their knowledge higher (excellent, very goog, good) than the children's groups $(70.4 \%)$, this was statistically significant $\left(\chi^{2}=11.686, \mathrm{df}=4\right.$, $p=0.020)$. A Spearman's test for correlation between total self-management actions and respondents self-rated knowledge revealed a very weak insignificant positive correlation $\left(r_{s}=\right.$ $0.031, \mathrm{p}=0.581)$. 
TABLE 42. SELF-MANAGEMENT RESOURCES

\begin{tabular}{|c|c|c|c|c|c|c|}
\hline Activity & $\begin{array}{c}\text { Total } \\
\mathbf{n}=\mathbf{3 1 3}\end{array}$ & $\begin{array}{l}\text { Adults } \\
\mathrm{n}=109\end{array}$ & Rank & $\begin{array}{c}\text { Children } \\
n=204\end{array}$ & Rank & $\mathbf{p}$ \\
\hline \multicolumn{7}{|c|}{ Medicines and material resources } \\
\hline Folic acid & $306(97.8)$ & $108(99.1)$ & 1 & $198(97.1)$ & 1 & 0.566 \\
\hline Warm clothing & $267(85.3)$ & $83(76.1)$ & 4 & $184(90.2)$ & 2 & 0.001 \\
\hline Pain medication & $265(84.7)$ & $92(84.4)$ & 2 & $173(84.8)$ & 3 & 1.000 \\
\hline Topical cream & $231(73.8)$ & $84(77.1)$ & 3 & $147(72.1)$ & 4 & 0.410 \\
\hline Hot water bottle & $57(18.2)$ & $18(16.5)$ & 5 & $39(19.1)$ & 5 & 0.678 \\
\hline $\begin{array}{l}\text { Education } \\
\text { materials }\end{array}$ & $39(12.5)$ & $18(16.5)$ & 5 & $21(10.3)$ & 6 & 0.159 \\
\hline Wound dressing & $27(8.6)$ & $14(12.8)$ & 7 & $13(6.4)$ & 7 & 0.083 \\
\hline Thermometer & $21(6.9)$ & $9(8.3)$ & 9 & $12(5.9)$ & 8 & 0.574 \\
\hline $\begin{array}{l}\text { Diary or record } \\
\text { book }\end{array}$ & $18(5.8)$ & $11(10.1)$ & 8 & $7(3.4)$ & 9 & $\mathbf{0 . 0 3 1}$ \\
\hline \multicolumn{7}{|c|}{ Sources of information for SCD care } \\
\hline Radio & $204(65.2)$ & $77(70.6)$ & 1 & $127(62.3)$ & 1 & 0.174 \\
\hline Television & $189(60.4)$ & $66(60.6)$ & 2 & $123(60.3)$ & 2 & 1.000 \\
\hline Health worker & $171(54.6)$ & $51(46.8)$ & 3 & $120(58.8)$ & 3 & 0.055 \\
\hline Relatives & $90(28.8)$ & $39(35.8)$ & 4 & $51(25.0)$ & 4 & 0.610 \\
\hline Internet & $42(13.4)$ & $31(28.4)$ & 5 & $11(5.4)$ & 6 & $<0.001$ \\
\hline Friends & $60(19.2)$ & $27(24.8)$ & 6 & $33(16.2)$ & 5 & 0.091 \\
\hline \multicolumn{7}{|c|}{ Self rated knowledge of SCD care } \\
\hline Excellence & $66(21.1)$ & $20(18.3)$ & 3 & $46(22.5)$ & 2 & \multirow[t]{5}{*}{0.020} \\
\hline Very good & $101(32.3)$ & $39(35.8)$ & 1 & $62(30.4)$ & 1 & \\
\hline Good & $68(21.7)$ & $32(29.4)$ & 2 & $36(17.6)$ & 4 & \\
\hline Fair & $61(19.5)$ & $16(14.7)$ & 4 & $45(22.1)$ & 3 & \\
\hline Poor & $17(5.4)$ & $2(1.8)$ & 5 & $15(7.4)$ & 5 & \\
\hline
\end{tabular}

Note. Self rated knowledge totals up to $100 \%$

\subsection{Challenges in Caring for SCD}

Table 43 shows the results of common challenges respondents reported to have encountered in managing their or children's SCD. Financial constraints rank first for both adults and children. The remaining were challenges rank differently between groups with the majority of the concerns related to direct care of disease including cost of treatment, care related stress, disease symptomatology, nutrition and feeding challenges, attending hospital, time wasting, distance from home to hospital. Caring for CSD was not considered a problem by $15.4 \%$ of respondents. 
TABLE 43. CHALLENGES ASSOCIATED WITH CARING FOR SCD

\begin{tabular}{|l|r|r|r|r|r|}
\hline Challenge & \multicolumn{1}{|c|}{$\begin{array}{c}\text { Total } \\
\mathbf{n}(\boldsymbol{\%})\end{array}$} & $\begin{array}{c}\text { Adults } \\
\mathbf{n}(\boldsymbol{\%})\end{array}$ & Rank & \multicolumn{1}{c|}{$\begin{array}{c}\text { Children } \\
\mathbf{n}(\boldsymbol{\%})\end{array}$} & Rank \\
\hline Financial constraints & $42(17.9)$ & $17(20.7)$ & 1 & $25(16.4)$ & 1 \\
\hline Cost of treatment & $27(11.5)$ & $11(13.4)$ & 3 & $16(10.5)$ & 4 \\
\hline Care related stress & $23(9.8)$ & $6(7.3)$ & 6 & $17(11.2)$ & 3 \\
\hline Stubbornness/tantrum & $17(7.3)$ & $2(2.4)$ & 9 & $15(9.9)$ & 5 \\
\hline Disease symptomatology & $14(6.0)$ & $11(13.4)$ & 3 & $3(2.0)$ & 12 \\
\hline $\begin{array}{l}\text { Nutrition/feeding } \\
\text { challenges }\end{array}$ & $12(5.1)$ & $0(0.0)$ & 12 & $12(7.9)$ & 6 \\
\hline Time wasting & $10(4.3)$ & $5(6.1)$ & 7 & $5(3.3)$ & 9 \\
\hline Attending hospital & $7(3.0)$ & $3(3.7)$ & 8 & $4(2.6)$ & 10 \\
\hline Stigmatisation & $6(2.6)$ & $2(2.4)$ & 9 & $4(2.6)$ & 10 \\
\hline Interference with work & $8(3.4)$ & $1(1.2)$ & 11 & $7(4.6)$ & 7 \\
\hline Other* & $14(6.0)$ & $7(8.5)$ & 5 & $7(4.6)$ & 7 \\
\hline Not a problem & $36(15.4)$ & $13(15.9)$ & 2 & $23(15.1)$ & 2 \\
\hline
\end{tabular}

Note. Table not suitable for chi-square test for independence between child/adultas $52.8 \%$ of cells had expected counts less than 5 .

* Having more children, lack of male spouce support, long distance from home to hospital/clinic.

This chapter presented the results of the quantitative survey among patients and parents. The subsequent chapter will focus on discussion of the results of all the four stages of data collection and analysis. 


\section{Chapter 10: Discussion and Conclusion}

\subsection{Introduction}

This four stage mixed method study was undertaken to understand self-management strategies for SCD in Ghana. The objectives of the study were to:

- Establish self-management advice provided in SCD focused websites and by SCD experts in Ghana;

- Identify the measures patients and families take to prevent illness and reduce complications;

- Determine the strategies patients and families use in monitoring their disease;

- Determine the strategies patients and families use to recognise SCD related problems and complications;

- Determine the specific self-management actions taken by patients in response to recognised problems and complications;

- Elicit the major factors underpinning self-management practices among patients and families.

As no SCD self-management framework was located in literature that was suitable for the Ghanaian context, the study commenced with a review of SCD-focused websites, followed by interviews with Ghanaian health professionals with experience in caring for people with SCD. These two stages gathered the advice provided to patients for their day-to-day self-care and the constructs, concepts and variables central to SCD self-management. Stage 3 involved interviews of adult SCD patients and parents of children with SCD regarding their selfmanagement practices. The results of the three qualitative stages and the literature review informed the development of a questionnaire, which was administered to a cross-sectional sample of adult patients with SCD and parents of children with SCD.

The findings are described in detail in the previous four chapters. This chapter discusses and draws attention to key findings only. The discussion is presented in seven sections, the first of which covers the relevance and representativeness of the samples. Secondly, I summarise the self-management recommendations from the websites and the health professionals, thirdly I summarise the patients' and parents' practices from their interviews and survey and fourthly I discuss the practices adopted under the headings 'safe and effective practices' and 'unsafe practices'. Fifthly, I discuss the findings in relation to self-management 
theories. The sixth section covers the limitations of the research, the seventh presents the recommendations and the concluding section presents an overview the key contributions of the research. Of note, the use of the word participant refers to the health professionals and adults and parents interviewed, and the word respondent refers to survey results.

\subsection{Sample Characteristics}

Four samples were involved in this study. The first sample comprised 14 websites that had a primary focus on SCD patient education and support that were analysed. The second sample was the nine Ghanaian health professionals with experience in SCD management who were interviewed. The third sample was the 10 adult patients and nine parents of children with SCD who participated in interviews. The fourth sample were the 313 people who completed the survey.

The website sample included all English language sites that met the inclusion criteria of providing SCD patient focused education. As such, it is a total sample, however, only one of the websites was developed from an African context. Many of the websites included information that assumed the presence of a 24 hour, 7 day a week health services where there is easy access to emergency care and specialised SCD services. Ghana has hospital-based emergency services that can be accessed, however the SCD specific services are sparse and mainly cater for children.

The health professional sample is representative of Ghanaian experts in SCD. Five of the professionals had worked on the NSSCD Project as clinicians, educators or counsellors. The remaining four professional had attended SCD workshops and were currently working as clinicians at SCD clinics. Given this background, the recommendations of the health professionals can be assumed as reflecting current practice.

The purposefully recruited adult patients and parents of SCD children who participated in the interviews were selected to establish how they practised and understood self-management. They were not selected for representativeness. Participants described their self-management of HbSS and HbSC genotypes, which reflects the Ghanaian SCD profile [4].

The respondents surveyed were analysed and described in two ways. Firstly, the analysis grouped the respondents by role as parents of children with SCD and adult SCD 
patients and secondly by age of patient with SCD whose self-management care was referred to as children or adults. As there is no published data on the age profile of the SCD population in Ghana, it is not possible to determine the representativeness of this sample. Additionally, as Ashanti is only the region in Ghana that offers SCD screening in public health facilities, the results may not be generalisable to other regions in Ghana.

The finding that almost $60 \%$ of patients with SCD were under 35 years is similar to another study that surveyed SCD patients attending clinic in Ghana and Brazil [227, 228]. This age profile was expected for three reasons. Firstly, it is established that SCD patients have shorter life expectancy compared to the general population, hence one is likely to find more children and younger people with SCD than adults in the African population [229, 230]. Secondly, as only children focused SCD clinics existed at the time of data collection, the parents of children were more accessible than adults with SCD to be invited for the research. Thirdly, more parents of children than adults with SCD were recruited because the NSSC Project in the study region recommends children diagnosed with SCD routinely attend SCD clinic [4].

An important result in the parent subset was that fathers constituted $13.4 \%$ of parents who responded to the survey. Caring for a sick child in the Ghanaian culture is normally the responsibility of the mother; the father's role is mainly to provide financial support for the family [231]. This result could reflect recent calls for greater paternal participation in children's health care in Ghana [232].

The socio-economic profile of the respondents indicates the sample was below the Ghanaian average. The sample had higher unemployed rates (11.2\%) compared to the 2014 national average (5.2\%) [233]. More than 80\% received no income or less than GH C500.00 monthly. This finding indicates that the majority of respondents received less than the 2015 daily minimum wage of GH $\mathbb{C} 8.00$ and lived below the 2014 poverty line of GH $\mathbb{C} 3.60$ [234]. Furthermore, most respondents reported spending a significant proportion of their income on SCD, reducing spending available for other needs. 


\subsection{Key Self-Management Recommendations and Practices}

This section, presents the key self-management recommendations elicited from the websites and health professionals interviews, as well as the actions undertaken by the patients and parents who were interviewed or surveyed.

\subsubsection{Recommendations from Websites and Health Professionals}

Websites and health professionals provided recommendations on many topics. Strategies that were highly recommended by both sources concerned hydration, analgesia, nutrition, avoiding extremes of temperature (especially exposure to cold), safe activities, folic acid supplements, and physical assessments. Preventing and managing pain was the focus of most recommendations. Both sources, and especially websites, provided more health servicebased recommendations. Where patient directed actions were recommended, websites provided specific directions when compared to the direction advised by health professionals. The websites reported using resources such as hot water bottles and thermometers that could be used by most patients to perform self-management actions. Most health professionals reported that they provided limited self-management advice and three were unaware patients could perform aspects of self-management actions or possess self-management resources. Although most recommendations could be assessed as clinically safe, some are potentially unsafe.

Most recommendations were simple and within the capacity of individuals and families. However, there were other recommendations in websites that were potentially too technical for patients and families to follow. One example is advising administration of medicines in dosages calculated using kilogram body weight while other recommendations were vague and open to patients' own judgements such as advising people to avoid exhaustion without describing indicators of exhaustion. Other recommendations appear to have geographic and health service implications. There were variations in website content related to the websites hosted in countries with a temperate or tropical climate or a westernised or non-westernised culture. For example, regarding the management of fever, the North American sites usually advised against home intervention, recommending people to seek health service assistance, whereas the African site and the health professionals in Ghana recommended implementing home remedies, before reporting to the hospital. 
The key preventive health advice involves patients pursuing actions that are health promotive to maintain their health such as good nutrition; or ensuring avoidance, precaution, and moderation when patients manage potential triggers such as temperature and activities. Most of the recommended preventive health actions were similar from both sources. There were topics that had cultural and geographical implications such as advice from websites regarding precautions on air travel and scooter driving, the importance of pneumococcal and flu vaccinations, and the use of iron chelators. These are relevant to people living in temperate climates, however they assume a degree of wealth. In contrast, recommendations regarding prevention of worm and malarial infections are relevant to tropical countries including Ghana. Whilst both sources had similar recommendations, websites tended to recommend within a supportive framework compared with the health professionals. For example, the health professionals advise people to avoid swimming because of exposure to cold whereas the websites promote swimming in heated pools. Health professionals advise people to eat well, and would advise patients who had misconceptions regarding certain diet and food items. The websites recommend people seek nutritional support from professionals, and advised against consuming diet soda drinks or a high sodium diet. The websites provided advice on medications that patients must avoid (diuretics, nasal decongestants, aspirin, salicylates) but this topic was not mentioned by the health professionals.

The recommendations for self-monitoring provided by both sources included inspection and palpation of the body and, for children, observing behaviour and responding to complaints, such as tiredness. Anticipating potential triggers of crises were recognised by both sources. However, the health professionals' recommendations supported a greater focus on self-monitoring than recommended by the websites. Self-diagnostic indicators, for which people should seek immediate care, were listed by both sources. The websites recommended symptom-based indications (e.g., chest pain, numbness, bodily weakness), whereas the health professional advice related to sign-based indicators (e.g., fever, changes in urine colour, jaundice).

The more commonly recommended self-treatments were for pain management and typically listed analgesics and the application of warmth or topical creams. There were variations in the advice relating to individual conditions. Both sources recommended selftreatment for leg ulcers and dactylitis. In addition, websites described management of aseptic necrosis of the head of femur, jaundice, pains with menses, injury care, and bedwetting, 
whereas health professionals provided advice on fever and priapism. Although variabilities existed, the findings from both sources are important and useful for service development and clinical practice.

\subsubsection{Summary of Patients' and Parents' Practices}

The overall findings identify that self-management is practised daily by most respondents; the patients and parents engage in various strategies to manage SCD consistent with the conceptual framework for this study: preventive health and maintenance, selfmonitoring, self-diagnosis, self-treatment and self-evaluation. As SCD patients and carers manage approximately $90 \%$ of the daily tasks related to living with the condition [11], and coupled with a lack of an organised patient focused education programme in Ghana, it was anticipated that people construct their own self-management strategies that allow them to live with a LTC. The research identified that all patients and parents surveyed or interviewed performed at least one type of management action but with varying regularity.

Respondents reported they consciously engaged in preventive healthcare and selftreatment actions. The remaining actions (monitoring, diagnosis, evaluation) were usually carried out as automatic unconscious responses to changes in health status. Whilst people readily shared their preventive health and self-treatment actions, it took effort and guidance from the researcher for respondents and interview participants to discuss self-monitoring and self-diagnosis. Thus the findings may demonstrate only a limited scope of what is involved in the self-management of SCD by patients in Ghana. Parents were more frequent users of selfmanagement actions for their children than adults for themselves. Apart from being an adult or child, self-management was not significantly influenced by key vulnerabilities and contextual factors (Table 25). However, self-management actions were influenced by possession of medicines and other equipment. Generally, participants were unaware of the need to acquire self-management resources, such as equipment and skills, but they understood having knowledge about SCD was important.

Pain was the commonest reason most respondents took action, followed by fever and anaemia. The commonest preventive actions were frequent oral hydration, avoidance of temperature extremes (especially cold), avoiding exhaustive activities and exercises, eating a healthy diet, consuming folic acid supplementation and malaria/ infection prevention. For self-monitoring, the commonest actions included checking the eyes and urine for changes in 
colour, and observing for triggers of pain. Common self-diagnostic indicators were also eye and urine colour changes, fever, pain and unusual feelings. The participants and respondents reported utilising medicinal and non-medicinal actions to manage pain and other conditions including fever, jaundice, leg ulcers, headache, hip problems, abdominal distension and bodily weakness. Medicinal interventions were more common than non-medicinal interventions with respondents reporting a preference for conventional medicines. The majority had used traditional medicines, but only a few were current users as traditional medicines were viewed as ineffective.

Respondents mainly had positive views about themselves and expressed satisfaction with their self-management. They considered their health status and QOL as good. The survey findings found no significant correlation between self-management, QOL and health status. Respondents reported attending clinic appointments was important. Appointments provided routine health opportunities for respondents to receive new medicines, discuss problems and be advised on any additional strategies they could undertake. Access to appointments was however problematic.

\subsection{Discussion of Key Findings}

This section commences with a discussion of the key findings outlined above. This is followed by an examination of the actions taken in relation to patient-assessed effectiveness and clinical safety.

\subsubsection{Key Self-Management Practices}

The findings regarding self-management identified that patients and parents placed an emphasis on preventive health and maintenance actions, involving health promotion such as nutrition and hydration and avoidance behaviours such as avoiding of extremes of temperature and over activity. The participants in the previous SCD self-management related research reported similar areas of preventive health in USA, Brazil and Jamaica [17, 123, 173, $176,177,235]$. In contrast to the findings from the SCD self-management literature, selfmonitoring received little recognition in this study. In the USA, qualitative studies describe "self-awareness" or "listening to, and learning about the body" that identify trigger factors and recognise body sensations as the major strategies reported by patients $[17,123,176]$. Similarly, quantitative researchers focused on pain self-monitoring $[116,119,122,131,132$, 
139]. The difference in emphasis could be attributed to level of self-management education and support. The participants in the studies from the USA had exposure to structured SCD self-management education and support unlike their Ghanaian counterparts.

Regarding self-monitoring in the current study, an unanticipated finding was the use of diaries or record books among $54.6 \%$ of respondents (80.4\% parents, $6.4 \%$ adults [Table 9]). From my experience, diary use is an unknown practice for SCD care in Ghana and Africa. As diary use was not reported by the interview participants and only $5.6 \%$ of respondents referred to diaries in relation to their self-management resources (Table 20), the 54.6\% findings was unanticipated. It is possible that respondents have confused diaries with a child health record book - Road to Health Booklet/card given to mothers when their infant is born to monitor the development of the child until they are five years old.

The SCD self-management studies involving self-monitoring from the USA found that diaries or journaling are central for self-monitoring. These enable patients to understand the interactions of SCD on their body, triggers of illnesses, and their limits. Consequently, diaries enabled patients to control their disease and impact on their health $[123,176]$. Electronic diaries were used to assist patients monitor their pain, associated symptoms, and their responses to problems and facilitated early interventions by health professionals $[116,122$, 131, 132]. The use of diaries or journaling has been reported as a common feature for many other LTCs in more technologically advanced health systems [236]. The above USA based SCD studies revealed researchers efforts in providing pre-determined monitoring indicators and devices to facilitate SCD self-monitoring. These researchers demonstrated the positive impacts of structured monitoring. In Ghana, SCD self-monitoring was found to be predominantly based on patients' knowledge and skills, and mostly performed in response to perceived problems. These finding indicate that research is required in Ghana to understand the usefulness of diaries to assist patients self-monitoring and other confirm strategies.

The findings indicate that self-monitoring, using physical examination and measurements requiring the use of equipment such as thermometers, are limited in Ghana. For example splenic palpation, which should be a routine practice, was never mentioned by any of the participants, and only $39.9 \%$ of respondents reported conducting abdominal palpation. Secondly, despite the importance of fever in SCD, only $6.9 \%$ of respondents reported owning 
thermometers (Table 42). These results indicate that patient self-monitoring actions do not commonly involve gathering consistent, measurable evidence of changes in health status.

Health professionals and websites outlined a range of acute signs and symptoms that can be self-diagnosed for which patients should seek emergency clinical care. These included unusual pain, fever, difficulty in breathing, abdominal pains, severe headache, abdominal swelling, numbness/ weakness, fever, enlarged or tender spleen and jaundice. Patients referred to a limited number of these signs and symptoms including pain, eye and urine colour changes, fever. Of interest is that many patients had their own criteria for self-diagnosis. For example parents' reported red eyes indicates a crisis, yellow eyes indicates malaria, and brown urine indicates dehydration. Adults also reported unusual body sensations such as bitterness in the mouth and frequent yawning as indicators of an emerging painful crisis.

Contrary to professionals' and website recommendations, many patients did not associate these signs and symptoms as emergencies. Although patients acknowledge these signs and symptoms as deviation from normal they, especially the adults, would rather engage in behavioural adjustments or utilise self-treatment. Many would only seek clinical care if their self-treatment strategy did not resolve the problems or they considered the problem as unusual. Limited publications exist in the current literature regarding self-diagnosis in SCD from patients' perspectives. Self-diagnosis is often captured as symptom recognition in other chronic conditions such as heart failure, chronic obstructive pulmonary disease and cancers [237]. The signs and symptoms Jacob et al. [238] reported that prompt SCD patients to seek care for vase-occlusive pains are comparable with findings reported for crisis recognition in the current study (Table $31 \&$ 32) [238]. More recently, these researchers used a symptom checklist developed from this earlier study to investigate the signs and symptoms that prompted SCD patients to seek clinical care for painful crises [131]. The use of checklists is becoming common place in healthcare [239]. Considering use of checklists in terms of selfdiagnosis and monitoring will be important for SCD self-management in Ghana as a patient safety strategy.

An objective of this study was to identify respondents' characteristics that influence patients' self-management. These factors were explored qualitatively and quantitatively as vulnerability, contextual and self-management resources. The qualitative narratives show that these factors have positive and negative influences on self-care. The positive influences were 
adequate knowledge of SCD home care, access to health services, and adequate financial and material resources. Negative influences were limited knowledge of the pathology of SCD, concern about adverse community beliefs and negative attitudes, disease symptomatology and body image disturbances, difficulty in accessing SCD focused services, limited financial resources (often because of unemployment), the high care related costs and limited family support especially parental involvement in care. These findings are in accord with individual, society and programmatic vulnerability factors found among adult SCD patients in Brazil [174]. They also corroborate with contextual and vulnerability factors proposed by the theory of self-care management for vulnerable population [175]. In contrast to international findings, a key adverse contextual factor within the Ghanaian context was the ascription of metaphysical causes such as witchcraft and evil spirits to SCD and food related misconceptions especially in relation to consumption of fats and oils.

The statistical computations show that income did not pose a serious barrier to selfmanagement as there was no statistically significant correlation with income and the number of self-management actions an individual performs. Patients' and parents' other characteristics such as gender, rural versus urban residence, access to clinic, number of crises and education did not influence self-management practices. The results from the statistical analysis of income and access to SCD clinics contrasts with the qualitative findings as many participants described financial challenges and access to clinic as a major challenge. One possible reason is the variables used to calculate the total self-management actions for each participant was based solely on researcher selected self-management actions which did not include all responses provided by each participant. Secondly, many participants discussed financial and clinical access in relation to clinical care rather than self-management. Despite statistical evidence, the concerns expressed by patients in the qualitative reports are important to note for further research. The next sections discuss the safe and unsafe practices that were identified in the patients and parents reports.

\subsection{Safe and Effective Practises}

The findings indicate that most self-management practises were considered useful by patients and can be assessed as clinically safe. It is evident that the majority of practises were informed by health professionals, either at SCD clinics or through the mass media. The most clinically relevant findings relate to self-treatment and preventive health actions which are 
understandable since these are the major areas patients actively reported. This section highlights the commonest actions patients and parents indicated to be effective. I used my clinical experience and literature to determine the safety of the practises.

\subsubsection{Self-treatment}

Respondents generally found their self-treatment helpful (Table 18). Both quantitative and qualitative findings identified respondents used a combination of pharmacological and non-pharmacological strategies to tackle problems. Moreover, more participants (13/19 interviewees) considered the conventionally oriented self-management interventions usually informed by health professionals more effective than their traditional practices. These findings corroborate Ampomah Gyamfuah, Kumi-Kyereme, Darteh, and Addo's (2015) study that reported a changing preference among Ghanaians for orthodox health services due to availability of health professionals and equipment that makes the care more effective than traditional medicines. The most useful reported self-treatments for pain involved taking analgesics such as paracetamol and ibuprofen, applying heat, increasing hydration, massage and application of harsh topical creams to painful body parts. These results are consistent with research that identified pain treatment practices among SCD patients in the USA [116, 125]. These studies, reported that the majority of SCD patient combined analgesics with nonpharmacological strategies (CAM) to manage SCD pains. Both studies reported patients considered their interventions useful. This current study's findings are consistent with the Dampier et al. [116] findings. These authors recommended there was a need to strengthen patient and parent capacity to master effective application of pharmacological and nonpharmacological strategies in SCD pain management.

The respondents also reported successfully managing anaemia, fever, headache and stomach troubles. These results have not been described in previous research as almost all studies on self-treatment concern pain management. The most useful anaemia management strategies described were eating a nutritious diet or special diet, taking folic acid daily, and taking home prepared tonics. The most notable finding here was the combination of common vegetables, mostly involving Kwawunsusua (Solanum torvum). The frequent use of Kwawunsusua is not surprising as a widely held belief exists in Ghana regarding its potency for promoting haematopoiesis thereby, preventing or restoring anaemia. In Thailand, the West Indies and other African countries, this vegetable is used to manage a wide range of 
conditions including anaemia [240]. Although, no empirical evidence was located in current medical literature to the claimed efficacy in relation to anaemia, it can be assumed to be safe given it is a green vegetable which is likely to provide essential nutrients.

Respondents reported the use of antipyretic medication, tepid sponging, and antimalarial medication as helpful in fever management. These findings are consistent with studies that have explored fever management in the Ghanaian population [241]. These practices are clinically safe and follow recommendations made by the Ministry of Health. Malaria constitutes the major cause of febrile illness in Ghana and the MOH recommends self-treatment with anti-malarial medication and related therapies [242, 243]. It is possible that these results are influenced by the recommendations of the MOH. In the USA, SCD patients experiencing fever are recommended to report urgently to hospital without any form of home interventions for fear of severe infections [70]. The interviews with health professionals in this study revealed two different opinions regarding fever care at home. One opinion advised patients to use self-treatment such as tepid sponging, antipyretic and antimalarial medications. This opinion assumes fever is most likely due to malaria, rather than bacterial infection. The other opinion advised total avoidance of self-treatment and seeking a diagnosis for the cause of fever. These differing opinions indicate the need for the $\mathrm{MOH}$ to establish clear guidelines for managing SCD fever at home.

The study revealed simple measures can relieve patient's signs and symptoms. For example, applying fluids to the penis and exercises relieve priapism, and rest and sleep relieve painful or itchy eyes, bodily weakness, severe headache, and other painful events. A number of these findings are similar to studies that investigated CAM strategies in SCD management. In Dampier et al.'s [116] study, patients found sleep and relaxation effective in relieving pains including headache. The researchers thought that sleep and relaxation are comfort measures that help patients to cope with pain. Similarly, application of heat and massage were reported to relieve pain due to vasodilation [116]. Although these low cost interventions are clinically safe and their use can be encouraged among SCD patients, it is important to advice patients to seek professional's assistance if their symptoms recur to rule out serious underlying pathologies.

Other cost effective self-treatments and interventions reported to be useful by some respondents were the use of traditional medicines, including herbal medicines, prayers, and 
spirituality. The named herbs were ferma, tuatene, aguro, nkranpan, and nintini. These medicines were either mixed with food or boiled and administered as concoctions. Parents found these strategies useful for the general health of their children and in reducing the frequency of painful crisis and other illness. However, these parents also reported their inability to afford treatments recommended by the orthodox medical system. Therefore, it is unclear whether these parents use the traditional medicines based on their efficacy or affordability. Most respondents and participants reported topical traditional medicines effective for pain treatment. These were Maame Dagomba, Nompe-Nompe, Lord Balm, Apomden, and Shear butter (these medicines contain locally processed herbs). The safety of these medicines is currently unknown, despite their usefulness to patients as no empirical evidence exists to support their safety. The use of traditional medicines is common in Ghana, especially for chronic conditions that are inadequately managed or cured by orthodox healthcare. Therefore the findings of the current study among SCD patients is not unusual. A population survey in the study region to investigate prevalence of traditional medicine use among the people found that $86.1 \%$ of 324 respondents had used some form of traditional medicine [244].

The WHO (2008) estimates that in Africa about $80 \%$ of people use traditional medicines for primary health care. Traditional medicines are the most accessible health service options for many Ghanaians, especially those limited by geographical and financial access to orthodox health services. The medicines are usually in the form of herbs, plants or derivatives. Recent innovations have seen many of these medicines transformed into tablets, pills, tinctures and creams that are sold through commercial outlets in Ghana [245].

Another traditional strategy reported by respondents was the use of prayer and spirituality in coping with SCD related problems (Table 14). Four interview participants were also hopeful God will heal, cure their disease, or help them to cope with public ridicule due to SCD and $71 \%$ reported having resorted to prayer during illness. As many Ghanaians are religious [233], it is common for Ghanaians to explain difficult situations metaphysically or seek spiritual assistance. For example, Gyasi et al.’s [244] survey of traditional medicine practices in one district in the study region (Ashanti) showed that prayer accounted for $58 \%$ of commonly used therapies. The use of prayer and spirituality in SCD care is not peculiar to the Ghanaian context as similar findings were observed in studies that explored self-management practices in USA and Brazil [116, 125, 174]. Prayer and spirituality have also been 
demonstrated to be useful for SCD care [121, 246] For example in theUSA, Cotton et al. [246] found that adolescents with SCD, reported prayer enable them prevent illness, recover early from illness and promoted emotional comfort.

Some explanations have been offered for the mechanism by which prayer impacts on health. Prayer is believed to be a form of meditation that leads to positive changes in stress and improvement in health outcomes [247, 248]. The finding of the current study is consistent with recommendations for including prayer and spirituality in SCD related interventions [123].

\subsubsection{Self-monitoring and Diagnosis}

The findings show that simple physiological observation of physical signs and internal cues enable patients to recognise impending or actual problems for which they can take corrective action or seek medical attention. Some of these signs and cues were unique to individual patients and were not identified by the health professionals. This finding highlights the important role patients' experiences can contribute in the early detection and prevention of complications. The findings corroborate other studies in the USA where monitoring enables early diagnosis and management of problems among SCD patients [131, 132]. However, the self-monitoring and diagnosis in the USA studies were based on researchers' prescriptions whilst the findings from the Ghana study are from patients perspectives.

\subsubsection{Preventive and Maintenance Actions}

A range of preventive health and maintenance actions were safe and effective for the patients. The most beneficial actions from patient's perspectives were frequent oral hydration, avoidance of cold, moderation in activities and exercises, and malaria prevention using barrier methods. Specific examples included eating balance diet, preparing fluids with local spices and vegetables, regular intake of folic acid, keeping warm, moderate keep-fit exercises. Patients indicated these actions reduce the frequency of illness. Although these actions appear clinically safe with physiological plausibility, it is worth noting that, to date, most of these are based on theoretical and intuitive reasoning with limited empirical evidence among people with SCD. For example the scientific merit of the efficacy for routine folic acid supplementation for manage anaemia in SCD is a subject of debate [249, 250]. Secondly, systematic reviews have shown no direct evidence for the efficacy of hydration, avoidance of 
over activity and extremes of temperature in preventive SCD crisis and other complications [251].

Despite the lack of strong empirical evidence, there are theoretical reasons to warrant the promotion of these measures among SCD patients. For example, SCD is associated with increased risk for dehydration, increased blood viscosity and acidosis as a result of increased diuresis secondary to hyposthenuria. These abnormalities can result in increased sickling, vaso-occlusive crisis and related problems. Consequently, increasing fluid intake is promoted to reduce the risk of dehydration [252]. These authors further assert that strenuous exercises can result in tissue anoxia, dehydration and stress which can precipitate painful crisis, hence moderation in exercise is recommended for SCD patient. Regarding cold temperatures, researchers have found that SCD patients have higher propensity for vasoconstriction induced by skin cooling, The frequency of painful crisis was more common among people prone to cooling induced-vasoconstriction than those not prone $[253,254]$. However, a recent systematic review did not find direct correlation between cold temperatures and SCD crisis [252]. Despite the lack of direct evidence linking prevention of SCD and hydration, cold temperatures and strenuous activities, the researchers did not identify harm associated with their use. Consequently, given that patients reported these measures useful, it is will be important for SCD self-management education to provide advice on the use of these measures.

\subsubsection{Health Professionals' Advice}

One of the significant observations in this study was the reporting by patients that consultation with health professionals and receiving advice improved their conditions, making living with or caring for SCD easier. Respondents made these observations when they compared their current situation to the previous situations when they used traditional practices. The reported benefits include reduction in the psychological burden of the disease, a sense of hope to patients and parents, reduction in frequency of crises, and ability to manage some minor conditions at home. Similarly, Jenerette et al. [17] found participants described the importance of having knowledgeable health professionals and following their advice. However, unlike the current study, the participants in the previous study did not describe the benefits they derived from following the doctors' advice. The positive impact of the interactions between patients and health professionals is well documented in LTC self- 
management literature $[10,13]$. These interactions result an increase in health literacy on the part of the patient and enable patients with LTCs to solve current and future problems [255]. Hernandez [254] further asserts that health literacy impacts the ability to access and use healthcare, to interact with providers, and manage self-care. The findings indicate that a patient health literacy programme designed around self-management education can enhance positive patient outcomes.

\subsection{Unsafe Practises}

There were potentially unsafe practises involving misuse of medicines, non-medicinal therapies, unsafe treatment for specific complications, and omission of essential activities. Although few instances of these practises were cited, it is important to identify those that can have significant implications for patient health.

\subsubsection{Misuse of Prescribed and Over-the-Counter Medicines}

The patients reported various forms of medicines misuse, and the use of recreational drugs. Medicines commonly misused were folic acid, antibiotics, analgesics, haematinics and traditional medicines. Specifically, the misuse included using the medicines for the wrong indication, under-dose or over-dose, missing dose, inappropriate combinations of medicines and use of strong narcotics (e.g., pethidine). The recreational drugs concerned use of alcohol and cannabis.

The findings identified that, rather than taking folic acid routinely as a supportive medication, some respondents used it as an analgesic, when they experienced crisis. Others reported taking higher than the prescribed doses of various medications. The misuse of folic acid could be unsafe given that decreased intake of folic acid in SCD may lead to depletion of folate stores and intensify the symptoms of anaemia, whereas increased intake may lead to completions such as increased episodes of priapism, increased risk for malaria and infections and hyperhomocystenemia which can lead to thrombolytic events and cause crisis [250].

The findings also report antibiotic misuse, commonly involving tetracycline, Amoxyclav and amoxicillin, for managing conditions such as painful episodes, leg ulcers, fever, and abdominal swelling. These findings are not surprising as inappropriate use of antibiotics is common in Ghana, and is thought to be linked to economic, health related factors, and availability. For example, a study found that $70 \%$ of tertiary students had self- 
medicated with antibiotics. The main reasons for the abuse were the lower cost compared to the hospital cost and long waiting hours in hospitals [256]. Misuse of antibiotics by SCD patients is a concern as patients can receive multiple antibiotic therapies during their life due to poor immunity [79, 257] leading to antibiotic resistance and hypersensitivity [258].

Another important finding for clinical consideration is the use of haematinics from both conventional and traditional sources reported in the interviews and survey. This finding reflects common practices with use of haematinics for the management of anaemia and loss appetite in the Ghanaian population [259]. Conventional haematinics are mostly iron rich and among SCD patients, self-medication may lead to iron overload and related problems unless the patient is experiencing iron deficiency anaemia [79]. Traditional sources of haematinics are herbal sources sold through retail shops in Ghana. These medicines may be unsafe for SCD patients as many contain less than the recommended dose of trace elements such as iron, magnesium, copper and zinc and some contain toxic metals such as cadmium and lead [259]. This findings indicates a lack of knowledge about the pathophysiological mechanisms of anaemia in SCD and effective methods addressing this knowledge gap are required.

There were also references to unsafe practice related to the use of medicines for the wrong indications. These include using antimalarial medications to treat jaundice and fever, haematinics to treat jaundice, eye-drops to treat headache, frusemide for priapism, and daily use of paracetamol as a preventative medication for pain. These medicines were often obtained from previous hospital appointments or obtained from other patients or relatives.

The findings also identify the use of non-prescribed medicines including food and vitamin supplements, immune boosters, and traditional and alternative medicines for common conditions such as fever, pain and anaemia. The use of similar bio-chemical products have been reported among SCD patients and similar long term conditions in studies that investigated CAM. In Nigeria, one study reported $58 \%$ of parents had used similar biochemical CAMs to manage asthma, epilepsy and SCD. The reasons for use were similar to those reported in the current study including: to treat or cure the disease, improve physical condition or relieve symptoms. Among SCD patients the major reason cited were to improve body immunity. In the USA, studies reporting CAM use among SCD patients also found use of herbal and alternative medicines [121, 125]. 
Although food and vitamin supplements and other bio-chemical products may have a role for health promotion, the safety of many of these have not been identified among SCD patients. Therefore, non-prescribed use of such medicines among SCD patients is a concern. As SCD patients are likely to be taking prescribed medications, non-prescribed food supplements and other bio-chemical products can result in adverse drug-drug or drug-food supplements interactions [260]. For example one interviewee reported experiencing serious complications after consuming a food supplement donated by a friend to boost immunity.

Other potential unsafe practices related to the use of traditional medicines to treat pain and other conditions. Although the majority of patients reported discontinuing traditional medicines, this is counter to health professionals' experience of patients' frequent use of herbal medicines. Of major concern is that most traditional medicines used by patients were raw herbs usually mixed with other substance that were applied topically, orally or rectally. Such routes of administration of herbs may present significant risk, as they can involve incisions on the abdomen and inserting medicines to treat splenic enlargement, or administering an enema of raw herbs mixed with spices. These practices can result in adverse drug reactions, enema retention and gastrointestinal irritation or infections such as tetanus from unsterile techniques. The reported use of traditional medicines is a concern as interview participants reported serious adverse reactions and although patients claimed to have stopped taking traditional medicines, health professionals were aware they continued to take processed and packaged herbs for topical application. One third of respondents reported having taken unknown processed and packaged herbal medicines to treat painful episodes. Finally, it was observed that the rationale for administering some traditional medicines did not have physiological plausibility such as administering of an enema to treat abdominal distention, caused by organomegally.

The results also identify differences between health professionals' observations of traditional medicines use and patients' reported use. Whilst health professionals' observations indicated frequent use, patients reported limited use. One explanation for this difference is that health professionals made these observations during their encounter with patients at SCD clinics, where patients may have discontinued using herbs based on advice received at the clinic. Alternatively, patients realised that the orthodox medicine was more effective than traditional medicines. Apart from traditional medicines, few alternative medicines were 
mentioned, including Chinese medicines for which patients had no knowledge about their kind.

The use of traditional medicines for self-treatment in SCD appears to be a common practice which is not limited to Ghana. The use of several herbal products have reported for the treatment of sickle cell disease in Africa [261]. In the USA, two studies that investigated CAM use among pediatric population found that herbal medicines were used by $6.9 \%$ [125] and $12 \%$ [121] of respondents.

The major challenge with use of traditional medicines in Ghana and many nations relates to lack of evidence of safety [259]. Internationally, there is ongoing research to identify efficacious and safety of traditional medicines for SCD [262-264].

To date, only two phytomedicines (Ciklavit, Niprisan) have been scientifically shown to reduce severe painful crises [262, 265, 266]. However, these two medicines have limited impact in preventing complications and the safety of Ciklavit is unclear; researchers recommend use of this medicine with caution [264]. Ghana has an opportunity to scientifically explore the role of traditional medicine in SCD care due to the efforts by the $\mathrm{MOH}$ to formalise traditional medicine in the health system and the existence of the Centre for Scientific Research into Plant Medicine [47].

Another potentially unsafe practice is the use of recreational drugs such as alcohol, cannabis and prescribed opioids. For example, one interviewee reported combining a strong local gin (Akpeteshie) with paracetamol to combat pain and promote sleep. In the survey, four (1.6\%) respondents reported using alcohol or cannabis to manage SCD pain (Table 14). International studies that have reported alcohol [119] and cannabis [117] use among SCD patients attending clinics in the USA and UK found higher proportions of recreational drug use than the current study. Levenson et al. found $31 \%$ of SCD patients had used alcohol and Howard et al. found $36 \%$ had used cannabis. The very low number of reports among respondents in the current study could reflect under-reporting since cannabis use is illegal, and consumption of alcohol is associated with antisocial behaviour in Ghana, and my experience is that SCD patients are advised to avoid alcohol use.

Two interview participants reported self-treatment with strong opioids (pethidine) for their leg ulcers. Misuse of opioids appear to be a common feature among patients with 
chronic pain [267]. However, limited studies have been reported opioid use among SCD patients. A study in Nigeria reported opioids addiction (pethidine, pentazocine) among a cohort of 11 SCD patients (13-53 years) attending hospital [268]. Kotila et al.'s findings have some similarities with the current study in that they found opioid use among patients with chronic pain. However, the current study found pethidine use among people with leg ulcers, while Kotila et al. reported the use among patients with avascular necrosis of head of femur, cholelithiasis and stuttering priapism. Similar to the current study, Kotila et al. reported that patients use tricks such as forging prescriptions. As strong opioid use is associated with chronic pain, research is required to further investigate this practices among SCD patients.

\subsubsection{Non-medicinal therapies}

An important finding related to non-medicinal practices that could pose a risk to safety is the application of a cold compress, or the use of tepid sponging or cold baths to treat a painful body part. In SCD, the application of cold can result in local vasoconstriction and exacerbate painful episodes [252]. It is my experience that patients should be advised to use lukewarm water to reduce the risk of inducing crisis.

Another aspect of unsafe practices relates to the use of mechanical measures including binding, applying weight, and scarification on affected part. Similar practices have been reported by a Ghanaian newspaper that investigated traditional for SCD care in rural communities [269]. The practices can be unsafe given that binding or applying weight to a painful part can occlude blood vessels and interfere with circulation to the affected parts resulting in ischaemia, pain and tissue damage. Although few patients and parents reported using these measures, it is necessary to draw attention of the existence of such harmful practices in SCD management especially since the Ghanaian health professionals had concerns with these practices.

\subsubsection{Unsafe Treatment for Specific Complications}

Unsafe practises regarding the self-management of abdominal distention, hip problems and leg ulcer deserve attention. The use of medicines (e.g., antibiotics, antihelminthics, metronidazole, anti-acids, multivitamins, topical creams, enemas) and non-medicinal strategies (e.g., warm compresses, scarification) to treat abdominal distention is problematic and is likely to be influenced by the lay perceptions of the symptoms. In Ghana, my 
observation is that abdominal distention is usually attributed to worm infestations, accumulation of phlegm's or supernatural machination. Therefore, the practises observed in this study are likely to be directed at solving these problems and while these interventions are not likely to result in benefits to the patient, they can cause problems. As an example scarification with unsterile equipment can cause bleeding and infection.

The use of vigorous exercises such as jumping to manage hip problems can potentially complicate or induce hip problems [79]. The use of domestic equipment such as buckets, hot water, bath towels, and the application of topical antibiotics, metronidazole powder or grounded herbs without regard to asepsis can result in infection, hypersensitivity and antibiotic resistance. These findings have not been researched, and raise implications for future research and health education.

\subsubsection{Omission of Essential Practices}

Two areas of omissions were identified in the patients reported practices. A few respondents indicated non-engagement with self-treatment as they attend clinics for all problems. Another group were unwilling to attend clinics preferring to engage self-treatment. For example, two participants preferred to provide traditional medicines to their children, and two adults prefer to manage their leg ulcers and priapism at home. These two variances in engagement with self-treatment constitute omissions that are unsafe because SCD management, like all chronic conditions, requires a balance between self-management and interactions with health professionals.

The avoidance of staple foods such as $f u f u$ (pounded plantain and cassava eaten with soup), rice, beans and peanuts was due to lay beliefs. In contrast, findings in the USA did not identify evidence of food restrictions for SCD self-management [17, 123, 176]. Rather, there was endorsement of maintaining a healthy diet by SCD patients. Unwarranted food restrictions can be unsafe for SCD patients, especially children who require extra calories to compensate for the constant energy lost due to rapid haemolysis resulting in malnutrition, growth impairment or anaemia [79]. Nutritional education which emphasises the role of nutrients in the body is required to address this food related misconceptions in Ghana.

Other omitted actions relate to self-monitoring and diagnosis among patients. Contrary to expectation, approximately a quarter of respondents and two parent interviewees made 
reference to monitoring temperature and only $6.9 \%$ of respondents had thermometers. The studies from the USA which investigated self-management practices in SCD patients [17, 123] did not report temperature, possibly because the participants were adults and in whom fever is not a concern. This result is of major concern given the importance of fever in children. In Ghana, infections in SCD patients related to malaria, are major causes of mortality and morbidity $[4,59]$. A possible explanation for the limited use of thermometers and temperature checking is that lay people consider these actions diagnostic which they should use only when they or their child is sick.

\subsection{Self-management of SCD and Self-management Theory}

The study conceptualised self-management as actions including preventive health, self-monitoring, self-diagnosis, self-treatment and self-evaluation; factors influencing selfmanagement as contextual, vulnerability and self-management resources; and outcomes of self-management as health status and QOL. The framework (Figure 2) successfully guided the data collection, analysis and interpretation of the research. In this section, I evaluate the framework relative to diabetes and hypertension and its application to SCD.

Preventive health, as observed in this study, is consistent with reviewed frameworks and literature on LTCs such as diabetes and hypertension [23, 26], although the specific actions differ. It mainly concerns health promotion and prevention of disease exacerbation, and maintaining interactions between patients and health professionals.

However, the focus of self-monitoring between SCD, diabetes and hypertension differs. In diabetes and hypertension, self-monitoring mainly involves self-measurement of physiological variables (blood glucose, blood pressure) using physical equipment [180]. In contrast, as demonstrated the findings of this study and other reviewed studies, selfmonitoring in SCD emphasises physical sensations, observations of signs, and tracking triggers of disease exacerbation. There is limited focus placed on measurements of physiological variables and use of physical equipment.

Limited literature exists to enable comparison of self-diagnosis as the term has limited application in self-management research. However, if the operational definition of this study is used as the criteria (recognition of problematic signs and symptoms or deviations) it can be argued that self-diagnosis in diabetes and hypertension focus on self-interpretation of 
objective physiological data [180]. This will involve self-recognition of abnormal values for blood glucose and blood pressure. In contrast, SCD self-diagnosis is based upon patients' subjective interpretation of physical sensations or signs.

Self-treatment pertains to phenomena common to SCD, diabetes and hypertension involving administration of pharmacological and non-pharmacological therapies. However diabetes and hypertension, self-treatment focus on self-adjustment or self-titration of medications and diet, administered on a daily-basis, and requires strict adherence to specific medication and dietary schedules [180, 181, 183]. Self-management in SCD less prescriptive, may not occur on a daily basis. There are little emphasis on use of non-pharmacological strategies for therapeutic reasons. In contrast, self-treatment in SCD largely involves administration of pharmacological and non-pharmacological measures to combat a recognised problem.

Factors influencing self-management are largely consistent with other conditions involving individual and environmental (contextual) factors as postulated by self-management theories $[19,32]$. In the current study, a key contextual factor is lay perspectives that form stigmatisation and labelling of patient and their families affected by SCD. Conditions such as diabetes and hypertension are not generally recognised as being associated with such stigma.

\subsubsection{Reflection on the conceptual framework}

The framework adequately covered the data with minimal deviations. It was useful for data collection analysis and interpretation. However, the framework has been revised in light of this study's findings for future researchers.

Reflecting on the conceptual framework I appreciated that the initial framework was illness or disease focused. It assumed that patients perform only disease related actions (preventive health, self-monitoring, self-diagnosis, self-treatment, self-evaluation). However, the findings of the study show that in addition, patients manage their SCD by performing other actions that are related to living with SCD (many of these were psychosocial in nature). Other actions include managing emotions (prayer, religiosity, positive self-concepts, role models, comparison), managing biography (work, love, roles, education), seeking collaboration, seeking information from professional and lay sources, seeking support from family and sickle cell associations and consultation with health professionals. These actions 
may directly or indirectly influence disease management and outcomes. These actions have been incorporated into self-management resources in a revised framework (Figure 4) as they concern the everyday resources that people may have in their lives. The findings also show that the factors that influence self-management are mainly the contextual and vulnerability factors.

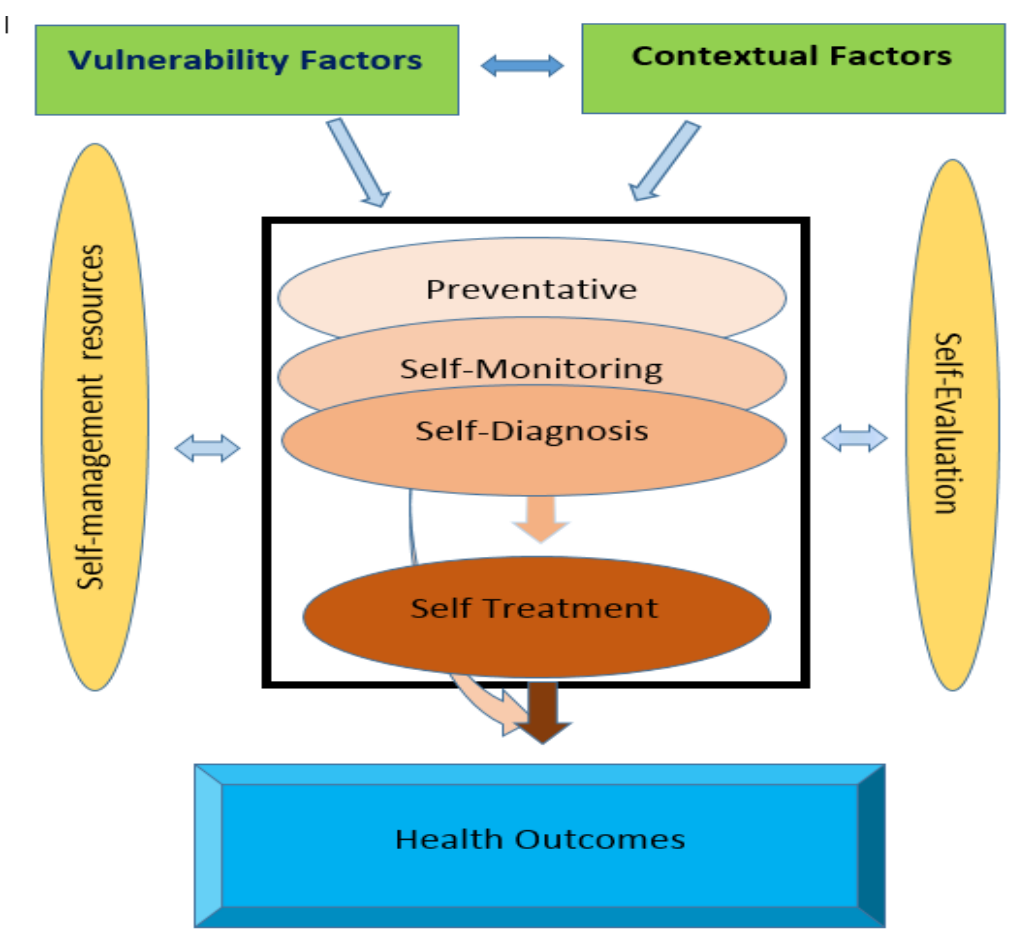

\section{FIGURE 4. REVISED CONCEPTUAL FRAMEWORK FOR SCD}

Three other changes were made to the initial framework. These concern the position of where self-management resources sit; the interrelationship of prevention, monitoring and diagnosis, and the position of self-evaluation. Positioning self-management resources horizontally on the left of the closed box of actions signals that resources are an essential part of taking actions. Framing these resources as part of self-management actions, as opposed to factors also reflects that these are actions that concern process, as opposed to background factors in the earlier framework. The acquisition and utilisation of self-management resources such as knowledge of SCD and seeking social support were seen as part of self-management actions.

The revised framework also demonstrates a linkage between preventive health, selfmonitoring and self-diagnosis. The linkage between preventive health and self-monitoring was demonstrated by statements such as "I watch the food I eat"; "I ensure that I don't eat rice 
cooked with oil in the night". The word "watch" refers to self-monitoring. It implies patients keep track of food items with the intention of identifying those that can trigger crisis. This statement can also imply preventive health. The "watch" could mean being careful or selective of choice of food which indicates preventive health. The linkage between selfmonitoring and self-diagnosis was demonstrated in the statement "I normally get the indication that I am about to get sick when I am tired easily climbing hills". This statement indicates patients can be in tune with listening to bodily cues (self-monitoring). It also signifies the patient's ability to recognise a deviation from normal (self-diagnosis). Such situations presented challenges with coding and categorisation. Future researchers may need to clarify what is distinct and what overlaps between preventive health and self-monitoring as well as between self-monitoring and self-diagnosis.

It was observed from the data that self-evaluation also occurs as part of prevention, monitoring and diagnosis, contrary to the proposition of the initial framework, which situated evaluation after self-treatment. In prevention, patients tended to continue with helpful actions and discontinue unhelpful ones, and in how they monitored and recognised an accurate diagnosis. Given this finding, self-evaluation has been moved to a horizontal position and located on the right hand side of the boxed actions.

Regarding the self-management outcomes, it was a challenge distinguishing between self-rated health and QOL using the Ghanaian local language, which made it difficult for some people to distinguish between these in the quantitative survey. In the future, a multiquestion approach will be more useful in eliciting these concepts than single scales in settings similar to the Ghanaian context.

\subsection{Limitations of the Study}

The limitations of the qualitative phase mainly involves data collection. It is possible that some relevant websites might have been excluded from the sample for the website review as the study purposively selected from approximately the first 100 outputs and was limited to only English language sites. Secondly, some relevant texts might have been omitted or overcounted as data was manually collected and counted. The limitations regarding the interviews with health professionals, patients and parents mainly concern challenges with phone interviews. Due to limited telephone coverage in Ghana, some potential candidates could not be reached especially those in the rural areas, therefore the perspective obtained is from those 
mainly living in urban areas. It is also possible that some patients and health professionals may have exaggerated what they did. Patients in particular may have not been truthful information on sensitive topics such as use of use of recreational drugs and traditional medicines. These factors might have impact on the diversity of information obtained.

The quantitative phase has three main limitations - selection of the samples, statistical analyses and use of self-reported data. The use of a non-probability (consecutive) sampling to select the survey participants limits the generalisability of the findings. However, the attempts to recruit all eligible patients and parents within the study region, during a limited period of time did result in a sample size of 313 patients and parents. This represents $74 \%$ of the calculated sample size of 422 . However, the sample obtained is sufficient to reflect the perspectives of the targeted population. Therefore, the results have wide applicability and will benefit SCD patients, caregivers and health professionals. Moreover, the sample did not include patients without access to SCD clinics and the SCD Association. Given the significance placed on health professionals' advice, patients and parents not able to access services may practice different self-management. However, the inclusion of questions to elicit lifetime SCD self-management experiences indicated practices prior to accessing SCD clinics.

The second limitation related to statistical analysis of the survey data. The computed figures for each respondents' total self-management actions could be underestimated as the computation included only the researcher selected items (pre-determined items). The computation did not include individual responses from the open ended questions as some questions were not applicable to all respondents. This risk of underestimation was reduced as in the analysis the researcher selected the most frequent variables from the participants' narratives to develop scales that were applicable to all participants. Individual responses from these scales were then used to calculate the total self-management actions for each respondent. A related limitation was that statistical testing was not undertaken on all sociodemographic variables to establish any relationships between self-management and sociodemographic characteristics.

The third limitation relates to potential for respondent bias inherent in self-reported data, such as recall bias. The risk of respondent bias was reduced through the use of structured questions in the survey, limiting the recall period to the past 12 months, presenting the questions in the local language, and allowing sufficient time for participants and respondents 
to adequately capture their memories. In general, the use of different data collection methods to investigate individual's self-management mitigated the risk of respondent bias. For example, the interviews not only informed the surveys but were also discussed alongside those of the survey to check the congruence of the reports.

\subsection{Implications and Recommendations}

The literature review and study findings have shown that self-management practices are helpful for improving the health of SCD patients and can reduce the burden on families and pressure on health resources. However, self- management must be utilised in appropriate ways to generate favourable responses and decrease unfavourable outcomes. The following implications draw attention to what the findings mean and the recommendations point to what needs to happen to improve the self-management of SCD, and other long term genetic conditions, in Ghana as well as for patients in other countries.

\subsubsection{Implications for International and Local Policy}

The current study identified the dimensions and benefits of SCD self-management in Ghana. However, for self-management to become usual practice in countries such as Ghana, it must be promoted by national and international organisations through advocacy and establishment of deliberate policies. This will require the WHO to establish best practice for self-management of SCD to serve as a guide for country specific programmes. It will also require the WHO and related international bodies to include SCD self-management support in their policy recommendations for SCD management in countries with high prevalence.

Given the finding of a high utility of self-management education for the well-being of SCD patients, there is a need to strengthen Ghanaian health professionals' capacity to offer support to patients to promote effective self-management practices. The Ministry of Health should consider including self-management support in SCD care. Delivering this support will require consensus of topics and skills required by health professionals. Key priorities should aim to reduce unsafe practises and enhance safe and effective practises, provide technical information to supplement patients' experiential expertise, and teach new knowledge and skills. The MOH should provide clear guidelines for home management of fever for SCD management and include in the practitioners Standard Treatment Guidelines [114]. Air travel precautions and avoiding low oxygen environments were common recommendations 
identified in websites but not described by the health professionals or patients. In view of the proliferation of domestic airlines in Ghana, it is important for the $\mathrm{MOH}$ and the Ghana Civil Aviation Authority to consider policies on precautionary measures for SCD patients travelling by air.

The Ministry of Health should develop and implement culturally appropriate SCD self-management programmes and interventions within the public health systems. Such programmes will be useful in settings with limited SCD focused services. The selfmanagement variables identified in this study could serve as an entry point to the SCD selfmanagement programme. The MOH could collaborate with the Sickle Cell Association of Ghana (SCAG) and the Sickle Cell Foundation to establish the programme. The SCD selfmanagement programme could be led by professionals providing technical support or peer led in some settings and either located within ambulatory care where SCD clinics exist or run by voluntary organisations such as the SCAG.

\subsubsection{Recommendations for SCD Association}

The study has identified that websites can provide information to patients and their families and health professionals to acquire useful knowledge for self-management of SCD. It is recommended that the SCAG consider the development of a website to promote selfmanagement. This site can take advantage of technological advancement to include text, videos and other illustrations for patients and parents who cannot read and write. It must include interactive options to create a platform to address concerns. A site focusing on patients' education should provide information on self-management with tailored information specific to audiences such as children, adolescence and adults. The focus of the information should centre on promoting safe and effective practices and reduction of unsafe practices. Sites can use the information generated from the current study to update their patient focused information and should ensure that recommendations are devoid of technical language.

\subsubsection{Recommendations for Nurses and Other Clinicians}

The findings have recommendations for nursing practice, education, research and theory. Self-management for LTC in the international literature is a nursing dominated area. Nurses in Ghana and similar countries can equally take leadership roles for SCD and other LTC self-management. 


\subsubsection{Recommendations for nursing practice}

Nurses' roles at the clinical and community level provides them with better opportunity to lead self-management interventions than the other team members. At the practice level, nurses can utilise the findings to improve their advice to patients, offer support to carers, and promote positive public perceptions of the disease. The Nursing and Midwifery Council should collaborate with the Ministry of Health to include self-management support services for SCD care. It is recommended that nurses and other clinicians teach patients to acquire preventive health and maintenance strategies that include: hydration, nutrition, folic acid supplementation, safe activities, avoidance of extreme temperatures, vaccination nutrition, air travel precaution, personal hygiene, avoiding low oxygen environment, malaria and infection prevention, medication, triggers, and coping strategies. Achieving this recommendation will require recognition of the value of self-management as well as extra time. The long term benefit however should be a reduction in the number of crises and patients with SCD being able to live fuller lives.

Patients' self-monitoring, which has been identified in literature as the most important self-management strategy, was used ineffectively by most respondents. Therefore, nurses should teach patients proactive self-monitoring and essential physical assessment skills. Key topics should include fever measurement, examination for jaundice and pallor, palpation for splenic enlargement and other abdominal viscera for organomegally, pain assessment, triggers identification, growth monitoring, monitoring internal cues, observation of behaviours, explanation of laboratory results and other medical examinations, and evaluation of new treatments. Nurses should provide and teach patients and caregivers the use of self-monitoring tools and devices such as thermometers, pain scales, weight and height measurements, and explore the possibility of use of computers and ICT such as videos and text messages to support patients. Emphasis could be put on value of journal and diary use.

The findings demonstrate a discrepancy between patients' and health professionals' interpretations of emergency signs or symptoms and therefore teaching effective selfdiagnosis is a priority. Nurses can use the variables identified in this study to develop checklists self-monitoring and diagnosis. It is recommended that nurses teach patients and caregivers how to treat minor ailments including first aid management of common SCD related problems. This requires an agreed protocol to address medicines safety, effective 
methods of utilising non-medical interventions, as well as equipment and resources to facilitate self-management. Nurses need to integrate the CAM strategies identified as effective by patients and parents into the care of SCD patients. Due to limited access to SCD clinics in Ghana, nurses could develop distance support strategies such as telephone advice and online prescribing and dispensing. Nurses should ensure that the spiritual and other psychosocial needs are addressed when delivering self-management support. During history-taking clinicians should obtain information about patients' self-management practices and provide advice as necessary regarding unsafe practices.

The study has identified that professionals were the main source of self-management information and support. Therefore nurses and other professionals should to be conversant with other sources of information available to lay people such as the SCD focused websites and identify those that offer the appropriate recommendations for their patients. Nurses and other professionals might consider accrediting websites and other resources and encouraging their patients to use those sites.

Nurses practising in the community have an important role in correcting misconceptions related to SCD. It is pertinent for nurses in the community and other public health officials to educate the population to promote bio-medical understanding of SCD and the availability of effective self-treatment options. Nurses should reinforce teaching with aids such as audio-visual and leaflets based on the age and readability level of the targeted audience.

\subsubsection{Recommendations for nursing education}

The Ministry of Health should collaborate with Nursing Faculties to include selfmanagement for SCD and other LTC such as HIV conditions in the curriculum of undergraduate and postgraduate nursing programmes similar what is being pursued in other countries such as Australia [270]. The nursing directorate of $\mathrm{MOH}$ and GHS could include self-management training in structured in-service programme as part of nurses' professional development. Given the high prevalence of SCD in Ghana, the Ghana College of Nursing, should consider training certified SCD specialist nurses (like the US) as part of their haematological nursing programme [271]. These nurses can then hold nurse-lead SCD clinics and lead self-management interventions for SCD. 


\subsubsection{Implications for nursing theory and research}

Ghanaian nurses should implement and test self-management interventions for SCD. The Ministry of Health should include self-management research in its research agenda for SCD and communicate this to nursing and other health faculties. This could involve researchers to develop a theory to to serve as a foundation or model for SCD self-management for the Ghanaian context and similar systems. Such a theory will provide a direction for hypothesis, research questions and unify research studies addressing SM in Ghana. The concepts identified in the current study can service as a basis for developing and testing theories.

The conceptual framework indicates that self-management for SCD is complex and comprises many interrelated components. However, what ate the essential components is unknown. Nurses undertaking research and theory could break the various components of self-management down and research individual components to identify where the priority should be in the face of limited resources. For example, as reported in the literature review, researchers in SCD self-management studies in the USA have focused mostly on selfmonitoring. However, the findings among Ghanaian professionals and patients in the current study indicate that more emphasis is placed on preventive health. Nurse researchers should clarify if focus on self-management has contextual differences in order to direct appropriate interventions.

\subsubsection{Recommendations to Patients and Carers}

Patients and carers can do considerable work a lot on their own to control the impact of SCD on their or the SCD patients' lives. Patients should actively engage in learning and practicing of safe and effective self-management skills and avoid unsafe practices. Patients can also take advance of existing self-management programmes such as the Chronic Disease Self-Management Programmes and Expert Patients Programme being offered for lay people through distant learning to acquire self-management knowledge and skills.

Patients are encouraged to clarify their misconception with health professionals. They should collaborate with health professionals to identify additional sources of self-management information and support. Patients and carers should support and participant in selfmanagement programmes and research. Individual patients or patients groups such as the 
Sickle Cell Association of Ghana can advocate for the establishment of self-management policies and interventions. Patients should assist in forming and strengthening support networks such as the SCD Association and utilise this in sharing success stories about safe effective self-management

\subsubsection{Implication for Future Research}

Future research should build on the results of this study, address limitations of the study, establish relationships that were not tested, and build on the conceptual framework. Firstly, the study demonstrated a number of non-pharmacological strategies (e.g., hydration, nutrition) useful to patients that have not been researched. It is recommended that research involving more respondents, more rigorous methods, representative sampling be carried out to confirm efficacy and appropriateness of non-pharmacological strategies to improve patient outcomes. A similar approach should be used to clarify the efficacy, safety and affordability of traditional medicines and other CAM strategies claimed to be useful by patients.

Furthermore, previous researchers have demonstrated that the use of diaries and other ICT are helpful for patient self-monitoring. Given the current proliferation of mobile and internet technology in Ghana, researchers could explore such modern strategies to enhance SCD management.

Secondly, the study identified some harmful practises that can negatively affect patients' health. Further research is required to explore these practises with an aim to reducing the misuse. For example, clinical teams should investigate misuse of common medicines such as penicillin $\mathrm{V}$ and folic acid. Thirdly, research should address the sample limitations of the current study by capturing the practices from patients with limited access to SCD clinics. This is important given the finding of reported differences in patients' traditional and current practices after an encounter with SCD clinics. Patients in the study region (Ashanti) are likely to be exposed to SCD related interventions more than people in Ghana because of its history with Newborn Screening Programme. The region also has the most viable SCAG. Consequently, the people in the Ashanti region may have been exposed to more bio-medical teachings than those in other regions.

Fourthly, research should be conducted to explore differences in self-management strategies from the perspectives of children under 16 years and also of families of adult SCD patients. Finally researchers should establish the relationship between patients with paternal 
involvement and those without, and the relationship of religiosity and spirituality in selfmanagement. A more rigorous correlation study using more complex statistical may establish relationships and dominant factors that can predict self-management.

\subsection{Statement of Conclusion and Contribution}

Much has been learnt from websites, professionals, individuals who manage SCD in various settings. The findings indicate a consensus of the scope and dimensions of SCD selfmanagement with limited variations from the four data sources. The variations are largely influenced by the level of health service inputs for self-management support. Findings provide an understanding of the common self-management strategies utilised in Ghana; and those that are safe and effective, unsafe, or ineffective. We also understand which strategies are more common for adults and children, and the key factors that may influence people's selfmanagement practices. This study extends our knowledge as it included the broader perspectives from different players including adult patients, parents, professionals, websites, rural and urban respondents in a single study compared to previous SCD self-management studies which mostly focus on either adults or children. In addition, the study has contributed to knowledge regarding self-management practices for SCD related problems including fever, leg ulcer, anaemia, priapism, abdominal swelling, jaundice, hip problems, which have not been previously reported. The study provides insights into cost effective and simple selfmanagement strategies that can be promoted to improve the health of SCD patients. Furthermore, the findings provide considerable information that could help inform policy towards the development of a culturally appropriate, locally suitable, SCD self-management intervention programme in Ghana and other countries with similar health systems.

The findings add to the growing body of literature on SCD self-management. It especially contributes to filling knowledge gaps in the literature regarding self-management practices in low and middle income countries for genetic conditions in general and SCD in particular. The conceptual framework and the survey instrument produced from the study can serve as a base or contextualised for future studies on SCD self-management and similar conditions in Ghana and other countries. The study also has significance to health workers who work with people with chronic conditions, especially genetic conditions. As selfmanagement related concepts such as self-diagnoses, self-treatment has limited application in LTCs self-management literature, this study has contributed in operationalising these concepts 
in an empirical research. The study has contributed sequential mixed method design. As most sequential designs are two stage, this study has demonstrated how more than two stages sequential mixed method may be conducted. 


\section{Appendices}

\section{Appendix 1. Victoria University of Wellington Ethics Approval}

\begin{tabular}{|c|c|c|c|}
\hline \multicolumn{2}{|c|}{ MEMORANDUM } & $\begin{array}{l}\text { Phone } \\
\text { Fax } \\
\text { Email }\end{array}$ & $\begin{array}{l}0-4-4635676 \\
0-4-4635209 \\
\text { Allison.kirkman@vuw.ac.nz }\end{array}$ \\
\hline TO & \multicolumn{3}{|l|}{ Andrews Druye } \\
\hline COPY TO & \multicolumn{3}{|l|}{ Kathy Nelson } \\
\hline FROM & \multicolumn{3}{|c|}{ Dr Allison Kirkman, Convener, Human Ethics Committee } \\
\hline DATE & \multicolumn{3}{|l|}{2 May 2014} \\
\hline PAGES & \multicolumn{3}{|l|}{1} \\
\hline SUBJECT & \multicolumn{3}{|c|}{$\begin{array}{l}\text { Ethics Approval: } 20849 \\
\text { Self management strategies for sickle cell disease in Ghana }\end{array}$} \\
\hline
\end{tabular}

Thank you for your application for ethical approval, which has now been considered by the Standing Committee of the Human Ethics Committee.

Your application has been approved from the above date and this approval continues until 26 February 2016. If your data collection is not completed by this date you should apply to the Human Ethics Committee for an extension to this approval.

Best wishes with the research.

Allison Kirkman

Human Ethics Committee 


\section{Appendix 2. Ghana Health Service Ethics Review Committee Approval}

\section{GHANA HEALTH SERVICE ETHICAL REVIEW COMMITTEE}
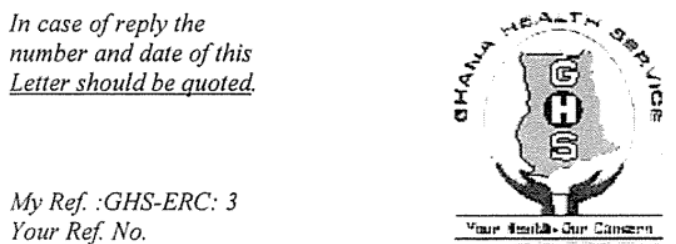

Research \& Development Division

Ghana Health Service

P. O. Box MB 190

Accra

Tel: $+233-302-681109$

Fax $+233-302-685424$

Email: Hannah.frimpong@ghsmail.org

$22^{\text {nd }}$ August, 2014

Andrews Adjei Druye

Victoria University of Wellington

New Zealand

\section{ETHICAL APPROVAL - ID NO: GHS-ERC: 07/07/14}

The Ghana Health Service Ethics Review Committee has reviewed and given approval for the implementation of your Study Protocol titled:

"Self management strategies for sickle cell disease in Ghana"

This approval requires that you inform the Ethical Review Committee (ERC) when the study begins and provide Mid-term reports of the study to the Ethical Review Committee (ERC) for continuous review. The ERC may observe or cause to be observed procedures and records of the study during and after implementation.

Please note that any modification without ERC approval is rendered invalid.

You are also required to report all serious adverse events related to this study to the ERC within seven days verbally and fourteen days in writing.

You are requested to submit a final report on the study to assure the ERC that the project was implemented as per approved protocol. You are also to inform the ERC and your sponsor before any publication of the research findings.

Please always quote the protocol identification number in all future correspondence in relation to this approved protocol

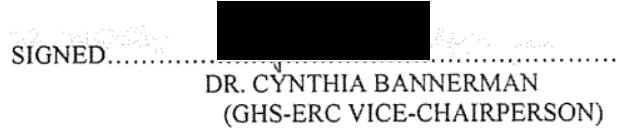

Cc: The Director, Research \& Development Division, Ghana Health Service, Accra 


\section{Appendix 3. Permission from Ashanti Regional Health Directorate}

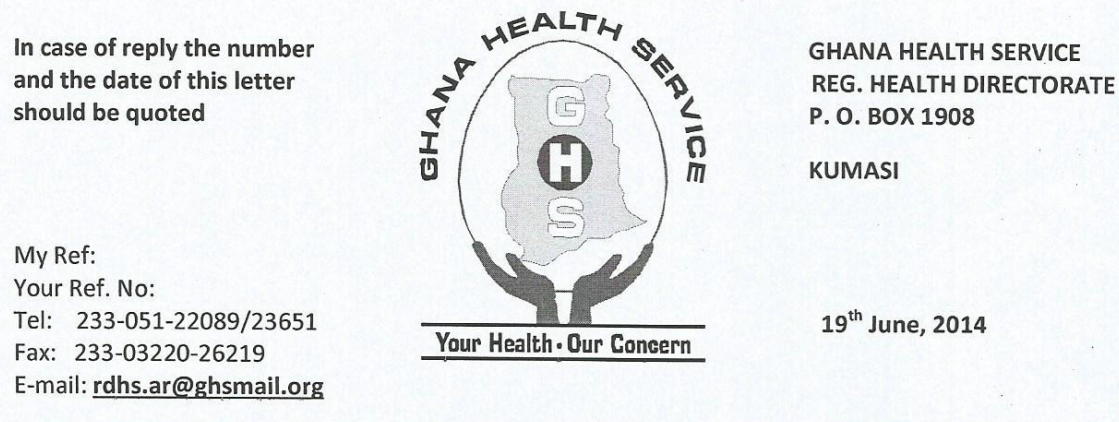

Andrews Adjei Druye

Victoria University in Wellington

New Zealand

\section{PERMISSION TO CONDUCT RESEARCH ON SELF MANAGEMENT STRATEGIES FOR SICKLE CELL DISEASE IN GHANA}

This serves to inform you that permission is offered for you to access our facilities to conduct the above research study in the Ashanti region.

We understand that you intend to collect self-reported data (focus groups, interviews and survey) among a cross section of health professionals, sickle cell patients and parents of children with sickle cell disease in the Ashanti region.

The Regional Health Directorate is satisfied that the research study you intend to undertake is commensurate with the activities of the Ghana Health Service.

You must ensure that you understand and comply with the requirements of the Ghana Health Service regarding research and cooperate with the health workers in discharging their duties.

While undertaking the research you will remain accountable to your university but you are required to collaborate with your field supervisor from the Ghana Health Service.

The Regional Health Directorate is to be provided with the study updates at the end of the data collection and a summary of the findings on completion of the study.

We wish you every success in this endeavour.

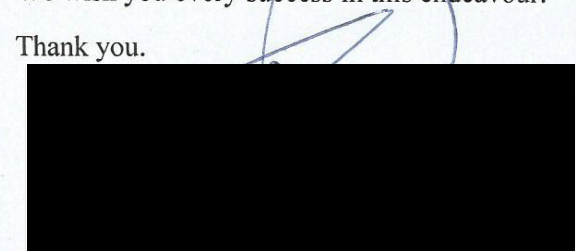

DR. ALEXIS NANG-BEIFUBAH

REGIONAL DIRECTOR OF HEALTH SERVICE

GHANA HEALTH SERVICE

KUMASÍ

$\circ$ 


\section{Appendix 4. Komfo Anokye Teaching Hospital Approval}

\section{KWAME NKRUMAH UNIVERSITY OF SCIENCE AND TECHNOLOGY}

COLLEGE OF HEALTH SCIENCES

SCHOOL OF MEDICAL SCIENCES / KOMFO ANOKYE TEACHING HOSPITAL COMMITTEE ON HUMAN RESEARCH, PUBLIGATION AND ETHIGS

Our Ref: CHRPE/AP/287/14

Mr. Andrews Adjei Druye

Graduate School of Nursing

Midwifery and Health

Post Office Box 7625

Victoria University of Wellington

NEIW ZEALAND.

Dear Sir,

\section{LETTER OF APPROVAI}

Protocol Title "Self Management Strategies for Sickle Cell Disease in Ghana".

Proposed Site:

The Sickle Cell Clinics of Komfo Anokye Teaching Hospital, Bekwai Government Hospital, Agrovesum District Hospital, Mampong Government Hospital, Obuasi Government Hospital, Agogo Hospital, Offinso Government Hospital and Nkenkenso District Hospital.

Sponsor:

Victoria University of Wellington.

Your submission to the Committee on Human Research, Publications and Ethics on the above named protocol refers.

The Committee reviewed the following documents:

- A notification letter of $4^{\text {th }}$ July, 2014 from the Komfo Anokye Teaching Hospital (study site) indicating approval for the conduct of the study in the Hospital.

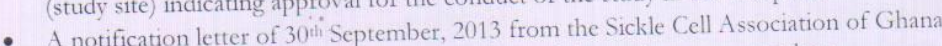
(study site) indicating approval for the conduct of the study among members.

(

(study site) indicating approval for the conduct of the study in the Region

- A completed CHRPE Application Form.

- Participant Information Leaflet and Consent Form.

- Research Proposal

- Questionnaire.

The Committee has considered the ethical merit of your submission and approved the protocol. The approval is for a fixed periof ethical approval at anytime if your study is found to contravene the approved protocol.

Data gathered for the study should be used for the approved purposes only. Permission should be sought from the Committec if any amendment to the protocol or use, other than submitted, is made of your research dat.

The Committee should be notified of the actual start date of the project and would expect a report on your study, annually or at the close of the project, whichever one comes first. It should also be informed of an publication arising from the study.

Room 7 Block J, School of Medical Sciences, KNUST, University Post Office, Kumasi, Ghan 


\section{Appendix 5. Komfo Anokye Teaching Hospital Approval 2}

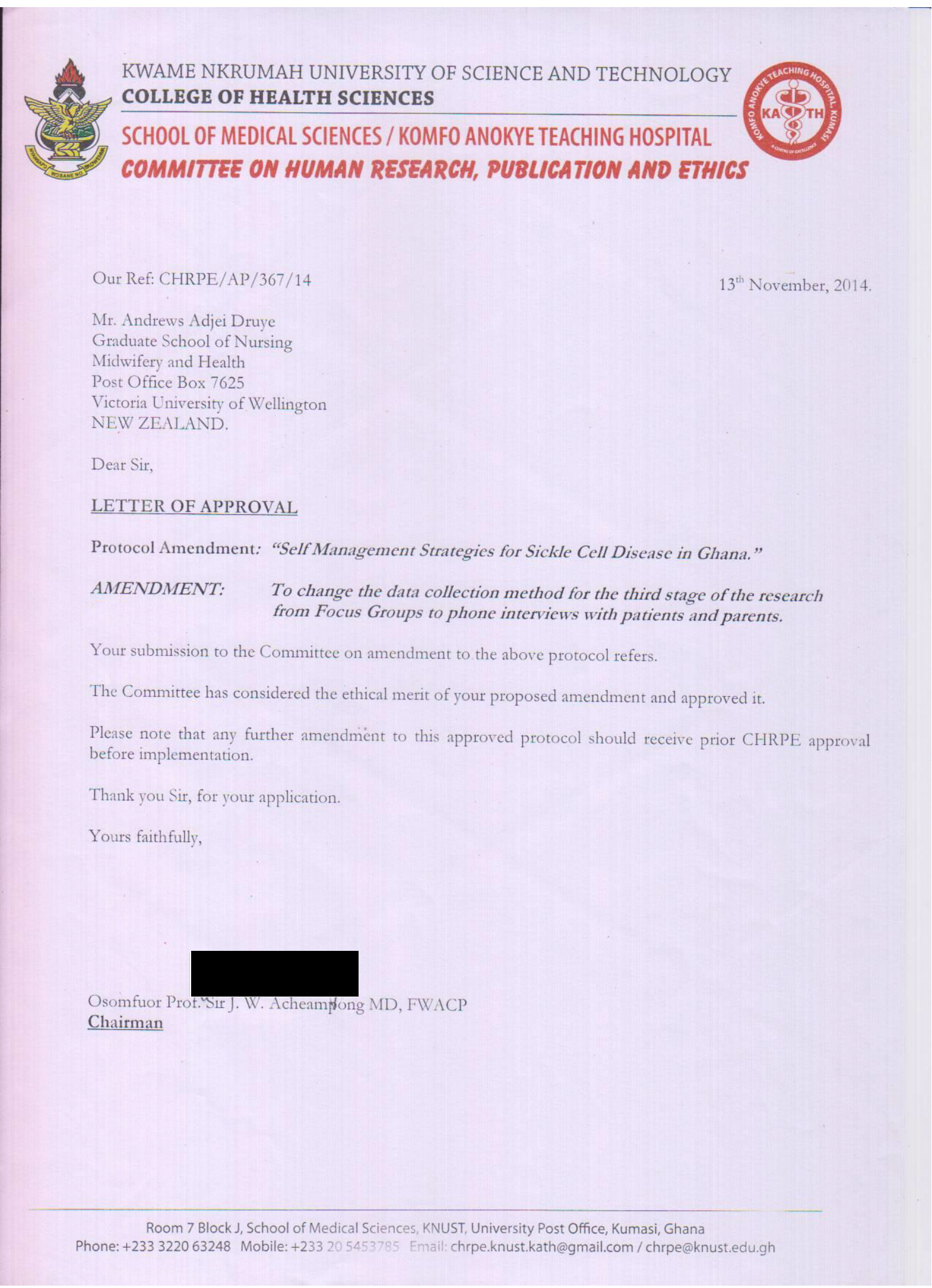




\section{Appendix 6. Approval from Komfo Anokye Teaching Hospital}

\section{KOMFO ANOKYE TEACHING HOSPITAL}

Directorate of Child Health

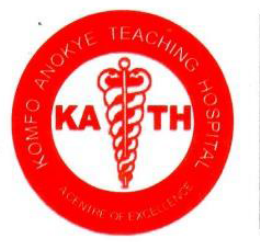

P. O. Box 1934

KUMASI - GHANA

Tel: +233 - 3220-22301 - 4

Fax: +233 - 3220 - 24654/24621

Website: www.kathhsp.org

Our Ref. No:

Your Ref. No:

The Deputy Director

Research and Development Unit

KAH

Dear Sir

LETTER OF SUPPORT: "SELF MANAGEMENT STRATEGIES FOR SICKLE CELL DISEASE IN GHANA AT THE KOMFO ANOKYE TEACHING HOSPITAL (KATH)"

I am writing in support of Andrews Adjei Druye, Principal Investigator, Graduate School of Nursing, Midwifery and Health, Victoria University of Wellington's application for registration and ethical clearance for the conduct of research on the above-mentioned topic

He has sought permission to carry out the research work as titled above in our Directorate.

We have taken a look at the protocol and we are in support and therefore give permission of the Directorate for him to seek ethical clearance and subsequently proceed to carry out the study. 


\title{
Appendix 7. Komfo Anokye Teaching Hospital Supervisor's Support Letter
}

\author{
KOMFO ANOKYE \\ TEACHING HOSPITAL
}

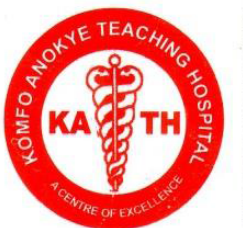

P. O. Box 1934 KUMASI - GHANA

Tel: + 233 - 3220-22301 - 4

Fax: +233 - $3220-24654 / 24621$

Website: www.kathhsp.org

Our Ref. No:

Your Ref. No:

The Chairman

$20^{\text {th }}$ June 2014

GHS-Ethical Review Committee

P.O.Box MB 190

Accra-Ghana

Dear Sir,

\section{SUPPORT LETTER FOR LOCAL SUPERVISION}

I have the pleasure to support the $\mathrm{PhD}$ research project; Self Management Strategies for Sickle Cell Disease in Ghana led by Mr Andrews Adjei Druye.

The aim of the proposed study is well aligned with my research interest as a clinician and researcher for sickle cell disease.

This research has the potential of contributing to an important aspect of sickle cell disease management which has received little scientific work in Ghana. I also see a strong potential to building knowledge and research in the field of self management for haemoglobinopathies whilst benefiting our national efforts in finding solutions and to lessen the burden of sickle cell disease on patients and families. I therefore support the study.

As the local supervisor, I will bring my long term experience of working with sickle cell patients and families as well as supervision of students' projects as a senior lecturer to bear in the proposed study.

I have had a previous working relationship with Mr Andrews Adjei Druye on the Newborn Screening for Sickle Cell Disease Research Project and I have no doubt in my mind that we can have a productive working relationship.

I hope that the proposed study receives a favourable review as I look forward to working with the Principal investigator and his New Zealand supervisors on this important project.

Thank you.

Yours sincerelv.

Dr. Alex Osei-Akoto

Senior Lecturer and Peadiatrician Doctor in

Charge of sickle cell clinic KATH 

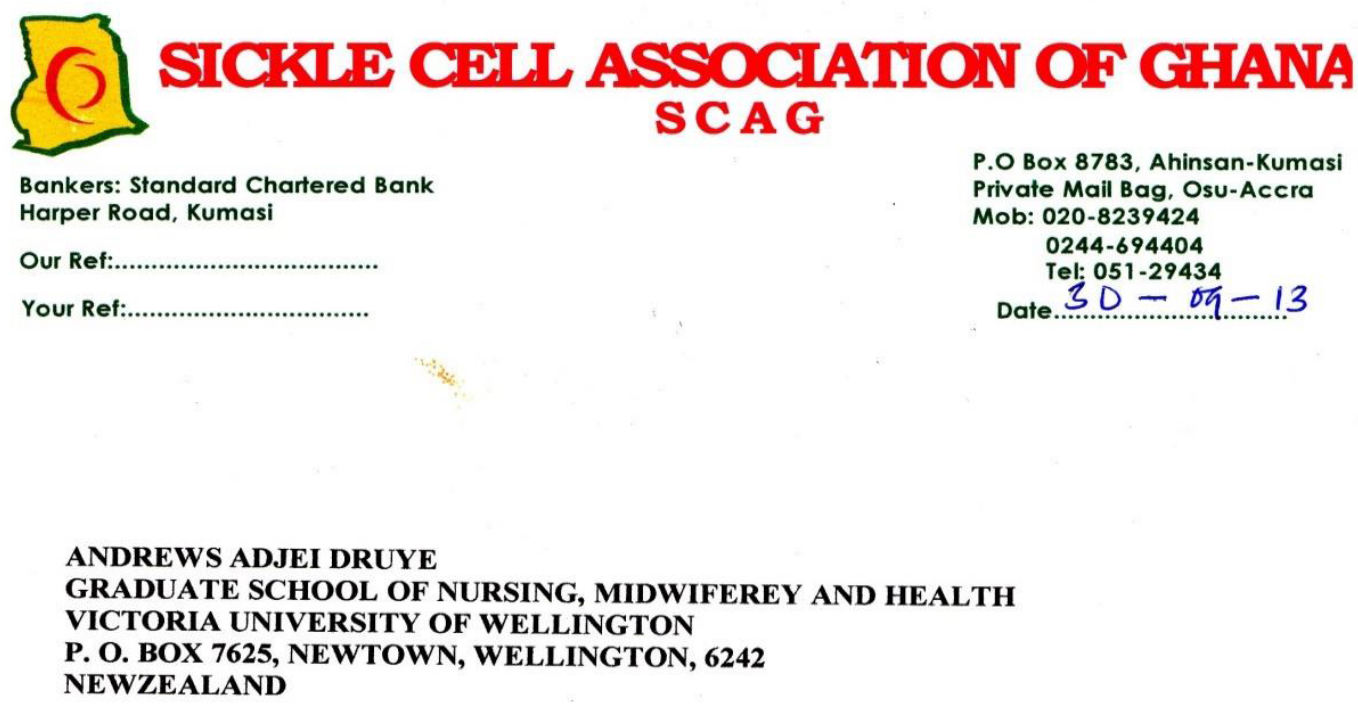

I write on behalf of the Sickle Cell Association of Ghana to indicate our acknowledgement of your request for permission to carry out a research study among Sickle Cell Patients and Parents.

We understand that you intend to study ''Self Management Strategies for Sickle Cell Disease in Ghana" and that you hope to interview a cross section of parents and patients in the country.

On behalf of the sickle cell association of Ghana, I write to offer you permission to carry out this study among our members.

Please feel free to contact my office for any further enquiries if necessary.

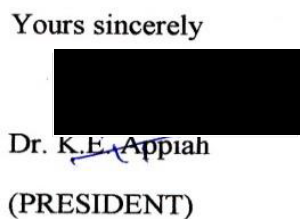




\section{Appendix 9. KATH Information Leaflet}

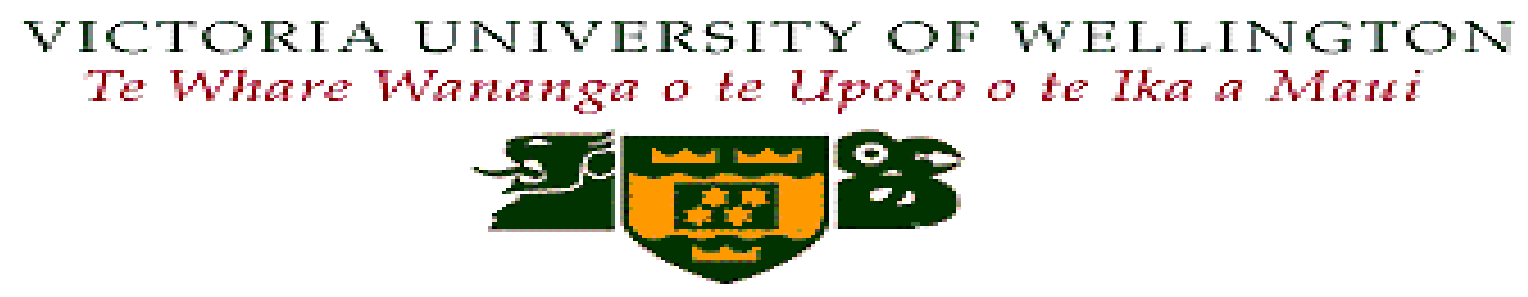

\section{Participant Information Leaflet}

\section{Self Management Strategies for Sickle Cell Disease in Ghana}

Hello. My name is Andrews Adjei Druye. I am a lecturer at the University of Cape Coast, Ghana, currently pursuing a doctoral study $(\mathrm{PhD})$ on a Victoria Doctoral Scholarship at the Graduate School of Nursing, Midwifery and Health at Victoria University of Wellington, New Zealand.

\section{Background}

I am conducting this research project to write a thesis for my doctoral degree at the Victoria University of Wellington, New Zealand.

\section{Purpose(s) of research:}

The purpose of this study is to collect information on self management strategies, which are the measures persons with sickle cell disease and parents of children with sickle cell disease use on a day-to-day basis, by themselves, to control and reduce the impact of this disease on their lives. I will also investigate how useful people find these measures.

\section{Procedure of the research and what shall be require of participant}

People who can participate in this study are:

- Persons with sickle cell disease 16 years and older

- Parents of children (15 years and under) with sickle cell disease

- Health professionals (doctors, nurses, health educators) who work with persons with sickle cell disease or their parents

The information that will be required of people who participate in this research includes:

- A description of the disease by patients and parents and how it affects lives

- What persons with sickle cell disease and families do to prevent illnesses and problems when patients are generally feeling well

- How people recognise that a person with sickle cell disease is sick or has had a problems related to the disease

- What people with sickle cell disease do for themselves or what parents do for their children when the person with sickle cell disease is sick or develops a problem

- How useful people find self management measures on their health and quality of life.

- What health professionals in Ghana recommend for self management for sickle cell disease. 
If you participate in this study, you will take part in a focus group, personal interview or asked to fill a questionnaire.

- Up to 10 health professionals will be interviewed via Skype.

- Up to 32 adults sickle cell patients and parents with children with sickle cell disease will be will be interviewed via telephone.

- Approximately 400 adults sickle cell patients and parents will be asked to complete a survey questionnaire.

The interview and focus groups discussions will be recorded with audiotapes. These sessions will take 30minutes to one hour. Before you begin, telephone or skype interview will be asked to sign a consent form or give verbal consent to show that they agree to participate in the study. Respondents to the survey will not be required to sign a consent form but their consent will be inferred if they complete and return the survey questionnaire.

\section{Risk(s):}

It is not likely that the process will cause any harm to you but in case you get distressed in the course of the interactions you can be excused to take a rest and continue later on the same day or another time.

\section{Benefit(s):}

If you participate in the study, it will be an opportunity for you to contribute your experiences on what people do for themselves in managing sickle cell disease. This information will in the future, help to design programme that will train and support persons with sickle cell disease and their families to effectively manage some of the day-to-day problems associated with the disease in ways that will be relevant to their lives at home.

\section{Confidentiality:}

After the data is collected, it will be processed and stored securely so that no other person has access to it apart from me or my supervisors. The information will be used purely for this research and will be destroyed after 10years. If you do not want your data to be included in the analysis, you must tell me within two weeks after the data collection. In such situation, all data about you will be removed and destroyed.

\section{Voluntariness:}

Your participation in this research is purely voluntary. You are not under any compulsion to participate. You can also decline to answer any question that might inconvenience you.

\section{Alternatives to participation:}

If you decide not to participate in this study; it will not affect your relationship with the staff of this hospital or your treatment services in any way.

\section{Withdrawal from the research:}

You are at liberty to withdraw your participation from the study any time you wish to do so without any form of penalty and without having to explain yourself. However, if you intend to withdraw from the focus groups I will appreciate if you could tell me before the discussion begins. 


\section{Consequence of Withdrawal}

If you decide to withdraw from the study after some information has been obtained from you, I will appreciate if tell me within two weeks from the day you provided the information. In this case, all data about you will be removed and destroyed. However, if you inform me of your withdrawal after two weeks, your information may have been added to that of others in the analysis and I will not be possible to remove yours since names and other identifying information will not be included in the analysis.

\section{Costs/Compensation}

You will not be paid for participating in this study. However, refreshment will be provided for survey participants. You are not likely to incur any cost for your participation in this study because survey participants will provide the information on the day you attend the sickle cell clinic or association meeting. However if anyone needs to return to the clinic to complete the research, a travel cost of up to 30 Ghana cedi's will be given to him by the principal investigator to cover travel expenditure.

\section{Contacts:}

If you require any further information or clarification please contact me or my supervisors, Dr. Katherine Nelson, Dr Brian Robinson, Dr Alex Osei-Akoto, Dr. Daniel Ansong. Contact details are provided below.

Approval for this study was obtained from the Human Ethics Committee of Victoria University of Wellington, New Zealand, as well as the Research and Development Units of the Ghana Health Service and the Komfo Anokye Teaching Hospital. Permission was also obtained from health and hospital authorities and the Executive Committee of the Sickle Cell Association of Ghana.

Thanking you in anticipation of your participation and cooperation.

Andrews Adjei Druye (Principal Investigator)

PhD student

Graduate School of Nursing, Midwifery and Health

Victoria University of Wellington

Wellington, NewZealand

E-mail: Andrews.druye@vuw.ac.nz

Tel (Ghana)

Tel (Newzealand):

Dr. Katherine Nelson (Research Supervisor)

Senior Lecturer

Graduate School of Nursing, Midwifery and Health

Victoria University of Wellington

Wellington, Newzealand

E-mail: Kathy.nelson@vuw.ac.nz

Cell phone:

Office: 00644636138 
Dr. Brian Robinson (Research Supervisor)

Senior Lecturer

Graduate School of Nursing, Midwifery and Health

Victoria University of Wellington

Wellington, Newzealand

E-mail: Brian.Robins@vuw.ac.nz

Cell phone:

Office: 00644636155

Dr. Alex Osei-Akoto (Local Supervisor)

Senior Lecturere

Kwame Nkrumah University of Science and Technology

School of Medical Sciences

Kumasi, Ghana

E-mail:

Cell phone:

Office: 00233322036751

Dr. Daniel Ansong

Senior Lecturere

Kwame Nkrumah University of Science and Technology

School of Medical Sciences

Kumasi, Ghana

E-mail:

Cell phone:

Office: 0023332194995

Further, if you have any concern about the conduct of this study, your welfare or your rights as a research participant, you may contact:

The Chairman

Committee on Human Research and Publication Ethics

Kwame Nkrumah University of Science and Technology

Kumasi

Tel: 22301-4 ext 1098 or 
VICTORIA UNIVERSITY OF WELLINGTON

Te Whave Wananga o te Upoko o te Ika a Mani

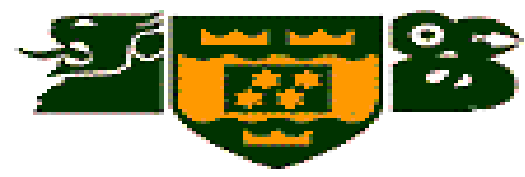

\section{SELF MANAGEMENT STRATEGIES FOR SICKLE CELL DISEASE IN GHANA}

\section{Consent Form for Health Professionals Interview}

\section{A. Statement and Signature of Person Giving Consent}

- I declare that I have received adequate information and explanation of the purpose and content of the study to investigate self management strategies for sickle cell disease in Ghana by the researcher.

- I understand that my participation is voluntary and that I can withdraw from the study at any time without having to explain myself

- I understand that any information I provide will be held in strict confidence. This implies that any data collected from me will not be exposed to any person apart from the researcher and the supervisors.

- I have also been informed that the recordings will be electronically wiped out after the research.

- I understand that the results of this study will be presented to Victoria University of Wellington and that I can get access to a copy of the report through a link that will be provided to the Sickle Cell Association of Ghana.

- I understand that journal articles and conference presentations may be extracted from the findings of the study.

- I have had adequate information about whom to contact if I require further information about the research and my participation.

- I have received a copy of the information leaflet and consent form to keep for myself I agree to take part in this research.

Name of Participant

Signature

Date:

\section{B. Investigator's Statement and Signature}

I have fully explained this research to and have given sufficient information including the risks and benefits to enable him/her make informed decision to or not to participate.

DATE

NAME 


\section{Appendix 10. Parental Consent Forms for Children Under 18 Years}

VICTORIA UNIVERSITY OF WELLINGTON

Te Whare Wananga o te Upoko o te Ika a Mani

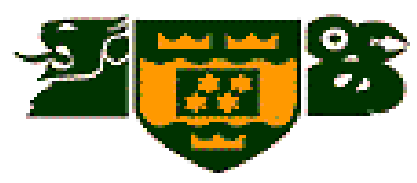

\section{Parental Consent Form for Interview Participants Under 18 Years}

\section{Self Management Strategies for Sickle Cell Disease in the Ashanti Region of Ghana}

\section{A. Statement and Signature of Person Giving Consent}

- I declare that I have received adequate information and explanation of the purpose and content of the study to investigate self management strategies for sickle cell disease in Ghana by the researcher.

- I understand that my child's participation is voluntary and that he/she can withdraw their participating from the interview without having to explain him/herself.

- I understand that any information my child provides will be held in strict confidence. This implies that any data collected from my child will not be exposed to any person apart from the researcher and the supervisor.

- I have also been informed that the recordings will be electronically wiped out after the research.

- I understand that the results of this study will be presented to Victoria University of Wellington and that I can get access to a copy of the report through a link that will be provided to the Sickle Cell Association of Ghana.

- I also know that journal articles and conference presentations may be extracted from the findings of the study.

- I have had adequate information about whom to contact if I require further information about the research and my child's participation.

I agree that my child............................... can participate in this research study.

Name

Mother/Father/ Guardian (underline the one applicable)

Date: Signature/Thumbprint

Witness' signature (If the person giving the consent could not read for themselves) 
Witness'

name.

\section{B. Investigator's Statement and Signature}

I have fully explained this research to and have

given sufficient information including the risks and benefits to enable him/her make informed decision to or not to allow his/her

child.

to participate.

DATE

NAME

You may contact the Administrator of the Ethical Review Committee of the Ghana Health Service for further clarification regarding ethical approval for this study.

Name: Hannah Frimpong

Telephone: or 


\section{Appendix 11. GHS Consent Above 18 years}

VICTORIA UNIVERSITY OF WELLINGTON

Te Whare Wananga o te Upoko o te Ika a Mani

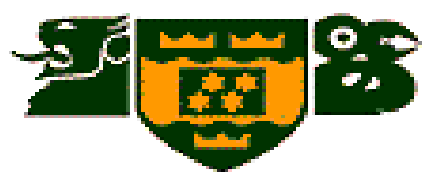

\section{Consent Form for Interview Participants Above 18 Years}

\section{Self Management Strategies for Sickle Cell Disease in the Ashanti Region of Ghana}

\section{B. Statement and Signature of Person Giving Consent}

- I declare that I have received adequate information and explanation of the purpose and content of the study to investigate self management strategies for sickle cell disease in the Ashanti of Ghana by the researcher.

- I understand that my participation is voluntary and that I can withdraw my participating from the interview at any time without having to explain myself.

- I understand that any information I provide will be held in strict confidence. This implies that any data collected from me will not be exposed to any person apart from the researcher and the supervisor.

- I have also been informed that the recordings will be electronically wiped out after the research.

- I understand that the results of this study will be presented to Victoria University of Wellington and that I can get access to a copy of the report through a link that will be provided to the Sickle Cell Association of Ghana.

- I also know that journal articles and conference presentations may be extracted from the findings of the study.

- I have had adequate information about whom to contact if I require further information about the research and my participation.

I agree to take part in this research.

Name of Participant

Signature

Date: Signature/Thumbprint

Witness' signature (If the person giving the consent could not read for themselves). 
Witness'

name.

B. Investigator's Statement and Signature

I have fully explained this research to and have

given sufficient information including the risks and benefits to enable him/her make informed decision to or not to participate.

DATE

NAME

You may contact the Administrator of the Ethical Review Committee of the Ghana Health Service for further clarification regarding ethical approval for this study.

Name: Hannah Frimpong

Telephone:

$$
\text { or }
$$




\section{Appendix 12. Health Professional's Interview Guide}

\section{VICTORIA UNIVERSITY OF WELLINGTON \\ Te Whare Wananga o te Upoko o te Ika a Maui

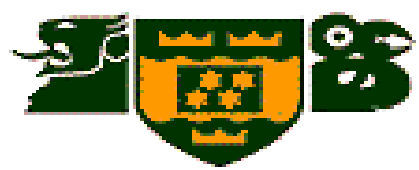

\section{Health Professionals Interview Guide}

\section{Self-Management Strategies for Sickle Cell Disease in the Ashanti Region of Ghana}

Name of health professional

Professional background

Number of years of practice as health professional

Number of years of experience with SCD.

Health facility

1. What do you tell your clients and families to do in caring for SCD at home

2. In your experience what self management actions do patients with sickle cell disease or parents with children with sickle cell disease usually take to:

- keep themselves or their children well

- manage illnesses or complications related to the disease

3. In which of the sickle cell related conditions do patients and families usually use self management measures?

4. What self management measures do you recommend for patients and parents when patients are:

- in stable condition (adults and children)

- have an illness or complication (adults and children)

5. How useful do you think self management measures could be for people with sickle cell disease

6. Are there any further self management measures you would like to share? 


\section{Appendix 13. Parents' Interview Guide}

\section{VICTORIA UNIVERSITY OF WELLINGTON \\ Te Whare Wananga o te Upoko o te Ika a Mani}

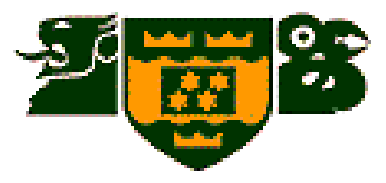

Interview Guide for Parents (with Twi Translation)

Self Management Strategies for Sickle Cell Disease in the Ashanti Region of Ghana

Ankorakora anamontuo fa ahotutuo yare $\varepsilon$ ho wo Asantemantem mu a عw

\section{Ghana.}

1. Tell us about your child with sickle cell diseases: (The items in the box will be given to people to fill)

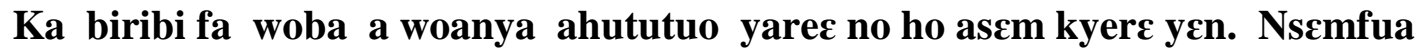

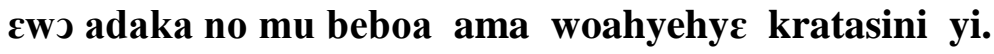

- Age (parent/child)

Mfie a woadi

- Health insurance status

Apomoden nsiakyibaa gyinapen

- Income (direct/ source of income)

Sika a wonya

- Availability of sickle cell clinic in area of residence

Ahotutuo ayaresabea ben wopatem ho

- Closeness of any hospital or clinic to the clients

Ayaresabea kese $\varepsilon$ anaa ketewa bi ben syarefos no.

- Ease of access to clinical care/SCD Clinic

- Education

Wo nwomanim te sen 
- Employment

Wo ye adwuma ben

- Type of sickle cell disease of your child

Sen na wo ba ahotutuo yare no tee.

- Relationship/marital status

- Nunber of children with SCD

\section{GENERAL}

2. What do you do on a daily basis to ensure that your child with SCD is healthy(stable condition)

3. Describe what you normally do by yourself to reduce or prevent illness or problems on days when your child is generally feeling well.

\section{SELF MONITORING}

4. What do you do on daily basis by yourself to check the progress of your child's health?

5. What observations do you do on a daily basis on your child with SCD

Dee na wo ye dabiara de hunu wo ba no apomuden nkosos?

\section{SELF DIAGNOSIS}

6. How do you recognise that your child has an illness or complication related to sickle cell disease?

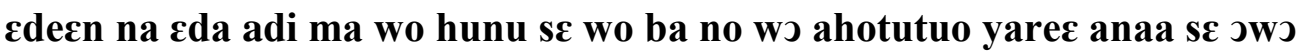
haw bi a ete $s \varepsilon$ ahotutuo yarec.

\section{SELF TREATMENT}

7. What you do you normally do by yourself at home when your child with SCD has a crisis

8. In the past six months what sickle cell related sickness or problem of your child has been of most concern to you?

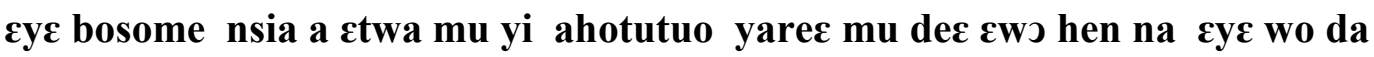
dwene paa fa wo ba no ho.

9. What did you by yourself to deal with the problem of most concern that affected your child? 


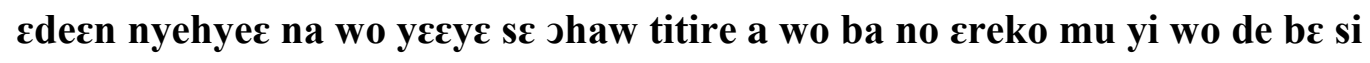
ano.

10. What other sickle cell related illnesses have your child ever suffered in the past

11. What are some of the things you did to manage these illnesess(based on specific illnesses)

12. What else have you ever done by yourself to manage other sickle cell disease related illness or problems in the past?

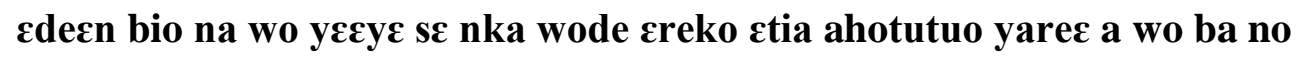

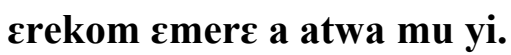

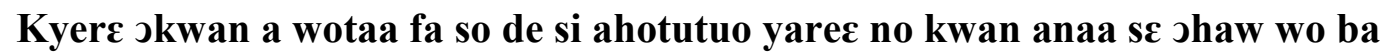
no kom ebere Jte apo.

13. How helpful do you find the measures you take by yourself to manage sickle cell disease related problems for your child?

Sen na wo si hunu emoa wode agu kwam de reboa wo ba ahotutuo yaree no?

14. What else do you know parents with sickle cell disease do by themselves to keep their children well but you have not tried before?

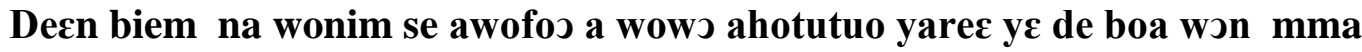
nanso wo ntuu ho anamon da.

15. What else will you want to try but cannot?

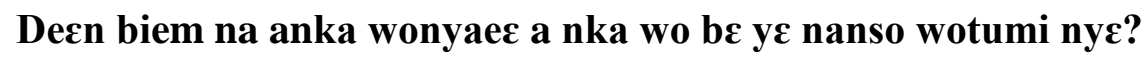

\section{OVERARCHING QUESTIONS}

16. What do you usually try to avoid

17. What do you usually ensure that you do very well

18. What cations or precautions 


\section{Appendix 14. Parents' Questionnaire}

Questionnaire ID

Clinic Name.

Date.

Place of Residence

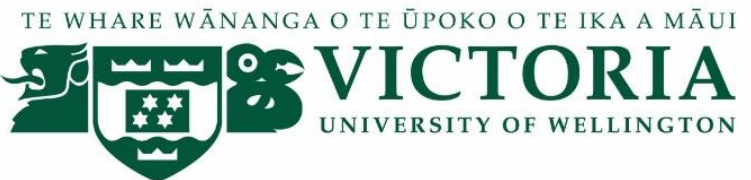

\section{SELF MANAGEMENT STRATEGIES FOR SICKLE CELL DISEASE IN GHANA}

Dear respondent. Thank you for agreeing to participate in this survey. This study is intended to investigate what people do for themselves to manage SCD at home and communities. The survey is organised into eight sections (background information; preventive health and maintenance actions; self monitoring of disease; self diagnosis; self treatment; self management resources; vulnerability and contextual factors; health outcomes. The survey takes approximately 30 minutes to complete. All responses to the survey will be reported in aggregate format. While quotations will be used in the reporting of survey findings, every effort will be made to present these in a non-identifiable way. Consent is assumed by completion and return of the survey. There is no need to sign the survey or identify yourself by name in the survey.

1. What is the age of your child in years?.

2. Is your child: Male

Female

3. What is the highest year of schooling you completed? (Please circle one)

a) None

b) Primary

c) Junior High/ Middle School

d) Senior High/Secondary

e) Tertiary

f) Others (please specify)

4. What type of Sickle cell disease does your child have?(circle one)
a) $\mathrm{HbSS}$
b) $\mathrm{HbSC}$
c) Hb SBetha plus thalassaemia
d) $\mathrm{Hb} \mathrm{S}$ Betha zero thalacaemia
e) Don't Know
f) Other (Specify) 
5. How old was your child when he/she was first diagnosed with sickle cell disease? (Years / months / weeks)

\section{PREVENTIVE HEALTH AND MAINTENANCE CARE}

This section asks about things you do or precautions you take to stay healthy and reduce illnesses.

6. How often do you normally do the following to keep your child well or prevent illness? Circle one number for each item to show how often you have done the activity.

\begin{tabular}{|c|c|c|c|c|c|c|c|c|}
\hline a) & Take folic acid & 0 & 1 & 2 & 3 & 4 & $\begin{array}{c}\text { or more } \\
5\end{array}$ & $\begin{array}{l}\text { necessary } \\
6\end{array}$ \\
\hline b) & Take vitamin supplements & 0 & 1 & 2 & 3 & 4 & 5 & 6 \\
\hline c) & Takes blood tonics & 0 & 1 & 2 & 3 & 4 & 5 & 6 \\
\hline d) & Takes malaria prophylaxis & 0 & 1 & 2 & 3 & 4 & 5 & 6 \\
\hline e) & Take worm medication & 0 & 1 & 2 & 3 & 4 & 5 & 6 \\
\hline f) & Take herbs or traditional & 0 & 1 & 2 & 3 & 4 & 5 & 6 \\
\hline g) & Drink water/fluids & 0 & 1 & 2 & 3 & 4 & 5 & 6 \\
\hline h) & Follow a special diet & 0 & 1 & 2 & 3 & 4 & 5 & 6 \\
\hline i) & Avoid strenuous activities & 0 & 1 & 2 & 2 & 4 & 5 & 6 \\
\hline j) & Dress to stay warm & 0 & 1 & 2 & 3 & 4 & 5 & 6 \\
\hline k) & Avoid cold drinks/water & 0 & 1 & 2 & 3 & 4 & 5 & 6 \\
\hline 1) & Avoid cold baths & 0 & 1 & 2 & 3 & 4 & 5 & 6 \\
\hline m) & Avoid cold places & 0 & 1 & 2 & 3 & 4 & 5 & 6 \\
\hline n) & Sleeps in mosquito net & 0 & 1 & 2 & 3 & 4 & 5 & 6 \\
\hline o) & Use mosquitoes spray/coil & 0 & 1 & 2 & 3 & 4 & 5 & 6 \\
\hline p) & Avoid thinking about disease & 0 & 1 & 2 & 3 & 4 & 5 & 6 \\
\hline q) & Attend clinical appointments & 0 & 1 & 2 & 3 & 4 & 5 & 6 \\
\hline r) & Keeps vaccination up to date & 0 & 1 & 2 & 3 & 4 & 5 & 6 \\
\hline
\end{tabular}

s) Other (Specify 


\section{SELF MONITORING}

In this section, I will like to find out what you normally do on a daily basis to check the progress of your health

7. Some people say they check their health every day. For example some people inspect their eyes for paleness each day. What do you usually do on a daily basis on your child?

(Circle YES or NO to as many as applied)
a) Check urine colour
b) Check for jaundice (yellow eyes and body)
c) Look for palenes
d) Check temperature
e) Palpate the abdomen
f) Look for signs of diarrhoea and vomiting
g) Observes general behaviour
h) Monitor growth
i) Observes for pain
j) Keeps a diary or records
k) Others (please specify)

Yes

No

Yes

No

Yes

No

Yes

No

Yes

No

Yes

No

Yes

Yes

No

Yes

No

Yes

No

No

Yes $\quad$ No

\section{SELF DIAGNOSIS}

In this section, I will like to find out how you usually know that you have a crisis or problems from $S C D$.

8. How many times did your child have a painful crisis in the past 12 months?.....

9. What were the things that causes your child to get sick or have painful crisis? 
10. How do you usually know that your child has a painful crisis?

11. Apart from the experience of painful events, which of the following signs and symptoms do you get when your child has sickle cell related problem? ( Circle YES or NO to all that applied)

a) Urine colour changes

b) Yellow eyes and body

Yes No

c) High hotness of the body

d) Dizziness/ collapse

e) Diarrhoea

f) Vomiting

g) Paleness of the eyes and body

Yes

No

Yes

No

Yes No

Yes No

No

h) Swollen joints

No

i) Abdominal pains

Yes

No

j) Difficulty in breathing

Yes

No

k) Painful erection of the penis (males)

Yes

No

1) Swollen hands and feet

Yes

No

m) Prolong crying of children

Yes

No

n) Other (please specify)

Yes

No

Yes

No

\section{SELF TREATMENT}

In this section, I will like to know what you do to care for yourself when you have problems or illness from $S C D$

12. Has your child experienced painful episode or crisis in the past 12 months? ( Yes / No)

12a. If YES when was the last time he/she experienced painful crisis?

(If No go to Q13)

$12 b$. What did you do by yourself to manage the pain crisis? 
12c. please circle how helpful you found what you did for your child to manage the painful crisis
a) Very helpful
(ebua me paa)
b) Somehow helpful
(ebua me kakra)
c) Neither helpful nor unhelpful (eho ne ho)
d) Not so helpful (amoa me papa)
e) Not helpful at all
(amoa me kuraa)
f) Not applicable

Has your child experienced very high hotness of the body (aho hye) in the past 12 months?

(Yes /

No)

13a. If YES when was the last time he/she experienced fever? ... If No go to Q14)

13b. What did you do by yourself to manage the fever?

13c. Please circle how helpful you found what you did by yourself to manage the fever
a) Very helpful
(ebua me paa)
b) Somehow helpful
(ebua me kakra)
c) Neither helpful nor unhelpful (eho ne ho)
d) Not so helpful
(amoa me papa)
e) Not helpful at all
(amoa me kuraa)
f) Not applicable

13. Has your child experienced severe headache in the past 12 months?

(Yes / No)

14a. If YES when was the last time he/she experienced headache? (If No go to Q15)

14b. What did you do by yourself to manage the headache? 
14c. Please circle how helpful you found what you did for your child to manage the severe headache
a) Very helpful
(ebua me paa)
b) Somehow helpful
(ebua me kakra)
c) Neither helpful nor unhelpful (eho ne ho)
d) Not so helpful
(amoa me papa)
e) Not helpful at all
(amoa me kuraa)
f) Not applicable

14. Has your child experienced sudden weakness or loss of feeling (titiritii) and movement in the past 12 months?

(Yes / No)

15a. If YES when was the last time he/she experienced sudden weakness or loss of feeling?

(If NO go Q16)

15b. What did you do by yourself to manage the weakness and loss offeeling?

15c. Please circle how helpful you found what you did for your child to manage weakness or loss of feeling?
a) Very helpful
(ebua me paa)
b) Somehow helpful
(ebua me kakra)
c) Neither helpful nor unhelpful (eho ne ho)
d) Not so helpful
(amoa me papa)
e) Not helpful at all
(amoa me kuraa)
f) Not applicable

15. Has your child experienced leg ulcers (sore /wound) in the past 12months? (Yes / No)

16a. If $\mathbf{Y E S}$ when was the last time your child experienced leg ulcers?

(If No go to $Q 17)$

16b. What did you do by yourself to manage the leg ulcers? 
16c. Please circle how helpful you found what you did by yourself to manage the leg ulcers
a) Very helpful
(ebua me paa)
b) Somehow helpful
(ebua me kakra)
c) Neither helpful nor unhelpful (eho ne ho)
d) Not so helpful
(amoa me papa)
e) Not helpful at all
(amoa me kuraa)
f) Not applicable

16. Has your child experienced painful erection of the penis (if male) in the past 12 months

(Yes / No)

17a. If $\boldsymbol{Y E S}$ when was the last time hel/she experienced painful erection of the penis?

No go to Q18)

17b. What did you do by yourself to manage the painful erection of the penis?

17c. Please circle how helpful you find what you did by yourself to manage the painful erection of the penis
a) Very helpful
(ebua me paa)
b) Somehow helpful
(ebua me kakra)
c) Neither helpful nor unhelpful (eho ne ho)
d) Not so helpful
(amoa me papa)
e) Not helpful at all
(amoa me kuraa)
f) Not applicable

17. Has your child experienced yellowing of eyes and body in the past 12 months (Jaundice)

(Yes I No)

18a. If $\mathbf{Y E S}$ when was the last time your child experienced jaundice?

(If No go to Q19)

18b. What did you do by yourself to manage the jaundice? 
18c. Please circle how helpful you found what you did by yourself to manage the jaundice
a) Very helpful
(ebua me paa)
b) Somehow helpful
(ebua me kakra)
c) Neither helpful nor unhelpful (eho ne ho)
d) Not so helpful
(amoa me papa)
e) Not helpful at all
(amoa me kuraa)
f) Not applicable

18. Has your child experienced shortage of blood (Paleness of eyes and body) in the past 12 months

(Yes / No)

19a. If YES when was the last time your child experienced shortage of blood?

(If No go to 20)

19b. What did you do by yourself to manage the shortage of blood?

19c. Please circle how helpful you found what you did by yourself to manage the shortage of blood
a) Very helpful
(ebua me paa)
b) Somehow helpful
(ebua me kakra)
c) Neither helpful nor unhelpful (eho ne ho)
d) Not so helpful
(amoa me papa)
e) Not helpful at all
(amoa me kuraa)
f) Not applicable

19. Has your child experienced abdominal (belly) swelling in the past 12 months? (Yes / No) 20a.If YES when was the last time he/she experienced belly swelling?

(If No go to Q18)

20b. What did you do by yourself to manage the belly swelling? 
20c. Please circle how helpful you found what you did for yourself to manage the belly swelling
a) Very helpful
(ebua me paa)
b) Somehow helpful
(ebua me kakra)
c) Neither helpful nor unhelpful (eho ne ho)
d) Not so helpful (amoa me papa)
e) Not helpful at all
(amoa me kuraa)
f) Not applicable

20. Has your child experienced hip problems in the past 12 months

(Yes/No)

21a. If YES when was the last time he/she experienced the hip problems?

(If No go to Q19)

21b. What did you do by yourself to manage the hip problems?

21c. Please circle how helpful you found what you did by yourself to manage the hip problem
a) Very helpful
(ebua me paa)
b) Somehow helpful
(ebua me kakra)
c) Neither helpful nor unhelpful (eho ne ho)
d) Not so helpful
(amoa me papa)
e) Not helpful at all
(amoa me kuraa)
f) Not applicable

21. Has your child experienced sudden seizures or convulsion(fit) in the past 12 months (Yes / No)

22a. If YES when was the last time he/she experienced seizures or convulsion?

(If No go to 20)

22b. What did you do by yourself to manage the seizures or convulsion? 
22c. Please circle how helpful you found what you did by yourself to manage the seizures or convulsion
a) Very helpful
(ebua me paa)
b) Somehow helpful
(ebua me kakra)
c) Neither helpful nor unhelpful (eho ne ho)
d) Not so helpful
(amoa me papa)
e) Not helpful at all
(amoa me kuraa)
f) Not applicable

22. Apart from what I have asked you, which other health problem (s) did your child have in the past 12 months?

$23 a$. What did you do by yourself to manage the problem(s)?

23b. How helpful did you find what you did for your child?

23. How often in the last 12 months did you do the following things to care for your child when he/she was sick? (Tick all that applies).

$\begin{array}{lccccc} & \begin{array}{c}\text { Not at } \\ \text { all }\end{array} & \begin{array}{c}\text { Only } \\ \text { once }\end{array} & \begin{array}{c}\text { Some } \\ \text { time }\end{array} & \begin{array}{c}\text { Most of } \\ \text { the } \\ \text { time }\end{array} & \begin{array}{c}\text { All the } \\ \text { time }\end{array} \\ \text { a) Gave pain medication( e.g. Para, Brufen) } & 0 & 1 & 2 & 3 & 4 \\ \text { b) Gave lot of fluids } & 0 & 1 & 2 & 3 & 4 \\ \text { c) Gave traditional medicine } & 0 & 1 & 2 & 3 & 4 \\ \text { d) Applied heat to affected part } & 0 & 1 & 2 & 3 & 4 \\ \text { e) Applied cold to affected part } & 0 & 1 & 2 & 3 & 4 \\ \text { f) Performed wound dressing } & 0 & 1 & 2 & 3 & 4 \\ \text { g) Used massage } & 0 & 1 & 2 & 3 & 4 \\ \text { h) Gave alcohol } & 0 & 1 & 2 & 3 & 4 \\ \text { i) Gave Indian hemp (wee) } & 0 & 1 & 2 & 3 & 4 \\ \text { j) Asked child to go to bed early } & 0 & 1 & 2 & 3 & 4 \\ \text { k) Tied affected limb } & 0 & 1 & 2 & 3 & 4 \\ \text { 1) Applied weight to affected limb } & 0 & 1 & 3 & 3 & 4\end{array}$


m) Said prayers

n) Asked child to reduced activities

o) Applied topical cream(e.g. Alcohol)

0

2

3

p) Used exercise or activities

0

1

2

\section{3}

\section{4}

q) Consulted a spiritualist(e.g. Pastor,Mallam)

0

2

3

4

r) Consulted a doctor/hospital

$\begin{array}{lllll}0 & 1 & 2 & 3 & 4 \\ 0 & 1 & 2 & 3 & 4\end{array}$

24. Have you ever used traditional medicine to manage your child's SCD?

(Yes / No)

(If No go to Q26)

If YES name the medicine, the problem used for and how you used it

\begin{tabular}{|l|l|l|}
\hline Name of medicine & Problems used for & $\begin{array}{l}\text { How used (e.g. swallowed, } \\
\text { applied on skin, enema) }\end{array}$ \\
\hline 1. & & \\
\hline 2. & & \\
\hline 3. & & \\
\hline 4. & & \\
\hline 5. & & \\
\hline 6. & & \\
\hline
\end{tabular}

25. Have you ever used conventional medicines (abrofo duro) to manage your child's SCD?

If YES name the medicine, the problem used for and how you used it

\begin{tabular}{|l|l|l|}
\hline Name of medicine & Problems used for & $\begin{array}{l}\text { How used (e.g. swallowed, } \\
\text { applied on skin, enema) }\end{array}$ \\
\hline 1. & & \\
\hline 2. & & \\
\hline 3. & & \\
\hline 4. & & \\
\hline 5. & & \\
\hline 6. & & \\
\hline
\end{tabular}

\section{SELF MANAGEMENT RESOURCES}

This section concerns the things you have in the house that you use to manage your SCD, how much you know about caring for SCD at home, and the support you get from other people in managing your SCD. 
26. Which of the following resources do you have in the house to help you to manage your child's SCD? (Please circle YES or No to all the option below)

a) Pain medication (e.g. Para)

Yes

Yes

No

b) Folic acid

Yes

No

c) Topical cream

Yes

No

d) Thermometer

Yes

No

e) Warm clothing

Yes

No

f) Wound dressing equipment

Yes

No

g) Dairy or record book

Yes

No

h) Health education materials eg posters

Yes

No

i) Hot water bottle

Yes

No

j) Other (please specify)

No

27. Tick all sources where you get information on SCD care at home
a) Health workers
b) Relatives
Yes
No
c) Friends
Yes
No
d) Radio
Yes
No
e) Television
Yes
No
f) Internet
Yes
No
g) Other (please specify)
Yes
No
Yes
No

28. Do you attend Sickle Cell Association meetings?

(Yes/No)

29. How would you rate your knowledge about caring for your child's SCD at home (Please circle one)
a) Excellent (me nim paa)
b) Very good (me nim kakra)
c) Good (me nim)
d) Fair (3ho ne ho)
e) Poor (me nnim koraa)

30. Have you ever attended any course, teaching session or read anything about sickle cell care at home? (Yes/No)

(If No go to 33)

31. If YES what was it? 
32. Who mainly helps you in caring for your child's SCD at home? (please circle one)
a) None
b) Parents
c) Relatives
d) Government office e.g. social welfare
e) NGO e.g. sickle cell association
f) Other (Specify).

33. How would you rate the level of support you get for the care of your child's SCD at home
a) Excellent
(3y3 papaapa)
b) Very good (3y3 paa)
c) Good
$(3 \mathrm{y} 3)$
d) Fair
(3y3 kakra)
e) Poor
(3ny3 koraa)

\section{HEALH OUTCOMES}

In this section, I will like to find out how you view your state of health and your quality of life

34. In general how would you say your child's health is (wo apom den ete sen)? (Please circle one)
a) Excellent
(3y3 papaapa)
b) Very good
(3у3 раa)
c) Good
(3y3)
d) Fair
(3y3 kakra)
e) Poor
(3ny3 koraa)

35. How would you rate you're your child's overall quality of life? (ase tena mu ete sen ma wo ). Please circle the number below that describes your child's overall quality of life in the past 12 months

$\begin{array}{ccccccccccc}0 & 1 & 2 & 3 & 4 & 5 & 6 & 7 & 8 & 9 & 10 \\ \text { Very poor quality }\end{array}$




\section{SOCIODEMOGRAPHIC AND HEALTH SERVICES ISSUES}

In this section I will like to get some background information about you, as well as how access sickle cell clinics and other health services to manage your SCD

36. What is your relationship to this child with SCD?
a) Mother
b) Father
c) Relative
d) Guardian
e) Others (Please specify)

37. What is your occupation? (Please circle one)
a) Artisan
b) Business/Trading
c) Government employer
d) Private salary worker
e) Retired
f) Sick leave
g) Unemployed
h) Parent
i) Student
j) Other (please specify)

38. What is your marital status? (Please circle one)
a) Single
b) Married
c) Divorced
d) Widowed
e) In relationship

39. Do you attend sickle cell clinic?

40. If YES to question 40, how many times have you attended the clinic in the past 12 months?

41. What other services health services do you attend for your child's SCD care? (E.g. mainstream hospitals, herbal clinic, homeopathic clinic etc.)

42. Approximately how many hours/minutes does it take you to travel from your home to the clinic you attend?
a) Less than 30 minutes
b) 30-60minutes
c) 1-3 hours
d) 4-7 hours
e) More than 7 hours
f) Not applicable 
43. Approximately how much income do you earn in a year in Ghana cedis in a month? (Go to $Q 36$ if you don't receive any income)
a) Less than 100
b) $100-499$
c) $500-899$
d) $900-1299$
e) $1300-1699$
f) $1700-2000$
g) More than 2000
h) None

44. What proportion of your income do you usually spend monthly on managing your child's SCD?
a) Just a little
b) Almost half
c) More than half
d) Almost all
e) All of my income
f) Don't know
g) Not applicable

45. Do you have health insurance?

Yes

No

46. What factors make the care of your child's SCD at home difficult for you?

47. Please add any comments about caring or living with SCD at home 


\section{Appendix 15. Adult Questionaire}

Questionnaire ID..........................................

Clinic Name.

Date.

Place of Residence

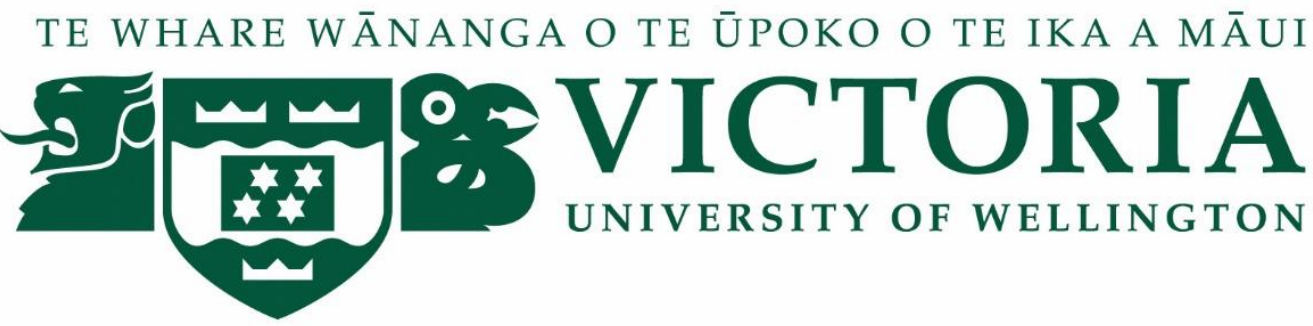

SELF MANAGEMENT STRATEGIES FOR SICKLE CELL DISEASE IN GHANA

Dear respondent. Thank you for agreeing to participate in this survey. This study is intended to investigate what people do for themselves to manage SCD at home and communities. The survey is organised into eight sections (background information; preventive health and maintenance actions; self-monitoring of disease; self-diagnosis; selftreatment; self-management resources; vulnerability and contextual factors; health outcomes. The survey takes approximately 30 minutes to complete. All responses to the survey will be reported in aggregate format. While quotations will be used in the reporting of survey findings, every effort will be made to present these in a non-identifiable way. Consent is assumed by completion and return of the survey. There is no need to sign the survey or identify yourself by name in the survey.

48. What is your age in years?

49. Are you: Male

Female

50. What is the highest year of schooling you completed? (Please circle one)

g) None

h) Primary

i) Junior High/ Middle School

j) Senior High/Secondary

k) Tertiary 
1) Others (please specify)

51. What type of Sickle cell disease do you have?(circle one)

g) $\mathrm{HbSS}$

h) $\mathrm{HbSC}$

i) $\mathrm{Hb}$ SBetha plus thalassaemia

j) $\mathrm{Hb} \mathrm{S}$ Betha zero thalacaemia

k) Don't Know

1) Other (Specify)

52. How old were you when you were first diagnosed with sickle cell disease?

(Years / months / weeks)

\section{PREVENTIVE HEALTH AND MAINTENANCE CARE}

This section asks about things you do or precautions you take to stay healthy and reduce illnesses.

53. How often do you normally do the following to keep well or prevent illness? Circle one number for each item to show how often you have done the activity.

t) Take folic acid Not at all

Daily

Weekly

u) Take vitamin supplements

v) Takes blood tonics

w) Takes malaria prophylaxis

x) Take worm medication

y) Take herbs or traditional

z) Drink water/fluids

aa) Follow a special diet

bb) Avoid strenuous activities

cc) Dress to stay warm

dd) Avoid cold drinks/water

ee) Avoid cold baths
0

$0 \quad 1$

0

0

0

0

0

0

0

0

0

0

0

1

2

2

2

2

2

2

\section{2}

2

2

2

2

2
Monthly

3

3

3

3

3

3

3

3

2

3

3

3 2monthly

4

4

4

4

4

4

4

4

4

4

4

4

4 3months or more 5

5

5

5

5

5

5

5

5

5

5

5
When necessary

6

6

6

6

6

6

6

6

6

6

6

6 


\begin{tabular}{|c|c|c|c|c|c|c|}
\hline ff) Avoid cold places & 0 & 1 & 2 & 3 & 4 & 5 \\
\hline gg) Sleeps in mosquito net & 0 & 1 & 2 & 3 & 4 & 5 \\
\hline hh) Use mosquitoes spray/coil & 0 & 1 & 2 & 3 & 4 & 5 \\
\hline ii) Avoid thinking about disease & 0 & 1 & 2 & 3 & 4 & 5 \\
\hline jj) Attend clinical appointments & 0 & 1 & 2 & 3 & 4 & 5 \\
\hline
\end{tabular}

kk) Other (Specify

\section{SELF MONITORING}

In this section, I will like to find out what you normally do on a daily basis to check the progress of your health

54. Some people say they check their health every day. For example some people inspect their eyes for paleness each day. What do you usually do on a daily basis?

( Circle YES or NO to as many as applied)

1) Check urine colour $\quad$ Yes No

m) Check for jaundice (yellow eyes and body) Yes No

n) Look for palenes $\quad$ Yes No

o) Check temperature $\quad$ Yes No

p) Palpate the abdomen $\quad$ Yes No

q) Look for signs of diarrhoea and vomiting $\quad$ Yes No

r) Listens to body $\quad$ Yes No

s) Keeps a diary or records $\quad$ Yes No

t) Others (please specify) Yes No 


\section{SELF DIAGNOSIS}

In this section, I will like to find out how you usually know that you have a crisis or problems from $S C D$.

55. How many times did you have a painful crisis in the past 12 months?

56. What were the things that causes you to get sick or have painful crisis?

57. How do you usually know that you have a painful crisis?

58. Apart from the experience of painful events, which of the following signs and symptoms do you get when you have sickle cell related problem? ( Circle YES or NO to all that applied)

o) Urine colour changes

Yes No

p) Yellow eyes and body

Yes No

q) High hotness of the body

Yes No

r) Dizziness/ collapse

Yes No

s) Diarrhoea

Yes

No

t) Vomiting

Yes

No

u) Paleness of the eyes and body

Yes

No

v) Swollen joints

Yes

No

w) Abdominal pains

Yes

No

x) Difficulty in breathing

Yes

No

y) Painful erection of the penis (males)

Yes

No

z) Other (please specify)

Yes

No 


\section{SELF TREATMENT}

In this section, I will like to know what you do to care for yourself when you have problems or illness from $S C D$

59. Have you experienced painful episode or crisis in the past 12 months? ( Yes / No)

12a. If YES when was the last time you experienced painful crisis?

(If No go to $Q 13)$

12b. What did you do for yourself to manage the pain crisis?

12c. Please circle how helpful you found what you did for yourself to manage the painful crisis

13a. If $\mathbf{Y E S}$ when was the last time you experienced fever?

(If No go to

Q14)

13b. What did you do for yourself to manage the fever?

13c. Please circle how helpful you found what you did for yourself to manage the fever
a) Very helpful
(ebua me paa)
b) Somehow helpful
(ebua me kakra)
c) Neither helpful nor unhelpful (eho ne ho)
d) Not so helpful
(amoa me papa)
e) Not helpful at all
(amoa me kuraa)
f) Not applicable

60. Have you experienced severe headache in the past 12 months?

(Yes / No) 
14a. If $\mathbf{Y E S}$ when was the last time you experienced headache? Q11)

14b. What did you do for yourself to manage the headache?

14c. Please circle how helpful you found what you did for yourself to manage the severe headache
a) Very helpful
(ebua me paa)
b) Somehow helpful
(ebua me kakra)
c) Neither helpful nor unhelpful (eho ne ho)
d) Not so helpful (amoa me papa)
e) Not helpful at all
(amoa me kuraa)
f) Not applicable

61. Have you experienced sudden weakness or loss of feeling (titiritii) and movement in the past 12 months?

No)

(Yes I

15a. If YES when was the last time you experienced sudden weakness or loss of feeling?

(If NO go Q16)

15b. What did you do for yourself to manage the weakness and loss of feeling?

15c. Please circle how helpful you found what you did for yourself to manage weakness or loss of feeling?
a) Very helpful
(ebua me paa)
b) Somehow helpful
(ebua me kakra)
c) Neither helpful nor unhelpful (eho ne ho)
d) Not so helpful
(amoa me papa)
e) Not helpful at all
(amoa me kuraa)
f) Not applicable

62. Have you experienced leg ulcers (sore /wound) in the past 12months? (Yes / No) 
16a. If YES when was the last time you experienced leg ulcers?

(If No go to

Q17)

16b. What did you do for yourself to manage the leg ulcers?

16c. Please circle how helpful you found what you did for yourself to manage the leg ulcers
a) Very helpful
(ebua me paa)
b) Somehow helpful
(ebua me kakra)
c) Neither helpful nor unhelpful (eho ne ho)
d) Not so helpful
(атоа те рара)
e) Not helpful at all
(amoa me kuraa)
f) Not applicable

63. Have you experienced painful erection of the penis (if male) in the past 12 months

(Yes / No)

17a. If YES when was the last time you experienced painful erection of the penis?....

(If No go to $Q 18$ )

17b. What did you for yourself to manage the painful erection of the penis?

17c. Please circle how helpful you find what you did for yourself to manage the painful erection of the penis
a) Very helpful
(ebua me paa)
b) Somehow helpful
(ebua me kakra)
c) Neither helpful nor unhelpful (eho ne ho)
d) Not so helpful
(amoa me papa)
e) Not helpful at all
(amoa me kuraa)
f) Not applicable

64. Have you experienced yellowing of eyes and body in the past 12 months (Jaundice)

(Yes / No) 
18a. If YES when was the last time you experienced jaundice?

(If No go to

Q16)

18b. What did you do for yourself to manage the jaundice?

18c. Please circle how helpful you found what you did for yourself to manage the jaundice
a) Very helpful
(ebua me paa)
b) Somehow helpful
(ebua me kakra)
c) Neither helpful nor unhelpful (eho ne ho)
d) Not so helpful
(amoa me papa)
e) Not helpful at all
(amoa me kuraa)
f) Not applicable

65. Have you experienced shortage of blood (Paleness of eyes and body) in the past 12 months

(Yes / No)

19a. If YES when was the last time you experienced shortage of blood?

(If No go to 20)

19b. What did you do for yourself to manage the shortage of blood?

19c. Please circle how helpful you found what you did for yourself to manage the shortage of blood
a) Very helpful
(ebua me paa)
b) Somehow helpful
(ebua me kakra)
c) Neither helpful nor unhelpful (eho ne ho)
d) Not so helpful
(amoa me papa)
e) Not helpful at all
(amoa me kuraa)
f) Not applicable 
20a.If YES when was the last time you experienced belly swelling?

(If No go to Q18)

20b. What did you do for yourself to manage the belly swelling?

20c. Please circle how helpful you found what you did for yourself to manage the belly swelling
a) Very helpful
(ebua me paa)
b) Somehow helpful
(ebua me kakra)
c) Neither helpful nor unhelpful (eho ne ho)
d) Not so helpful
(amoa me papa)
e) Not helpful at all
(amoa me kuraa)
f) Not applicable

67. Have you experienced hip problems in the past 12 months

(Yes I

No)

21a. If $\mathbf{Y E S}$ when was the last time you experienced the hip problems?

Q19)

(If No go to

21b. What did you do for yourself to manage the hip problems?

21c. Please circle how helpful you found what you did for yourself to manage the hip problem
a) Very helpful
(ebua me paa)
b) Somehow helpful
(ebua me kakra)
c) Neither helpful nor unhelpful (eho ne ho)
d) Not so helpful
(amoa me papa)
e) Not helpful at all
(amoa me kuraa)
f) Not applicable 
68. Have you experienced sudden seizures or convulsion(fit) in the past 12months (Yes / No)

22a. If YES when was the last time you experienced seizures or convulsion?

(If No go to

22b. What did you do for yourself to manage the seizures or convulsion?

22c. Please circle how helpful you found what you did for yourself to manage the seizures or convulsion
a) Very helpful
(ebua me paa)
b) Somehow helpful
(ebua me kakra)
c) Neither helpful nor unhelpful (eho ne ho)
d) Not so helpful
(amoa me papa)
e) Not helpful at all
(amoa me kuraa)
f) Not applicable

69. Apart from what I have asked you, which other health problem (s) did you have in the past 12 months?

23a. What did you do for yourself to manage it?

23b. How helpful did you find what you did? 
70. How often in the last 12 months did you do the following things to care for yourself when you were sick? (Tick all that applies).

\begin{tabular}{|c|c|c|c|c|c|c|}
\hline & & $\begin{array}{l}\text { Not at } \\
\text { all }\end{array}$ & $\begin{array}{l}\text { Only } \\
\text { once }\end{array}$ & $\begin{array}{c}\text { Some } \\
\text { time }\end{array}$ & $\begin{array}{c}\text { Most of } \\
\text { the } \\
\text { time }\end{array}$ & $\begin{array}{l}\text { All the } \\
\text { time }\end{array}$ \\
\hline s) & Taken pain medication( e.g. Para, Brufen) & 0 & 1 & 2 & 3 & 4 \\
\hline t) & Drunk lot of fluids & 0 & 1 & 2 & 3 & 4 \\
\hline u) & Used traditional medicine & 0 & 1 & 2 & 3 & 4 \\
\hline v) & Applied heat to affected part & 0 & 1 & 2 & 3 & 4 \\
\hline w) & Applied cold to affected part & 0 & 1 & 2 & 3 & 4 \\
\hline x) & Performed wound dressing & 0 & 1 & 2 & 3 & 4 \\
\hline y) & Used massage & 0 & 1 & 2 & 3 & 4 \\
\hline z) & Drunk alcohol & 0 & 1 & 2 & 3 & 4 \\
\hline aa) & Used Indian hemp (wee) & 0 & 1 & 2 & 3 & 4 \\
\hline bb) & Went to bed early & 0 & 1 & 2 & 3 & 4 \\
\hline cc) & Tied affected limb & 0 & 1 & 2 & 3 & 4 \\
\hline dd) & Applied weight to affected limb & 0 & 1 & 3 & 3 & 4 \\
\hline ee) & Said prayers & 0 & 1 & 2 & 3 & 4 \\
\hline ff) & Reduced activities & 0 & 1 & 2 & 3 & 4 \\
\hline gg) & Applied topical cream(e.g. Alcohol) & 0 & 1 & 2 & 3 & 4 \\
\hline hh) & Used exercise or activities & 0 & 1 & 2 & 3 & 4 \\
\hline ii) & Consulted a spiritualist(e.g. Pastor,Mallam) & 0 & 1 & 2 & 3 & 4 \\
\hline jj) & Consulted a doctor/hospital & 0 & 1 & 2 & 3 & 4 \\
\hline
\end{tabular}


71. Have you ever used traditional medicine to manage your SCD?

(Yes / No) (If No go to Q22)

If YES name the medicine, the problem used for and how you used it

\begin{tabular}{|c|c|c|}
\hline Name of medicine & Problems used for & $\begin{array}{l}\text { How used (e.g. } \\
\text { swallowed, applied on } \\
\text { skin, enema) }\end{array}$ \\
\hline \multicolumn{3}{|r|}{ (1) } \\
\hline \multicolumn{3}{|l|}{2.} \\
\hline \multicolumn{3}{|l|}{3.} \\
\hline 4. & & \\
\hline 5. & & \\
\hline 6. & & \\
\hline
\end{tabular}

72. Have you ever used conventional medicines (abrofo duro) on your own to manage your SCD?

(Yes / No)

(If No go to Q23)

If YES name the medicine, the problem used for and how you used it

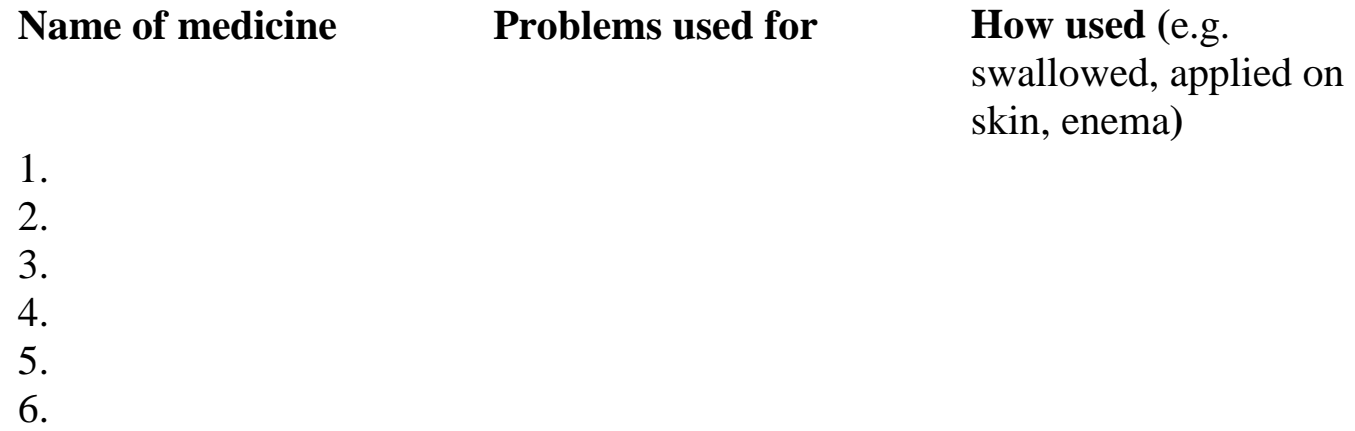

\section{SELF MANAGEMENT RESOURCES}

This section concerns the things you have in the house that you use to manage your SCD, how much you know about caring for SCD at home, and the support you get from other people in managing your SCD.

73. Which of the following resources do you have in the house to help you to manage your SCD? (Please circle YES or No to all the option below)
k) Pain medication (e.g. Para)
Yes
No
1) Folic acid
Yes
No
m) Topical cream
Yes
No 

n) Thermometer
Yes
No
o) Warm clothing
Yes
No
p) Wound dressing equipment
Yes
No
q) Dairy or record book
Yes
No
r) Health education materials eg posters Yes
No
s) Hot water bottle
Yes
No
t) Other (please specify)
Yes
No

74. Tick all sources where you get information on SCD care at home
h) Health workers
Yes
No
i) Relatives
Yes
No
j) Friends
Yes
No
k) Radio
Yes
No
1) Television
Yes
No
m) Internet
Yes
No
n) Other (please specify)
Yes
No

75. Do you attend Sickle Cell Association meetings?

(Yes /

No)

76. How would you rate your knowledge about caring for your SCD at home (Please circle one)
f) Excellent (me nim paa)
g) Very good (me nim kakra)
h) Good (me nim)
i) Fair (3ho ne ho)
j) Poor (me nnim koraa) 
77. Have you ever attended any course, teaching session or read anything about sickle cell care at home? (Yes / No)

( If No go to 33)

78. If YES what was it?

79. Who mainly helps you in caring for your SCD at home? (please circle one)
g) None
h) Parents
i) Relatives
j) Government office e.g. social welfare
k) NGO e.g. sickle cell association
1) Other (Specify).

80. How would you rate the level of support you get for the care of your SCD at home
a) Excellent
(3y3 papaapa)
b) Very good (3y3 paa)
c) Good
$(3 \mathrm{y} 3)$
d) Fair
(3y3 kakra)
e) Poor
(3ny3 koraa)

\section{HEALH OUTCOMES}

In this section, I will like to find out how you view your state of health and your quality of life

81. In general how would you say your health is (wo apom den ete sen)?

(Please circle one)
f) Excellent
(3y3 papaapa)
g) Very good
(3y3 paa)
h) Good
(3y3)
i) Fair
(3y3 kakra)
j) Poor
(3ny3 koraa)

82. How would you rate your overall quality of life? (ase tena mu ete sen ma wo ).Please circle the number below that describes your quality of life in the past 12 months
1
1
2
10

3

45

6

89 


\section{SOCIODEMOGRAPHIC AND HEALTH SERVICES ISSUES}

In this section I will like to get some background information about you, as well as how access sickle cell clinics and other health services to manage your SCD

83. What is your occupation? (Please circle one)
k) Artisan
1) Business/Trading
m) Government employer
n) Private salary worker
o) Retired
p) Sick leave
q) Unemployed
r) Parent
s) Student
t) Other ( please specify)

84. What is your marital status? (Please circle one)
f) Single
g) Married
h) Divorced
i) Widowed
j) In relationship

85. Do you attend sickle cell clinic?

(Yes / No) $\quad($ If No go to 41)

86. If YES to question 40, how many times have you attended the clinic in the past 12 months?.

87. What other services health services do you attend for your SCD care? (E.g. mainstream hospitals, herbal clinic, homeopathic clinic etc.)

88. Approximately how many hours/minutes does it take you to travel from your home to the clinic you attend?
g) Less than 30 minutes
h) 30-60minutes
i) 1-3 hours
j) 4-7 hours 

k) More than 7 hours
1) Not applicable

89. Approximately how much income do you earn in a year in Ghana cedis in a month? (Go to $Q 36$ if you don't receive any income)
i) Less than 100
j) $100-499$
k) $500-899$
l) $900-1299$
m) $1300-1699$
n) $1700-2000$
o) More than 2000
p) None

90. What proportion of your income do you usually spend monthly on managing your sickle cell disease?
h) Just a little
i) Almost half
j) More than half
k) Almost all
1) All of my income
m) Don't know
n) Not applicable

91. Do you have health insurance?

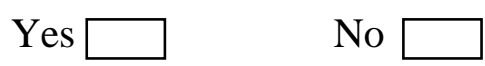

92. What factors make the care of sickle cell disease at home difficult for you?

Please add any comments about caring or living with sickle cell disease at home 


\section{References}

1. Modell, B. and M. Darlison, Global epidemiology of haemoglobin disorders and derived service indicators. Bulletin of the World Health Organization, 2008. 86(6): p. 480-487.

2. UN Recognition of sickle-cell anaemia as a public health problem: $A / R E S / 63 / 237$. Resolution adopted at the general assembly. Sixty-third session. 2009. 1-2.

3. WHO Sickle-cell anaemia: Report by the secretariat (EB 117/34). 2005.

4. MOH Ghana, Strategic framework for the management, prevention and control of sickle cell disease in Ghana, 2011-2015. 2011, Author: Accra Ghana.

5. Ballas, S.K., et al., Beyond the definitions of the phenotypic complications of sickle cell disease: An update on management. Scientific World Journal, 2012. 2012: p. 949535.

6. Myrvik, M.P., et al., Clinically meaningful measurement of pain in children with sickle cell disease. Pediatric Blood \& Cancer, 2013. 60(10): p. 1689-1695.

7. Barr, V.J., et al., The expanded chronic care model: An integration of concepts and strategies from population health promotion and the chronic care model. Hospital Quarterly, 2003. 7: p. 73-81.

8. Conn, V.S., Helping patients help themselves: Chronic disease self-management interventions. Western Journal of Nursing Research, 2011: p. 159-60.

9. Redman, K.B., Patient self-management of chronic disease: The health care providers challenge. 2004, Sadbury: Jones and Bartlet Publishers.

10. Lorig, K. and H. Holmes, Self-management education: History, definition, outcomes, and mechanisms. Ann Behav Med, 2003. 26(1): p. 1-7.

11. McGowan, P. Health promotion, chronic disease, self managment strategies for nurses. 2011 [cited 2014 10/01/14]; Available from: https://www.youtube.com/watch?v=8-CCf8KUZtY.

12. Nuovo, J., Chronic disease management ed. SpringerLink. 2007, New York: Springer-Verlag

13. Department of Health UK Supporting people with long term condition to self care: A guide to developing local strategies and good practice. 2006.

14. Jordan, J.E. and R.H. Osborne, Chronic disease self-management education programs: Challenges ahead. Med J Aust, 2007. 186(2): p. 84-7.

15. Taylor, S.G. and K. Renpenning, Self-care science, nursing theory and evidence-based practice. 2011, New York,USA: Springer Publishing Company, LLC.

16. Chakravorty, S. and T.N. Williams, Sickle cell disease: A neglected chronic disease of increasing global health importance. Arch Dis Child, 2015. 100(1): p. 48-53.

17. Jenerette, C.M., C. Brewer, and A.N. Leak, Self-care recommendations of middle-aged and older adults with sickle cell disease. Nursing Research and Practice, 2011: p. 270594. 
18. McGowan, P., Self managment: A background paper to new perspectives, in International conference on patients self managment. 2005: Victoria, BC.

19. Ryan, P. and K.J. Sawin, The individual and family self-management theory: Background and perspectives on context, process, and outcomes. Nursing Outlook,, 2009. 57(4): p. 217-225. e6.

20. Nakagawa-Kogan, H., et al., Self-management of hypertension: Predictors of success in diastolic blood pressure reduction. Research in Nursing \& Health, 1988. 11(2): p. 105-115.

21. Alderson, M., et al., The program for rheumatic independent self-management: A pilot evaluation. Clinical Rheumatology, 1999. 18(4): p. 283-292.

22. Richard, A.A. and K. Shea, Delineation of Self-Care and Associated Concepts. Journal of Nursing Scholarship, 2011. 43(3): p. 255-264.

23. Clark, M.N., et al., Self management of chronic disease by older adults: $A$ review and questions for research. Journal of Ageing and Health, 1991. 3(3): p. 1-22.

24. Barlow, J., et al., Self-management approaches for people with chronic conditions: A review. Patient Education and Counseling, 2002. 48(2): p. 177-187.

25. Adams, K., A.C. Greiner, and J.M. Corrigan, eds. Report of a summit. The 1st annual crossing the quality chasm summit-A focus on communities. ed. 2004: Washington, DC: National Academies Press.

26. Schulman-Green, D., et al., Processes of self-management in chronic illness. Journal of Nursing Scholarship, 2012. 44(2): p. 136-144.

27. Corbin, J. and A. Strauss, Managing chronic illness at home: Three lines of work. Qualitative Sociology, 1985. 8(3): p. 224-247.

28. Lorig, K., Self-management of chronic illness: A model for the future. Generations, 1993. 17(3): p. 11-14.

29. MOH NZ, Self-management support for people with long-term conditions. 2014, Author: Wellington, New Zealand.

30. Savage, E., et al. Self-management education for cystic fibrosis. Cochrane Database of Systematic Reviews, 2011. DOI: 10.1002/14651858.CD007641.pub2.

31. Quimby, K.R. and I. Hambleton, R., Interventions for caregivers for the recognition of diseaserelated complications in children with sickle cell disease. Cochrane Database of Systematic Reviews, 2013(1): p. 1-8.

32. Grey, M., K. Knafl, and R. McCorkle, A framework for the study of self-and family management of chronic conditions. Nursing Outlook, 2006. 54(5): p. 278-286.

33. Fua, D., et al., Qualitative evaluation of chronic disease self management Program (CDSMP) in Shanghai. Patient Education and Counselling, 2006. 61: p. 389-396. 
34. Kennedy, A., et al., The effectiveness and cost effectiveness of a national lay-led self care support programme for patients with long-term conditions: A pragmatic randomised controlled trial. J Epidemiol Community Health, 2007. 61: p. 254-261.

35. Lorig, K., P.D. Mazonson, and H.R. Holman, Evidence suggesting that health education for selfmanagement in patients with chronic arthritis has sustained health benefits while reducing health care costs. Arthritis and Rheumatism, 1993. 36(4): p. 439-46.

36. Lorig, K., et al., Evidence suggesting that a chronic disease self-management program can improve health status while reducing utilization and costs: A randomized trial. Medical Care, 1999. 37(1): p. 5-14, .

37. Prabhakar, H. Developing an expert patient program for sickle cell disease in the United States: Lessons from the United States and the United Kingdom. 2009.

38. Ghana Statistical Service, Ghana Health Service, and ICF International, Ghana demographic and health survey, G. GSS, ICF International, Editor. 2014, Rockville: Maryland, USA.

39. Ghana Statistical Service, 2010 population and housing census: Provisional results. 2012, Author: Accra, Ghana.

40. Ghanaweb. Ethnic groups. n.d.; Available from:

http://www.ghanaweb.com/GhanaHomePage/tribes/.

41. Heaton, T.B. and A. Darkwah, Religious differences in modernization of the family: Family demographics trends in Ghana. Journal of Family Issues, 2011. 32(12): p. 1576-1596.

42. Komfo Anokye Teaching Hospital. 2015; Available from: http://www.kathhsp.org/aboutus1.php.

43. MOH Ghana, National e-health strategy. 2011: Accra, Ghana.

44. MOH Ghana, WHO pharmaceutical assessment -Level II: Health facility survey in Ghana. 2009, Author: Accra, Ghana.

45. Ghana Health Service, The health sector in Ghana: Facts and figures, P. Division, Editor. 2009, Author: Accra, Ghana.

46. MOH Ghana, National health policy: Creating wealth through health. 2007, Author: Accra, Ghana.

47. MOH Ghana, Policy guidelines on traditional medicine development. 2005, Author: Accra, Ghana.

48. World Health Organisation. World health rankings: Live longer lives better. 2014; Available from: http://www.worldlifeexpectancy.com/country-health-profile/ghana.

49. Population Reference Bereau, 2015 world population data sheet: With a special focus on women's empowerment. 2015, Author.

50. Konotey-Ahulu, F. Sickle cell and other haemoglobinopathies: The genetics that touches you and me. sicklecell.md, 2014. 
51. Edington, G.M., Sickle-Cell Anaemia in the Accra District of the Gold Coast - A review of 20 Cases. British Medical Journal, 1953. 2(4843): p. 957-961.

52. Konotey-Ahulu, F. and B. Ringelhann, Sickle cell anaemia, sickle cell thalassaemia, sickle cell haemoglobin C disease, and asymptomatic haemoglobin C thalassaemia in one Ghanaian family. British Medical Journal, 1969. 1(5644): p. 607-12.

53. Global Newsreel About 18,000 babies born annually with sickle cell disease In Ghana. Modern Ghana, 2013.

54. Dennis-Antwi, J.A., S. Dyson, and K. Ohene-Frempong, Health care provision for sickle cell disease in Ghana: Challenges for the African context. Diversity in Healt and Social Care, 2008. 5 : p. 241-54.

55. Ohene-Frempong, K., et al., Screening newborns for sickle cell disease in Ghana. Paediatrics, 2008. 121: p. S120-S121.

56. CDC. Sickle cell disease (SCD): Data and statistics. [Web page] 2015 February 29/2016 [cited 2015; Available from: http://www.cdc.gov/ncbddd/sicklecell/data.html.

57. Ghana Aids Commission, Summary of the 2013 sentinel survey report. 2013.

58. Ankrah-Badu, G., Epidemiology of sickle cell disease in Ghana, in Symposium on sickle cell disease in Ghana. 2007: Kofi Annan Peace Keeping Centre, Accra, Ghana.

59. Ohene-Frempong, K., National Sickle Cell Programme : A pre-proposal for sickle cell foundation of Ghana. n.d., Sickle Cell Foundation Foundation: Accra, Ghana.

60. Dennis-Antwi, J.A., The social meaning of a child with sickle cell disease in Ghana: Fathers' reactions and perceptions. 2006.

61. WHO Sickle cell disease in the African Region:Current situation and the way forward ( Fifty- six session), report of the regional director. 2006.

62. Dennis-Antwi, J.A. Sickle cell disease services in Ghana. Accra, Ghana. in Symposium on sickle cell disease 2007. Kofi Annan Peace Keeping Centre.

63. Ghana News Agency. Upsurge of sickle cell disease worrying. 2010; Available from: http://www.ghananewsagency.org/science/upsurge-of-sickle-cell-disease-worrying-11087.

64. Dennis-Antwi, J.A., S.A. Opoku, and B. Osei-Amoh, Survey of educational needs of health workers and consumers in Ghana prior to the institution of newborn screening for sickle cell disease in Kumasi. Health Courier, 1995. 5(4): p. 28-32.

65. Piel, F.B., et al., Global epidemiology of sickle haemoglobin in neonates: A contemporary geostatistical model-based map and population estimates. Lancet, 2013. 381(9861): p. 142-151.

66. WHO, Global Epidemiology of haemoglobinopathies. In: Ohene-Frempong (2010). A paper presented at the first global conference on sickle cell disease in Ghana. 2009.

67. Jakubik, L. and M. Thompson, Care of the child with sickle cell disease: Acute complications. Pediatric Nursing, 2000. 26(4). 
68. Gill, V., J. Lavin, and M. Sim, Managing sickle cell disease. Nursing Made Incredibly Easy, 2010. 8(6): p. 24-33.

69. Brown, M., Managing the acutely ill adult with sickle cell disease. Br J Nurs, 2012. 21(2): p. 90-2, 95-6.

70. National Heart Lung \& Blood Institute, Evidence-based management of sickle cell disease. Expert Panel Report, 2014. Bethesda (MD): National Heart, Lung, and Blood Institute, National Institutes of Health, 2014.

71. Herrick, J.B., Peculiar elongated and sickle-shaped red blood corpuscles in a case of severe anemia. 1910. Yale J Biol Med, 2001. 74(3): p. 179-84.

72. Serjeant, G.R., The natural history of sickle cell disease. Cold Spring Harb Perspect Med, 2013. 3(10): p. a011783.

73. WHO Sickle cell disease: A strategy for the WHO African Region (Sixteenth session), report of the regional director. 2010.

74. WHO, Ghana: Health statistics profile $20102010 \mathrm{~b}$.

75. Sickle Cell Foundation of Ghana, National newborn screening for sickle cell disease: Annual report. 2011, Author: Accra, Ghana.

76. Ballas, S.K., More definitions in sickle cell disease: Steady state v base line data. Am J Hematol, 2012. 87(3): p. 338.

77. Rees, D.C., T.N. Williams, and M.T. Gladwin, Sickle-cell disease. Lancet, 2010. 376(9757): p. 201831.

78. Bennett, L., Understanding sickle cell disorders. Nursing Standard, 2005. 19(32): p. 52-61.

79. National Heart Lung \& Blood Institute, The management of sickle cell disease. 2002, USA: NIH Publications.

80. Behrens, R.J. and T.C. Cymet, Sickle cell disorder: Evaluation, treatment, and natural history. Hospital Physician, 2000: p. 17-28.

81. CDC, Sickle cell disease: Current activities, public health implications, and future directions. Journal of Womens' Health, 2007. 16(5).

82. Rees, D.C., The rationale for using hydroxycarbamide in the treatment of sickle cell disease. Haematologica, 2011. 96(4): p. 488-91.

83. Buchanan, G.R., et al., Minor elective surgical procedures under general antesthesia in children with sickle cell anemia without preoperative blood transfusions. Blood, 2003. 102(11): p. 759a759a.

84. Hemker, B.G., et al., When children with sickle-cell disease become adults: Lack of outpatient care leads to increased use of the emergency department. Am J Hematol, 2011. 86(10): p. 863-5. 
85. Jones, S., et al., Windy weather and low humidity are associated with an increased number of hospital admissions for acute pain and sickle cell disease in an urban environment with a maritime temperate climate. Br J Haematol, 2005. 131(4): p. 530-3.

86. Stuart, M.J. and R.L. Nagel, Sickle-cell disease. Lancet, 2004. 364(9442): p. 1343-1360.

87. WHO Prevalance of Sickle Cell Anaemia: Report by the Secretariat (A59/9). 2006.

88. Yanni, E., et al., Trends in pediatric sickle cell disease-related mortality in the United States, 1983-2002. J Pediatr, 2009. 154(4): p. 541-5.

89. Platt, O.S., et al., Mortality in sickle cell disease -- Life expectancy and risk factors for early death. The New England Journal of Medicine, 1994. 330(23): p. 1639-1644.

90. Wierenga, K.J., I.R. Hambleton, and N.A. Lewis, Survival estimates for patients with homozygous sickle-cell disease in Jamaica: A clinic-based population study. Lancet, 2001. 357(9257): p. 680-3.

91. Adegoke, S.A. and E.A. Kuteyi, Psychosocial burden of sickle cell disease on the family, Nigeria. African Journal of Primary Health Care \& Family Medicine, 2012. 4(1): p. 6.

92. Anie, K.A., F.E. Egunjobi, and O.O. Akinyanju, Psychosocial impact of sickle cell disorder: Perspectives from a Nigerian setting. Global Health, 2010. 6: p. 2.

93. Wonkam, A., et al., Psychosocial stressors of sickle cell disease on adult patients in Cameroon. J Genet Couns, 2014. 23(6): p. 948-56.

94. Dennis-Antwi, J.A., et al., 'I can die today, I can die tomorrow': Lay perceptions of sickle cell disease in Kumasi, Ghana at a point of transition. Ethnicity \& Health, 2011. 16(4-5): p. 465-481.

95. Khattah, A., B. Rawlings, and I. Ali, Care of patients with haemoglobin abnormaiities: Nursing management. British Journal of Nursing, 2006. 15(19).

96. Brawley, O.W., et al., National institutes of health consensus development conference statement: Hydroxyurea treatment for sickle cell disease. Annals of Internal Medicine, 2008. 148(12): p. 932-+.

97. Bernaudin, F. and M. Kuentz, Haplo-BMT: cure or back to sickle cell? Blood, 2012. 120(22): p. 4276-7.

98. Smith, W.R. and A.A. Thompson, Indications and complications of transfusions in sickle cell disease. Pediatr Blood Cancer, 2012. 59(2): p. 358-64.

99. Alvarez, O., et al., Effect of hydroxyurea treatment on renal function parameters: Results from the multi-center placebo-controlled BABY HUG clinical trial for infants with sickle cell anemia. Pediatr Blood Cancer, 2012. 59(4): p. 668-74.

100. National Heart Lung \& Blood Institute, Evidenced-based management of sickle cell disease; Expert panel report, US Department of Health and Human Services: National Institute of Health, Editor. 2014.

101. Edwards, L.Y. and C.L. Edwards, Psychosocial treatments in pain management of sickle cell disease. J Natl Med Assoc, 2010. 102(11): p. 1084-94. 
102. Okpala, I., et al., The comprehensive care of sickle cell disease. Eur J Haematol, 2002. 64.

103. Gill, V., J. Lavin, and M. Sim Managing sickle cell disease. 2010.

104. Tangayi, S., Framework for thalacaemia and sickle cell disease. Nursing Times, 2011. 107(41): p. 12-13.

105. International Association of Sickle Cell Nursing. n.d.; Available from: http://www.iascnapa.org/.

106. Mahat, G., M.A. Scoloveno, and C.B. Donnelly, Written educational materials for families of chronically ill children. J Am Acad Nurse Pract, 2007. 19(9): p. 471-476.

107. Jennifer, M., et al., The possible impact of teachers and school nurses on the lives of children living with sickle cell disease. Journal of School Health, 2011. 81(5).

108. Khattab, A.D., B. Rawlings, and I.S. Ali, Care of patients with haemoglobin abnormalities: History and biology. British Journal of Nursing, 2006. 15(18): p. 994-998.

109. MOH Ghana, Under 5 Child Health Strategy: 2007-2015. 2009, Author: Accra, Ghana.

110. Ghana Health Service, Creating wealth through health. The health sector programme of work: 2007-2011. 2008, Author: Accra, Ghana

111. Ohene-Frempong, K., Update of sickle cell programming in Ghana, A.A. Druye, Editor. 2016.

112. Druye, A.A., Education and counselling for patients and families with SCD in Ghana, in Symposium on sickle cell disease 2007: Kofi Annan Peace Keeping Centre, Accra, Ghana.

113. Business Day Ghana, Ghana: GNPC donates U.S.\$4.5 million for construction of sickle cell centre. 2016.

114. MOH Ghana, Standard treatment guidelines, Author, Editor. 2010, Author: Accra, Ghana.

115. Petersen, A., The best experts: The narratives of those who have a genetic condition. Social Science \& Medicine, 2006. 63(1): p. 32-42.

116. Dampier, C., et al., Physical and cognitive-behavioral activities used in the home management of sickle pain: A daily diary study in children and adolescents. Paediatric Blood \& Cancer, 2004. 43(6): p. 674-678.

117. Howard, J., et al., Cannabis use in sickle cell disease: A questionnaire study. Br J Haematol, 2005. 131(1): p. 123-8.

118. Jenerette, C.M. and G. Lauderdale, Successful aging with sickle cell disease: Using qualitative methods to inform theory. Journal of Theory Construction \& Testing, 2008. 12(1): p. 16-24.

119. Levenson, J.L., et al., Alcohol abuse in sickle cell disease: The PISCES project. American Journal on Addictions, 2007. 16(5): p. 383-388.

120. Sangho, H., et al., Management of sickle cells disease by households in Bamako. Le Mali Medical, 2009. 24(3): p. 53-6. 
121. Sibinga, E.M.S., et al., Pediatric patients with sickle cell disease: Use of complementary and alternative therapies. Journal of Alternative \& Complementary Medicine, 2006. 12(3): p. 291298.

122. Smith, W.R., et al., Daily assessment of pain in adults with sickle cell disease. Ann Intern Med, 2008. 148(2): p. 94-101.

123. Tanabe, P., et al., A qualitative analysis of best self-management practices: sickle cell disease. J Natl Med Assoc, 2010. 102(11): p. 1033-41.

124. Tanase, A. and R. Zanni, The use of complementary and alternative medicine among pediatric cystic fibrosis patients. J Altern Complement Med, 2008. 14(10): p. 1271-3.

125. Yoon, S.L. and S. Black, Comprehensive, integrative management of pain for patients with sicklecell disease. Journal of Alternative \& Complementary Medicine, 2006. 12(10): p. 995-1001.

126. Blau, H., et al., Microbial contamination of nebulizers in the home treatment of cystic fibrosis. Child Care Health and Development, 2007. 33(4): p. 491-5.

127. McGuffie, K., et al., Self-reported involvement of family members in the care of adults with CF. Journal of Cystic Fibrosis, 2008. 7(2): p. 95-101.

128. Stenekes, S.J., et al., Frequency and self-management of pain, dyspnea, and cough in cystic fibrosis. Jounal of Pain and Symptom Management, 2009. 38(6): p. 837-48.

129. Wong, M.G. and S.A. Heriot, Parents of children with cystic fibrosis: How they hope, cope and despair. Child care Health and Development, 2008. 34(3): p. 344-54.

130. Sholapur, N.S., et al., A survey of patients with haemophilia to understand how they track product used at home. Haemophilia, 2013. 19(5): p. e289-e295.

131. Jacob, E., et al., Remote monitoring of pain and symptoms using wireless technology in children and adolescents with sickle cell disease. Journal of the American Association of Nurse Practitioners, 2013. 25(1): p. 42-54.

132. McClellan, C.B., et al., Use of handheld wireless technology for a home-based sickle cell pain management protocol. Journal of Paediatric Psychology, 2009. 34(5): p. 564-573.

133. Arias Llorente, R.P., C. Bousono Garcia, and J.J. Diaz Martin, Treatment compliance in children and adults with cystic fibrosis. Journal of Cystic Fibrosis, 2008. 7(5): p. 359-67.

134. Baker, L.K. and M.J. Denyes, Predictors of self-care in adolescents with cystic fibrosis: A test of Orem's theories of self-care and self-care deficit. Journal of Pediatric Nursing, 2008. 23(1): p. 3748.

135. Dalcin Pde, T., et al., Adherence to treatment in patients with cystic fibrosis. Journal Brasileiro De Pneumologia, 2007. 33(6): p. 663-70.

136. Daniels, T., et al., Accurate assessment of adherence: Self-report and clinician report vs electronic monitoring of nebulizers. Chest, 2011. 140(2): p. 425-32. 
137. Fiese, B.H. and R.S. Everhart, Medical adherence and childhood chronic illness: Family daily management skills and emotional climate as emerging contributors. Current Opinion in Pediatrics, 2006. 18(5): p. 551-7.

138. Walker, I., et al., Comparing hand-held computers and paper diaries for haemophilia home therapy: A randomized trial. Haemophilia, 2004. 10(6): p. 698-704.

139. Lemanek, K.L., M. Ranalli, and C. Lukens, A randomized controlled trial of massage therapy in children with sickle cell disease. J Pediatr Psychol, 2009. 34(10): p. 1091-1096.

140. Cummings, E., et al., Enhancing self-efficacy for self-management in people with cystic fibrosis. Studies in Health Technology and Infomatics, 2011. 169: p. 33-7.

141. Grzincich, G., et al., Evaluation of a home telemonitoring service for adult patients with cystic fibrosis: A pilot study. J Telemed Telecare, 2010. 16(7): p. 359-62.

142. Moorcroft, A.J., et al., Individualised unsupervised exercise training in adults with cystic fibrosis: A 1 year randomised controlled trial. Thorax, 2004. 59(12): p. 1074-80.

143. Leone, J.R., Utility of a wireless, handheld monitoring system in the management of hemophilia patients. Comput Inform Nurs, 2011. 29(9): p. 521-2.

144. Teitel, J., et al., A Canadian survey of self-infusion practices in persons with haemophilia Haemophilia, 2012. 18(6): p. e403-e405.

145. Balaguer, A. and J. Gonzalez de Dios, Home versus hospital intravenous antibiotic therapy for cystic fibrosis. Cochrane Database Syst Rev, 2012. 3: p. Cd001917.

146. Watson, H., D. Bilton, and H. Truby, A randomized controlled trial of a new behavioral homebased nutrition education program, "Eat Well with CF," in adults with cystic fibrosis. J Am Diet Assoc, 2008. 108(5): p. 847-52.

147. Johnson, L., Sickle cell disease patients and patient-controlled analgesia. British Journal of Nursing, 2003. 12(3): p. 144-153.

148. George, M., et al., Perceptions of barriers and facilitators: Self-management decisions by older adolescents and adults with CF. J Cyst Fibros, 2010. 9(6): p. 425-32.

149. Happ, M.B., et al., Parent and child perceptions of a self-regulated, home-based exercise program for children with cystic fibrosis. Nursing Research, 2013. 62(5): p. 305-314.

150. Hayes, C.C. and E. Savage, Fathers' perspectives on the emotional impact of managing the care of their children with cystic fibrosis. Journal of Paediatric Nursing, 2008. 23(4): p. 250-6.

151. Lane, S., et al., What should men living with severe haemophilia need to know? The perspectives of Canadian haemophilia health care providers. Haemophilia, 2013. 19(4): p. 503-510.

152. Llewellyn, C.D., et al., The illness perceptions and treatment beliefs of individuals with severe haemophilia and their role in adherence to home treatment. Psychology \& Health, 2003. 18(2): p. $185-200$. 
153. Mondorf, W., et al., Haemoassist ${ }^{\text {TM }}$ - a hand-held electronic patient diary for haemophilia home care. Haemophilia, 2009. 15(2): p. 464-472.

154. Aisiku, I.P., et al., Comparisons of high versus low emergency department utilizers in sickle cell disease. Annals of Emergency Medicine, 2009. 53(5): p. 587-593.

155. Jenerette, C.M. and C. Murdaugh, Testing the theory of self-care management for sickle cell disease. Research in Nursing \& Health, 2008. 31(4): p. 355-369.

156. Jenerette, C.M. and R.C.S. Phillips, An examination of differences in intra-personal resources, self-care management, and health outcomes in older and younger adults with sickle cell disease. Southern Online Journal of Nursing Research, 2006. 7(3): p. 1-24.

157. Grossoehme, D.H., R. Vandyke, and M. Seid, Spirituality's role in chronic disease selfmanagement: Sanctification of the body in families dealing with cystic fibrosis. Journal of Health Care Chaplaincy, 2008. 15(2): p. 149-158.

158. Moen, A., et al., Enhancing self-efficacy for self-management in people with cystic fibrosis. Studies in Health Technology \& Informatics, 2011. 169: p. 33-37.

159. Schrijvers, L.H., et al., Learning intravenous infusion in haemophilia: Experience from the Netherlands. Haemophilia, 2012. 18(4): p. 516-520.

160. Elander, J. and T. Barry, Analgesic use and pain coping among patients with haemophilia. Haemophilia, 2003. 9(2): p. 202-213.

161. Criner, G.J., Ambulatory home oxygen: What is the evidence for benefit, and who does it help? Respiratory Care, 2013. 58(1): p. 48-64.

162. Howard, J., Managing acute painful sickle cell episodes in hospital. Nurs Times, 2012. 108(39): p. 23.

163. Khair, K., M. Holland, and S. Carrington, Social networking for adolescents with severe haemophilia. Haemophilia, 2012. 18(3): p. e290-e296.

164. Negrier, C., et al., The benefits of exercise for patients with haemophilia and recommendations for safe and effective physical activity. Haemophilia, 2013. 19(4): p. 487-498.

165. Hill, K., et al., Effectiveness of a balance training home exercise programme for adults with haemophilia: A pilot study. Haemophilia, 2010. 16(1): p. 162-9.

166. Grossoehme, D.H., et al., Is adolescents' religious coping with cystic fibrosis associated with the rate of decline in pulmonary function?-A preliminary study. J Health Care Chaplain, 2013. 19(1): p. 33-42.

167. Wiedebusch, S., et al., Quality of life, psychosocial strains and coping in parents of children with haemophilia. Haemophilia, 2008. 14(5): p. 1014-1022.

168. Atkins, R.C. and M.C. Walters, Haematopoietic cell transplantation in the treatment of sickle cell disease. Expert Opinion on Biological Therapy, 2003. 3(8): p. 1215-1224. 
169. Dashiff, C., et al., Cystic fibrosis-related diabetes in older adolescents: Parental support and selfmanagement. J Spec Pediatr Nurs, 2013. 18(1): p. 42-53.

170. Canclini, M., et al., Psychological aspects and coping in haemophilic patients: A case-control study. Haemophilia, 2003. 9(5): p. 619.

171. While, A.E. and J. Mullen, Living with sickle cell disease: The perspective of young people. British Journal of Nursing, 2004. 13(6).

172. Matthie, N., C. Jenerette, and S. McMillan, Role of self-care in sickle cell disease. Pain Management Nursing, 2015. 16(3): p. 257-266.

173. Forrester, A.B., et al., The lived experiences of adolescents with sickle cell disease in Kingston, Jamaica. Int J Qual Stud Health Well-being, 2015. 10: p. 28104.

174. Ferreira, S.L., et al., Vulnerability in adults with sickle cell disease: Subsidies for nursing care. Ciencia, Cuidado e Saude, 2013. 12(4): p. 711-718 8p.

175. Dorsey, C.J. and C.L. Murdaugh, The theory of self-care management for vulnerable populations. Journal of Theory Construction \& Testing, 2003. 7(2): p. 43-49.

176. Ballas, S.K., Self-management of sickle cell disease: A new frontier. J Natl Med Assoc, 2010. 102(11): p. 1042-3.

177. Cordeiro, R.C., S.L. Ferreira, and A.C. Santos, Experiences of illness among individuals with sickle cell anemia and self-care strategies. Acta Paulista de Enfermagem, 2014. 27(6): p. 499-504.

178. Maxwell, J., Conceptual framework: What do you think is going on. 2005). Qualitative research design: An interactive approach, 2005: p. 33-63.

179. Kirk, S., et al., The effectiveness of self-care support interventions for children and young people with long-term conditions: A systematic review. Child: Care, Health \& Development, 2013. 39(3): p. 305-324.

180. McBain, H., M. Shipley, and S. Newman, The impact of self-monitoring in chronic illness on healthcare utilisation: A systematic review of reviews. BMC Health Services Research, 2015. 15(1): p. 1.

181. McManus, R.J., et al., Effect of self-monitoring and medication self-titration on systolic blood pressure in hypertensive patients at high risk of cardiovascular disease: The TASMIN-SR randomized clinical trial. JAMA, 2014. 312(8): p. 799-808.

182. Franciosi, J., et al., Hypoxia-induced acute lung injury in murine models of sickle cell disease. Free Radical Biology and Medicine, 2002. 33: p. S366-S366.

183. Chidum, E., et al., Self-monitoring of blood glucose improved glycaemic control and 10-year coronary heart disease risk profile of type 2 diabetic patients. Chinese Medical Journal-Beijing, 2011. 124(2): p. 166.

184. Eastwood, C.A., et al., Weight and symptom diary for self-monitoring in heart failure clinic patients. Journal of Cardiovascular Nursing, 2007. 22(5): p. 382-389. 
185. Bandura, A., Social cognitive theory of self-regulation. Organizational Behavior and Human Decision Processes, 1991. 50(2): p. 248-287.

186. Creswell, J.W. and C.L. Plano, Designing and conducting mixed methods research. 2011, Califonia: SAGE Publications, Inc.

187. Creswell, J.W., Research design: Qualitative and quantitative and mixed method approach. Kindle ed. 2013: SAGE Publications. .

188. Neuendorf, A., The content analysis quide book. 2002, Chicago: Sage Publication, Inc.

189. Krippendorff, K., Content analysis: An introduction to its methodology. 2013, Thousand Oaks, Calif: SAGE Publications.

190. Hsieh, H.F., Deductive content analysis of end-of-life decision-making in the ICU. 2004.

191. Berg, B., An introduction to content analysis, in In qualitative research methods in social sciences. 2004, Pearson: New York. p. 265-297.

192. Kim, I. and J. Kuljis. Applying content analysis to web based content. in ITI International Conference on Information Technology Interface. 2010. Cavtat: IEEE.

193. Wang, C.J., et al., Quality-of-Care Indicators for Children With Sickle Cell Disease. Pediatrics, 2011. 128(3): p. 484-493.

194. Liang, S.-Y., Y.-H. Chuang, and S.-F. Wu, Preliminarily application of content analysis to qualitative nursing data. Journal of Nursing, 2012. 59(5): p. 84-90.

195. Moonaghi, H.K., M.R. Ahanchian, and Z.M. Hassanian, A qualitative content analysis of knowledge storage in nursing education system. Iranian Red Crescent Medical Journal, 2014. 16(10): p. 1.

196. Sigsby, L.M. and D.W. Campbell, Nursing Interventions Classification: A content analysis of nursing activities in public schools. Journal of Community Health Nursing, 1995. 12(4): p. 229237.

197. Lee, K.-J., et al., Analysis of trends and contents of nursing doctoral dissertations in Korea. Journal of Korean Academy of Nursing 2012. 42(2): p. 302.

198. Loomis, M.E., Emerging content in nursing: An analysis of dissertation abstracts and titles. Nursing Research, 1985. 34(2): p. 113-119.

199. Wagner, L.S., Nursing administration graduate education: A content analysis of selected nursing publications, 1976-1985. 1986, University of Florida

200. Guo, J.-W., et al., Using content analysis to identify study eligibility criteria concepts in cancer nursing research. Computers, Informatics, Nursing 2014. 32(7): p. 333-342.

201. Gebru, K., A. Willman, and T.H. Blekinge, Education to promote culturally competent nursing care - A content analysis of student responses. Nurse Education Today, 2010. 30(1): p. 54-60. 
202. Ferrell, B., et al., Analysis of palliative care content in nursing textbooks. Journal of Palliative Care, 2000. 16(1): p. 39-47.

203. McEwen, M., Analysis of spirituality content in nursing textbooks. Journal of Nursing Education, 2004. 43(1): p. 20-30.

204. Palese, A., et al., Health projects managed by nursing coordinators: An analysis of contents and degree of success. Assistenza Infermieristica e Ricerca : AIR, 2012. 31(4): p. 177-183.

205. Powers, M.J., S.P. Murphy, and P.J. Wooldridge, Validation of two experimental nursing approaches using content analysis. Research in Nursing \& Health, 1983. 6(1): p. 3-9.

206. Barrett, M.E., The image of nursing on television: $A$ content analysis. 1994, ProQuest Dissertation and Thesis database, (UMI No. Z39.88-2004).

207. McMillan, S.J., The microscope and the moving target: The challenge of applying content analysis to the World Wide web. Journalism and Mass Communication Quarterly, 2000. 77(1): p. 80-98.

208. Morgan, D.L., Qualitative content analysis: A guide to paths not taken. Qualitative Health Research, 1993. 3(1): p. 112-21

209. Baskin, M.N., et al., Bacteremia risk and outpatient management of febrile patients with sickle cell disease. Pediatrics, 2013. 131(6): p. 1035-1041.

210. Hsieh, H.F. and S.E. Shannon, Three approaches to qualitative content analysis. Qual Health Res, 2005. 15(9): p. 1277-88.

211. Elo, S. and H. Kyngäs, The qualitative content analysis process. Journal of Advanced Nursing, 2008. 62(1): p. 107-115.

212. Babbie, E.R., The practice of social research. 12th ed. 2010: Belmont, Calif : Wadsworth Cengage.

213. Solomon, L.R., Pain management in adults with sickle cell disease in a medical center emergency department. Journal of the National Medical Association, 2010. 102(11): p. 1025-1032.

214. Trochim, W.M. and J.P. Donnelly. Research methods knowledge base. 2001; Available from: http://www.socialresearchmethods.net/kb/.

215. Graneheim, U.H. and B. Lundman, Qualitative content analysis in nursing research: Concepts, procedures and measures to achieve trustworthiness. Nurse Education Today, 2004. 24(2): p. 105-112.

216. Gillis, A. and W. Jackson, Research for nurses: Methods and interpretations. 2002, Philadelphia: F. A. Davis Company.

217. Hildenbrand, A.K., et al., Psychosocial and pharmacological management of pain in pediatric sickle cell disease. Postgraduate Medicine, 2014. 126(2): p. 123-133.

218. Berthet, S., et al., [Neonatal screening for sickle cell disease at the Nice University Hospital: The last 8 years. Arch Pediatr, 2010. 17(12): p. 1652-6. 
219. Guthrie, G., Basic Research Methods: An entry to social science research. 2010, SAGE Publications India Pvt Ltd.

220. Gibbs, G., Question types \& piloting. Part 1 of 3 on questionnaire design 2012.

221. Stanford School of Medicine. Chronic disease self-management program (Better choices, better health ${ }^{\circledR}$ workshop). 2014; Available from:

http://patienteducation.stanford.edu/programs/cdsmp.html.

222. Creative Research Systems. Sample size calculator. 2012 [cited 2014 22/02/14]; Available from: http://www.surveysystem.com/sscalc.htm.

223. Survey Monkey. Survey sample size. 1999-2014 [cited 2014 22/02/14]; Available from: https://www.surveymonkey.com/mp/sample-size/.

224. Explorable. Non-probability sampling. 2008-2016 [cited 201612 Novembr 2016]; Available from: https://explorable.com/non-probability-sampling.

225. Kent, R.A., Analysing quantitative data : Variable-based and case-based approaches to nonexperimental datasets. 2015, Los Angeles Sage Publications Ltd.

226. Rovai, P.A., J.D. Baker, and K. Ponton, Social science research design and statistics: A guide to research methods and SPSS analysis. 2013, Chesapeake, VA: Watertree Press.

227. Adzika, V.A., F.N. Glozah, and C.S. Ahorlu, Socio-demographic characteristics and psychosocial consequences of sickle cell disease: The case of patients in a public hospital in Ghana. Journal of Health, Population and Nutrition, 2017. 36(1): p. 4.

228. Santos, J.P.d. and M. Gomes Neto, Sociodemographic aspects and quality of life of patients with sickle cell anemia. Revista Brasileira de Hematologia e Hemoterapia, 2013. 35(4): p. 242-245.

229. Grosse, S.D., et al., Sickle cell disease in Africa: A neglected cause of early childhood mortality. American Journal of Preventive Medicine, 2011. 41(6): p. S398-S405.

230. Makani, J., et al., Mortality in sickle cell anemia in Africa: A prospective cohort study in Tanzania. PloS one, 2011. 6(2): p. e14699.

231. Nukunya, G.K., Tradition and change in Ghana: An introduction to sociology. 2003: Ghana Universities Press.

232. Ampim, G.A., Men's involvement in maternal healthcare in Accra, Ghana. From household to dlivery room. 2013, MSc thesis, The University of Bergen.

233. Ghana Statistical Service, Ghana living standards survey round 6: Main report, G.S. Service, Editor. 2014, Author: Accra, Ghana.

234. Citifm. Daily minimum wage increased to GHÇ8. 2015 [cited 2016 15/09/16]; Available from: http://citifmonline.com/2015/10/03/daily-minimum-wage-increased-to-gh\%C2\%A28/.

235. Wilde, M.H. and S. Garvin, A concept analysis of self-monitoring. Journal of Advanced Nursing, 2007. 57(3): p. 339-350. 
236. Mattila, E., et al., Empowering citizens for well-being and chronic disease management with wellness diary. IEEE Transactions on Information Technology in Biomedicine, 2010. 14(2): p. 456463.

237. Tracy, M.F., Recognition of heart failure symptom patterns through facilitated personal awareness. 1999, Doctoral dissertation, University of Minnesota.

238. Jacob, E., et al., Are there phases to the vaso-occlusive painful episode in sickle cell disease? Journal of Pain and Symptom Management, 2005. 29(4): p. 392-400.

239. Alkon, A., et al., Health and safety checklist for early care and education programs to assess key national health and safety standards. Maternal and Child Health Journal, 2016. 20(1): p. 114127.

240. Herbpathy. Turkey berry herb uses, benefits, cures, side effects, nutrients. 2016; Available from: http://herbpathy.com/Uses-and-Benefits-of-Turkey-Berry-Cid3961.

241. Ansah, E.K., et al., The impact of providing rapid diagnostic malaria tests on fever management in the private retail sector in Ghana: A cluster randomized trial. British Medical Journal, 2015. 350: p. h1019.

242. Minstry of Health, Standard treatment guidelines. 2010, Author: Accra, Ghana.

243. Ghana Health Service. National malaria control programme: Programme background. 2015; Available from: http:// www.ghanahealthservice.org/ghs-subcategory. php?cid=4\&scid=41.

244. Gyasi, R.M., L.P. Siaw, and C.M. Mensah, Prevalence and pattern of traditional medical therapy utilisation in Kumasi Metropolis and Sekyere South District, Ghana. J Ethnopharmacol, 2015. 161: p. 138-46.

245. Essegbey, G.O. The dynamics of innovation in traditional medicine in Ghana. WIPA Magazine, 2015.

246. Cotton, S., D. Grossoehme, and M.E. McGrady, Religious coping and the use of prayer in children with sickle cell disease. Pediatric Blood \& Cancer, 2012. 58(2): p. 244-249.

247. Andrade, C., Prayer and healing: A medical and scientific perspective on randomized controlled trials. Indian J Psychiatry, 2009. 51(4): p. 247-253.

248. Grossoehme, D.H., et al., Parents' religious coping styles in the first year after their child's cystic fibrosis diagnosis. J Health Care Chaplain, 2010. 16(3-4): p. 109-22.

249. Hoffer, L.J., Folate supplementation in sickle cell anemia. New England Journal of Medicine, 2003. 349(8): p. 813-813.

250. Dixit, R., et al., Folate supplementation in people with sickle cell disease. The Cochrane Library, 2016. CD011130.(2).

251. Okomo, U. and M.M. Meremikwu, Fluid replacement therapy for acute episodes of pain in people with sickle cell disease. Cochrane Database Syst Rev, 2015. 3: p. Cd005406.

252. Meremikwu, M.M. and U. Okomo, Sickle cell disease. BMJ Clinical Evidence, 2011. 2011. 
253. Redwood, A.M., et al., Climate and painful crisis of sickle-cell disease in Jamaica. British Medical Journal, 1976. 1(6001): p. 66.

254. Mohan, J., et al., Peripheral vascular response to mild indirect cooling in patients with homozygous sickle cell (SS) disease and the frequency of painful crisis. Clinical Science, 1998. 94(2): p. 111-120.

255. Hernandez, L.M., Measures of health literacy workshop summary. 2009, Washington, D.C. : Institute of Medicine of the National Academies : The National Academies Press Washington, D.C.

256. Eric, S.D., et al., Self-medication practices with antibiotics among tertiary level students in Accra, Ghana: A cross-sectional study. International Journal of Environmental Research and Public Health, 2012. 10.

257. Onakpoya, I.J., G. Hayward, and C.J. Heneghan, Antibiotics for preventing lower respiratory tract infections in high -risk children aged 12 years and under. The Cochrane Library, 2015. CD011530.

258. Hirst, C. and S. Owusu-Ofori, Prophylactic antibiotics for preventing pneumococcal infection in children with sickle cell disease. The Cochrane Library, 2012. CD003427.

259. Adjei, E., L. Nunoo, and E. Yankey, Some Ghanaian blood tonics as sources of iron and other trace elements. Journal of Science and Technology, 2009. 29(1): p. 76-84.

260. Patel, R.I. and R.D. Beckett, Evaluation of resources for analyzing drug interactions. Journal of the Medical Library Association: JMLA, 2016. 104(4): p. 290.

261. Oshikoya, K.A., et al., Use of complementary and alternative medicines for children with chronic health conditions in Lagos, Nigeria. BMC Complementary and Alternative Medicine, 2008. 8: p. 66-66.

262. Nsimba, M.M., et al., Effect of a Congolese herbal medicine used in sickle cell anemia on the expression of plasminogen activators in human coronary aortic endothelial cells culture. J Ethnopharmacol, 2013. 146(2): p. 594-9.

263. Ameh, S.J., F.D. Tarfa, and B.U. Ebeshi, Traditional herbal management of sickle cell anemia: Lessons from Nigeria. Anemia, 2012. 2012: p. 607436.

264. Oniyangi, O. and D.H. Cohall, Phytomedicines (medicines derived from plants) for sickle cell disease. Cochrane Database Syst Rev, 2015. 4: p. Cd004448.

265. Cordeiro, N.J. and O. Oniyangi, Phytomedicines (medicines derived from plants) for sickle cell disease. Cochrane Database Syst Rev, 2004(3): p. CD004448.

266. Perampaladas, K., et al., The road to commercialization in Africa: Lessons from developing the sickle-cell drug Niprisan. BMC Int Health Hum Rights, 2010. 10 (1): p. 11.

267. Denisco, R.A., R.K. Chandler, and W.M. Compton, Addressing the intersecting problems of opioid misuse and chronic pain treatment. Experimental and Clinical Psychopharmacology, 2008. 16(5): p. 417. 
268. Kotila, T., et al., Addiction or pseudoaddiction in sickle cell disease patients: Time to decide-a case series. Annals of Ibadan Postgraduate Medicine, 2015. 13(1): p. 44-47.

269. New Crusading Guide. Silent reality : Truths and tales of the sickle cell disorder in Ghana and Nigeria. 2014 Available from:

http://www.peacefmonline.com/pages/local/health/201403/192978.php.

270. Flinders University, Educating future health care professionals to support people with chronic conditions to live better and live longer: A chronic condition self-management support tertiary education curriculum framework. 2007: Adelaide, Australia.

271. Ghana College of Nursing. Specialist education in nursing, midwifery and related disciplines. 2016 20/12/16]; Available from: http://www.gcnm.edu.gh/. 